University of Louisville

ThinkIR: The University of Louisville's Institutional Repository

Electronic Theses and Dissertations

$5-2020$

\title{
The cardiovascular effects of tobacco product-derived aldehydes: contribution of transient receptor potential ankyrin-1.
}

Lexiao Jin

University of Louisville

Follow this and additional works at: https://ir.library.louisville.edu/etd

Part of the Toxicology Commons

\section{Recommended Citation}

Jin, Lexiao, "The cardiovascular effects of tobacco product-derived aldehydes: contribution of transient receptor potential ankyrin-1." (2020). Electronic Theses and Dissertations. Paper 3429.

https://doi.org/10.18297/etd/3429

This Doctoral Dissertation is brought to you for free and open access by ThinkIR: The University of Louisville's Institutional Repository. It has been accepted for inclusion in Electronic Theses and Dissertations by an authorized administrator of ThinkIR: The University of Louisville's Institutional Repository. This title appears here courtesy of the author, who has retained all other copyrights. For more information, please contact thinkir@louisville.edu. 
THE CARDIOVASCULAR EFFECTS OF TOBACCO PRODUCT-DERIVED

ALDEHYDES:

CONTRIBUTION OF TRANSIENT RECEPTOR POTENTIAL ANKYRIN-1

\author{
By \\ Lexiao Jin \\ M.S., Wenzhou Medical University, 2008 \\ M.D., Wenzhou Medical University, 2003

\begin{abstract}
A Dissertation
Submitted to the Faculty of the

School of Medicine of the University of Louisville

In Partial Fulfillment of the Requirements

for the Degree of
\end{abstract} \\ Doctor of Philosophy in Pharmacology and Toxicology \\ Department of Pharmacology and Toxicology \\ University of Louisville \\ Louisville, KY
}

May, 2020 

THE CARDIOVASCULAR EFFECTS OF TOBACCO PRODUCT-DERIVED

ALDEHYDES:

CONTRIBUTION OF TRANSIENT RECEPTOR POTENTIAL ANKYRIN-1

By

Lexiao Jin

M.S Wenzhou Medical University, 2008

M.D Wenzhou Medical University, 2003

Dissertation Approved on

$03-02-2020$

By the following Dissertation Committee

Daniel J. Conklin, Ph.D.

Aruni Bhatnagar, Ph.D.

Sanjay Srivastava, Ph.D.

John Pierce Wise, Ph.D.

Lu Cai, M.D., Ph.D.

Gary W. Hoyle, Ph.D. 


\section{DEDICATION}

This dissertation is dedicated to my family who have given me educational opportunities. 


\section{ACKNOWLEDGEMENTS}

First and foremost, I would like to thank my mentor, Dr. Conklin, for always believing in my scientific abilities, for his guidance, patience and support in my research. And he always pushes me to be my best and think critically. I would also like to thank my committee members, Dr. Bhatnagar, Dr. Srivastava, Dr. Hoyle, Dr. Wise, and Dr. Cai, for their comments and assistance in completing my dissertation research.

I must thank my husband and my parents who have always understood and encouraged me. And I especially thank my parents, they take turns to help me take care of my daughter. And I thank my friends, especially Hongxue Shi and Jian Jin, who always help me in my life. I thank my colleagues, Gregg, Luping, Jack, Jordan, Andre, Whitney, and Lexi, we always collaborate efficiently and happily. 


\begin{abstract}
THE CARDIOVASCULAR EFFECTS OF TOBACCO PRODUCT-DERIVED ALDEHYDES: ROLE OF TRPA1

Lexiao Jin
\end{abstract}

03-02-2020

\title{
Background
}

Cardiovascular diseases (CVD) are the leading cause of death in most countries and are a significant health and financial burden worldwide (Bhatnagar, 2004; Ramana et al., 2006). Smoking contributes to more than 480,000 premature deaths annually -- accounting for one of every five deaths in the United States each year (Centers for Disease Control and Prevention, 2018; U.S. Department of Health and Human Services, 2014). Numerous studies have shown that smoking increases the risk of factors including endothelial dysfunction, thrombosis, and atherosclerosis, that may lead to incidence of myocardial infarction, coronary artery disease, and stroke (Srivastava et al., 2011; U.S. Department of Health and Human Services, 2014). Electronic cigarettes (e-cigarettes) are a new kind of tobacco product, and some studies show that e-cigarettes also could induce CVD since both e-cigarettes and conventional tobacco products release many aldehydes. Extensive 
epidemiological and experimental data show that aldehydes contribute to cardiovascular morbidity and mortality. However, whether these aldehydes contribute to the adverse effects of emerging tobacco-products such as ecigarettes are unknown. The transient receptor potential ankyrin1 (TRPA1) channel is a receptor of unsaturated aldehydes that mediates tobacco smokeinduced lung injury in some animal models (Andre et al., 2008; Cheah, Burcham, Mann, \& Henry, 2014; D. J. Conklin, 2016; D. J Conklin, Haberzettl, Jagatheesan, Kong, \& Hoyle, 2017; Simon \& Liedtke, 2008). Thus, our central hypothesis is that aldehydes derived from e-cigarettes or conventional tobaccos contribute to vascular toxicity through the activation of TRPA1 pathway.

\section{Methods}

Both in vivo and in vitro studies were performed using male and female C57BL/6J mice. For in vivo studies, mice were exposed to aldehyde gas (1-5 ppm, 6 h/day) for 4 days or 12 weeks ( 6 h/day, 5 days/week). Non-invasive blood pressure and heart rate were measured weekly for 12 weeks by tail cuff system. Measure real-time blood pressure and heart rate by telemetry system. After exposures, we collected aortas and examined vascular function ex vivo, specifically, testing for any changes in sensitivity and efficacy of the aorta in response to pharmacological agents: phenylephrine (PE; contractions), acetylcholine (ACh; relaxation) and sodium nitroprusside (SNP; relaxation). PEinduced isometric contractions $(\mathrm{mN})$ were normalized by segment length and/or volume to compare aortic contractility. Relaxations were calculated as a 
percentage reduction in PE-induced tension. Sensitivity of each agent was

calculated as the effective concentration producing $50 \%$ response $\left(\mathrm{EC}_{50}\right)$.

In our in vitro studies, we examined the effects and underlying mechanisms of action (relaxation) of aldehydes in both isolated mouse superior mesenteric artery (SMA) and aorta. To test specific pathways, we used pharmacological inhibitors of nitric oxide synthase (NOS), guanylyl cyclase, TRPA1, and $\mathrm{K}^{+}$channels as well as mechanical injury of the endothelium.

\section{Results}

In our in vivo studies, C57BL/6J (wild type, WT) mice were exposed to HEPA-filtered room air (control), formaldehyde (FA; $5 \mathrm{ppm}$ ) or acetaldehyde (AA; 5ppm) for 4 days. There were no differences in the relaxations of ACh and SNP or in the PE-induced contractility of between control and aldehyde-exposed groups. So, short-term exposure to FA or AA at levels present in tobacco product aerosols did not alter aortic function. Mice were exposed to crotonaldehyde (CR) or filtered room air for 12 weeks ( 1 ppm, 6h/day, 5 d/week). Non-invasive blood pressure was measured by tail cuff (once a week) for 12 weeks. Blood pressures (systolic, mean, diastolic) were significantly lower in the CR group than in the air group over the last several weeks, yet there was no difference in heart rate between these two groups. Blood pressure changes were accompanied by changes in aortic vasoreactivity ex vivo. Following 12-wk CR chronic exposure, the sensitivity of ACh-induced relaxation was significantly increased in the CR 
group, however, there was no change in aortic response to PE. Similarly, aortic sensitivity to SNP relaxation was enhanced by CR compared with air control group. To dissect the mechanism of CR-induced vascular changes, we performed acute 4-day exposures ( 1 and 3 ppm). Similarly, CR exposure also altered aortic responses to ACh and slightly to SNP. Because a 4-day CR exposure was sufficient to alter aortic reactivity, we tested whether the transient receptor potential ankyrin 1 (TRPA1) channel was involved. In TRPA1-null mice exposed to CR (4-days, $1 \mathrm{ppm})$, there were no changes in aortic reactivity to any agonist (PE, ACh, and SNP) compared with air group, indicating a distinct role of TRPA1 in CR-induced changes. Interestingly, there also were no differences in any aortic responses in CR- (1 ppm 4 days) versus air-exposed female WT mice.

For the in vitro studies, we explored the direct effects of FA, AA and CR in isolated murine superior mesenteric artery (SMA) and aorta. FA (30-1,200 mM) and $A A(1-100 \mathrm{mM})$ strongly and reversibly relaxed agonist-induced contractions of SMA including phenylephrine (PE), thromboxane $A_{2}$ analog $(U 46,619)$ and high potassium (high $\mathrm{K}^{+}, 60 \mathrm{mM}$ ) without toxicity. The sensitivity but not the efficacy of AA-, FA- and CR-induced relaxations was dependent on blood vessel (wherein SMA was more sensitive than aorta) and contractile agonist (PE; U46,619; high $\mathrm{K}^{+}$) yet independent of circadian cycle and sex. All three aldehydeinduced relaxations were significantly inhibited (at low concentrations) in the presence of: 1) mechanically-impaired endothelium; 2) Nw-Nitro-L-arginine methyl ester hydrochloride (L-NAME); and 3) guanylyl cyclase (GC) inhibitor (ODQ). Positive immunofluorescent staining for TRPA1 was co-localized in 
endothelium of isolated SMA and the TRPA1-specific gene sequence was detected in SMA. The relaxations of FA and CR but not AA at low concentrations were all inhibited in the presence of TRPA1 antagonist (A967079).

\section{Conclusion}

These studies indicate that short-term exposure of mice to FA or AA have limited direct toxicity in aorta, however, CR exposure (acute and chronic) leads to altered vascular function that may lead to vascular disease. Moreover, we describe direct effects of $F A, A A$, and $C R$ that are dependent on an EDRF/NO and VSMC GC/PKG pathway in SMA, with FA- and CR-induced relaxations also dependent on an endothelium-localized TRPA1 receptor. From our vitro study, we conclude that aldehydes play a key role in the regulation of blood flow in the gastrointestinal (Gl) tract to augment digestion and nutrient absorption and induce postprandial hyperemia, and TRPA1 receptor plays an important role in the aldehyde-induced vasodilation in vasculature. Future studies are required to better understand how CR exposure modifies cardiovascular pathophysiology, and the specific roles of TRPA1 and sex play in contributing to burden of tobacco-related CVD including pathophysiological processes of atherosclerosis. 
TABLE OF CONTENTS

PAGE

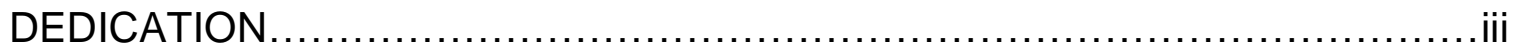

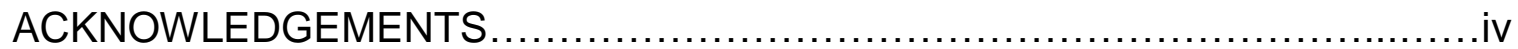

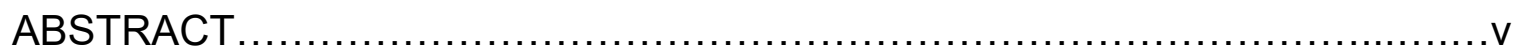

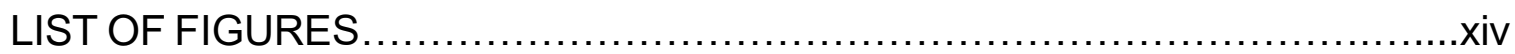

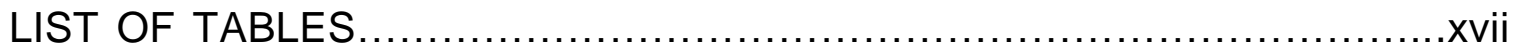

CHAPTER I INTRODUCTION

Background and the Rationale..............................................

Conventional tobacco smoking and cardiovascular disease (CVD) .........1

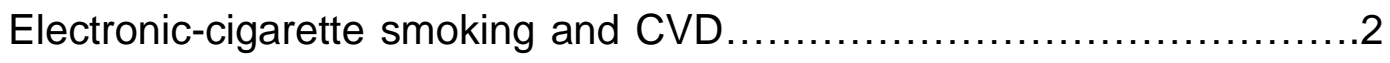

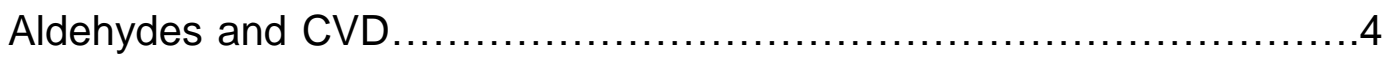

Endothelium Dysfunction and CVD .....................................

Transient receptor potential ankyrin1 (TRPA1) and CVD..................

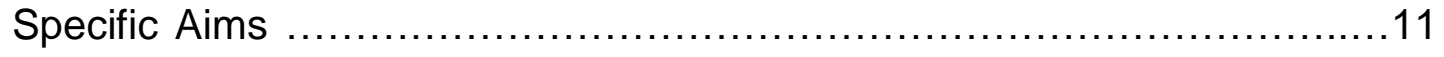

Aim 1: Determine the direct vascular effects of tobacco-product derived

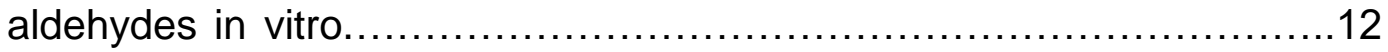

Aim 2. Determine the vascular effects (endothelium dysfunction) of tobaccoproduct derived aldehydes exposures in vivo $\ldots \ldots \ldots \ldots \ldots \ldots \ldots 12$

Aim 3. Are vascular effects of aldehydes mediated by TRPA1? ..............13 
CHAPTER $\square$ A SIMPLE METHOD FOR NORMALIZATION OF AORTIC

\section{CONTRACTILITY}

Introduction

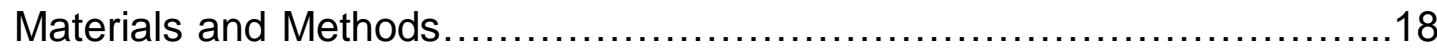

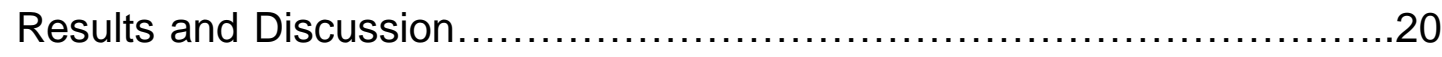

CHAPTER III FORMALDEHYDE INDUCES MESENTERIC ARTERY RELAXATION VIA A SENSITIVE TRANSIENT RECEPTOR POTENTIAL ANKYRIN-1 (TRPA1) AND ENDOTHELIUM-DEPENDENT MECHANISM: POTENTIAL ROLE IN POSTPRANDIAL HYPEREMIA

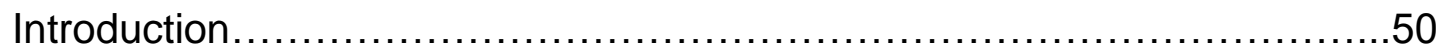

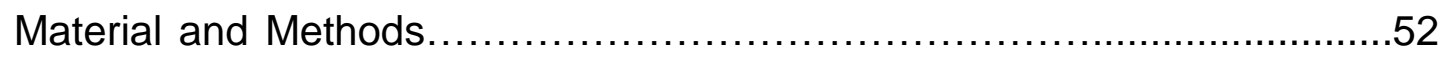

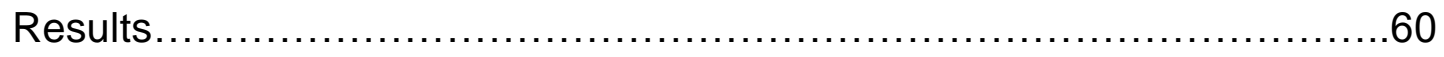

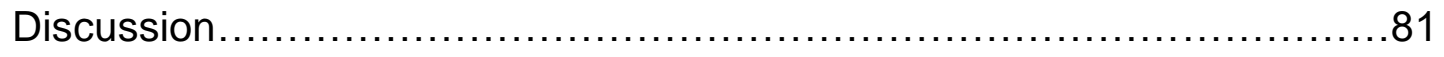

CHAPTER IV ACETALDEHYDE INDUCES AN ENDOTHELIUM-DEPENDENT RELAXATION OF SUPERIOR MESENTERIC ARTERY: POTENTIAL ROLE IN POSTPRANDIAL HYPEREMIA

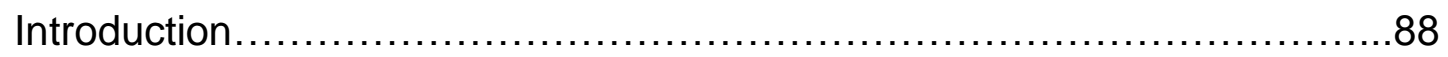

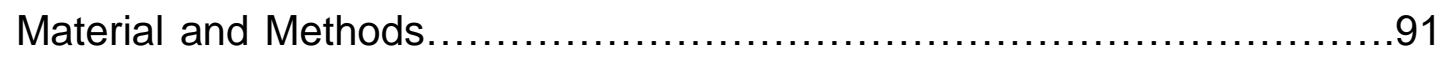

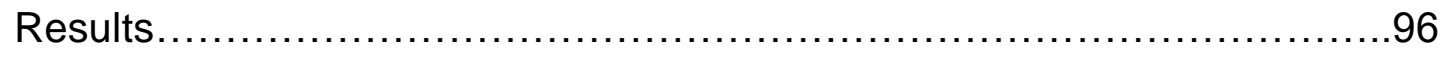


Discussion

CHAPTER V CROTONALDEHYDE-INDUCED VASCULAR RELAXATION AND TOXICITY: ROLE OF ENDOTHELIUM AND TRANSIENT RECEPTOR POTENTIAL ANKYRIN-1 (TRPA1)

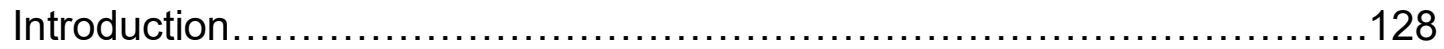

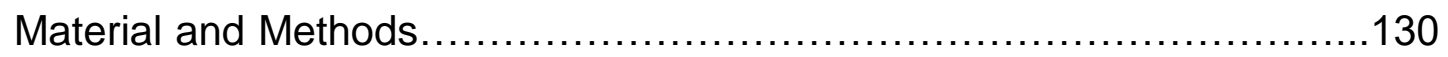

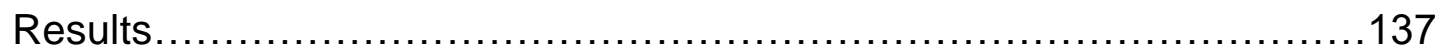

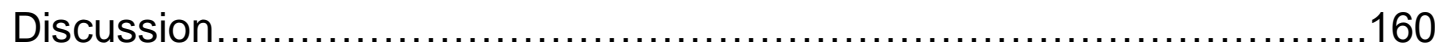

CHAPTER VI THE CARDIOVASCULAR EFFECTS OF INHALED ALDEHYDES IN VIVO: ROLE OF TRANSIENT RECEPTOR POTENTIAL ANKYRIN-1 (TRPA1)

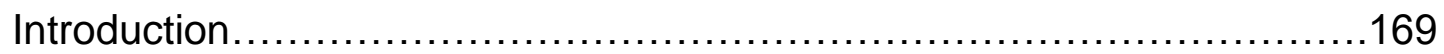

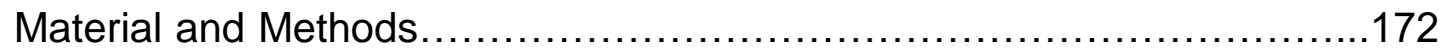

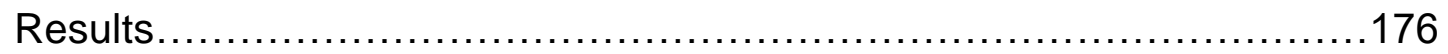

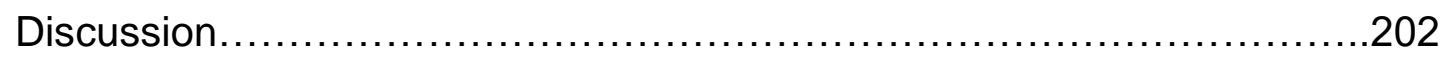

CHAPTER VII SUMMARY AND CONCLUSIONS

Major findings in this dissertation......................................211

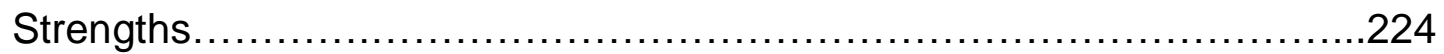

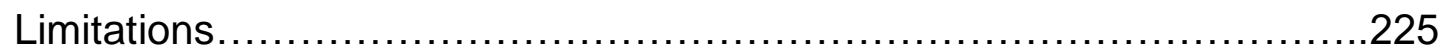

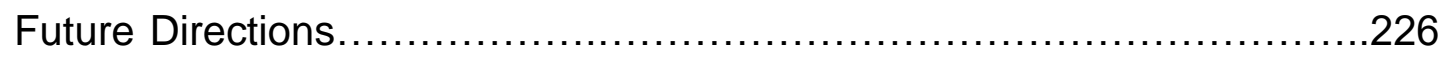


Conclusions.

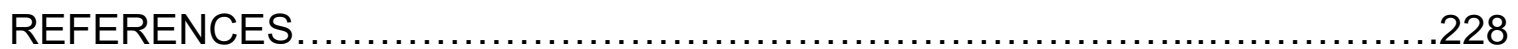

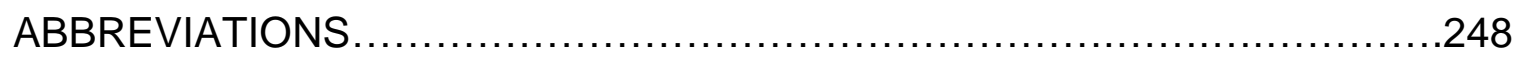

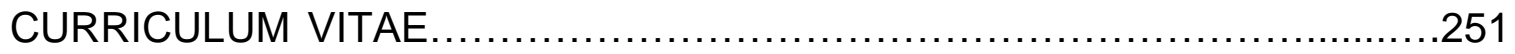




\section{LIST OF FIGURES}

FIGURE

PAGE

\section{CHAPTER II}

Figure. 1. Images of method for aortic parameter data acquisition...............21

Figure. 2. Normalization of vascular contractility data.........................24

Figure. 3. Normalization of vascular contractility of aortas from C57BL/6 mice fed normal chow (NC; $n=9$ mice) or high fat diet (HFD; $n=10$ mice) for 12 weeks...29

Figure. 4. Normalization of vascular contractility of aortas from Sprague-Dawley wild type (WT; $n=5$ rats) and LDLR-KO rats ( $n=6$ rats) fed normal chow for 1 year.

Figure. 5. Reproducibility of aorta parameter data acquisition: intra-assay variation.

Figure. 6. Reproducibility of aorta parameter data acquisition: inter-assay variation.

Figure. 7. Intra-aortic variation in cross-sectional area........................43

Figure. 8. Resiliency of aorta parameter data acquisition over time 45

\section{CHATPER III}

Figure. 9. Formaldehyde (FA) induced relaxation in superior mesenteric artery (SMA).

Figure. 10. Regulators of agonist-induced tension in superior mesenteric artery (SMA).

Figure. 11. Role of the endothelium and nitric oxide (NO) in the most sensitive component of formaldehyde-induced relaxation of phenylephrine (PE) precontracted superior mesenteric artery (SMA)

Figure. 12. Role of the TRPA1 channel in formaldehyde (FA)-induced relaxation in phenylephrine (PE) pre-contracted superior mesenteric artery (SMA). 
Figure. 13. Role of the endothelium and nitric oxide (NO) in the most sensitive component of cinnamaldehyde-induced $(\mathrm{CA})$ relaxation of phenylephrine (PE) pre-

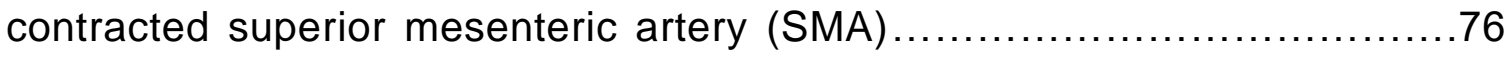

Figure. 14. Role of potassium channel (KIR) in formaldehyde (FA)-induced relaxation in phenylephrine (PE) pre-contracted blood vessels. 78

Figure. 15. A cartoon depicting the mechanisms by which formaldehyde (FA) induces vasorelaxation in SMA precontracted by phenylephrine (PE), thromboxane A2 analog (U46,619) and $60 \mathrm{mM}$ potassium solution (60K) 85

\section{CHAPTER IV}

Figure. 16. Acetaldehyde (AA) induced relaxation in superior mesenteric artery (SMA).

Figure. 17. Role of the endothelium and nitric oxide (NO) in acetaldehyde-induced (AA) relaxation of SMA 105

Figure. 18. Role of the nitric oxide (NO) in acetaldehyde-induced relaxations of SMA. 109

Figure. 19. The vascular effects of ethanol (EtOH), acetaldehyde (AA) and acetate in SMA. Role of the nitric oxide (NO) in ethanol- and acetate-induced relaxations of SMA... 111

Figure. 20. The vascular effects of ethanol (EtOH), acetaldehyde (AA) and acetate in aorta...

Figure. 21. Role of aldehyde dehydrogenase (ALDH) metabolism in vascular responses to $\mathrm{EtOH}, \mathrm{AA}$ and acetate.

Figure. 22. Mechanisms of acetaldehyde-induced vasorelaxation and relationship to aldehyde metabolism.

\section{CHAPTER V}

Figure. 23. Crotonaldehyde-induced (CR) relaxation in aorta and superior mesenteric artery (SMA). 141

Figure. 24. Mechanisms of crotonaldehyde-induced (CR) relaxation in superior mesenteric artery (SMA). 146

Figure. 25. Role of the TRPA1 channel in crotonaldehyde (CR)-induced relaxation in superior mesenteric artery (SMA) 148 
Figure. 26. Role of TRPA1 in AITC-induced relaxation of superior mesenteric artery (SMA), and localization in SMA and aorta. 151

Figure. 27. Immunofluorescent localization of TRPA1 in dorsal root ganglion (DRG). 153

Figure. 28. Toxicity of crotonaldehyde (CR) exposure in superior mesenteric artery (SMA) 155

Figure. 29. Effects of nicotine on CR-induced relaxation in SMA. 158

Figure. 30. Cartoon depicting the mechanisms of crotonaldehyde $(C R)$ induced vasorelaxation in SMA. 164

\section{CHAPTER VI}

Figure. 31. Hemodynamic effects of inhaled crotonaldehyde (CR) in mice.

Figure. 32. Vascular toxicity of chronic crotonaldehyde (CR) inhalation exposure in mice.

Figure. 33. Vascular toxicity of acute crotonaldehyde (CR) inhalation exposure in C57BL/6 mice. 186

Figure. 34. Vascular toxicity of acute crotonaldehyde $(\mathrm{CR})$ inhalation exposure in C57BL/6 mice. 188

Figure. 35. Vascular toxicity of acute crotonaldehyde $(\mathrm{CR})$ inhalation exposure in TRPA1-null mice. 192

Figure. 36. Vascular toxicity of acute crotonaldehyde (CR) inhalation exposure female mice. 194

Figure. 37. The changes of aorta function after 4 days of exposure of FA 5 ppm 196

Figure. 38. The changes of aorta function after 2 weeks of exposure of AA 5 ppm.

Figure. 39. Vascular smooth muscle toxicity of aldehydes and endothelium function change after aldehydes exposure 208 


\section{LIST OF TABLES}

TABLE

PAGE

\section{CHAPTER II}

Table 1. Thoracic aorta segment parameters from C57BL/6 mice fed normal chow $(n=9)$ or high-fat diet $(n=10)$ for 12 weeks.................................

Table 2. Normalization of high potassium-induced $(100 \mathrm{mM})$ isometric contractions in thoracic aortas isolated from male mice fed either normal chow (NC) or high fat

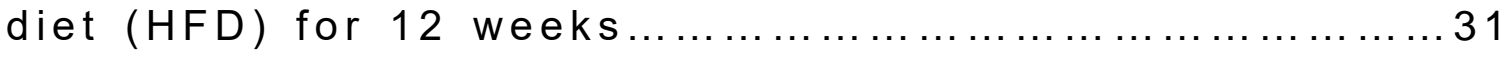

Table 3. Thoracic aorta segment parameters from wild-type (WT) and LDLR-null (LDLR-KO) 1-year-old Sprague-Dawley rats.

Table 4. Normalization of PE-induced $(10 \mu \mathrm{M})$ isometric contractions in thoracic aortas isolated from wild-type (WT) and LDLR-null (LDLR-KO) 1-year-old SpragueDawley rats

\section{CHATPER III}

Table 5. Levels of urinary formate following either a daytime fast or overnight feeding in male C57BL/6J mice

Table 6 . The effective concentration inducing $50 \%$ relaxation $\left(\mathrm{EC}_{50}\right.$; in $\left.\mu \mathrm{M}\right)$ of formaldehyde-induced vasorelaxation in isolated murine superior mesenteric artery (SMA; male and female; day and night) and thoracic aorta precontracted with different agonists 68

Table 7. Efficacy of formaldehyde and associated compounds in relaxation of PEprecontracted superior mesenteric artery (SMA) and aorta without and with selective treatments (inhibitors).

Table 8. The sensitivity of formaldehyde-induced vasorelaxation measured as the effective concentration inducing $50 \%$ relaxation $\left(\mathrm{EC}_{50}\right.$; in $\left.\mu \mathrm{M}\right)$ in isolated murine superior mesenteric artery (SMA; male) pre-contracted with PE in the absence and presence of functional endothelium and selective inhibitors of mediators of vasorelaxation 80 


\section{CHAPTER IV}

Table 9. Levels of urinary acetate following a daytime fast and overnight feeding in $(A)$ female and $(B)$ male $C 57 \mathrm{BL} / 6 \mathrm{~J}$ mice 101

Table 10. The effective concentration inducing $50 \%$ relaxation (EC50; in $\mathrm{mM}$ ) of acetaldehyde-induced vasorelaxation in isolated murine superior mesenteric artery (SMA; female and male) and thoracic aorta (male only) with different contractile agonists. 102

Table 11. Efficacy (maximal relaxation, Emax) of acetaldehyde- (AA), EtOH- and acetate-induced relaxations of PE-precontracted superior mesenteric artery (SMA) and aorta without and with inhibitor treatments 107

Table 12. The effective concentration inducing $50 \%$ relaxation (EC50; in $\mathrm{mM}$ ) of acetaldehyde- (AA), EtOH- or acetate-induced vasorelaxation in isolated murine superior mesenteric artery (SMA; male mice only) pre-contracted with PE in the absence and presence of functional endothelium or selective inhibitors/compounds. 108

Table 13. Role of aldehyde dehydrogenase (ALDH) in AA-induced relaxations of PE-precontracted SMA. 120

\section{CHAPTER V}

Table 14. Efficacy (maximal relaxation, Emax) of crotonaldehyde-induced relaxations of PE-precontracted superior mesenteric artery (SMA; male and female) without and with inhibitor treatments

Table 15. The sensitivity (effective concentration inducing 50\% relaxation; EC50; in $\mu \mathrm{M}$ ) of crotonaldehyde (CR)-induced vasorelaxation in PE pre-contracted murine superior mesenteric artery (SMA) in the absence and presence of functional endothelium or selective inhibitors. 144

Table 16. Characteristics of tension oscillations induced by a high concentration of crotonaldehyde $(\mathrm{CR} ; 300 \mu \mathrm{M})$ in PE-precontracted superior mesenteric artery (SMA). 145

Table 17. Vasotoxicity of crotonaldehyde (CR) in superior mesenteric artery (SMA). 157

CHAPTER VI 
Table 18. Echocardiography measures in male mice chronically exposed to air or crotonaldehyde.

Table 19. Effects of either air or crotonaldehyde (CR; 1 or $3 \mathrm{ppm}$ ) exposure on efficacy responses of isolated thoracic aorta of C57BL/6J (wild type, WT) and TRPA1-null mice to pharmacological agents in the absence and presence of nitric oxide synthase inhibitor (L-NAME) 190

Table 20. Effects of either air or crotonaldehyde (CR; 1 or $3 \mathrm{ppm}$ ) exposure on sensitivity responses of isolated thoracic aorta of C57BL/6J (wild type, WT) and TRPA1-null mice to pharmacological agents. 191

Table 21 . The effective concentration inducing $50 \%$ relaxation (EC50) of aldehydeinduced vasorelaxation in isolated murine (male) superior mesenteric artery (SMA) with different contractile agonists in the absence and presence of the nitric oxide

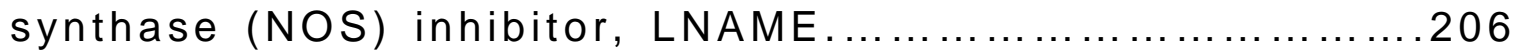




\section{CHAPTER I}

\section{INTRODUCTION}

\section{Background and Rationale}

\section{Conventional tobacco smoking and cardiovascular disease (CVD)}

Cardiovascular diseases (CVDs) are the leading cause of death in most countries and it kills 1 million people per year; accounting for over $40 \%$ of all deaths (Levy D, 2000). More than 900000 deaths for the reason of CVD in the United States in 2016 (Roth et al., 2018). It is a significant health and financial burden worldwide (Bhatnagar, 2004, 2006). In 2011, the United States spent $\$ 316.6$ billion directly and indirectly on CVD, more than the cost of other diagnostic group (Writing Group et al., 2019), and the total cost of CVD is increased exponentially over the past two decades (Heidenreich et al., 2011). Based on these data, looking for the reason and the way of prevention for the CVD showed to be urgent to humans.

Smoking has been used for thousands of years in the worldwide and has been studied for several decades. In 2017, the number of smoker exceeded 1 billion globally and the Centers for Diseases Control and Prevention (CDC) reported that $14 \%$ of U.S. adults were current smokers (Centers for Disease Control and Prevention, 2017). In 2018, 27.1\% of high school students and $7.2 \%$ 
middle school students were current tobacco users (Centers for Disease Control and Prevention, 2018). In 2017, the World Health Organization (WHO) reported that tobacco caused about 8 million global deaths each year (World Health Organization, 2019). In the United States, Smoking kills more than 480,000 deaths annually and accounted for one of every five deaths in the United States each year (Centers for Disease Control and Prevention, 2018; U.S. Department of Health and Human Services, 2014). Numerous studies showed that smoking increased the risk of thrombosis, atherosclerosis, etc. which narrowed the vascular lumen and caused myocardial infarction, coronary artery disease and stroke (Srivastava et al., 2011; U.S. Department of Health and Human Services, 2014). Cigarette smoking also is a cause of peripheral arterial disease (PAD), aortic aneurysm, and cerebrovascular disease. Over $90 \%$ of patients with symptomatic PAD have a chronic smoking history (Hughson, Mann, \& Garrod, 1978; Powell et al., 1997). About $11 \%$ of Americans are affected by PAD, $11.08 \%$ developed to limb ischemia (Nehler et al., 2014). PAD affects $22.7 \%$ of those aged $>80$ years, especially $59 \%$ in male African American are affected (Benjamin et al., 2019; Writing Group et al., 2019).

\section{Electronic-cigarette smoking and CVD}

Except for conventional tobacco smoking, there are more and more new forms of smoking, such as electronic cigarette, hookahs, JUUL, etc... Electronic cigarette is a new kind of tobacco product that was introduced to the commercial market in 2004. E-cigarette includes aerosol delivery devices and a battery, the 
cartridge contains e-liquids that contain a mixture of glycerol and propylene glycol, flavors, and most commonly with variable concentrations of nicotine ( $T$. Cheng, 2014; Hutzler et al., 2014) .

As e-cigarettes initially were exclaimed to be less toxic for the smoker's health, there has been a steady increase in e-cigarette users especially among high school students, wherein e-cigarette users increased from $1.5 \%$ in 2011 to 20.8\% in 2018 (Cullen et al., 2018; Gentzke et al., 2019). More and more research shows the potential harm to e-cigarette users (Benowitz \& Fraiman, 2017; Bhatnagar et al., 2019). In animal studies, Olfert et al. (2018) showed that mice were caused cardiovascular dysfunction such as impaired vascular function and increased arterial stiffness after had been exposed to e-cigarette aerosol for 8 months (Olfert et al., 2018). Another study showed that e-cigarette exposure induces cardiac dysfunction and atherosclerosis in apolipoprotein-E-null mice (Espinoza-Derout et al., 2019). Two human studies showed that sympathetic activity and oxidative stress significantly increased in e-cigarette users (Carnevale et al., 2016; Moheimani et al., 2017). Another one clinical study showed that e-cigarette aerosol could suppress the immune system and inflammatory response genes in nasal epithelial cells similar to cigarette smoke (Martin et al., 2016).

E-cigarettes do not induce combustion as in conventional tobacco smoking. During heating of e-cigs, the humectants (VG, PG) are thermally decomposed and oxidized into short, toxic aldehydes including formaldehyde, acetaldehyde, and acrolein (D. J. Conklin et al., 2018; Ogunwale et al., 2017). 
These aldehydes are well associated with endothelium dysfunction of cigarette smoking (Anderson, Majeste, Hanus, \& Wang, 2016; G. E. Hill et al., 1998; S. E. Lee \& Park, 2013; Lino-dos-Santos-Franco et al., 2011; Lino-dos-Santos-Franco et al., 2010; Park \& Taniguchi, 2008). What's more, different brands of ecigarettes contain varying amounts of nicotine along with multiple other elements in the smoke of the e-cigarette (Hahn et al., 2014). Numerous studies showed the cardiovascular effects of nicotine. During smoking, nicotine stimulates the release of catecholamines, thereby increases heart rate and blood pressure (Benowitz \& Gourlay, 1997; Benowitz, Hansson, \& Jacob, 2002). Nicotine could also increase platelet aggregation (Hom et al., 2016), induce vascular smooth muscle cell proliferation (Egleton, Brown, \& Dasgupta, 2009), and increase atherosclerosis (Santanam et al., 2012).

We still do not know much about the risks of e-cigarettes to the smoker or second-hand smoker or the extent of harm. Many health care providers continue to urge people to be cautious in considering this as an alternative to cigarette smoking.

\section{Aldehydes and CVD}

Aldehydes are toxic components of automobiles exhaust and smoke, and have been detected in high concentrations in cigarette, cotton, wood, and coal smoke. Aldehydes are even present in drinking water. Cooking and frying further 
would increase aldehydes concentration in foods. The metabolism of several pollutants and toxicants also generates aldehydes.

Extensive epidemiological and experimental data show that aldehydes contribute to cardiovascular mortality and morbidity. Lots of our previous studies showed the effects of aldehydes on lipid metabolism, atherosclerosis, platelet activation, thrombosis, myocardial function, and sensitivity to ischemiasuggesting that environmental aldehydes could increase CVD (D. J. Conklin, R. A. Prough, et al., 2011; B. G. Hill, Haberzettl, Ahmed, Srivastava, \& Bhatnagar, 2008; Makia, Bojang, Falkner, Conklin, \& Prough, 2011; O'Toole et al., 2014; Sithu et al., 2010; Srivastava et al., 2011; Srivastava et al., 2009). Some aldehydes, such as acrolein, acetaldehyde, and formaldehyde are considered the most significant cardiovascular and pulmonary toxins in tobacco smoke (Haussmann, 2012). Wheat et al. found acrolein inhalation exposure suppressed circulating endothelial progenitor cell (EPC) levels, indicating that EPC depletion might be a significant contributory factor linking pollutant exposure to CVD risk (Wheat et al., 2011). Other studies showed that e-cigarette aerosol including $\alpha, \beta$ unsaturated aldehydes and saturated aldehydes led to inflammatory changes such as induction of cyclooxygenase-2 and cytokines, interacted with endothelium-leukocyte, increased lung vascular permeability, induced reactive oxygen species, DNA damage, apoptosis and programmed necrosis pathways in vascular endothelial cells (Anderson et al., 2016; S. E. Lee \& Park, 2013; Linodos-Santos-Franco et al., 2011; Lino-dos-Santos-Franco et al., 2010). However, whether these aerosolized aldehydes contribute to the adverse effects of e- 
cigarette that could induce endothelium dysfunction via its produced aldehydes is still unclear.

The metabolism of several industrial pollutants such as butadiene, vinyl chloride, benzene, and allylamine, generate aldehydes that may be the common metabolite responsible for the cardiotoxicity of these pollutants. For example, acrolein and crotonaldehyde are also formed during the metabolism of 1,3butadiene (Nauhaus, Fennell, Asgharian, Bond, \& Sumner, 1996; Richardson et al., 1998), which is used in the rubber industry. Butadiene is also a reactive constituent of cigarette smoke (Himmelstein, Acquavella, Recio, Medinsky, \& Bond, 1997). Occupational exposure to butadiene is associated with a significant risk of death from arteriosclerotic heart disease (Matanoski, Santos-Burgoa, \& Schwartz, 1990). Moreover, butadiene exposure has been shown to increase atherosclerosis in cockerels (Penn \& Snyder, 1996a, 1996b) and generation of hemangiosarcomas in the heart (Solleveld, Miller, Banas, \& Boorman, 1988). Aldehydes are metabolized in cardiac myocytes, smooth muscle cells and endothelial cells by glutathione-S-transferases (GSTP) and aldehyde dehydrogenase (ALDH) (D. J. Conklin et al., 2004; D. J. Conklin, Haberzettl, et al., 2015b; D. J. Conklin, Haberzettl, Prough, \& Bhatnagar, 2009b). Formaldehyde is metabolized to formate, and acetaldehyde is metabolized to acetate. Those two metabolites are extremely high in the urine (D. J. Conklin et al., 2018; D. J. Conklin, S. Schick, et al., 2019). Our preliminary data showed that formate had no relaxation in precontracted aorta, formate and acetate had some 
relaxation in superior mesenteric artery (SMA), but significantly lower than formaldehyde and acetaldehyde (Jin, Jagatheesan, et al., 2019).

Toxic metabolites of aldehydes and other environmental pollutants may actually be contributing to cardiovascular effects in vivo. Since many environmental pollutants are metabolized to aldehydes, research into the mechanisms of how aldehydes induce CVD could help study the mechanisms of these environmental pollutants as parent compounds. Rewrite. However, there was still a long way to study how aldehydes impair cardiovascular function or enhance cardiovascular diseases.

\section{Endothelium Dysfunction and CVD}

Endothelium is important in the regulation of vascular tone through the production of a variety of mediators such as NO, ROS, prostanoids, and endothelin. It acts as an active signal transducer instead of as a barrier (Vita \& Loscalzo, 2002). Bunches of studies showed that smoking could directly damage to endothelial cells and affect endothelial function (Barua, Ambrose, Srivastava, DeVoe, \& Eales-Reynolds, 2003; Messner \& Bernhard, 2014; Raij, DeMaster, \& Jaimes, 2001). Alteration in endothelial function causes the initiation of prothrombotic, proinflammatory and proliferative states and induces the development of morphological atherosclerotic changes. Endothelial dysfunction is the first step in vascular disease, since it leads to vascular inflammation, cell proliferation, and thrombosis, seems to be the most notable indicator of the 
progression of cardiovascular disease (CVD), leading to high morbidity and mortality from CVD (Daiber et al., 2017; Huveneers, Daemen, \& Hordijk, 2015; Lerman \& Zeiher, 2005; Schachinger, Britten, \& Zeiher, 2000; Yannoutsos, Levy, Safar, Slama, \& Blacher, 2014). Evaluation of endothelial function is crucial to our study about the vascular effects of aldehydes.

Endothelial dysfunction refers to the impairment of endotheliumdependent vasodilatation, which implies abnormalities in endothelial integrity and homeostasis and usually occurs at an early stage of hypertension (Deanfield, Halcox, \& Rabelink, 2007). The imbalance between the dilator and constrictor forces in the vasculature leads to injurious changes in the endothelium, these changes are considered as an evidence of dysfunction and as a sign of unhealthy vasculature (Kolluru, Bir, \& Kevil, 2012).

Endothelial cells synthesize and secrete a broad of anti-atherosclerotic substances, the principal of which is nitric oxide (NO) that is generated from the metabolism of L-arginine by endothelial NO synthase (eNOS), constitutively expressed in endothelial cells (Luscher, 1990). Endothelial dysfunction is defined as a reduced NO bioavailability. Endothelial progenitor cells (EPCs) have multiple different cell types that play roles in the regeneration of the endothelial lining of blood vessels. They are released from the bone marrow to the sites of endothelial damaged blood vessels and promote an angiogenesis, which play an important role in the proper endothelial function (Asahara et al., 1999; Pelosi, Castelli, \& Testa, 2014). The levels of endothelial progenitor cells might be a surrogate biologic marker for vascular function and cumulative cardiovascular 
risk (Vasa et al., 2001). An increase in EPC levels was also shown to correlate with the occurrence of ischemic stroke (Liao et al., 2017).

\section{Transient receptor potential ankyrin1 (TRPA1) and CVD}

Despite this high disease burden, we still know little about the mechanisms by which smoking causes CVD. The transient receptor potential family is a large family of cation selective ion channels that are extensively expressed in the sensory nerves, endothelium and vascular smooth muscle cells. Transient receptor potential ankyrin1 (TRPA1) is a promiscuous sensory receptor that is activated by a variety of environmental and endogenous noxious compounds, including $\alpha, \beta$-unsaturated aldehydes such as acrolein (Bautista et al., 2006; Cheah et al., 2014; D. J. Conklin, 2016; D. J Conklin et al., 2017), cinnamaldehyde (Bandell et al., 2004; Bodkin \& Brain, 2011; Pozsgai et al., 2010), and 4-hydroxynonenal (4HNE) (Trevisani et al., 2007). TRPA1 mediates pain-reception/transmission (nociceptors). Recently, the distribution of TRPA1 receptor has been expanded to include non-neuronal locations, such as the heart, endothelium, and urothelium (Bautista et al., 2006; Earley, Gonzales, \&

Crnich, 2009a; Kolluru et al., 2012; Pelosi et al., 2014), it is also present in vasculature (Earley et al., 2009a; Sinharoy et al., 2017).

Activation of peripheral sensory fiber TRPA1 leads to pain signaling and likely triggers the opening of the cation channel, allowing calcium entry, neuronal activation, and release of vasoactive peptides, substance $\mathrm{P}$ (SubP) and 
calcitonin gene-related peptide (cGRP) (Macpherson et al., 2007; Trevisan et al., 2016), which may contribute to increased vasodilation. These peptides increase local tissue inflammation, blood flow, vascular permeability, edema and leukocyte binding and extravasation (Andrade, Meotti, \& Calixto, 2012). Thus, activation of the TRPA 1 channel by acrolein is linked with inflammation, which could contribute to acute tobacco smoking- or acrolein-induced injury in the airways and the cardiovascular system (Bautista et al., 2006; Earley et al., 2009a; Inoue et al., 2006). Our previous study showed that pre- and post-exposure TRPA1 antagonism significantly protected against acrolein-induced mortality showed a complex role of TRPA1 in cardiopulmonary injury (D. J Conklin et al., 2017). The airway-located TRPA1 triggers an irritant response (e.g., coughing and "respiratory braking") that alters the rate and depth of breathing to reduce exposure, what's more, the morbidity and mortality significantly increased in the TRPA1-null mice might be for the reason that these TRPA1-null were deficient in this "respiratory braking" (D. J Conklin et al., 2017). Cinnamaldehyde-induced dose-dependent blood vessel relaxation, blood pressure, heart rate and local blood flow was significantly less potent in TRPA1-null compared with wide type arteries (Pozsgai et al., 2010). However, the relationship between TRPA1 and ecigarettes aerosol induced endothelial dysfunction and CVD risk is still unclear.

In summary, voluntary and involuntary exposures to cigarette smoke (and perhaps e-cigarette aerosols) are major causes of CVD such as CHD, stroke, aortic aneurysm, and PAD. Smoking releases aldehydes, and numerous studies show that aldehydes can also induce CVD. The risk is seen both as an increased 
risk of endothelial dysfunction and acute thrombosis of narrowed vessels and as an increased of atherosclerosis in the blood vessels involved. The cardiovascular risks attributable to cigarette smoking increase with the number of cigarettes smoked and the duration of smoking. The mechanisms of the smoking induce endothelial dysfunction are still unclear. TRP family play important roles in the development of CVD, especially TRPA1 receptor. It is important to clarify this mechanism in order to decrease the impact that cigarette products exposure has on the incidence of endothelial dysfunction and CVDs. Once this mechanism has been better clarified, and the toxicity of cigarette smoking or e-cigarette smoking has been identified, we can begin to develop health guidelines and pharmaceutical interventions to reduce the frequency of CVD both nationally and globally. A decreased rate of cigarette smoking and a decreased incidence of CVDs would have a significant impact on the cost of medical treatment and the worldwide financial burden and, more importantly, on the global quality of life.

\section{B. Specific aims in this study}

The primary goal of the proposed research is to determine whether the aldehydes derived from tobacco products (electronic cigarettes, conventional tobacco) may be harmful to the vasculature, and, if so, what is the underlying mechanism. Over-exposure to aldehydes is known to produce cardiovascular toxicity. However, whether these aldehydes contribute to the adverse effects of tobacco-products such as electronic cigarettes are unknown. The transient receptor potential ankyrin1 (TRPA1) channel is a receptor of aldehydes and 
mediates tobacco smoke-induced lung injury in some animal models. Thus, our central hypothesis is that tobacco product-derived aldehydes contribute to vascular toxicity through activation of the TRPA1 pathway. To test this hypothesis, we explored the following aims:

\section{Aim 1. Determine the direct vascular effects of tobacco-product derived aldehydes in vitro.}

We explored the possible pathways of vascular effects of aldehydes, and found that the relaxation of formaldehyde (FA), acetaldehyde (AA) and crotonaldehyde (CR) were all endothelium dependent, nitric oxide dependent, and cGMP dependent. We also showed that the relaxation of FA and CR was TRPA1 dependent. Furthermore, we tested the vascular effects of aldehydes metabolites. Formate (main metabolite of FA; up to $1 \mathrm{mM}$ ) nor methanol (a stabilizer in formalin, up to $1 \mathrm{mM}$ ) induced a weak relaxation in SMA indicating that FA-induced relaxation was not a result of formate or methanol. Ethanol and acetate could dilate blood vessels but much lower than AA and were all nitric oxide dependent. Because smoking is often inhaled with nicotine, we tested if nicotine modified aldehyde effects, but nicotine $[1 \mu \mathrm{M}]$ had no effect on subsequent $F A, A A$ and $C R$-induced vasorelaxation.

\section{Aim 2. Determine the vascular effects (endothelium dysfunction) of tobacco-product derived aldehydes exposures in vivo.}

We hypothesize that the of electronic cigarettes or conventional tobacco could cause cardiovascular injury through its aldehydes. Mice were exposed to 
FA (5ppm) or AA (5ppm) for 4 days, there was no significant change in aorta function after FA or AA exposure. Mice were exposed to $C R$ or air for 12 weeks (1 ppm, 6h/day, $5 \mathrm{~d} /$ week). Blood pressure was significantly lower in the CR group than in the air group for the last several weeks, but there was no difference in the changes of heart rate between these two groups. Hemodynamic changes were accompanied by changes in aortic vasoreactivity ex vivo. Following 12 -wk CR chronic exposure, there was no change in aortic response to phenylephrine $(\mathrm{PE})$, however, the sensitivity of $\mathrm{ACh}$-induced relaxation was significantly increased in the CR group. Similarly, aortic sensitivity to SNP relaxation was enhanced by CR. To dissect the mechanism of CR-induced vascular changes, we performed acute 4-day exposures ( 1 and 3 ppm). Similarly, CR exposure also altered aortic responses to ACh and somewhat to SNP.

\section{Aim 3. Are vascular effects of aldehydes mediated by TRPA1?}

We hypothesize that the aldehydes derived from tobacco products induce vascular injury might through the TRPA1 pathway. In our vivo study, since a 4day CR exposure was sufficient to alter aortic reactivity, we tested whether the transient receptor potential ankyrin 1 (TRPA1) channel was involved. In TRPA1null mice exposed to CR (4-days, $1 \mathrm{ppm}$ ), there were no changes in aortic reactivity to any agonist (PE, ACh, and SNP) compared with air group, indicating a distinct role of TRPA1. In our vitro study, since histology staining showed there were a lot of TRPA1 proteins in endothelium in superior mesenteric artery (SMA). We tested whether the relaxation of FA, AA or CR via TRPA1 pathway. TRPA1 antagonist (A967079) has significantly inhibited the relaxation of FA and CR at 
low concentrations in SMA. The relaxation of CR significantly decreased in TRPA1-null mice. We concluded that the relaxation of FA and CR are TRPA1 dependent.

We also have tested the sex dependence in the cardiovascular effects of aldehydes. In our vivo study, we tested a 4-day CR exposure to female mice, Interestingly, there were also no differences in any aortic responses in CR versus air-exposed female mice. For the in vitro study, there was no difference in the relaxation of $F A$ and $A A$ between male and female mice. The mechanism of female-dependent protection in mice is not clear. 


\section{CHAPER II}

\section{A SIMPLE METHOD FOR NORMALIZATION OF AORTA CONTRACTILITY}

Vascular contractile function changes in proliferative vascular diseases, e.g., atherosclerosis, and is documented using isolated blood vessels, yet, many laboratories differ in their approach to quantification. Some use raw values (e.g., $\mathrm{mg}, \mathrm{mN}$ ); others use a 'percentage of control agonist' approach; and, others normalize by blood vessel characteristic, e.g., length, mass, etc. A lack of uniformity limits direct comparison of contractility outcomes. To address this limitation, we developed a simple 2-step normalization method: 1) measure blood vessel segment length $(\mathrm{mm})$ and volume $\left(\mathrm{mm}^{3}\right)$; and, 2) normalize isometric contraction $(\mathrm{mN})$ by segment length and/or volume. Normalization reduced variability, and thus, normalized aortic contractions but not raw values were statistically different between normal chow (NC) and high fat diet-fed (HFD) mice supporting the practical utility and general applicability of normalization. It is recommended that aortic contractions be normalized to segment length and/or volume to reduce variability, enhance efficiency and to foster universal comparisons across myography platforms, laboratories and experimental settings. 


\section{Introduction}

Cardiovascular disease is the leading cause of death worldwide.

Atherosclerosis is the primary form of cardiovascular disease that increases the risk of myocardial infarction, stroke and peripheral arterial disease. An underlying mechanism of atherosclerosis involves a 'phenotypic switch' in vascular smooth muscle cells (VSMC) that transcriptionally moves VSMC from a contractile to a proliferative or "synthetic phenotype" (Li et al., 2013; Z. Zhang et al., 2012). Thus, (D. J. Conklin, Haberzettl, et al., 2009b) phenotypic change reflective of the underlying local cellular and biochemical alterations such as in atherosclerosis. Thus, laboratories around the world measure vascular contractile function in vitro wherein an isolated blood vessel, e.g., aorta, is contracted with an agonist, e.g., phenylephrine (PE), endothelin-1, U46,619 - a thromboxane $A_{2}$ analog, etc... and data reported (D. J. Conklin \& Boor, 1998; D. J. Conklin, Boyce, Trent, \& Boor, 2001b; D. J. Conklin, Haberzettl, et al., 2009b). The widespread use of in vitro vascular contractility assays is revealed in PubMed search using the terms "vascular" and "dysfunction," which netted 214,358 hits, indicating a sizeable research investment.

Although raw isometric data reflect vessel contractility, raw values (e.g., mg or $\mathrm{mN}$ tension)(Reynolds et al., 2012) cannot be compared across different laboratories due to obvious differences in the size of the blood vessel segment (e.g., length, diameter), species, animal size, age, diet, and disease state as well as the specific blood vessel or blood vessel location (e.g., thoracic vs abdominal aorta). Additionally, different platforms or myography systems (e.g., DMT) are used 
to assess isometric force production. So, even if two laboratories use the same system, the reporting of data can make it difficult to compare contractility due to dissimilar units ( $\mathrm{mg}$ vs $\mathrm{mN}$ ) or a different data normalization procedure, e.g., \% of control agonist such as \% contraction of high potassium solution)(Kim, Avila, \& Massett, 2016; Schepelmann et al., 2016) or differential normalization, e.g., mg tension/cross-sectional area; $\mathrm{mg}$ tension $/ \mathrm{mg}$ wet weight; $\mathrm{mN} / \mathrm{mm}$ length (D. J. Conklin \& Boor, 1998; D. J. Conklin et al., 2004; D. J. Conklin, Haberzettl, et al., 2009b). To address this obvious limitation and to foster more across-study and laboratory comparisons, aortic contractions were normalized by several easily measured parameters.

Several criteria need to be met to establish a universal normalization method. For example, the method needs to be accessible, and thus, it should not require costly equipment that may exclude users. It should be simple enough that all users can implement the method. Moreover, the method should be cost effective in that the time spent performing should be worth the effort. Because current laboratories already use some form of normalization (e.g., as "\% of control agonist”) (Kim et al., 2016), a new approach must be cost effective and enhance data quality over an existing practice. Finally, the method should foster data comparisons for both cross-laboratory fertilization and greater biological understanding. To this end, we offer a simple and cost-effective method for normalization of vascular contractility that requires minimal equipment and effort on the user's behalf, yet refines the measurement of aortic contraction to a degree wherein even modest changes in contractility (e.g., due to a treatment, aging, diet, 
etc...) are more readily discerned. Universally-normalized contractility data can be shared and compared by laboratories around the globe. Adoption of this approach may improve assessment of the biological actions of drugs, for example, and thus, minimize the overall use of resources.

\section{Materials and Methods}

\section{Rodents:}

Male C57BL/6 mice were purchased from The Jackson Laboratory (Bar Harbor, ME) or raised in house. Mice were fed normal chow (NC; $13 \% \mathrm{kcal}$ fat, Rodent Diet 5010, LabDiet; St. Louis, MO) or high fat (HFD; $42 \%$ kcal fat, TD.88137, Harlan Teklad, Madison, WI) diet from 8 to 20 weeks of age. Male Sprague-Dawley rats (wild type and low-density lipoprotein receptor deficient; LDLR-null) were obtained from SAGE (Sigma-Aldrich) and fed normal chow diet up to $1 \mathrm{yr}$ of age (Sithu et al., 2017). Rodents were treated according to APS's Guiding Principles in the Care and Use of Animals and all protocols were approved by University of Louisville IACUC. All rodents were anesthetized with sodium pentobarbital (i.p., $150 \mathrm{mg} / \mathrm{kg}$ ) and exsanguinated via right ventricle blood draw.

\section{Equipment:}

Digital handheld microscope (zORB, Carson, New York); scientific ruler (1 mm increments; Fine Science Tools); 10\% neutral buffered formalin solution (NBF; Sigma); Falcon® 12-well, clear, flat bottom plate (Corning Costar); ImageJ (NIH free imaging software); razor blades; dental wax (Z Pink); general surgical tools 
(scissors, forceps); microfuge tubes; organ bath myography system; and, microbalance.

Aorta Isolation and Organ Bath Systems:

Aortas were isolated via ventral thoracotomy and placed into cold phosphate buffered solution (PSS) containing (in $\mathrm{mM}$ ): $\mathrm{NaCl}, 130 ; \mathrm{KCl}, 4.7$; $\mathrm{MgSO}_{4} \cdot 7 \mathrm{H}_{2} \mathrm{O}, 1.17 ; \mathrm{KH}_{2} \mathrm{PO}_{4}, 1.18 ; \mathrm{NaHCO}_{3}, 14.9 ; \mathrm{CaCl}_{2}$, 2.0; glucose, 5.0; $\mathrm{pH}$ 7.4. Distal thoracic aorta (located $0.8-1 \mathrm{~cm}$ from aortic arch apex) segments were cut from each aorta (2-4 mm), cleaned of perivascular adipose tissue, and mounted in one of two systems: 1) horizontal pin (DMT, Ann Arbor, MI); or, 2) vertical wire system. In the horizontal pin system, a short, aortic segment $(\approx 2 \mathrm{~mm})$ was mounted on stainless steel pins in a $5-\mathrm{ml}$ heated $\left(37^{\circ} \mathrm{C}\right)$ organ bath. One pin was connected to an isometric strain gauge transducer, and the other was attached to a micrometer. Transducer signals were fed directly into a PC with LabChart software (v. 7 or 8 ; ADI). In the vertical wire system, an aortic segment (3-5 mm) was placed on wire hooks (100 $\mu \mathrm{m}$ dia. tungsten wire) with a hook connected to an isometric strain gauge transducer (Kent Scientific, Litchfield, CT) and the other attached to a fixed support glass rod in a $15-\mathrm{ml}$ heated $\left(37^{\circ} \mathrm{C}\right)$ organ bath. Transducer signals were fed into an 8-channel PowerLab A/D converter and recorded on a PC using LabChart software (v. 4.3.2; ADI). Prior to each use, each system was calibrated using standardized weights. In both systems, after 10 min without tension, aortic rings were equilibrated to $\approx 9.81 \mathrm{mN}(\approx 1 \mathrm{~g})$ loading tension over $30 \mathrm{~min}$ before agonist stimulation (D. J. Conklin, Haberzettl, Prough, \& Bhatnagar, 2009a). 
Statistics.

Values are expressed as mean \pm SD or \pm SE (where appropriate). Comparisons between 2 groups (e.g., horizontal vs vertical system; User 1 vs User 2; etc...) were done with a paired or unpaired $t$-test as appropriate. $\mathrm{P}<0.05$ was considered significant.

\section{Results and Discussion}

Acquisition of Aortic Segment Parameters:

Normalization of contractions was done using a simple, sequential, multistep process that is described pictorially in Fig. 1, and described here as a general protocol: 1) immediately formalin-fix a blood vessel segment; 2) obtain wet weight (to $0.1 \mathrm{mg}$; dab extra fluid on tissue paper); 3) image segment lengthwise with scientific ruler (Fig. 1a); 4) cut (with razor blade) and image (with handheld digital microscope $)$ a thin $(0.5 \mathrm{~mm})$ blood vessel cross-section $(x-s e c)$ with a ruler in the image (Fig. 1b); 5) using ImageJ, quantify segment length $(\mathrm{mm})$ and $\mathrm{x}-\mathrm{sec}$ area $\left(\mathrm{mm}^{2}\right)\left(\right.$ Fig. 1c); and, 6) calculate segment volume $\left(\mathrm{mm}^{3}\right)$ as the product of length

$(\mathrm{mm})$ and $\mathrm{x}-\mathrm{sec}$ area $\left(\mathrm{mm}^{2}\right)$, and the normalize isometric contractions by segment length $(\mathrm{mN} / \mathrm{mm}), x-s e c$ area $\left(\mathrm{mm}^{2}\right)$, and volume $\left(\mathrm{mN} / \mathrm{mm}^{3}\right)$. 
Figure 1. Images of method for aortic parameter data acquisition. a) Digital images of blood vessel segment length $(\mathrm{mm})$ acquired with digital minimicroscope camera. b) Digital image of blood vessel cross-section over-laid with digital outlines of outer and inner circumferences (NIH ImageJ). The outer area $(\mathrm{OA})$ minus the inner area $(\mathrm{IA})$ is the segment cross-sectional area $\left.\left(\mathrm{mm}^{2}\right) . \mathbf{c}\right)$ Calculation of blood vessel segment volume ( volume $=$ cross-section area * length $\left.=\mathrm{mm}^{3}\right)$. (Permission with S. Karger AG, Basel.) 

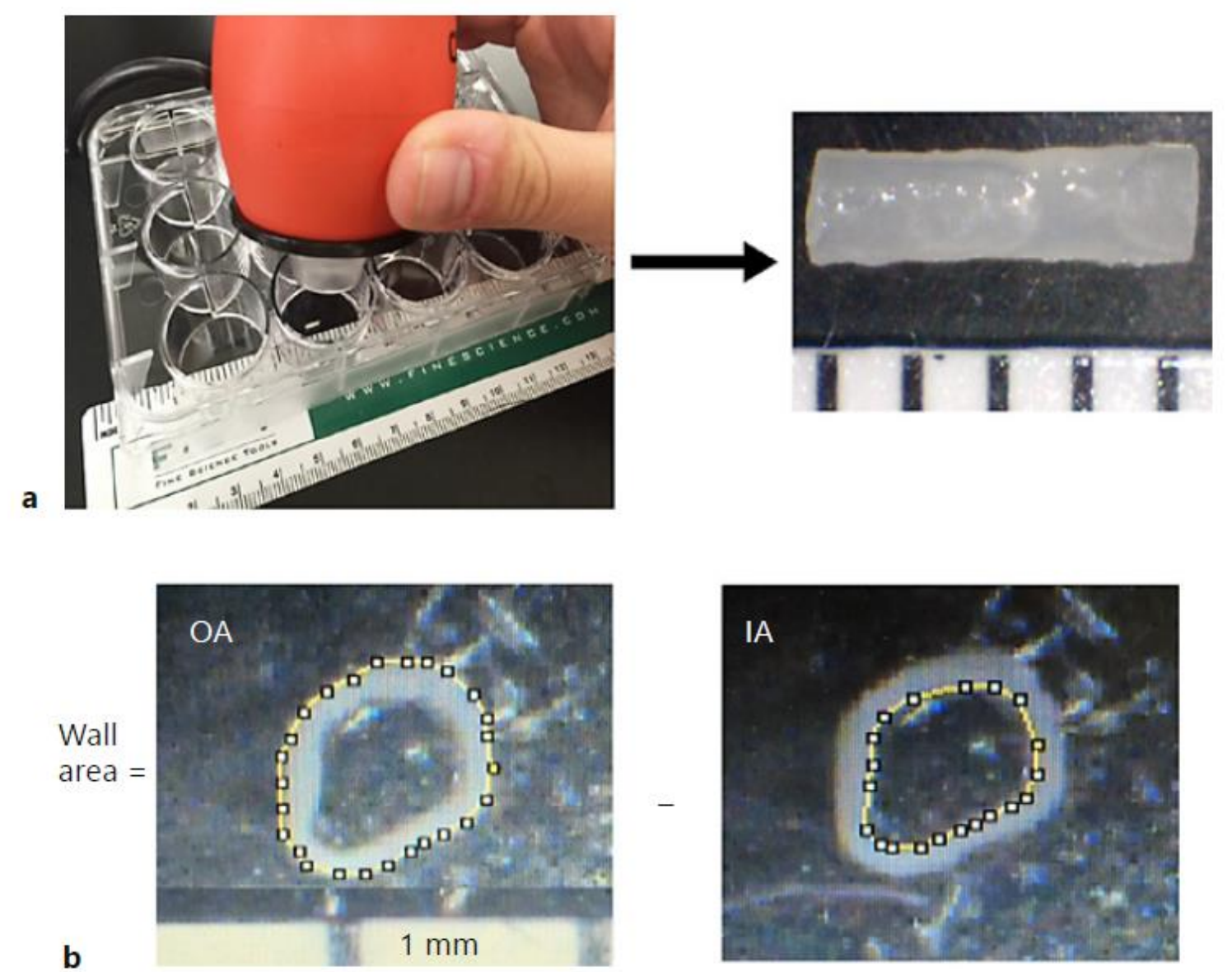

c Volume $=$ length $\times$ wall area $(O A-I A)=m m^{3}$ 


\section{Aortic Function:}

Aortic vascular contractile function was assessed using both a horizontal and a vertical isometric tension organ bath system as reported previously (D. J. Conklin, Haberzettl, et al., 2009a). To illustrate the utility of the normalization protocol, the distal thoracic aorta of a mouse was sectioned into 2 obvious, unequal lengths. A shorter piece $(1.5-2.5 \mathrm{~mm})$ was mounted in the horizontal pin system and a longer piece (3-4 mm) was mounted in the vertical system. Both segments were equilibrated similarly $(\approx 9.81 \mathrm{mN}$ tension). As intended in this 'proof of principle' example, the absolute (raw) tension of aortic contractions ( $\mathrm{mN}$ ) induced either by cumulative concentrations of PE (1 nM-10 $\mu \mathrm{M}$; Fig. $2 \mathbf{2 a})$ or by high potassium solution (100 mM; Fig. 2b) was significantly different between the two due to the intentional difference in blood vessel segment size $(\approx 2 x)$. 
Figure 2. Normalization of vascular contractility data. Contraction data (Raw, mN) and data normalized by aorta cross-sectional area $\left(\mathrm{mN} / \mathrm{mm}^{2}\right)$, length $(\mathrm{mN} / \mathrm{mm})$, and volume $\left(\mathrm{mN} / \mathrm{mm}^{3}\right)$ were plotted. To generate tension data, two aortic segments were exposed to contractile agents: a) phenylephrine (PE, $1 \mathrm{nM}-10 \mu \mathrm{M})$; and, b) high potassium (100 mM) buffer; in a horizontal pin (DMT; 1, filled symbols), or a vertical wire (2, open symbols) organ bath system. After experimentation, length and cross-sectional area of the blood vessel segments were measured and the segment volume calculated from these values (volume $=$ cross-section area * length; see Fig. 1). (Permission with S. Karger AG, Basel.) 

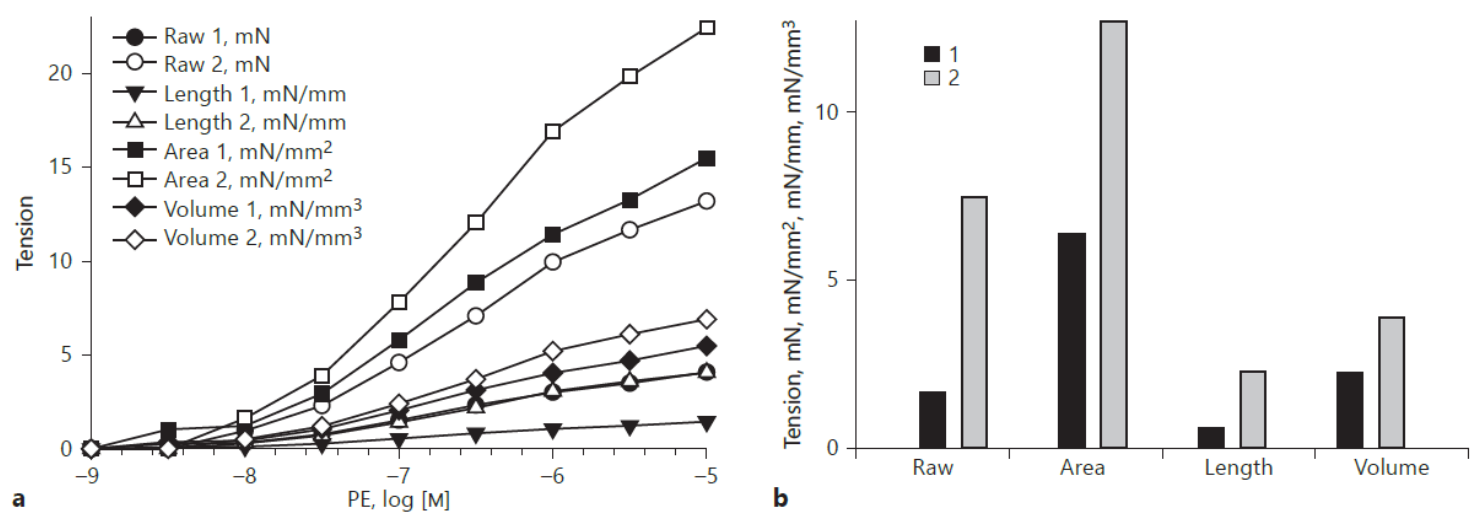
Normalization of Aortic Contraction:

After acquisition of aortic parameters, cumulative PE and high potassiuminduced contractions were normalized to $x-s e c$ area, length, and volume (Fig. 2). Normalization of raw tension by segment volume $\left(\mathrm{mN} / \mathrm{mm}^{3}\right)$ resulted in the most similar PE-induced tension curves (see curves of "Volume 1" and "Volume 2"; Fig. 2a). Whereas normalization of raw values to either $x-\sec$ area or length were beneficial in reducing the difference between "Raw 1" and "Raw 2" values, but neither was superior to normalization by volume. Notably, in a separate study, a group used a standardized mouse aorta segment length $(3 \mathrm{~mm})$ to normalize contractility and arrived at values similar to ours, thus, supporting the idea that normalized contractions even to one dimension (e.g., length; $\mathrm{mN} / \mathrm{mm}$ ) likely is better than no normalization (Fig. 2a) (Silva et al., 2016). Normalization efficacy was independent of the contractile agonist because a similar effect was observed when high potassium-induced maximal tension was normalized to $\mathrm{x}$-sec area, length and volume (Fig. 2b). As for PE, normalization to volume appeared to perform better than using either length or area alone.

In a second, real-world example, contractility was measured in aortas isolated from C57BL/6 mice fed either a normal chow $(13 \% \mathrm{kcal}$ fat) or high fat diet $(42 \% \mathrm{kcal}$ fat) for 12 weeks. As all aorta segments had similar lengths, $x$-sec areas, and thus, volumes (Table 1), direct comparison of raw contractility values revealed that 12 weeks of HFD appeared to decrease PE-induced contractions relative to aortas of NC-fed mice yet there were no statistically significant differences (Fig. 3a). To assess whether normalization would unmask differences in contractility, raw 
values were normalized by area (Fig. 3b), length (Fig. 3c) and volume (Fig. 3d). When normalized to aortic segment length or volume, contractions were statistically different between NC and HFD mice (Fig. 3c, d). Regression analyses revealed that aortic length (mm; Fig. 3e) and volume $\left(\mathrm{mm}^{3}\right.$; Fig. 3f) were correlated with contractility $(\mathrm{mN})$ more strongly in aorta of NC- than in HFD-fed mice. Moreover, volume appeared to perform better as a denominator than did length in both NC- and HFD-fed mice (Fig. 3e, f). Overall, these data indicate that normalization of aortic contractility enhanced detection of statistically significant changes induced by HFD (Fig. 3d). Another real world example was performed using aortas isolated from 1-year old Sprague-Dawley rats that were wild type or deficient of LDLR (LDLR-KO). As all aorta segments had similar lengths, $x$-sec areas, and thus, volumes (Table 2), direct comparison of raw contractility values revealed that genetic deletion of LDLR increased PE-induced contractions relative to aortas of WT rats (Fig. 4a). To assess whether normalization would alter the difference in contractility based on raw values, values were normalized by length (Fig. 4b), x-sec area (Fig. 4c) and volume (Fig. 4d). Normalization of raw contractility data to aortic segment length, area or volume only enhanced the statistically significant differences between WT and LDLR-KO rats (Fig. 4b, c, d). Overall, these data indicate that normalization of aortic contractility improves detection of statistically significant changes induced by genetic deletion of LDLR in rats. 
Table 1. Thoracic aorta segment parameters from C57BL/6 mice fed normal chow $(n=9)$ or high-fat diet $(n=10)$ for 12 weeks.

\begin{tabular}{|c|c|c|}
\hline \multirow{2}{*}{ Parameter } & \multicolumn{2}{|c|}{ Diet } \\
\cline { 2 - 3 } & Normal chow & High-fat diet \\
\hline Wall area, $\mathrm{mm}^{2}$ & $0.246 \pm 0.024$ & $0.225 \pm 0.020$ \\
\hline Length, $\mathrm{mm}$ & $3.96 \pm 0.56$ & $4.37 \pm 0.41$ \\
\hline Volume, $\mathrm{mm}^{3}$ & $0.976 \pm 0.178$ & $0.990 \pm 0.171$ \\
\hline
\end{tabular}

Values $=$ means \pm SE $; n=9,10$ mice, respectively. 
Figure 3. Normalization of vascular contractility of aortas from C57BL/6 mice fed normal chow (NC; $n=9$ mice) or high fat diet (HFD; $n=10$ mice) for 12 weeks. Phenylephrine (PE)-induced raw contraction data $(\mathbf{a}, \mathrm{mN})$ and raw data normalized by aorta length $(\mathbf{b}, \mathrm{mN} / \mathrm{mm}), \mathrm{x}-\mathrm{sec}$ area $\left(\mathbf{c}, \mathrm{mN} / \mathrm{mm}^{2}\right)$, and volume $\left(\mathbf{d}, \mathrm{mN} / \mathrm{mm}^{3}\right)$. Values $=$ mean \pm SE. $n=9$ or 10 aortas ( 1 aorta per mouse). ${ }^{*}, \mathrm{P}<0.05$ between NC and HFD. (Permission with S. Karger AG, Basel.) 

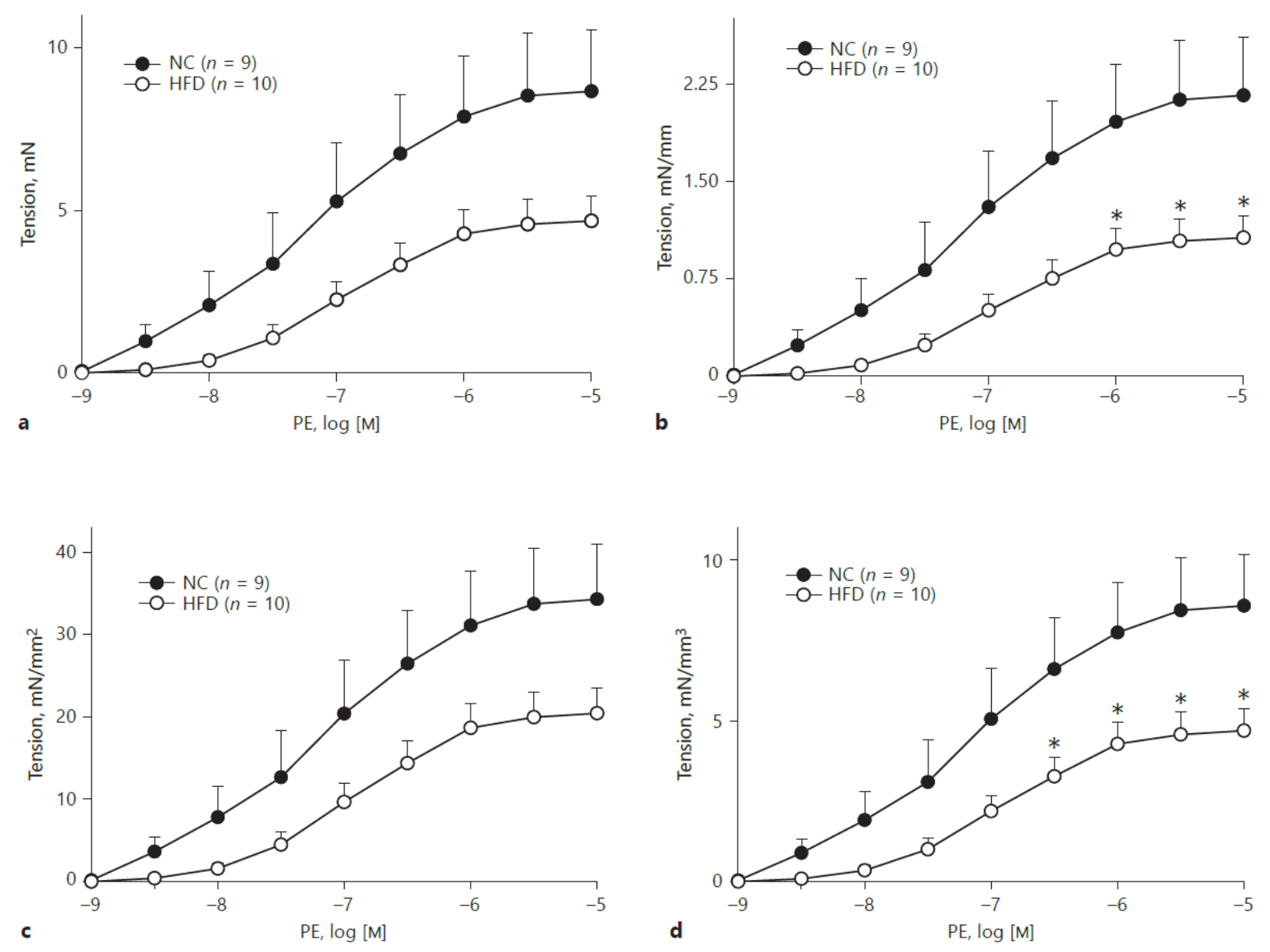
Table 2. Normalization of high potassium-induced $(100 \mathrm{mM})$ isometric contractions in thoracic aortas isolated from male mice fed either normal chow (NC) or high fat diet (HFD) for 12 weeks.

\begin{tabular}{|c|c|c|c|c|}
\hline Group & $\mathrm{mN}$ & $\mathrm{mN} / \mathrm{mm}$ & $\mathrm{mN} / \mathrm{mm}^{2}$ & $\mathrm{mN} / \mathrm{mm}^{3}$ \\
\hline $\mathrm{NC}$ & $8.26 \pm 1.03$ & $2.08 \pm 3.32$ & $33.16 \pm 3.32$ & $8.37 \pm 0.71$ \\
\hline HFD & $5.54 \pm 0.61$ & $1.26 \pm 0.13$ & $24.61 \pm 2.60$ & $5.66 \pm 0.57$ \\
\hline$p$ value & 0.0330 & 0.0047 & 0.0562 & 0.0079 \\
\hline
\end{tabular}

Value $=$ means \pm SE; $n=9,10$ mice, respectively; $p$ values shown are results of unpaired $t$ tests between NC and HFD groups. 
Table 3. Thoracic aorta segment parameters from wild-type (WT) and LDLR-null (LDLR-KO) 1-year-old Sprague-Dawley rats.

\begin{tabular}{|c|c|c|}
\hline \multirow{2}{*}{ Parameter } & \multicolumn{2}{|c|}{ Strain } \\
\cline { 2 - 3 } & WT & LDLR-KO \\
\hline Wall area, $\mathrm{mm} 2$ & $1.251 \pm 0.213$ & $1.168 \pm 0.224$ \\
\hline Length, $\mathrm{mm}$ & $3.07 \pm 0.49$ & $2.74 \pm 0.48$ \\
\hline Volume, $\mathrm{mm} 3$ & $3.90 \pm 1.055$ & $3.269 \pm 1.138$ \\
\hline
\end{tabular}

Values $=$ means \pm SE. $n=5,6$ rats, respectively. 
Table 4. Normalization of $\mathrm{PE}$-induced $(10 \mu \mathrm{M})$ isometric contractions in thoracic aortas isolated from wild-type (WT) and LDLR-null (LDLR-KO) 1-year-old Sprague-Dawley rats.

\begin{tabular}{|c|c|c|c|c|}
\hline Group & $\mathrm{mN}$ & $\mathrm{mN} / \mathrm{mm}$ & $\mathrm{mN} / \mathrm{mm}^{2}$ & $\mathrm{mN} / \mathrm{mm}^{3}$ \\
\hline NC & $15.10 \pm 2.55$ & $4.85 \pm 0.66$ & $12.09 \pm 1.59$ & $3.98 \pm 0.52$ \\
\hline HFD & $22.29 \pm 2.34$ & $8.36 \pm 1.09$ & $19.57 \pm 2.35$ & $7.50 \pm 1.27$ \\
\hline$p$ value & 0.0680 & 0.0283 & 0.0327 & 0.0413 \\
\hline
\end{tabular}

Value $=$ means \pm SE; $n=5,6$ rats, respectively; $p$ values shown are results of unpaired $t$ tests between WT and LDLR-KO groups. 
Figure 4. Normalization of vascular contractility of aortas from Sprague-Dawley wild type (WT; $n=5$ rats) and LDLR-KO rats ( $n=6$ rats) fed normal chow for 1 year. Phenylephrine (PE)-induced raw contraction data $(\mathbf{a}, \mathrm{mN})$ and raw data normalized by aorta length $(\mathbf{b}, \mathrm{mN} / \mathrm{mm}), \mathrm{x}-\mathrm{sec}$ area $\left(\mathbf{c}, \mathrm{mN} / \mathrm{mm}^{2}\right)$, and volume $\left(\mathbf{d}, \mathrm{mN} / \mathrm{mm}^{3}\right)$. Values $=$ mean \pm SE. $n=5$ blood vessels. ${ }^{*}, P<0.05$ between $\mathrm{WT}$ and LDLR-KO rats. (Permission with S. Karger AG, Basel.) 

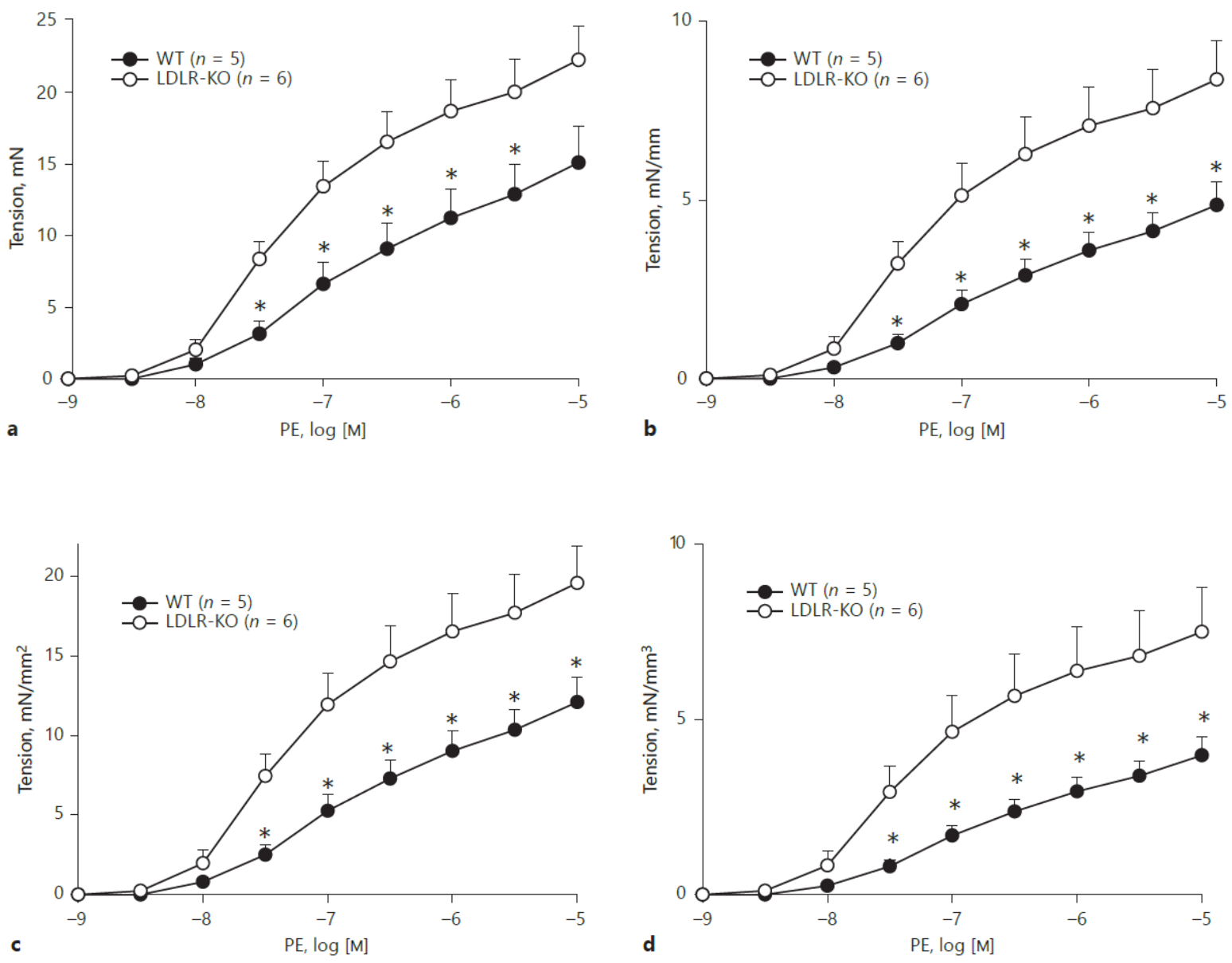


\section{Method Validation:}

The 'proof of principle' tests (i.e., platforms; NC vs HFD; WT vs LDLR-KO) support the general applicability of our normalization approach, so we also performed four validation tests. These were: 1) two different 'users' quantified the same ring images using ImageJ; 2) two different users quantified the same image multiple times (e.g., 10 times); 3) two different users imaged and then quantified multiple sections from a single aorta; and, 4) normalization was performed using segment volume calculated by 2 different methods: a) our image analysis approach; and, b) volume estimated from segment weight (in $\mathrm{mg}$ ) and density $\left(\mathrm{mg} / \mu \mathrm{l} ; \mathrm{mg} / \mathrm{mm}^{3}\right)$. 


\section{Reproducibility.}

Image analysis for length and $\mathrm{x}-\mathrm{sec}$ area proved highly reproducible (Fig. 5). It appeared that users vary in their first attempt to quantify length, but users rapidly adjusted and subsequently made consistent measurements of the same image. These data support the robust and cost-effective nature of our approach. Images were made with an inexpensive microscope camera $(<\$ 50)$ and were analyzed with free imaging software (NIH ImageJ). The approach was independent of the user because measurements made by 2 users of both area (Fig. 6a) and length (Fig. 6b) using the same 5 blood vessel segments were similar. Although noticeable divergence in volumes between users was present (Fig. 6c), both variability and directionality of values were preserved -- indicating both robustness and consistency. Our approach also compared favorably to using blood vessel volume estimated as the quotient of blood vessel mass and reported aortic density, i.e., $0.86 \mathrm{mg} / \mathrm{L}$ (Weind, Ellis, \& Boughner, 2002) (Fig. 6d). We think that there is inherently more difficulty in accurately measuring the mass of a wet blood vessel than our image-based approach. First, it should be recognized that a short, cleaned segment of mouse aorta weighs approximately $0.1-0.2 \mathrm{mg}$, and a highquality balance capable of such a measurement is costly. Second, much smaller

blood vessels, e.g., mesenteric, may be difficult to weigh accurately because of lumen fluid retention that is difficult to remove without rapidly "drying out" the blood vessel segment (Murphy, 1980). Thus, our low-cost approach is more practical and avoids these pitfalls. 
We also tested whether a single blood vessel segment would yield different $\mathrm{x}-\mathrm{sec}$ areas along its length and whether this was dependent on user evaluation. Although variation in $\mathrm{x}-\mathrm{sec}$ area was apparent, it appeared less dependent on user and more on image quality and segment location (Fig. 7). These data further emphasize that specific user-based analysis of an image is a less important determinant of $x-s e c$ area than is the image quality and blood vessel location (e.g., thoracic aorta tapers proximal to distal). Of course, quality control of image analysis needs to be established in each laboratory adopting this approach. We purposefully used a low cost, highly portable 'hobby' or classroom-style digital camera/microscope to emphasize that even rudimentary optics are sufficient to perform blood vessel analysis yet practice is required.

Resiliency to Prolonged Formalin Fixation.

Formalin fixation for 1 week (Fig. 8a), 1 month (Fig. 8b) and up to 2 months (Fig. 8c) had little effect on quantified aortic segment volume, so it is feasible to formalin fix a blood vessel and still obtain reliable and robust measurements for normalization long after contractility experiments were performed. Additionally, fixed blood vessel segments can be processed, embedded, sectioned and stained for more refined morphometry of the blood vessel wall (D. J. Conklin, Kong, \& Committee, 2015). 
Figure 5. Reproducibility of aorta parameter data acquisition: intra-assay variation. The same images of a blood vessel lengthwise and in cross-section (ring) were repeatedly examined for length and cross-sectional area (x-sec area), respectively, as described above using Image $\mathrm{J}$ a total of 10 times by two different observers. Blood vessel volume $\left(\mathrm{mm}^{3}\right)$ was calculated by multiplying length $(\mathrm{mm}) \times \mathrm{x}$-sec area $\left(\mathrm{mm}^{2}\right)$. Values $=$ mean \pm SD. $($ Permission with S. Karger AG, Basel. $)$ 


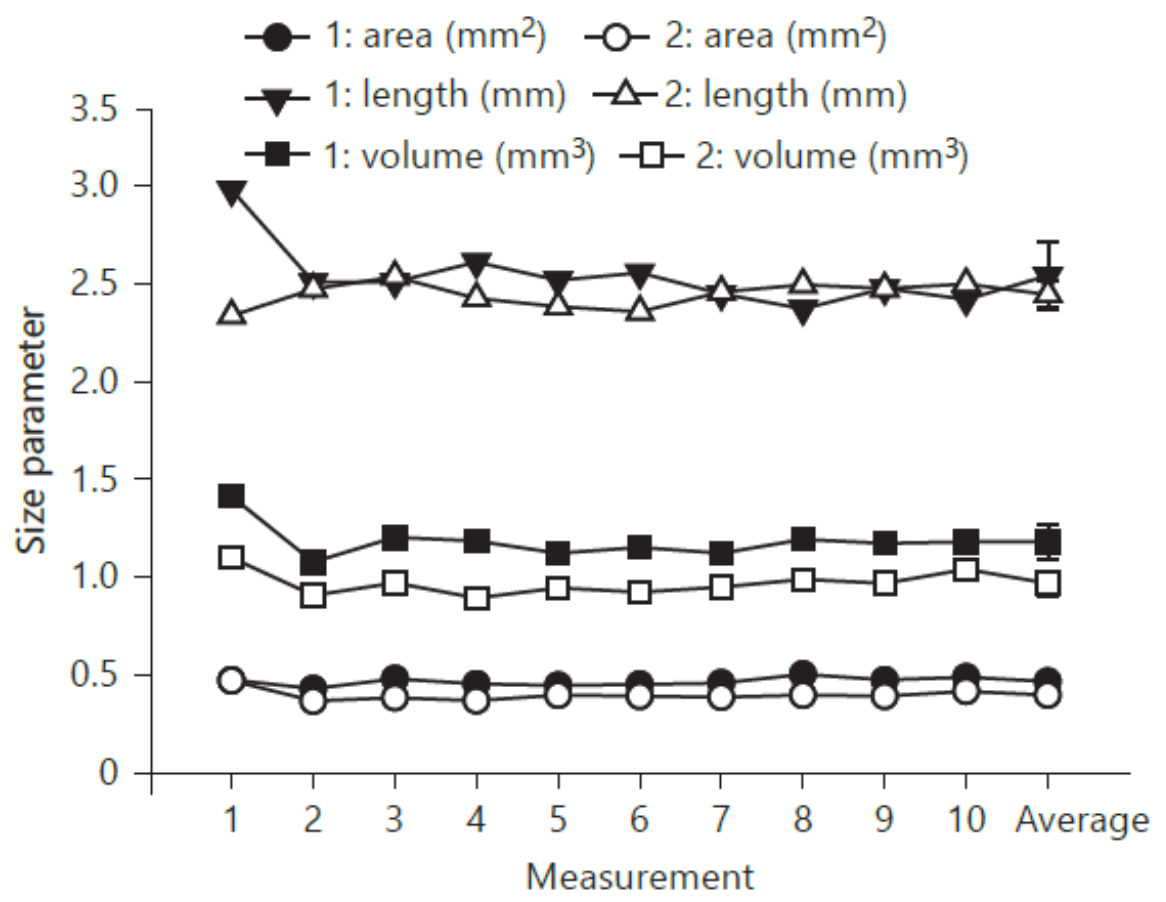


Figure 6. Reproducibility of aorta parameter data acquisition: inter-assay variation. a) Aorta cross sectional area $\left(\mathrm{mm}^{2}\right)$ measured in five separate aortas independently by two users (User 1; User 2). b) Aorta segment length (mm) measured independently by two different users in five separate aortas. c) Volume $\left(\mathrm{mm}^{3}\right)$ calculated five times by two users. d) Aortic volume calculated from image analysis compared with volume derived from segment weight and aorta density $(0.86 \mathrm{mg} / \mathrm{L})$. Values $=$ mean $\pm \mathrm{SD}$. (Permission with S. Karger AG, Basel. $)$ 

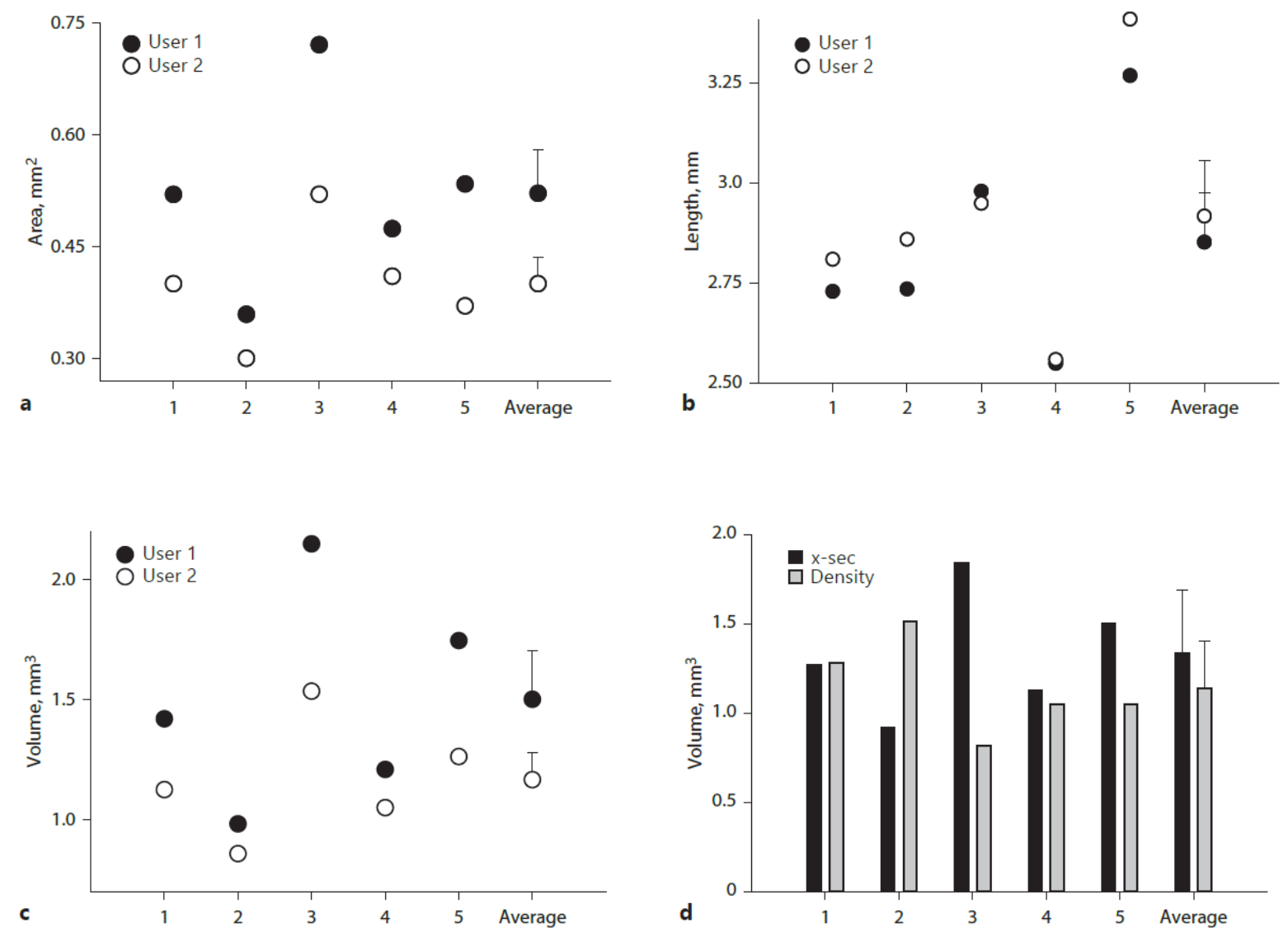
Figure 7. Intra-aortic variation in cross-sectional area. A single thoracic aorta segment was cut into five cross-sections ("donut") and each cross-section was imaged and the area measured by two independent users (User 1; User 2). Values $=$ mean \pm SD. (Permission with S. Karger AG, Basel.) 


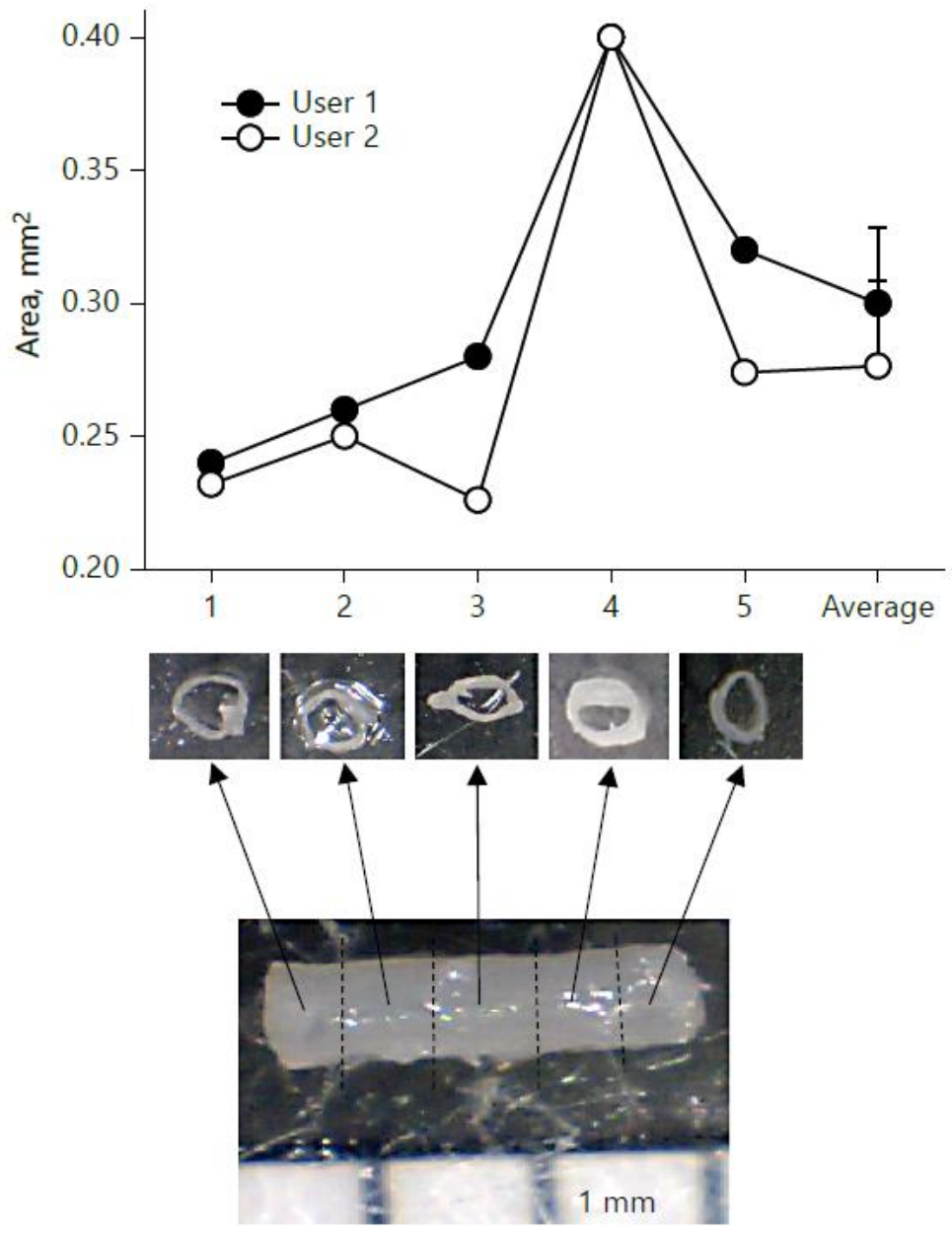


Figure 8. Resiliency of aorta parameter data acquisition over time. Comparison of aorta volumes measured after formalin fixation of the blood vessel. A) Aorta volume measured 1 week after formalin fixation. B) Aorta volume measured 1 month after formalin fixation. C) Aorta volume measured 2 months after formalin fixation. Values $=$ mean \pm SD. (Permission with S. Karger AG, Basel.) 

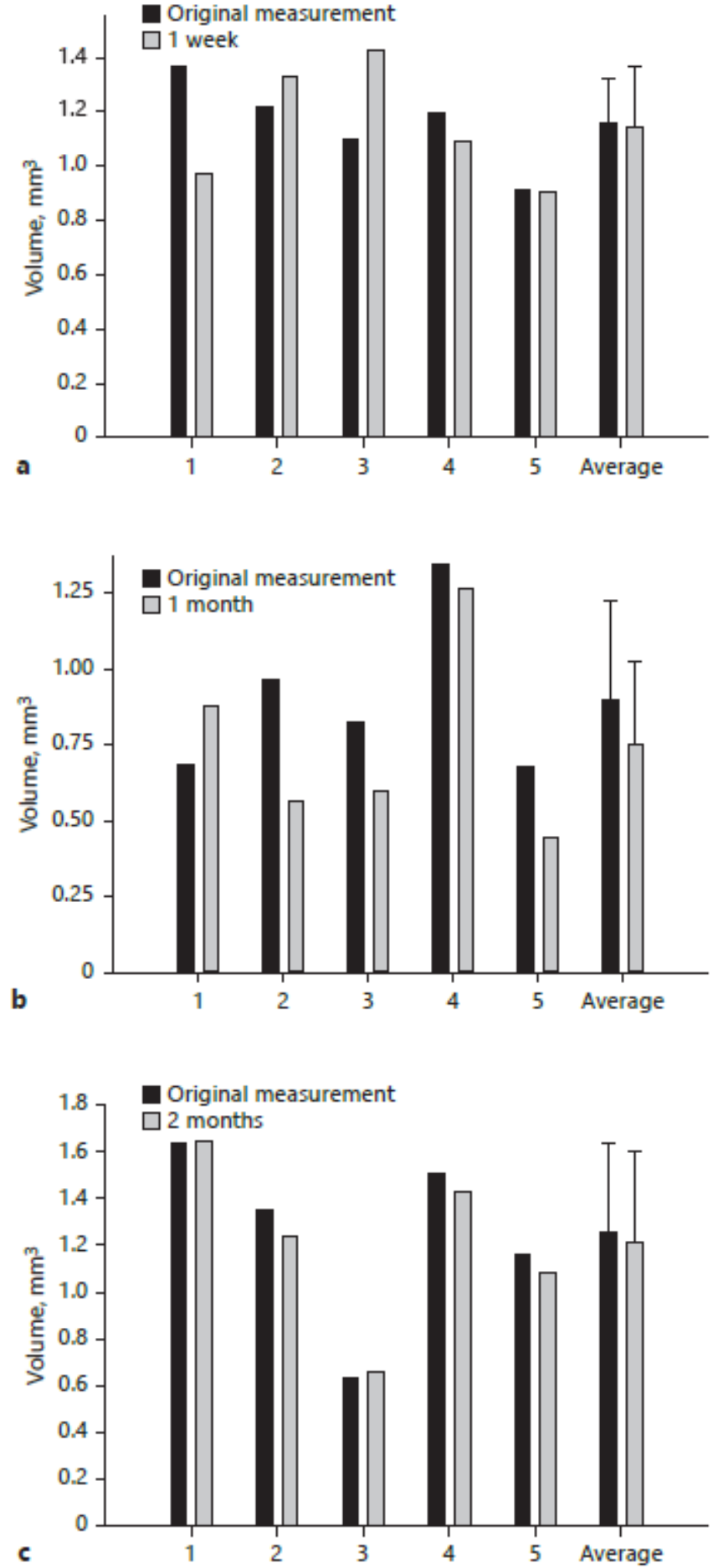


\section{Major Advantages and Limitations:}

The current study describes a novel method for normalization of vascular contractility in vitro, and highlights 4 major advantages: 1) the method is simple and low cost, and thus, accessible world-wide; 2) the method is validated and is reproducible across different platforms and users; 3 ) the method is resilient to the chronic storage of blood vessels in formalin; and, 4) if adopted, normalized vascular contraction data with a 'universal unit' (either $\mathrm{mN} / \mathrm{mm}$ or $\mathrm{mN} / \mathrm{mm}^{3}$ ) will allow for more across study and across laboratory comparisons, and thus, more efficient use of research resources.

There are several caveats to this method. This method does not define the optimal state of contractility or the mechanisms of change in contractility. It is expected that laboratory conditions likely will vary in many ways that meaningfully contribute to real differences in contractility (e.g., buffer composition, loading tension, temperature, inhibitors, denuding, etc...), but "all things being equal," this approach provides a common denominator, which currently is not used by most laboratories. As indicated above, the method reduces variability in raw values by correcting for differences that arise as a function of blood vessel segment size. Blood vessel segments differ in size simply because two different laboratories use different platforms. An obvious limitation is that we only tested the protocol using aortas from mice and rats, however, the method will likely work in other blood vessels because there is an inherit reliance of vascular contraction on smooth muscle that makes up the bulk of the media in aorta and other arteries as well. In any case, we predict that normalization (with segment length and/or volume) is 
superior to using only raw contraction values. In our real-world example, we detect significant changes in aortic contractility with HFD feeding (vs NC) only when we normalized contractions (see Fig. 3). This outcome has value because importantly it reduces the overall cost of a study by reducing the number of animals needed in a study. Finally, our approach is validated to be robust, consistent, reproducible and resilient. It is recommended that isometric vascular contractions be normalized to either blood vessel segment length $(\mathrm{mN} / \mathrm{mm})$ or volume $\left(\mathrm{mN} / \mathrm{mm}^{3}\right)$ to foster both improved quantitative assessment of contractility changes and across study comparisons. 
CHAPTER III

\section{FORMALDEHYDE INDUCES MESENTERIC ARTERY RELAXATION VIA A SENSITIVE TRANSIENT RECEPTOR POTENTIAL ANKYRIN-1 (TRPA1) AND ENDOTHELIUM-DEPENDENT MECHANISM}

Introduction Formaldehyde (FA), the smallest aldehyde, is generated endogenously, and is widespread in the environment in foods, beverages and as a gas phase product of incomplete combustion. Methods and Results The main metabolite of FA, formate, was increased significantly in murine urine $(\approx 3 x)$ after overnight feeding. Because feeding increases mesenteric blood flow, we explored the direct effects of FA in isolated murine superior mesenteric artery (SMA). Over the concentration range of $30-1,200 \mu \mathrm{M}$, FA strongly and reversibly relaxed contractions of SMA induced by three different agonists: phenylephrine (PE), thromboxane $A_{2}$ analog $(\mathrm{U} 46,619)$ and high potassium (high $\mathrm{K}^{+}, 60 \mathrm{mM} \mathrm{K} \mathrm{K}^{+}$). Formate (to $1.5 \mathrm{mM}$ ) did not induce relaxation. FA $(>1,500 \mu \mathrm{M})$ irreversibly depressed vascular function in SMA indicating vasotoxicity. The sensitivity $\left(\mathrm{EC}_{50}\right)$ but not the efficacy (\% relaxation) of FA-induced relaxations was dependent on blood vessel type (SMA $<$ aorta) and contractile agonist $\left(\mathrm{PE}, \mathrm{EC}_{50}=52 \pm 14 \mu \mathrm{M}\right.$; U46,619, $\left.\mathrm{EC}_{50}=514 \pm 129 \mu \mathrm{M} ; 60 \mathrm{~K}, \mathrm{EC}_{50}=1,093 \pm 87 \mu \mathrm{M}\right)$. The most sensitive 
component of FA vasorelaxation was within physiological levels $(30-150 \mu \mathrm{M})$ and was inhibited significantly by: 1) mechanically-impaired endothelium; 2) N $\omega$-NitroL-arginine methyl ester hydrochloride (L-NAME); 3) transient receptor potential ankyrin-1 (TRPA1) antagonist (A967079); 4) guanylyl cyclase (GC) inhibitor (ODQ); and, 5) $\mathrm{K}^{+}$channel inhibitor $\left(\mathrm{BaCl}_{2}\right)$. A similar mechanism of SMA vasorelaxation was stimulated by the TRPA1 agonist cinnamaldehyde. Positive TRPA1 immunofluorescent staining and gene-specific sequence were present in SMA but not in aorta. Conclusion These data indicate FA, but not formate, robustly relaxes SMA via a sensitive TRPA1- and endothelium-dependent mechanism that is absent in aorta. Thus, as FA levels increase with feeding, FA likely contributes to the physiological reflex of post-prandial hyperemia via SMA vasodilatation.

\section{Introduction}

Formaldehyde (FA) is the smallest aldehyde and the concentration of FA in the blood ranges from 0.0127 to $2.28 \mu \mathrm{g} / \mathrm{mL}$ (1.9 to $5.4 \mathrm{mg} / \mathrm{L}$; or $63-179$ $\mu \mathrm{M})($ Kleinnijenhuis, Staal, Duistermaat, Engel, \& Woutersen, 2013; Y. S. Wang et al., 2011). FA is in food and beverages (likely the largest quantitative source of exogenous FA) and is metabolically-derived (D.J. Conklin, P. Haberzettl, J. Lee, \& S. Srivastava, 2011). Exogenous FA is a volatile indoor and outdoor pollutant emitting from building materials (e.g., plywood, glues, carpet, drapery), and present in cosmetics, vehicle exhaust, photochemical smog and tobacco-derived aerosols (D. J. Conklin, P. Haberzettl, J. Lee, \& S. Srivastava, 2011; Ogunwale et al., 2017). Endogenous FA is generated by breakdown of L-methionine, histamine, 
methanol and methylamine, and may contribute to biological methylation of folic acid (Yu \& Zuo, 1996). Thus, blood levels of FA are in constant flux due to rapid changes in exposure, absorption, distribution, metabolism (to formate and $\mathrm{CO}_{2}$ ) and excretion.

Although direct effects of FA on cardiovascular targets are described, the role of FA in cardiovascular physiology is not well explored. For example, intraarterial or intravenous FA reduces heart rate (bradycardia), decreases arterial pressure (hypotension) and causes arrhythmias in animals (Strubelt, Brasch, Pentz, \& Younes, 1990; Tani \& Horiguchi, 1990; Tani, Kogi, \& Horiguchi, 1986; Tani, Satoh, \& Horiguchi, 1978), but these are considered toxicological effects due to high doses administered directly into the blood. Because FA is toxic in cultured endothelial cells (inhibition of mitosis, increased apoptosis), vascular smooth muscle cells and cardiomyocytes (D. J. Conklin, Langford, \& Boor, 1998), it is, thus, speculated that endogenously-generated FA induces endothelium dysfunction by which it accelerates diabetic complications such as atherosclerosis (Deng \& Yu, 1999). However, FA induces a concentration-dependent relaxation in isolated blood vessels (D. J. Conklin et al., 2004; Tani, 1981; Q. Zhang et al., 2018) yet the relevance of this effect in vascular physiology is unclear.

Despite myriad sources of FA and its provoked cardiovascular effects, few studies address the vascular effects of physiological levels of FA. Formate is a stable metabolite of FA generated from a 2-step enzymatic process involving glutathione-dependent formaldehyde dehydrogenase (FALDH2) hemi-thioacetal formation and aldehyde dehydrogenase $2(\mathrm{ALDH} 2)$ mediated oxidation (Conklin et 
al., 2006). In the current study, we measured urine levels of formate, the primary metabolite of FA, to estimate changes in endogenous FA levels. As murine urine levels of formate increased $\approx 3$ times overnight (with feeding) compared with levels after daytime fast, we think feeding increased endogenous FA levels (directly or indirectly). As such, the gastrointestinal vasculature represents a likely target of changes in FA levels following feeding when reflexively blood flow increases in the gut (Takagi, Naruse, \& Shionoya, 1988). Thus, to address potential FA-induced vascular effects, isolated superior mesenteric artery (SMA) and aorta were exposed to FA across a physiologic to toxic range $(30-1,500 \mu \mathrm{M})$. Although an FAinduced vasorelaxation was present in both blood vessels, the most sensitive relaxation was present in SMA indicating that physiologic levels of FA likely stimulate dilation in SMA before aorta. Thus, we identified the mechanisms of the FA-induced relaxation (30-150 $\mu \mathrm{M})$ as dependent on: a functional endothelium, TRPA1 activation, NO formation, guanylyl cyclase activation, and potassium channels. Formate, though, had no effect in blood vessels up to $1,500 \mu \mathrm{M}$. These data collectively support the idea that feeding increases FA levels, and that FA (and not formate) likely contributes to increased gastrointestinal blood flow of feeding (i.e., postprandial hyperemia) through a sensitive, robust and reversible TRPA1- and endothelium-dependent mechanism.

\section{Material and Methods}

Chemicals and Solutions. 
Reagent grade chemicals were purchased from Sigma-Aldrich or other commercial sources as indicated: A967079 (AdooQ); acetylcholine chloride (ACh); 4-aminopyridine (4-AP); barium chloride (BaCl2); cinnamaldehyde ( \%); formalin (formaldehyde, $37 \%$ and methanol, 10-15\%); formate; 1h-[1,2,4]oxadiazolo[4,3a]quinoxalin-1-one (ODQ); N $\omega$-nitro-L-arginine methyl ester hydrochloride ( $L$ NAME); L-phenylephrine hydrochloride (PE); sodium nitroprusside (SNP); tetraethylammonium chloride (TEA); and, U46,619 (thromboxane A2 analog). Krebs physiological salt solution (PSS) for SMA was (in $\mathrm{mM}$ ): $\mathrm{NaCl}, 119 ; \mathrm{KCl}, 4.7$; $\mathrm{CaCl}$, 2.5; $\mathrm{MgCl} 2$, 1.2; $\mathrm{KH} 2 \mathrm{PO} 4,1.2 ; \mathrm{NaHCO}$, 24; glucose, 7.0; pH 7.4. Krebs PSS for aorta was (in mM): $\mathrm{NaCl}, 118 ; \mathrm{KCl}, 4.7 ; \mathrm{CaCl} 2,2.5 ; \mathrm{KH} 2 \mathrm{PO} 4,1.2 ; \mathrm{MgSO}$, 1.2; NaHCO3, 12.5; and, glucose, 5.5; pH 7.4. High K+ PSS (60K; $60 \mathrm{mM})$ substituted equimolar $\mathrm{K}+$ for $\mathrm{Na}+$.

\section{Animals.}

Wild type C57BL/6J mice from in house breeding pairs or purchased (The Jackson Laboratory, Bar Harbor, ME) (12-20 weeks old; 25-35g) were used in these studies. Mice were treated according to American Physiological Society Guiding Principles in the Care and Use of Animals, and all protocols were approved by University of Louisville Institutional Animal Care and Use Committee. Mice were housed under pathogen-free conditions in the University of Louisville vivarium under controlled temperature and $12 \mathrm{~h}$ light:12h dark cycle. Mice were provided a standard chow diet (Rodent Diet 5010, 4.5\% fat by weight, LabDiet; St. Louis, MO).

Urine Collection and Formate Measurement. 
Urine samples were collected, centrifuged (2,500 RPM, $\left.10 \mathrm{~min}, 4{ }^{\circ} \mathrm{C}\right)$, decanted, and stored at $-80^{\circ} \mathrm{C}$ until analysis (D. J. Conklin et al., 2017). In brief, each mouse was briefly exposed to D-glucose/saccharin solution (w/v; $3.0 \% / 0.125 \%$; Sigma) immediately prior to a $6 \mathrm{~h}$ fast after which each was placed singly in a metabolic cage (Harvard Apparatus) with glucose/saccharin solution in drinking water without food to collect urine (in $4^{\circ} \mathrm{C}$ water-jacketed organ baths) for 3h. In total, mice were fasted for 9-h. After fasted urine collection, mice were provided food along with glucose/saccharin solution for an overnight urine collection $(\mathrm{O} / \mathrm{N})$. Urine samples were collected, centrifuged, decanted, and stored at $-80^{\circ} \mathrm{C}$ (D. J. Conklin et al., 2017).

The primary urinary metabolite of FA is formate and it was measured using GC-MS as described previously (Kage, Kudo, Ikeda, \& Ikeda, 2004; Lamarre et al., 2014) with minor modifications. Briefly, urine $(50 \mu \mathrm{L})$ was mixed with sodium phosphate $(20 \mu \mathrm{L} 0.5 \mathrm{M}, \mathrm{pH} 8.0)$ containing internal standard $\left({ }^{13} \mathrm{C}\right.$-formate, $\left.2.3 \mathrm{mM}\right)$. Samples were incubated with PFBBr $(130 \mu \mathrm{L}, 0.1 \mathrm{M})$ for 15 min at $60^{\circ} \mathrm{C}$, extracted with hexane (330 $\mu \mathrm{L})$, and analyzed by GC-MS in electron ionization (EI) mode. Six-point calibration curve was used to calculate the concentration of formate. Results were corrected for the natural abundance of ${ }^{13} \mathrm{C}$ isotope. Formate level was normalized to urinary creatinine $(\mathrm{mg})$ to account for urine dilution.

Isolated Superior Mesenteric Artery (SMA) and Aorta.

Mice were anesthetized with sodium pentobarbital $(0.1 \mathrm{ml}, 150 \mathrm{mg} / \mathrm{kg}$, i.p.), and SMA and aorta were removed via mid-ventral thoracotomy. Thoracic aorta rings (3-4 mm) were hung on stainless steel hooks in 15-ml water-jacketed organ 
baths and SMA rings $(2 \mathrm{~mm})$ were hung on tungsten wire $(75 \mu \mathrm{m}$ dia.) in 5 -ml heated organ baths (MultiWire Myograph System 620M, DMT, Denmark) in PSS bubbled with $95 \% \mathrm{O} 2: 5 \% \mathrm{CO} 2$ at $37{ }^{\circ} \mathrm{C}$. After 10 min without tension, aorta rings were equilibrated to $\approx 1 \mathrm{~g}$ loading tension over $30 \mathrm{~min}$ and SMA rings were equilibrated to $\approx 0.25 \mathrm{~g}$ loading tension over $1 \mathrm{~h}$. All rings were stimulated with high $\mathrm{K}+$ to test for viability, washed 3 times with PSS over $30 \mathrm{~min}$, and re-equilibrated to appropriate resting tension.

FA-Induced Relaxation in Isolated Superior Mesenteric Artery (SMA) and Aorta:

Formaldehyde $(30-1,500 \mu \mathrm{M})$ was added to organ baths containing either aorta or SMAs pre-contracted with 1 of 3 contractile agonists: phenylephrine (PE, $10 \mu \mathrm{M}), \mathrm{U} 46,619(0.1 \mu \mathrm{M})$, or high $\mathrm{K}^{+}(60 \mathrm{mM})$. The efficacy of FA-induced relaxation was calculated as the \% reduction in agonist-induced contraction. The sensitivity of FA-induced relaxation was calculated as the effective concentration producing $50 \%$ response $\left(\mathrm{EC}_{50}\right)$, i.e., cumulative concentration responses were normalized to $100 \%$ with interpolation of $\mathrm{EC}_{50}$. To assess whether time of day affected SMA response to FA, mice were euthanized both in day and in night cycle. Sensitivity to a known TRPA1 agonist, cinnamaldehyde (1-100 $\mu \mathrm{M})$, were conducted, and because cinnamaldehyde is immiscible in aqueous solution, dilutions were vigorously vortexed immediately prior to addition to organ baths.

Effects of Formate (FA metabolite) and Methanol: FA is oxidized to formate (primary urinary metabolite), and thus, we tested whether formate could induce vasorelaxation or vascular toxicity $(50$ to $1,500 \mu \mathrm{M})$ in PE pre-contracted aorta and 
SMA. Because formalin (a source of FA) contains $10-15 \%$ methanol (as a stabilizer), we also tested whether formalin or methanol alone (to $0.001 \%$, equivalent to amount present in $1,200 \mu \mathrm{M}$ formalin) contributed, in part, to FAinduced relaxation.

Role of Endothelium, Nitric Oxide Synthase (NOS), and cGMP:

To evaluate the role of NOS, L-NAME $(0.1 \mathrm{mM})$ was added prior (15 min) to addition of PE $(10 \mu \mathrm{M}), \mathrm{U} 46,619(0.1 \mu \mathrm{M})$ or High $\mathrm{K}^{+}(60 \mathrm{mM})$. Pre-contracted SMA rings were then relaxed with cumulative concentrations of FA $(50,150$, and $300 \mu \mathrm{M})$. The role of the endothelium was assessed as vasorelaxation to increasing FA concentrations $(50,150,300$, and $600 \mu \mathrm{M})$ in aorta and in SMA with intact and injured endothelium. The endothelium was mechanically-injured by air perfusion, and effective impairment was confirmed by near complete abolition (>95\%) of ACh-induced dilation of PE pre-contracted blood vessels. To assess whether cGMP was involved in FA-induced vasorelaxation, isolated blood vessels were pre-incubated with $\mathrm{ODQ}(3 \mu \mathrm{M})$ to inhibit guanylyl cyclase $(\mathrm{GC})$ and subsequent formation of cGMP (Jiang, Wan, Wang, \& Wu, 2015).

\section{Role of TRPA1 Channel:}

The role of the TRPA1 channel was assessed by addition of TRPA1 antagonist (A967079, 1 MM; 10 min; Cat. \#: A12353, AdooQ, Invine, CA) or DMSO (vehicle) in PE-precontracted aorta and SMA followed by cumulative addition of FA $(30-300 \mu M)$.

Role of $\mathrm{K}^{+}$Channels: 
The role of $\mathrm{K}^{+}$-channels in $\mathrm{FA}$-induced responses were interrogated using $\mathrm{K}_{\mathrm{IR}}$ channel inhibitor $\left(\mathrm{BaCl}_{2}, 1 \mathrm{mM}\right)$ and the non-specific $\mathrm{Kv}$ channel inhibitor (tetraethylammonium, TEA, $10 \mathrm{mM}$ ).

Role of nicotine:

Because most cigarette and e-cigarette contains nicotine, we tested whether nicotine would affect FA-induced relaxation in PE-precontracted SMA.

Histology and TRPA1 Immunofluorescence.

Thin sections $(4 \mu \mathrm{m})$ of formalin-fixed, paraffin-embedded SMA and dorsal root ganglia (DRG; positive control) of WT mice were stained with H\&E and TRPA1 antibody (1:200; Alomone Labs, Israel; Cat. \#: ACC-037)(N. Green, Weech, \& Walters, 2005). Images of SMA sections were made using a digital Spot camera mounted on an Olympus microscope. Immunofluorescence microscopy was performed with rabbit polyclonal antibody against TRPA1 without or with a blocking peptide of the TRPA1 antibody and Alexa Fluor 647 goat anti-rabbit secondary antibody (1:400 dilution; Invitrogen; Cat. \#: 21244). To label endothelial cells, sections were co-stained with isolectin GS-IB4 Alexa Fluor 594 conjugate (1:200 dilution; Invitrogen; Cat. \#: 121413). Briefly, the slides were deparaffinized, and heat-mediated antigen retrieval was performed in $10 \mathrm{mM}$ citrate buffer $(\mathrm{pH} 6.0)$ for 20 min at $95^{\circ} \mathrm{C}$. The slides were cooled to room temperature and then incubated with blocking buffer (10\% goat serum, $1 \%$ BSA, $0.025 \%$ triton X-100 in 1 X TBS) for $2 \mathrm{~h}$ at RT and then incubated with either TRPA antibody in blocking buffer without and with blocking peptide against TRPA1 (the TRPA1 antibody and the 
blocking peptide at $1: 1 \mathrm{ratio} ; \mu \mathrm{g} / \mu \mathrm{g}$ ) was mixed and pre-incubated for $1 \mathrm{~h}$ at $\mathrm{RT}$ before adding on top of the tissue sections) in blocking buffer overnight at $4^{\circ} \mathrm{C}$. The slides were incubated with anti-rabbit Alexa Fluor 647 secondary antibody in the dark for $1 \mathrm{~h}$ at RT. The slides were washed $3 \times 5$ min with 1 X TBST after each step. Tissue sections incubated with secondary antibody only served as a negative control. Slides were covered with DAPI containing Slow Fade ${ }^{\circledR}$ Gold anti-fade reagent (Invitrogen; Cat. \#: S36938) and fluorescence was visualized on a Nikon eclipse Ti fluorescence Microscope using NIS-Elements (Nikon; Japan) at 200X. A DAPI filter was used for nuclear staining and a Cy5 Red filter for TRPA1 staining. TRPA1 qRT-PCR.

Total RNA was isolated from approximately $10 \mathrm{mg}$ of SMA (8 arteries pooled) and DRG (positive control) from WT mice with Trizol (Invitrogen) and RNeasy MiniKit (QIAGEN) according to manufacturer's instructions. RNase-free DNase (QIAGEN; Cat. \# 79254) was used to remove any contaminating DNA. RNA was eluted from RNeasy mini-columns into $30 \mu \mathrm{L}$ of RNAse-free water and the concentration measured using NANODROP 2000C (Thermo Scientific). Total cDNA was synthesized using iScript cDNA synthesis Kit (BIO-RAD; Cat. \# 1708891) according to manufacturer's instructions. Briefly, $15 \mu \mathrm{L}$ denatured RNA (250 ng), $4 \mu \mathrm{L} 5 \mathrm{X}$ iScript reaction mix and $1 \mu \mathrm{L}$ of iScript reverse transcriptase were mixed (total volume, $20 \mu \mathrm{L}$ ) and cDNA synthesis was carried out in a BioRad mycycler Thermocycler using the following conditions: $42{ }^{\circ} \mathrm{C}$ for $60 \mathrm{~min}$ and $94^{\circ} \mathrm{C}$ for 5 min. The resultant cDNA was target amplified using TRPA1 specific primers. (Forward 5'-GAT GCC TTC AGC ACC CCA TTG- 3'; Reverse 5'-CAC 
TTT GCG TAA GTA CCA GAG TGG- 3'). Real-time quantitative PCR was done using a murine specific TaqMan gene expression assays. Briefly, the following were added to each well of a 384-well plate sequentially: $5.0 \mu \mathrm{L}$ of $2 \mathrm{X}$ TaqMan Fast Universal PCR Master Mix (Cat.no. 4440040), $2 \mu \mathrm{L}$ undiluted cDNA from target specific PCR and $3 \mu \mathrm{L}$ of murine primers for mTrpa1, (Mm01227447_m1, Cat. \# 4351372) or $\beta$-actin (Mm00607939_m1, Applied Biosystems, Cat. \# 4331182) for TaqMan assays (total $=10 \mu \mathrm{L}$ final volume), and then subjected to qRT-PCR using standard protocols on an Applied Biosystems 7900 HT Real-Time PCR system. For each RNA sample, the cDNAs were run in duplicate for each assay set in the same plate along with $\beta$-actin. The expression level of TRPA1 in both SMA and DRG are expressed as ct values.

DNA Sequencing and Agarose Gel Electrophoresis.

PCR products from TRPA1 TaqMan assay were run on $1.5 \%$ agarose gel and stained with ethidium bromide to visualize the expected $101 \mathrm{bp}$ band in WT SMA and DRG. Also, the PCR product from Target specific TRPA1 amplification (309 bp) of SMA and DRG were sequenced on Illumina NextSeq 500 by the Center for Genetic and Molecular Medicine (CGEMM University of Louisville) sequencing service core.

Statistical Analyses.

Data are expressed as means $\pm \mathrm{SE}$. When comparing two groups, a $t$-test was used (paired or unpaired as appropriate), and statistical significance was assumed where $\mathrm{P}<0.05$ (SigmaPlot, ver. 11). 


\section{Results}

Formate: the primary FA metabolite is increased with feeding.

Formate is the primary metabolite of FA, and formate in the urine is a consequence of endogenous FA metabolism, so we measured urinary formate by GC-MS. We found that feeding significantly increased the urinary levels of formate indicating a major source of endogenous FA is either directly from food or related to feeding, e.g., metabolism of food constituents, intermediary metabolism, etc... In male $\mathrm{C} 57 \mathrm{BL} / 6 \mathrm{~J}$ mice, a daytime fast $(9 \mathrm{~h})$ resulted in a basal level of urinary formate that was tripled with overnight feeding (Table 5). A similar pattern occurred in female C57BL/6J mice (data not shown). Thus, we infer that feeding increased FA levels that subsequently increased formate excretion in urine.

\section{FA-induced relaxation.}

FA (from $30 \mu \mathrm{M}$ to $1.2 \mathrm{mM}$ ) induced a robust, repeatable and concentration- and agonist-dependent relaxation in isolated SMA and aorta of both male and female

mice (Fig. 9 A-D; Table 6). Regardless of agonist (PE, U46,619, high $\mathrm{K}^{+}$), FA relaxed agonist-induced tension by $>90 \%$ in SMA and aorta (Table 7 ), yet when added to an uncontracted vessel, FA did not affect basal tone (myogenic tone; data not shown). FA-induced relaxation up to $1.2 \mathrm{mM}$ (SMA) and $1.5 \mathrm{mM}$ (aorta) did not alter subsequent agonist-induced contractions or ACh-induced (endothelium-dependent) relaxation indicating no overt vasotoxicity. Irreversible toxicity was, however, detected following exposure to high levels of FA ( $>1.2 \mathrm{mM}$ in SMA; $>1.5 \mathrm{mM}$ in aorta) with vasotoxicity defined as near loss of contractility 
even after multiple bath changes (e.g., 3 washouts, $<10 \%$ tension production of first agonist-induced contraction; data not shown).

\section{FA: formate and methanol.}

Because FA is metabolized to formate, it is possible that vascular effects of FA (and its precursors) are the result of FA metabolism to formate. To address this possibility, formate was cumulatively added up to $2.1 \mathrm{mM}$ in PE pre-contracted SMA. Formate induced a weak relaxation indicating that FA-induced relaxation was not a result of formate production (Table 7). Formalin, a mixture of FA (37\%) and methanol (10-15\% as stabilizer), induces TRPA1-dependent vasodilation and pain (McNamara et al., 2007), yet it is unknown if this is due to either FA or methanol (or both). Because FA also is a product of methanol metabolism, we tested whether methanol alone (at levels comparable with those present in formalin) had any effect in PE pre-contracted SMA. Methanol also induced a weak, modestly concentration-dependent relaxation in SMA. Thus, neither formate nor methanol appears responsible for the efficacy of FA- or formalin-induced relaxations, respectively. These data also implicate real time metabolism of FA (to formate) as a relevant vaso-regulatory step because it converts an active agent (FA) into an inactive metabolite (formate). Thus, alterations in FA metabolism in the vascular wall may be a targetable process to promote or diminish feedinginduce hyperemia.

Role of endothelium, nitric oxide synthase (NOS), and GC in FA-induced vasorelaxation. 
To investigate the mechanism of the most sensitive component of the FAinduced relaxation, we used PE-precontracted SMA (PE<U46,619<60K). Because the SMA has a robust ACh-induced, endothelium-dependent relaxation that was impaired significantly by air perfusion (Fig. 10), we tested for the role of endothelium in FA-induced relaxation. Air perfusion significantly shifted FAinduced relaxation rightward in SMA (Fig. 11 A, B). Similarly, the sensitivity $\left(E_{50}\right)$ but not the efficacy of FA-induced vasorelaxation in PE-pre-contracted SMA was affected by L-NAME $(100 \mu \mathrm{M})$ (Fig. 11 C, D). However, L-NAME had no effect on sensitivity or efficacy of FA-induced relaxation in either U46,619 (514 \pm 129 vs $569 \pm 98 \mu \mathrm{M})$ or High- $\mathrm{K}^{+}(1,093 \pm 87$ vs $984 \pm 92 \mu \mathrm{M})$ pre-contracted SMA (Fig. $10 \mathrm{C}$, D). The rightward shift due to L-NAME indicated a role of NO (e.g., eNOS) as part of the most sensitive component of the FA-induced relaxation in PE-precontracted SMA. Because NO stimulates guanylyl cyclase (GC) to generate cGMP in smooth muscle cells, we tested ODQ, an irreversible inhibitor of GC, and ODQ also rightward shifted the FA-induced relaxation (Fig. 11 E, F). The endothelium contributes to overall contractility in SMA (Fig.10 B, E). Collectively, these data show that the most sensitive component of the FA-induced relaxation is dependent on a functional endothelium, NOS and an NO-mediated activation of GC.

Role of TRPA1 channel.

Because formalin-induced pain is associated with activation of TRPA1 channel as well as an increase in blood flow (McNamara et al., 2007), we hypothesized that the TRPA1 channel may contribute to the FA-induced, endothelium-dependent relaxation in SMA. In support, the TRPA1 antagonist 
A967079 $(1 \mu \mathrm{M})$ significantly inhibited the most sensitive portion of the FA-induced relaxation $(30 \mu \mathrm{M})$ in PE-contracted SMA (Fig. 12 A, B). In paraffin-embedded, thin cut sections of mouse SMA, TRPA1 (green) was co-localized with isolectin (red) in endothelium (Fig. 12 C). More modest yet specific TRPA1 staining was present in SMA media and adventitia as well. Positive and selective TRPA1 staining was confirmed by inclusion of a blocking peptide and by using dorsal root ganglion (DRG; Fig. 12 D) as a positive control. A PCR product specific to TRPA1 exons 23-25 mRNA (Fig. 12 Ei) matched both the predicted fragment size (101 bp; Fig. 12 Eii) and by gene sequence (Fig. 12 Eiii) -- confirming TRPA1 presence in SMA. These image and molecular data support that TRPA1 contributed to the FAinduced, endothelium-dependent relaxation in SMA.

Cinnamaldehyde is a known TRPA1 agonist, and, unsurprisingly, it induced a concentration- and TRPA1-dependent relaxation in SMA (Fig. 13 A). This relaxation, like FA, this sensitive cinnamaldehyde-induced relaxation in SMA was dependent on a functional endothelium (Fig. 13 B), TRPA1 (Fig. 13 C), NOS (Fig. 13 D) and GC (Fig. 13 E).

Role of $\mathrm{K}^{+-}$-channels.

In SMA pre-contracted with High $\mathrm{K}^{+}(60 \mathrm{mM}), \mathrm{FA}$ induced an insensitive (rightward-shifted) relaxation (compared with both PE and U46,619). From these data, we surmised that alteration of smooth muscle membrane potential, likely due to $\mathrm{K}^{+}$channel activation, contributed to FA-induced vasorelaxation in PEcontracted SMA. In confirmation, $\mathrm{BaCl}_{2}(1 \mathrm{mM})$, which blocks inward rectifier $\mathrm{K}^{+}$ channels, significantly inhibited FA-induced relaxation in PE-contracted SMA (Fig. 
14 A, B) and also in aorta (Fig. 14 C). However, we observed no effect of the nonselective $\mathrm{K}^{+}$channel inhibitor, TEA (tetraethylammonium, $10 \mathrm{mM}$ ), on FA-induced relaxation in SMA (Tables 7,8 ). Collectively, these results indicate that the activation of $\mathrm{K}_{\mathrm{IR}}$ channels but not of other $\mathrm{K}^{+}$channels was involved in $\mathrm{FA}$-induced vasorelaxation in PE-precontracted SMA. The endothelium also contributed to FAinduced relaxation in aorta (Fig. 14 D)

Nicotine and FA:

In tobacco users, FA may be present in blood simultaneously with nicotine, so we also tested whether nicotine altered FA-induced relaxation in SMA. Nicotine alone $(1 \mu \mathrm{M})$ had no effect in PE-contracted SMA, and also had no effect on subsequent FA-induced vasorelaxation. 
Fig. 9. Formaldehyde (FA) induced relaxation in superior mesenteric artery (SMA). A) Representative traces of concentration-dependent relaxation induced by cumulative addition of FA $(50-1,200 \mu \mathrm{M})$ in isolated SMA contracted by one of three different contractile agonists: phenylephrine (PE); thromboxane $A_{2}$ analog (U46,619); or, high potassium (60K). B) Summary data of the efficacy (\% relaxation) of FA-induced relaxation in isolated pre-contracted SMA. Summary data of the efficacy (\% relaxation; C) and of the sensitivity (D) of FA-induced relaxation in isolated PE-precontracted SMA from male and female mice isolated either during day time or night time. Values are means \pm SE of 3-10 preparations. 
A.

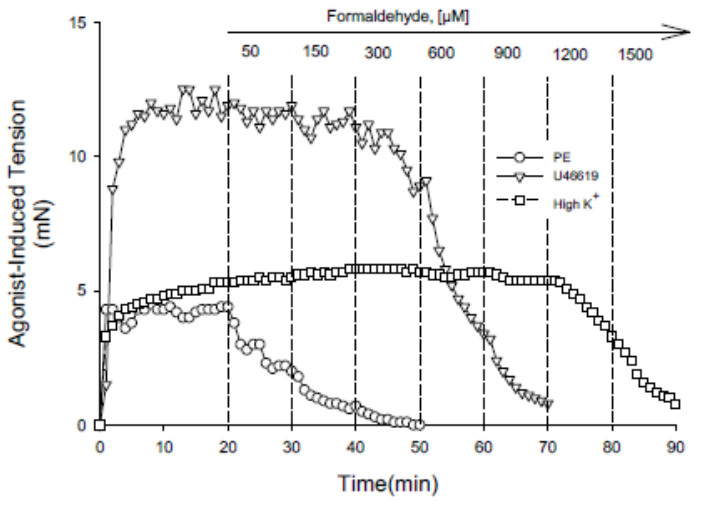

C.

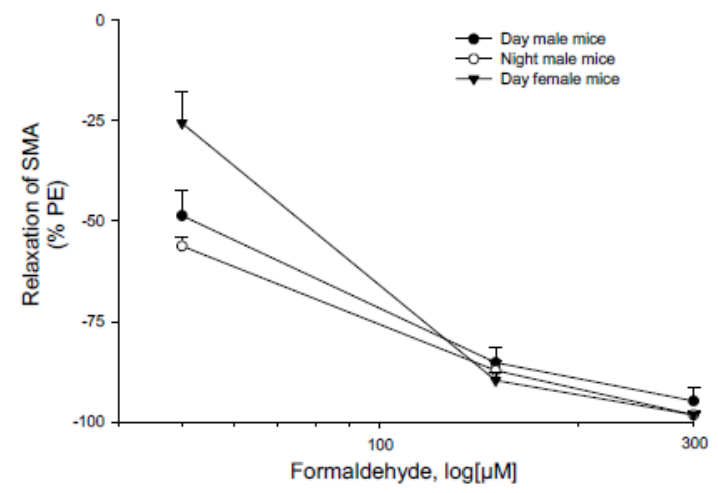

B.

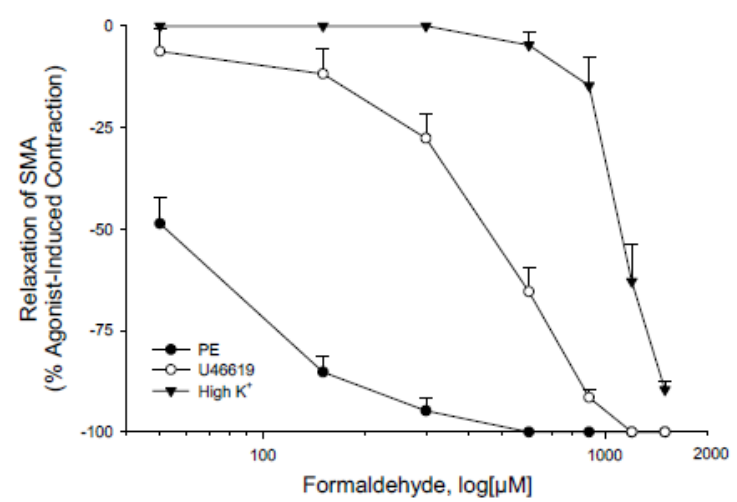

D.

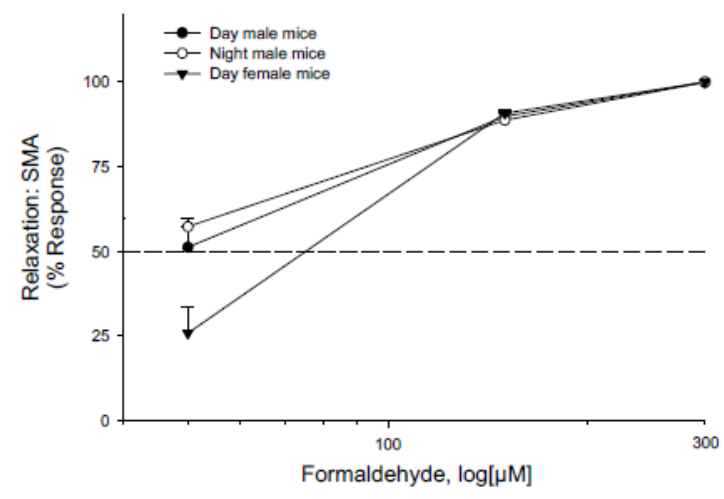


Table 5. Levels of urinary formate following either a daytime fast or overnight feeding in male C57BL/6J mice.

\begin{tabular}{|c|c|c|}
\hline & \multicolumn{2}{|c|}{ Condition } \\
\hline Urine Metabolite & $\begin{array}{c}\text { Daytime Fast } \\
\text { (9h, no food, 3h water) }\end{array}$ & $\begin{array}{c}\text { Overnight Feeding } \\
\text { (16h, food \& water) }\end{array}$ \\
\hline Formate $(\mu \mathrm{g} / \mathrm{ml})$ & $0.72 \pm 0.03$ & $2.73 \pm 0.95^{*}$ \\
\hline Creatinine $(\mathrm{mg} / \mathrm{dl})$ & $6.88 \pm 0.32$ & $7.23 \pm 0.66$ \\
\hline $\begin{array}{c}\text { Formate }(\mu \mathrm{g} / \mathrm{mg} \\
\text { creatinine }\end{array}$ & $10.54 \pm 0.74$ & $36.82 \pm 10.30^{*}$ \\
\hline
\end{tabular}

Values $=$ means $\pm S E . N=3-5$ per group. ${ }^{*}, \mathrm{P}<0.05$ vs fasting. 
Table 6. The effective concentration inducing $50 \%$ relaxation $\left(\mathrm{EC}_{50}\right.$; in $\left.\mu \mathrm{M}\right)$ of formaldehyde-induced vasorelaxation in isolated murine superior mesenteric artery (SMA; male and female; day and night) and thoracic aorta precontracted with different agonists.

\begin{tabular}{|c|c|c|c|}
\hline \multirow{2}{*}{$\begin{array}{c}\text { Blood Vessel } \\
\text { (sex, time of day) }\end{array}$} & \multicolumn{3}{|c|}{ EC 50 by precontraction agonist } \\
\hline & PE & U46,619 & High $\mathrm{K}^{+}$ \\
\hline SMA (male, day) & $53 \pm 3$ & $514 \pm 129^{*}$ & $1,093 \pm 87^{\star}$ \\
\hline SMA (male, night) & $43 \pm 2$ & -- & -- \\
\hline SMA (female, day) & $77 \pm 9$ & -- & -- \\
\hline Aorta (male, day) & $261 \pm 22^{*}$ & -- & -- \\
\hline
\end{tabular}

Values = means \pm SE. Abbr.: PE, phenylephrine, [10 $\mu \mathrm{M}]$; U46,619, thromboxane $\mathrm{A}_{2}$ analog, [0.1 $\left.\mu \mathrm{M}\right]$; High $\mathrm{K}^{+}$, high potassium solution, [60 $\left.\mathrm{mM} \mathrm{K}^{+}\right]$; --, did not perform experiment. $\mathrm{N}=3-10$ per group. ${ }^{*}, \mathrm{P}<0.05$ vs EC50 of "SMA (male, day)" PE agonist (i.e., baseline control). 
Table 7. Efficacy of formaldehyde and associated compounds in relaxation of PEprecontracted superior mesenteric artery (SMA) and aorta without and with selective treatments (inhibitors).

\begin{tabular}{|c|c|c|c|c|c|}
\hline \multirow{2}{*}{ Compounds } & \multicolumn{2}{|c|}{$[\mathrm{mM}]$} & \multirow{2}{*}{ Treatment } & \multicolumn{2}{c|}{ \% Relaxation } \\
\cline { 2 - 3 } \cline { 5 - 6 } & SMA & Aorta & & SMA & Aorta \\
\hline Formaldehyde & 0.3 & 0.6 & - & $-94.3 \pm 1.3$ & $-99.6 \pm 1.2$ \\
\hline Formate & 2.1 & - & - & $-25.7 \pm 6.7$ & - \\
\hline Methanol & 2.1 & - & - & $-34.1 \pm 11.7$ & - \\
\hline Formaldehyde & 0.3 & 0.6 & ED & $-90.4 \pm 2.3$ & $-94.0 \pm 2.6$ \\
\hline Formaldehyde & 0.6 & - & $+\mathrm{LNAME}$ & $-96.7 \pm 2.2$ & - \\
\hline Formaldehyde & 0.3 & 0.6 & $+\mathrm{A} 967079$ & $-96.4 \pm 1.1$ & $-100.7 \pm 1.2$ \\
\hline Formaldehyde & 0.6 & - & $+\mathrm{ODQ}$ & $-99.7 \pm 0.5$ & - \\
\hline Formaldehyde & 0.6 & 0.6 & $+\mathrm{BaCl}_{2}$ & $-99.5 \pm 0.5$ & $-81.7 \pm 3.7$ \\
\hline Formaldehyde & 0.2 & 0.6 & $+\mathrm{TEA}$ & $-90 \pm 1.8$ & $-94.4 \pm 1.3$ \\
\hline
\end{tabular}

Values $=$ means \pm SE. Abbr.: PE, phenylephrine, $10 \mu \mathrm{M}$; ED, endothelial dysfunction induced by mechanical air perfusion; LNAME, $\mathrm{N}^{\omega}$-nitro-L-arginine methyl ester hydrochloride, $100 \mu \mathrm{M}$; A967079, TRPA1 antagonist, $3 \mu \mathrm{M}$; ODQ, 1h[1,2,4]oxadiazolo[4,3-a]quinoxalin-1-one, $\mathrm{BaCl}_{2}$, barium chloride, $1 \mathrm{mM}$; TEA, tetraethylammonium. $\mathrm{N}=3-6$ per group. 
Fig. 10. Regulators of agonist-induced tension in superior mesenteric artery (SMA). A) Phenylephrine (PE), U46,619- and 60K-induced tension $(\mathrm{mN})$ in SMA in the absence and presence of LNAME. B) FA-induced relaxations (\%) in PE, U46,619and 60K-precontracted SMA in the absence and presence of LNAME. C) FAinduced relaxations (converted to $100 \%$ ) in PE, U46,619- and 60K-precontracted SMA in the absence and presence of LNAME. D) Representative tracings of PEinduced tension and ACh-induced relaxation in SMA with intact and mechanicallyimpaired endothelium (ED). E) Summary data of PE-induced tension in SMA in the absence and presence of LNAME, $\mathrm{BaCl}_{2}, \mathrm{ED}$, and $\mathrm{ODQ}$. Values are means $\pm \mathrm{SE}$ of 3-4 preparations. *, $P<0.05$ vs Control. 
A.

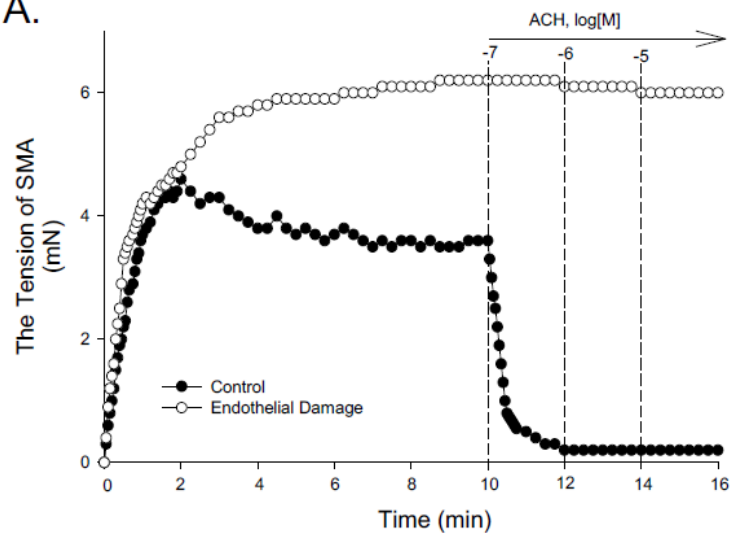

C.

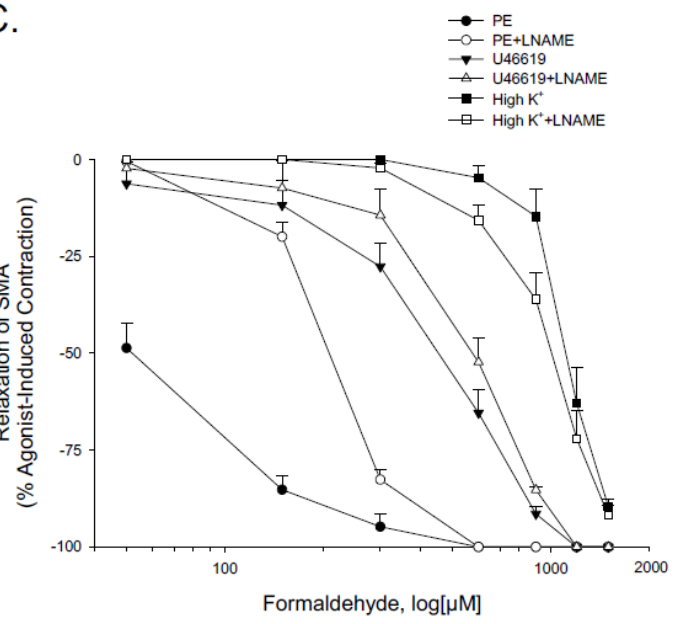

E.

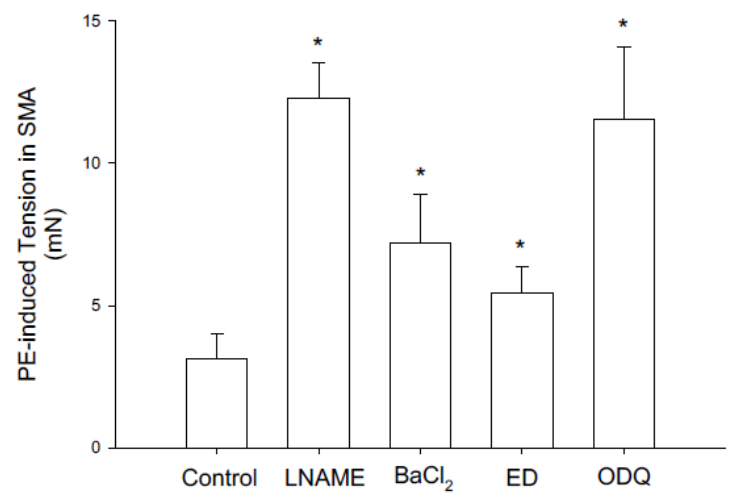

B.

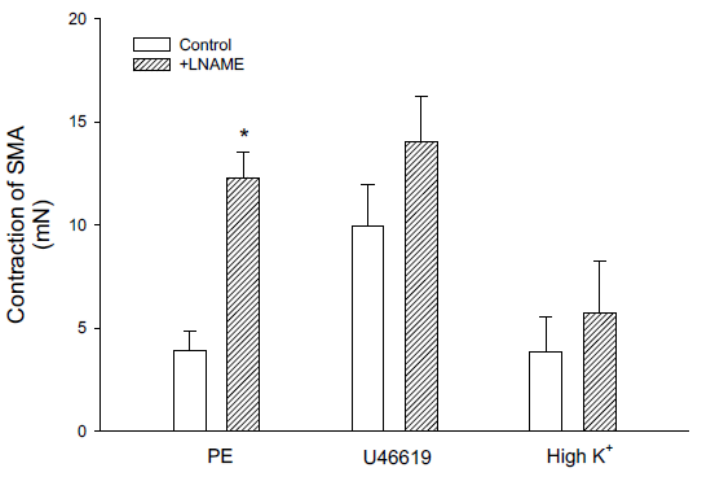

D.
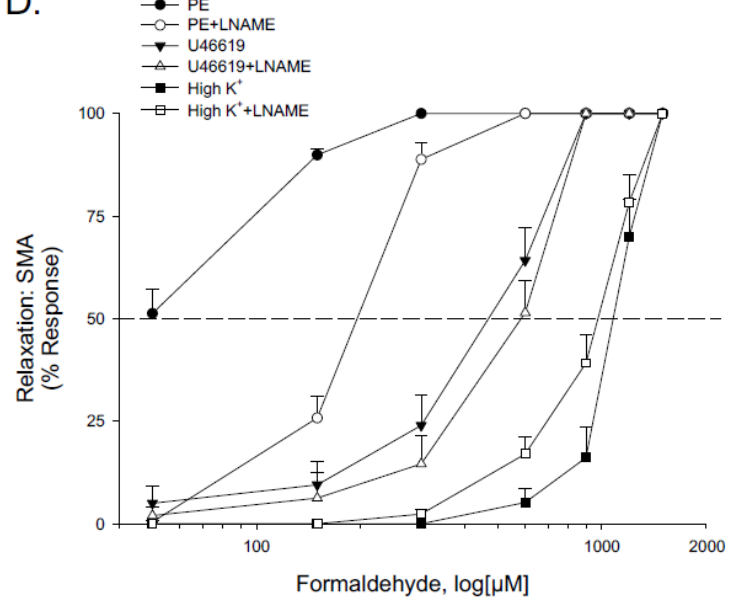
Fig. 11. Role of the endothelium and nitric oxide (NO) in the most sensitive component of formaldehyde-induced relaxation of phenylephrine (PE) precontracted superior mesenteric artery (SMA). A) Representative traces of FAstimulated relaxation of PE-induced contraction without and with endothelium impairment due to $5 \mathrm{~min}$ of air perfusion. B) Summary data of FA-induced relaxation in isolated SMA pre-contracted with PE in the presence and absence of functional endothelium. C) Representative traces of FA-stimulated relaxation of PE-induced contraction in the presence and absence of LNAME. D) Summary data of FA-induced relaxation in isolated SMA pre-contracted with PE in the absence and presence of LNAME. E) Representative traces of FA-stimulated relaxation of PE-induced contraction in the presence and absence of the guanylyl cyclase (GC) antagonist, ODQ $(3 \mu \mathrm{M})$, added to bath after PE-induced contraction plateaued and prior to cumulative addition of FA. F) Summary data of FA-induced relaxation in isolated SMA pre-contracted with PE in the absence and presence of ODQ. Values are means \pm SE of 3-4 preparations. ${ }^{*}, P<0.05$ vs Control. 

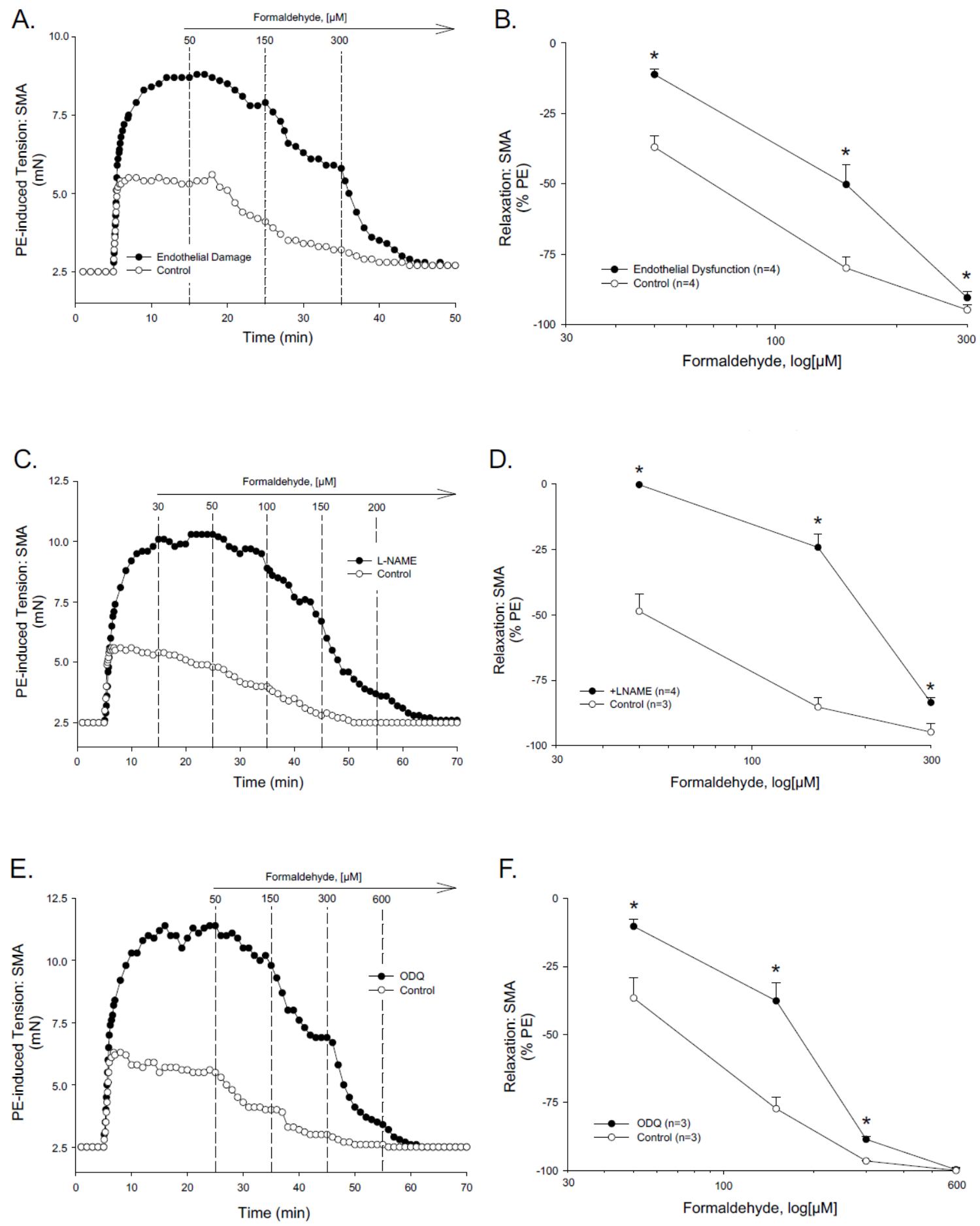
Fig. 12. Role of the TRPA1 channel in formaldehyde (FA)-induced relaxation in phenylephrine (PE) pre-contracted superior mesenteric artery (SMA). A) Representative traces of FA-stimulated relaxation of PE pre-contracted SMA in the absence (Control) and presence of the TRPA1 antagonist, A967079 $(3 \mu \mathrm{M})$, which was added after PE-induced contraction plateaued and prior to cumulative addition of FA in wild type (WT) SMA. B) Summary data of FA-induced relaxation in isolated SMA pre-contracted with PE in the absence and presence of TRPA1 antagonist. Immunofluorescence localization of TRPA1 in SMA (C) and in dorsal root ganglion (DRG; D). Formalin-fixed and paraffin-embedded sections of murine organs were stained with H\&E (Ci, Di); TRPA1 only (green; Cii) or DAPI only (blue, nuclear stain; Dii); TRPA1 antibody (green), isolectin (red, endothelium) and DAPI (Ciii) or TRPA1 antibody (red) and DAPI (Diii); and, TRPA1 antibody, TRPA1 blocking peptide, isolectin and DAPI (Civ) or TRPA1 antibody, TRPA1 blocking peptide, and DAPI (Div). L, lumen of SMA. All images were at 200x magnification (scale bar = $100 \mu \mathrm{m}$ ). Ei) Schematic depicting mouse mTrpa1 gene exons (E) 23 to 25 (with introns, I) and their length (in base pairs, bp). TRPA1 mRNA with the spliced exons 23 to 25 makes a 309 bp product as indicated by TRPA1 specific primers used. Eii) Agarose gel electrophoresis of PCR amplified product from 309 bp cDNA by qRT-PCR (101 bp product from SMA or DRG of WT mouse using TaqMan Assay for TRPA1; ct values indicated). Eiii) DNA sequence of 309 bp PCR product identified as TRPA1. Sequence of DRG PCR product (309 bp) was used as validation (data not shown). Values are means \pm SE of 3-4 preparations. ${ }^{*}, P<0.05$ vs Control. 
A.

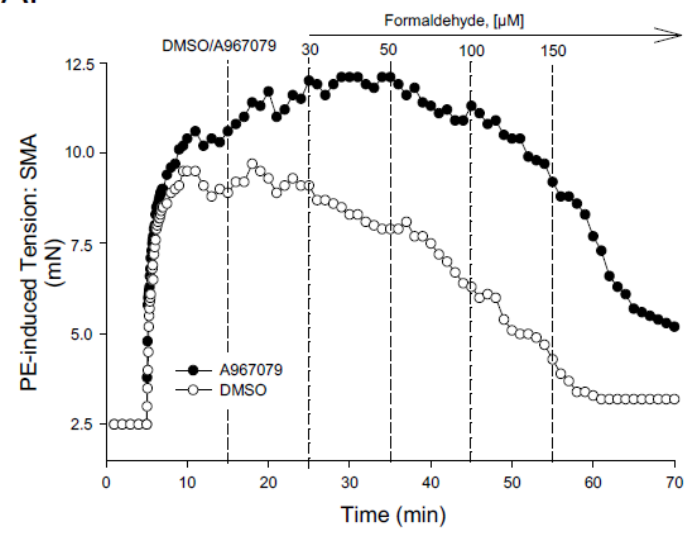

B.

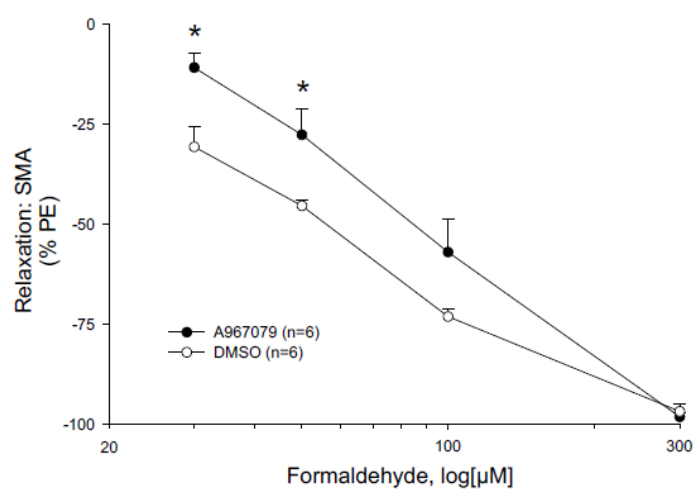

D.

C.
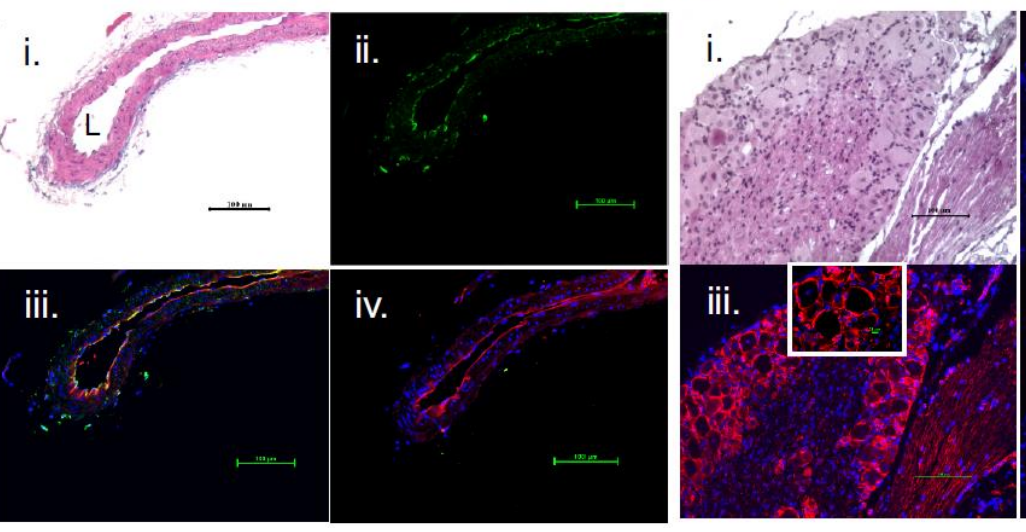

ii.

iv.
Ei.

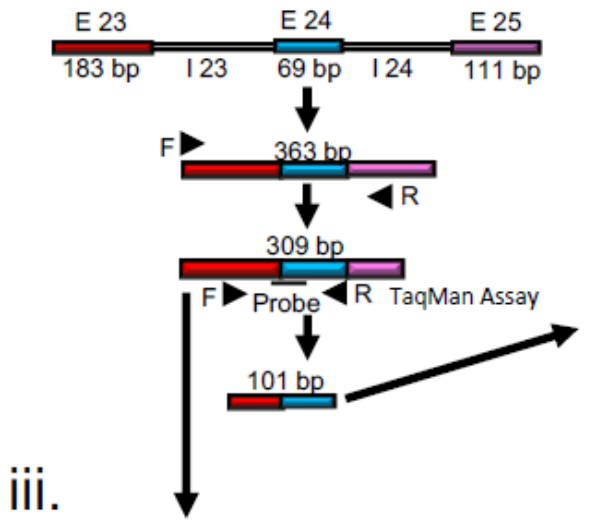

ii.

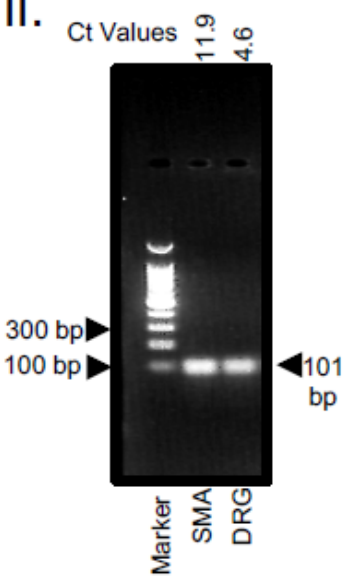

CGACAGCGAGTGCATGATGTGCGCAGCGTGATCTTGTCTCCAGATGCCTT CCGTCTCCCACTGTATCACCAGCCCACTCTGTACCCTGCCCTGACCTTTG GGCAGCTTATTGCCTTCACAATGTTTGTCCCAATTGTTCTCATGAACTTACT GATTGGCTTGGCGGTTGGGGACATTGCTGAGGTCCAGAAGCATGCGTCAT TGAAGAGGATTGCTATGCAGGTGGAACTTCATACCAACTTAGAAAAAAAGC TGCCACTCTGGTACTTACGCAAAGTGACAAAAAAAAAAAAAAAAAAAAAAAA AAACAAAACAAAAAAAAAAACAAAAAAAAAAAAAAAAAAAAAAAAAAACAAA Red: part of exon 23 
Fig. 13. Role of the endothelium and nitric oxide (NO) in the most sensitive component of cinnamaldehyde-induced $(\mathrm{CA})$ relaxation of phenylephrine (PE) precontracted superior mesenteric artery (SMA). A) Representative traces of concentration-dependent CA-stimulated relaxation of PE-induced contraction. B) Summary data of CA-induced relaxation in isolated SMA pre-contracted with PE in the presence and absence of functional endothelium. C) Summary data of CAinduced relaxation in isolated SMA pre-contracted with PE in the absence and presence of TRPA1 antagonist. D) Summary data of CA-induced relaxation in isolated SMA pre-contracted with PE in the absence and presence of L-NAME. E) Summary data of CA-stimulated relaxation of PE-induced contraction in the presence and absence of the guanylyl cyclase (GC) antagonist, ODQ $(3 \mu \mathrm{M})$, added to bath after PE-induced contraction plateaued and prior to cumulative addition of CA. Values are means \pm SE of 3-4 preparations. ${ }^{*}, P<0.05$ vs Control. 
A.

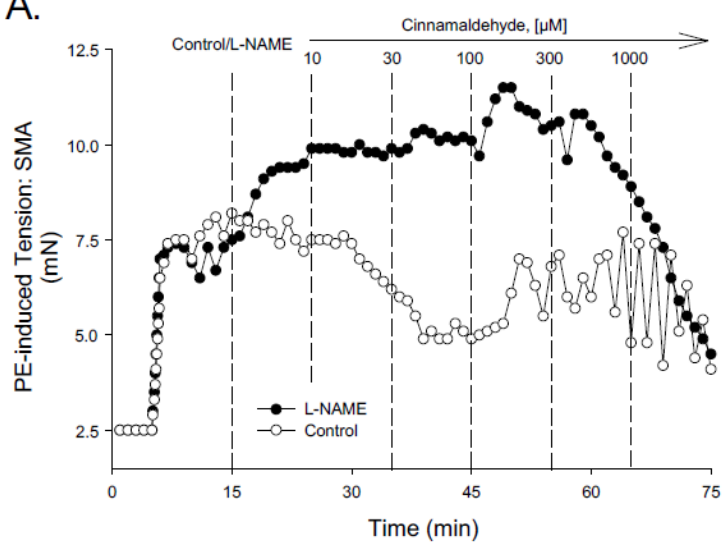

B.

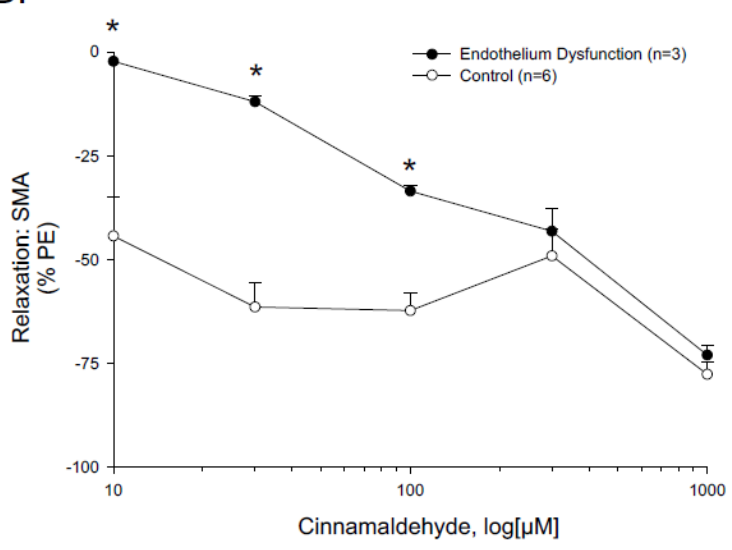

D.
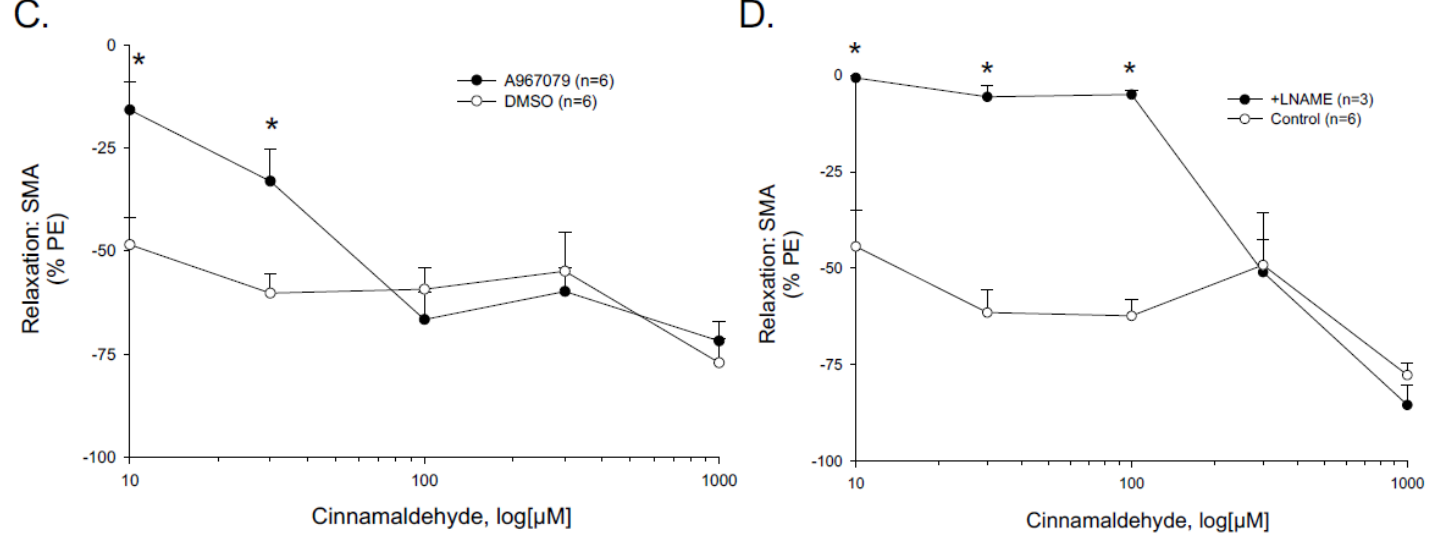

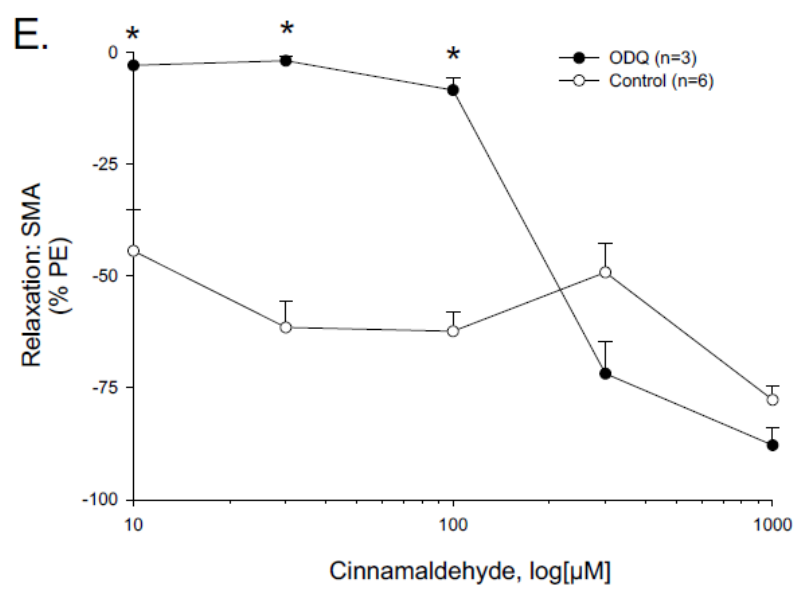


Fig. 14. Role of potassium channel $\left(\mathrm{K}_{\mathrm{IR}}\right)$ in formaldehyde $(\mathrm{FA})$-induced relaxation in phenylephrine (PE) pre-contracted blood vessels. A) Representative and B) summary curves of FA-induced relaxation in superior mesenteric artery (SMA) in the absence and presence of $\mathrm{BaCl}_{2}$. Summary curves of FA-induced relaxation in aorta in the absence and presence of $\mathbf{C}$ ) $\mathrm{BaCl}_{2}$ or $\mathbf{D}$ ) endothelium damage (ED). $\mathrm{BaCl}_{2}(1 \mathrm{mM})$ was added to PSS after stable PE-induced contraction and prior to cumulative addition of FA. ED was induced by 5 min of air perfusion. Values are means \pm SE of 3-4 preparations. *, $P<0.05$ vs Control. 

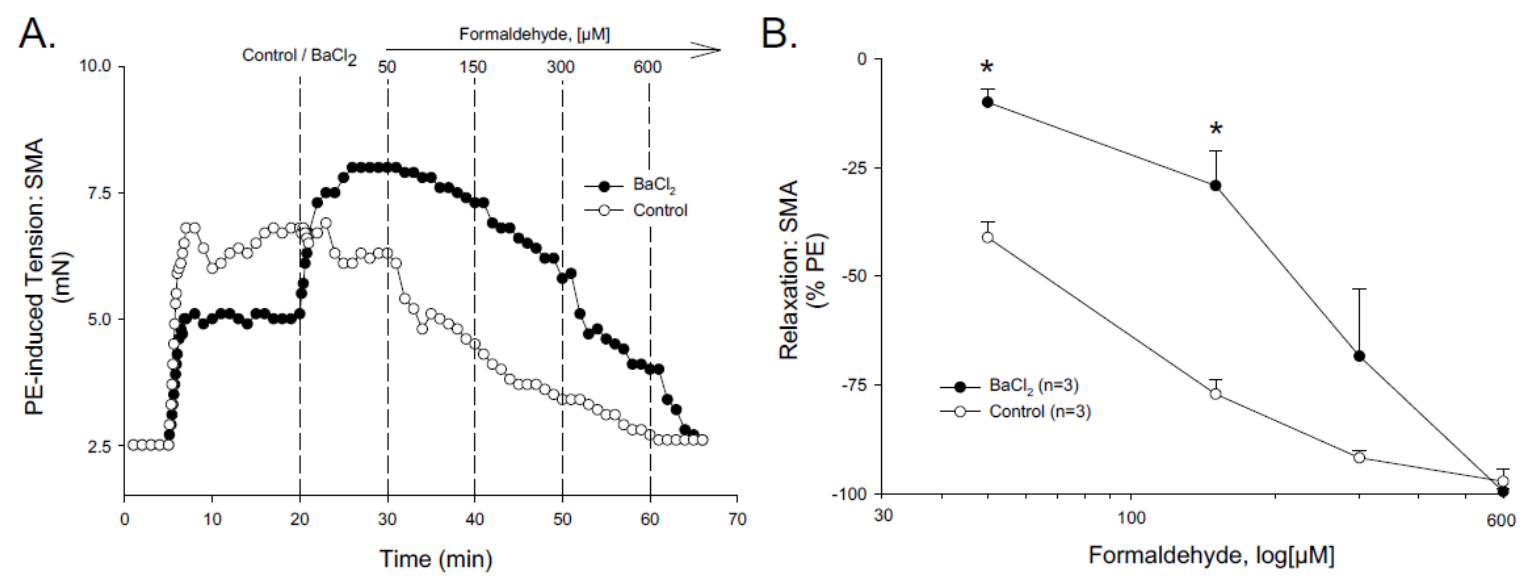

C.

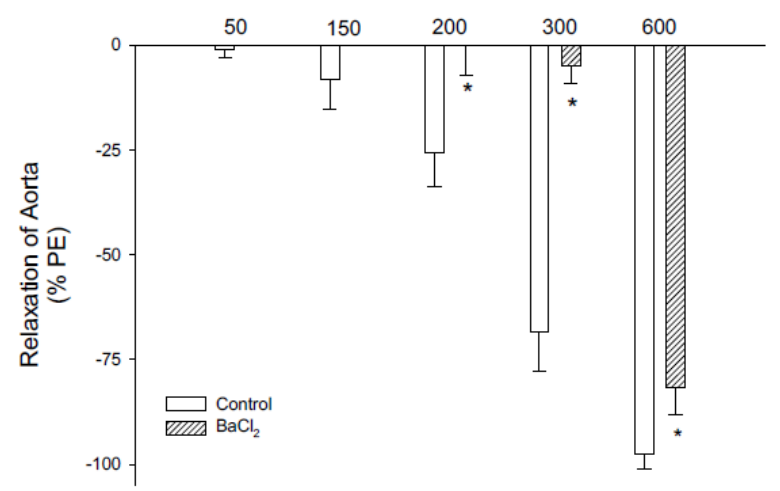

D.

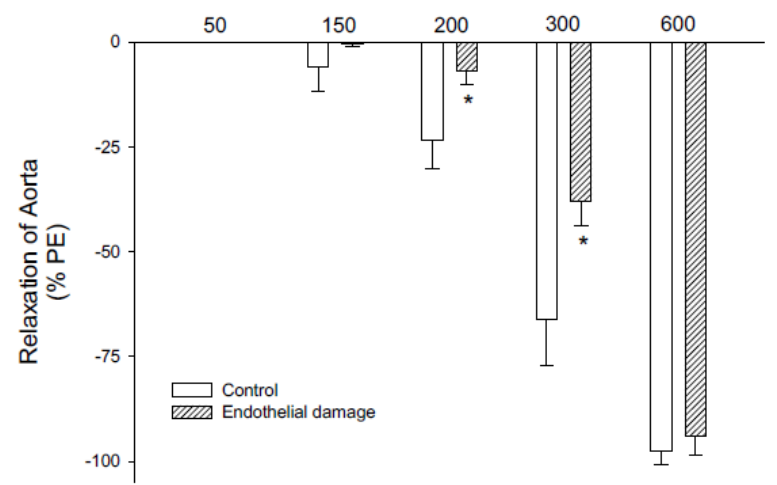


Table 8. The sensitivity of formaldehyde-induced vasorelaxation measured as the effective concentration inducing $50 \%$ relaxation $\left(\mathrm{EC}_{50}\right.$; in $\left.\mu \mathrm{M}\right)$ in isolated murine superior mesenteric artery (SMA; male) pre-contracted with PE in the absence and presence of functional endothelium and selective inhibitors of mediators of vasorelaxation.

\begin{tabular}{|c|c|c|}
\hline $\begin{array}{c}\text { SMA } \\
\text { (+treatment) }\end{array}$ & $\begin{array}{c}\mathrm{EC}_{50} \\
\text { (Control, PE+vehicle) }\end{array}$ & $\begin{array}{c}\mathrm{EC}_{50} \\
\text { (ED or PE+inhibitor) }\end{array}$ \\
\hline$+\mathrm{ED}$ & $61 \pm 8$ & $142 \pm 14^{*}$ \\
\hline$+\mathrm{LNAME}$ & $52 \pm 8$ & $207 \pm 10^{*}$ \\
\hline$+\mathrm{A} 967079$ & $52 \pm 6$ & $95 \pm 18^{*}$ \\
\hline$+\mathrm{ODQ}$ & $76 \pm 15$ & $182 \pm 16^{*}$ \\
\hline$+\mathrm{BaCl}_{2}$ & $63 \pm 10$ & $248 \pm 56^{*}$ \\
\hline$+\mathrm{TEA}$ & $75 \pm 3$ & $93 \pm 15$ \\
\hline
\end{tabular}

Values $=$ means \pm SE. Abbr.: PE, phenylephrine, $10 \mu \mathrm{M}$; ED, endothelial dysfunction induced by mechanical air perfusion; LNAME, $\mathrm{N}^{\omega}$-nitro-L-arginine methyl ester hydrochloride, $100 \mu \mathrm{M}$; A967079, TRPA1 antagonist, $3 \mu \mathrm{M}$; ODQ, 1h[1,2,4]oxadiazolo[4,3-a]quinoxalin-1-one, $\mathrm{BaCl}_{2}$, barium chloride, $1 \mathrm{mM}$; TEA, tetraethylammonium. $\mathrm{N}=3-6$ per group. ${ }^{*}, \mathrm{P}<0.05$ vs Control ( $\mathrm{PE}+$ vehicle). 


\section{Discussion}

To our knowledge, this is the first study to propose that FA may contribute to postprandial hyperemia via a TRPA1- an endothelium-mediated SMA vasodilation. Because rodents feed at night and sleep (fast) during the day, we measured and observed a circadian difference in urinary formate levels indicating endogenous FA level likely increases with feeding. As far as we know, this is a novel observation. Furthermore, we show that SMA is sensitive to FA significantly more so than aorta, and that FA not its metabolite, formate, stimulates a robust and reversible vasorelaxation. The SMA is a major arterial blood supply to the GI tract and it dilates postprandially in response to many stimuli e.g., nutrients, vagovagal reflex, NO, substance P, etc... (Chou \& Coatney, 1994; Lucchini, Saumet, Mei, \& Garnier, 1996). Although FA is oft considered a toxicant, its natural abundance in food, water and beverages (Committee on, 1981; D. J. Conklin, P. Haberzettl, et al., 2011) and as an abundant metabolic product make it a plausible stimulus of Gl blood flow. As this is the first suggestion of this physiological role, more research will be needed to establish FA as a bona fide contributor to postprandial hyperemia in vivo.

We show that there are 3 distinct pathways of FA-induced vasorelaxation in SMA (Fig. 15) and while this is fascinating in its own right, we focused on the mechanism of the most sensitive, and thus, most likely physiological pathway. FA-induced vasorelaxation requires a functional endothelium, TRPA1 channels, NOS (presumably eNOS), guanylyl cyclase (GC/cGMP) and KIR channels. Likely, the TRPA1 and NOS components are in endothelial cells, whereas the latter two 
components are likely present in vascular smooth muscle cells (VSMC). From these collective data, we propose a model featuring a stepwise sequence of events that is quite typical of agonists that elicit EDRF/NO such as ACh (see model in Fig. 15). That FA activates a TRPA1 channel likely present on the SMA endothelium is paralleled by FA action in rat aorta (Q. Zhang et al., 2018) and by systemically administered propofol - a general anesthetic that induces TRPA1-dependent hypotension (Sinha, Sinharoy, Bratz, \& Damron, 2015). Similarly, TRPA1 is a known target of formalin (solution of FA and methanol) that increases blood flow and pain (McNamara et al., 2007). With regard to formalin, we show that it is likely the FA in formalin and not the methanol, which can be metabolized to FA, that elicits vasorelaxation.

TRPA1 is a well-known target of unsaturated aldehydes, such as acrolein and crotonaldehyde (Andre et al., 2008; Bessac et al., 2008; D. J. Conklin, 2016) and cinnamaldehyde (Pozsgai et al., 2010), but its activation by FA is somewhat surprising given the proposed mechanism of unsaturated aldehyde-mediated activation via conjugation with TRPA1 $\mathrm{N}$-terminal free cysteines (Macpherson et al., 2007). In addition, several unsaturated aldehydes including acrolein, cinnamaldehyde and 4HNE induce sensitive and robust vasodilation in mesenteric arteries (Awe, Adeagbo, D'Souza, Bhatnagar, \& Conklin, 2006; Romero et al., 1997) and other blood vessels (Yanaga et al., 2006) and smooth muscle (Cheah et al., 2014) indicating perhaps a shared (universal) aldehyde-TRPA1 pathway.

In any case, TRPA1 is a cation (calcium) channel and a promiscuous receptor concentrated in sensory fibers (unmyelinated C-fibers) that mediate pain 
reception/transmission (nociceptors), and it is also are present in vasculature (Earley et al., 2009a; Sinharoy et al., 2017). Activation of peripheral sensory fiber TRPA1 leads to pain signaling and release of vasoactive peptides, substance P (SubP) and cGRP (Trevisan et al., 2016), which may contribute to increased vasodilation. We confirm localization of TRPA1 in mouse DRG and show specific staining in the endothelium of murine SMA but not in aorta. Functionally, the specific TRPA1 antagonist, A967079 $\left(\mathrm{IC}_{50}=289 \mathrm{nM}\right)$, significantly blocks the most sensitive component of the FA-induced relaxation and shifts the $\mathrm{EC}_{50}$ to the right quantitatively similar to that of endothelium disruption but less so than LNAME treatment -- further supporting a role of endothelial cell TRPA1. Above $150 \mu \mathrm{M} F A$ -- likely an unphysiological level of FA -- the TRPA1 antagonist did not affect FA relaxation reflecting a non-TRPA1 pathway(s), yet perhaps via TRPV1 or TRPV4 channels (Ma et al., 2008). We cannot rule out the possibility that additional components in the vascular wall (perivascular nerves, SubP, CGRP, etc...) also may contribute to overall FA-induced relaxation (Nilius, Appendino, \& Owsianik, 2012).

FA-induced vasorelaxation is well-documented for over 4 decades, yet the complex mechanisms of this phenomenon are only now becoming clearer. In contrast to the idea that FA induces endothelial dysfunction (Yu \& Deng, 1998), our data indicate that endothelial dysfunction itself likely would block only the physiological (sensitive) component of FA-induced vasorelaxation. We did not observe evidence of FA-induced endothelial dysfunction even after exposing SMA to $1,000 \mu \mathrm{M} F A$. Moreover, in isolated human CABG blood vessels with endothelial 
dysfunction, FA still induces relaxation but in a far less sensitive manner $\left(\mathrm{EC}_{50}\right.$, $315 \pm 0 \mu \mathrm{M})(\mathrm{D}$. J. Conklin et al., 2004) than that present in healthy mice $(\approx 65 \mu \mathrm{M}$; this study).

It is clear that the vascular effects of FA ex vivo are not a result of FA metabolism into formate (primary metabolite). For example, formate addition (up to $2.1 \mathrm{mM}$ ) fails to evoke a strong vascular response, and thus, oxidative metabolism of FA regulates FA levels and subsequently any FA-evoked physiological vascular responses. Similarly, formate fails to recapitulate formalininduced vasodepressor response in rats in vivo (Strubelt et al., 1990). Because FA metabolism involves a reduced glutathione (GSH)- and FALDH-dependent (aka ADH3) hemi-thioacetal formation (Kaiser, Holmquist, Vallee, \& Jornvall, 1991), this process may be more affected by changes in oxidant load and GSH depletion in the vascular wall or in neurons, for example (Materazzi et al., 2012). As shown previously, deficiency in glutathione-dependent metabolism of acrolein (i.e., GSTP-null mice) increases susceptibility to acrolein-induced endothelial dysfunction in vivo and in vitro (D. J. Conklin, Haberzettl, et al., 2009a). Whether or not FALDH deficiency or GSH depletion likewise alters vascular sensitivity to FA-induced relaxation (or toxicity) remains to be tested.

In conclusion, we describe a sensitive mechanism of FA-induced vasorelaxation that is sequentially dependent on an endothelium-localized TRPA1 receptor (EDRF/NO) and a VSMC GC/PKG/KIR pathway in SMA. We hypothesize that this sensitive pathway is only a part of the postprandial hyperemia reflex that increases blood flow to the GI tract to augment digestion and nutrient absorption. 
Fig. 15. A cartoon depicting the mechanisms by which formaldehyde (FA) induces vasorelaxation in SMA precontracted by phenylephrine (PE), thromboxane $A_{2}$ analog (U46,619) and $60 \mathrm{mM}$ potassium solution (60K). FA appeared to induce a sensitive and sequential mechanism that begins with opening of Transient Receptor Potential Ankyrin 1 (TRPA1) cation channels, $\mathrm{Ca}^{2+}$ entry into endothelial cell, eNOS activation, NO formation, GC activation and cGMP formation with Protein Kinase $G$ (PKG) mediated opening of $K_{I R}$ channels leading to VSMC hyperpolarization and relaxation. Several of these steps were significantly impaired by a selective antagonist or treatment, including TRPA1 (A967079), eNOS (LNAME), endothelium (impaired by air perfusion), guanylyl cyclase (GC; ODQ) and $\mathrm{K}_{\mathrm{IR}}$ channel $\left(\mathrm{BaCl}_{2}\right)$. As demonstrated, this sequential pathway was sensitive to exogenous FA $(30-150 \mu \mathrm{M})$ in the physiological range that was altered at least $2-3 x$ during feeding. Other less sensitive yet equally efficacious pathways of vasorelaxation included VSMC hyperpolarization and a voltage-insensitive mechanism that reversed $60 \mathrm{~K}$-induced contraction implicating closure of VSMC $\mathrm{Ca}^{2+}$ channels. 


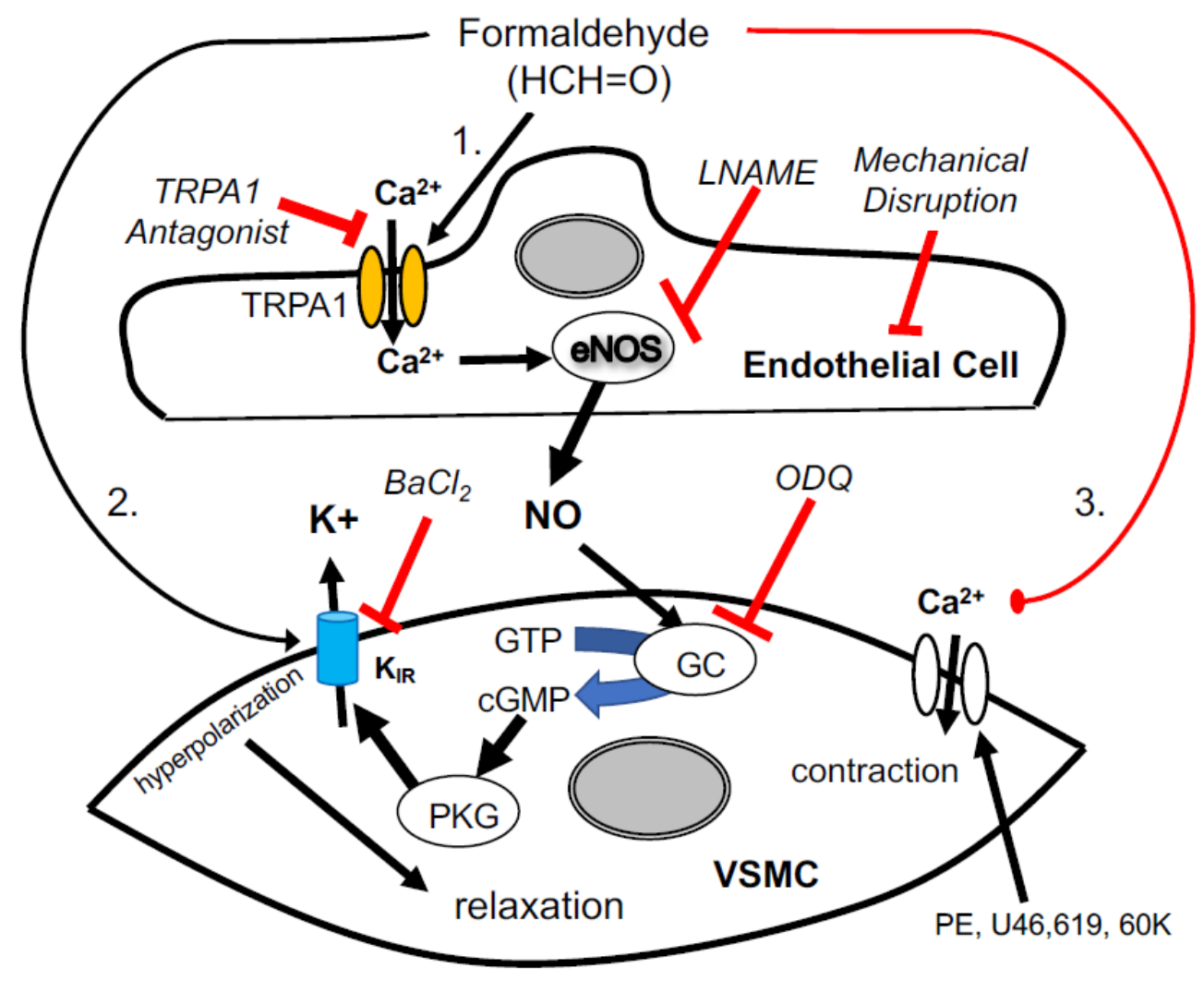




\title{
CHAPTER IV
}

\section{ACETALDEHYDE INDUCES AN ENDOTHELIUM-DEPENDENT RELAXATION OF SUPERIOR MESENTERIC ARTERY: \\ POTENTIAL ROLE IN POSTPRANDIAL HYPERMIA}

\begin{abstract}
Acetaldehyde (AA) is a small, ubiquitous compound present in foods, beverages, as a gas phase combustion product, and also endogenouslygenerated from metabolism as from ethanol $(\mathrm{EtOH})$. Acetate is a short chain fatty acid derived from AA oxidation, and acetate levels were significantly higher in urine collected overnight with food provided ad libitum compared with urine collected after 9h fasting. Because feeding increases gastrointestinal blood flow, we explored the direct effects of AA (and acetate) in isolated murine superior mesenteric artery (SMA). Over the concentration range of 1-100 mM, AA strongly and reversibly relaxed agonist-induced contractions of SMA including phenylephrine (PE), thromboxane $A_{2}$ analog $(U 46,619)$ and high potassium (High $\mathrm{K}^{+}$) without toxicity. The sensitivity (EC50) but not the efficacy (>90\% relaxation of PE-precontraction) of AA-induced relaxations was dependent on blood vessel (SMA was $3 x$ more sensitive than aorta) and contractile agonist (PE EC50=3.3 \pm 0.4 $\mathrm{mM} ; \mathrm{U} 46,619 \mathrm{EC} 50=14.9 \pm 1.5 \mathrm{mM}$; High $\mathrm{K}+\mathrm{EC} 50=17.7 \pm 0.5 \mathrm{mM}$ ) yet
\end{abstract}


independent of circadian cycle and sex. The most sensitive component of the AAinduced relaxation was inhibited significantly by: 1) mechanically-impaired endothelium; 2) nitric oxide synthase (NOS) inhibitor (L-NAME); and, 3) a guanylyl cyclase (GC) inhibitor (ODQ). Both acetate and EtOH stimulated much weaker relaxations in SMA than did AA, yet these relaxations were significantly inhibited by L-NAME as well. Neither EtOH nor acetate relaxed pre-contracted aorta. Although neither cyanamide, a non-specific aldehyde dehydrogenase (ALDH) enzyme inhibitor, nor Alda-1, a specific activator of ALDH2 activity, had any effect on either sensitivity or efficacy of AA-induced relaxation in SMA, cyanamide significantly blocked both $\mathrm{EtOH}$ - and acetate-induced relaxations in SMA implicating a role of ALDH activity in vasorelaxation. These data show that AA relaxes SMA via an endothelium- and NO-dependent mechanism supporting the idea that AA may contribute to the physiological reflex of post-prandial hyperemia via vasodilatation of mesenteric vasculature.

\section{Introduction}

Acetaldehyde (AA) is the second smallest aldehyde, and the concentration of $A A$ in the blood is typically low due to high levels of aldehyde dehydrogenase (ALDH) activity throughout the body. AA is present in food and beverages and is from ethanol $(\mathrm{EtOH})$ metabolism, which can be a major exogenous source of systemic AA and AA may also enter via inhalation of vehicle exhaust-derived aerosols (D. J. Conklin, P. Haberzettl, et al., 2011; Ogunwale et al., 2017). Typically, AA levels range in the blood from 1-100 $\mu \mathrm{M}$, and can reach $>1 \mathrm{mM}$ with 
excessive alcohol ingestion especially in individuals with an aldehyde dehydrogenase 2, ALDH2 ${ }^{\star} 2$ mutation, e.g., common in SE Asians (Tsukamoto et al., 1991; Vasiliou \& Nebert, 2005). Blood levels of AA are constantly changing due to metabolism and exposures (eg., feeding and inhilation) (Hobara et al., 1985) and thus, what physiological role AA plays in vascular control has remained uncertain.

A variety of direct effects of exogenous AA on cardiovascular targets have been described, yet a distinct role of $A A$ in cardiovascular physiology is missing. For example, intra-arterial or intravenous AA reduces heart rate (bradycardia), decreases arterial pressure (hypotension) and causes arrhythmias in animals, but these are considered toxicological effects due to exceedingly high doses administered directly into the blood (Egle, Hudgins, \& Lai, 1973; M. A. Green \& Egle, 1983). Nonetheless, AA is ubiquitous, and, it is thought that AA generated from EtOH contributes to alcohol-induced cardiomyopathy (Ren \& Brown, 2000; Tan et al., 2012). Recently, it was shown that perfusion of a low level of AA (50 $\mu \mathrm{M})$ into an isolated rat heart induces a cardioprotective effect against ischemiareperfusion indicating that endogenous AA levels likely are bioactive (Ueta et al., 2018). It is well-known that $A A$ induces concentration-dependent relaxation in many different isolated blood vessels (Altura \& Altura, 1982, 1987), yet the relevance of this relaxation to vascular physiology is unclear. Yet, AA is considered relevant to the well-known phenomenon of $\mathrm{EtOH}$-induced 'flushing' (increased blood flow and redness in facial skin flushing) in people with an 
ALDH2*2 mutation that reduces ALDH2 activity, and thus, limits the rate of metabolism of AA to acetate (Scarino, Tardif, \& Charbonneau, 2009).

Despite myriad sources and well-known cardiovascular effects of AA, few studies address the physiological role of AA in vascular control. To address this gap in knowledge, we measured urine levels of acetate, the primary metabolite of AA, to assess fluctuations in endogenous AA levels in mice. Urine levels of acetate increased $\approx 2-3$ times overnight after feeding compared with urine levels during a daytime fast, and thus, it appeared that feeding increased endogenous AA levels (directly or indirectly). The gastrointestinal vasculature, thus, represents a likely target of AA levels after feeding when gut blood flow reflexively increases (Chou \& Coatney, 1994; Takagi et al., 1988). To test this idea, isolated superior mesenteric artery (SMA) and aorta were exposed to AA across a physiological to supraphysiological range (1-100 mM). The most sensitive AA-induced relaxation was in the SMA (1-30 mM), and it was dependent on: a functional endothelium, NO formation, and guanylyl cyclase activation. Because $\mathrm{EtOH}$ is metabolized to AA by alcohol dehydrogenases, and AA is metabolized by ALDH to acetate, the vascular effects of EtOH and acetate in the absence and presence of an ALDH inhibitor (cyanamide) and ALDH2 activator (Alda1) were also assessed. EtOH and acetate (up to $30 \mathrm{mM}$ ) elicited less robust relaxations (less than $50 \%$ of AA-induced relaxation), yet these relaxations were NO- and ALDH-dependent in SMA indicating intrinsic overlap with AA's mechanism of action. For many reasons, tobacco and $\mathrm{EtOH}$ are often consumed together, so effect of nicotine on AAinduced relaxation also was tested (Abreu-Villaca, Manhaes, Krahe, Filgueiras, \& 
Ribeiro-Carvalho, 2017). Collectively, these findings support the idea that increased levels of AA due to feeding (and associated drinking of EtOH) likely contribute to reflex-driven increased gastrointestinal blood flow after feeding (aka 'postprandial hyperemia') through a sensitive, robust, reversible, endothelium- and NO-dependent process.

\section{Material and Methods}

Chemicals and Solutions.

Reagent grade chemicals were purchased from Sigma-Aldrich or other commercial sources as indicated: A967079 (AdooQ); acetaldehyde; acetate; acetylcholine chloride (ACh); Alda1 and cyanamide (gift of Dr. B.G. Hill, Univ. Louisville); 1h-[1,2,4]oxadiazolo[4,3-a]quinoxalin-1-one (ODQ); ethanol (100\%); $\mathrm{N}^{\omega}{ }^{\omega}$-nitro-L-arginine methyl ester hydrochloride (L-NAME); L-phenylephrine hydrochloride (PE); sodium nitroprusside (SNP); U46,619 (thromboxane $A_{2}$ analog); and, 2,3,4,5-pentafluorobenzyle bromide (PFBBr).

Krebs physiological salt solution (PSS) for SMA was (in $\mathrm{mM}$ ): $\mathrm{NaCl}, 119$; $\mathrm{KCl}, 4.7 ; \mathrm{MgCl}_{2}, 1.2 ; \mathrm{KH}_{2} \mathrm{PO}_{4}, 1.2 ; \mathrm{NaHCO}_{3}$, 24; glucose, 7.0; $\mathrm{pH}$ 7.4. Krebs PSS for aorta was (in $\mathrm{mM}$ ): $\mathrm{NaCl} 118, \mathrm{KCl} 4.7, \mathrm{CaCl}_{2} 2.5, \mathrm{KH}_{2} \mathrm{PO}_{4}$ 1.2, $\mathrm{MgSO}_{4}$ 1.2, $\mathrm{NaHCO}_{3}$ 12.5, and glucose 5.5; $\mathrm{pH}$ 7.4. High $\mathrm{K}^{+} \mathrm{PSS}$ (High K; $60 \mathrm{mM}$ ) was prepared by substituting equimolar $\mathrm{K}^{+}$for $\mathrm{Na}^{+}$.

Animals. 
Wild type C57BL/6J mice from in house breeding pairs or purchased (The Jackson Laboratory, Bar Harbor, ME) (12-20 weeks old; 25-35g) were used in these studies. Aldose reductase (AR) null mice were from a breeding colony at Univ. of Louisville (Wetzelberger et al., 2010). Mice were treated according to American Physiological Society Guiding Principles in the Care and Use of Animals, and all protocols were approved by University of Louisville Institutional Animal Care and Use Committee. Mice were housed under pathogen-free conditions in the Univ. of Louisville vivarium under controlled temperature and $12 \mathrm{~h}$ light:12h dark cycle. Mice were provided a standard chow diet (Rodent Diet 5010, 4.5\% fat by weight, LabDiet; St. Louis, MO).

\section{Urine Collection and Acetate Measurement.}

Urine samples were collected, centrifuged (2,500 RPM, $\left.10 \mathrm{~min}, 4^{\circ} \mathrm{C}\right)$, decanted, and stored at $-80^{\circ} \mathrm{C}$ until analysis (D. J. Conklin et al., 2017). Each mouse was held $(<5 \mathrm{sec})$ and the mouth was gently touched with the sipper tube of a water bottle filled with D-glucose/saccharin solution (w/v; 3.0\%/0.125\%; Sigma) immediately prior to a $6 \mathrm{~h}$ fast. After $6 \mathrm{~h}$ fast, each mouse was placed in a metabolic cage (Harvard Apparatus) with ab libitum access to glucose/saccharin solution in water without access to food for $3 \mathrm{~h}$ to collect urine (in $4^{\circ} \mathrm{C}$ water-jacketed organ baths). In total, mice were fasted during daytime for 9-h. After fasted urine collection, mice then were provided food and glucose/saccharin solution ab libitum for an overnight urine collection $(\mathrm{O} / \mathrm{N})$. For urine samples, volumes were recorded, samples centrifuged to sediment food/feces, decanted, and stored at $-80^{\circ} \mathrm{C}$ until analysis (D. J. Conklin et al., 2017). 
The primary urinary metabolite of AA is acetate, and it was measured using GC-MS as described previously (Kage et al., 2004; Lamarre et al., 2014) with minor modifications (Conklin et al., 2018). Briefly, urine $(50 \mu \mathrm{L})$ was mixed with sodium phosphate $\left(20 \mu \mathrm{L} 0.5 \mathrm{M}, \mathrm{pH}\right.$ 8.0) containing internal standard $\left({ }^{13} \mathrm{C}\right.$-acetate, $2.3 \mathrm{mM})$. Samples were incubated with $\operatorname{PFBBr}(130 \mu \mathrm{L}, 0.1 \mathrm{M})$ for 15 min at $60^{\circ} \mathrm{C}$, extracted with hexane $(330 \mu \mathrm{L})$, and analyzed by GC-MS in electron ionization (EI) mode. Six-point calibration curve was used to calculate the concentration of acetate. Results were corrected for the natural abundance of ${ }^{13} \mathrm{C}$ isotope. Acetate level was normalized to urinary creatinine $(\mathrm{mg})$ to account for urine dilution (Lorkiewicz et al., 2019).

Isolated Superior Mesenteric Artery (SMA) and Aorta.

Mice were anesthetized with sodium pentobarbital $(0.1 \mathrm{ml}, 150 \mathrm{mg} / \mathrm{kg}$, i.p.), and SMA and aorta were removed via mid-ventral thoracotomy. Thoracic aorta rings (3-4 mm) were hung on stainless steel hooks in 15-ml water-jacketed organ baths and SMA rings $(2 \mathrm{~mm})$ were hung on tungsten wire $(75 \mu \mathrm{m}$ dia. $)$ in $5-\mathrm{ml}$ heated organ baths (MultiWire Myograph System 620M, DMT, Denmark) in PSS bubbled with $95 \% \mathrm{O}_{2}: 5 \% \mathrm{CO}_{2}$ at $37{ }^{\circ} \mathrm{C}$. After 10 min without tension, aorta rings were equilibrated to $\approx 1 \mathrm{~g}$ loading tension over $30 \mathrm{~min}$ and SMA rings were equilibrated to $\approx 0.25 \mathrm{~g}$ loading tension over $1 \mathrm{~h}$. All rings were stimulated with $60 \mathrm{~K}$ to test for viability, washed 3 times with PSS over $30 \mathrm{~min}$, and re-equilibrated to appropriate resting tension (Jin, Jagatheesan, et al., 2019).

AA-Induced Relaxation in Isolated Superior Mesenteric Artery (SMA) and Aorta: 
Acetaldehyde (1-100 mM) was added to organ baths containing either aorta or SMAs pre-contracted with 1 of 3 contractile agonists: phenylephrine (PE, 10 $\mu \mathrm{M}), \mathrm{U} 46,619(0.1 \mu \mathrm{M})$, or High $\mathrm{K}^{+}(60 \mathrm{mM})$. The efficacy of AA-induced relaxation was calculated as the maximal \% reduction in agonist-induced contraction ( $\left.E_{\max }\right)$. The sensitivity of agonist-induced relaxation was calculated as the effective concentration producing $50 \%$ response $\left(\mathrm{EC}_{50}\right)$, i.e., cumulative concentration responses were normalized to $100 \%$ with interpolation of EC $_{50}$ (D. J. Conklin, Haberzettl, et al., 2009b). To assess whether time of day affected SMA response to AA, mice were euthanized both in day and in night cycle (Jin, Jagatheesan, et al., 2019).

Role of AA Metabolism and Effects of EtOH and Acetate (AA metabolite):

Because $\mathrm{EtOH}$ is a known source of $\mathrm{AA}$, we tested whether $\mathrm{EtOH}$ alone (up to $1 \%$, equivalent to amount present in $100 \mathrm{mM} \mathrm{AA}$ ) mimicked $\mathrm{AA}$-induced relaxation. Because AA is oxidized to acetate (primary urinary metabolite), we tested whether acetate (1-100 mM) alone could induce vasorelaxation in PE precontracted aorta and SMA. To test the specific role of ALDH to AA-induced vasorelaxation, we used a non-specific ALDH enzyme inhibitor (cyanamide, $1 \mathrm{mM}$ ) and an activator of ALDH2 (Alda-1, $25 \mu \mathrm{M}$ ) (Chen et al., 2008; Srivastava et al., 2001). In addition, to test for role of acetaldehyde reductive metabolism, SMA and aorta were isolated from aldose reductase (AR) null mice.

Role of Endothelium, Nitric Oxide Synthase (NOS), and cGMP: 
To evaluate the role of NOS, L-NAME $(0.1 \mathrm{mM})$ was added prior (15 $\mathrm{min})$ to addition of PE $(10 \mu \mathrm{M}), \mathrm{U} 46,619(0.1 \mu \mathrm{M})$ or High $\mathrm{K}^{+}(60 \mathrm{mM})$. Pre-contracted SMA rings were then relaxed with cumulative concentrations of $A A$. The role of the endothelium was assessed as vasorelaxation to increasing AA concentrations in aorta and in SMA with intact and injured endothelium. The endothelium was mechanically-injured by air perfusion, and effective impairment was confirmed by near complete abolition (>95\%) of ACh-induced dilation of PE pre-contracted blood vessels (Jin, Jagatheesan, et al., 2019). To assess whether cGMP was involved in AA-induced vasorelaxation, isolated blood vessels were pre-incubated with ODQ $(3 \mu \mathrm{M})$ to inhibit guanylyl cyclase $(\mathrm{GC})$ and subsequent formation of cGMP (Jiang et al., 2015).

Role of Transient Receptor Potential Ankyrin 1 (TRPA1):

Because formaldehyde induces a sensitive TRPA1-dependent relaxation in isolated SMA, we tested whether the TRPA1 antagonist (A967079, $3 \mu \mathrm{M})$ would alter AA-induced relaxation in PE-precontracted SMA (Jin, Jagatheesan, et al., 2019).

Role of nicotine:

Because most cigarette and e-cigarette contains nicotine, we tested whether nicotine would affect AA-induced relaxation in PE-precontracted SMA.

Statistical Analyses.

Data are expressed as means \pm SE. When comparing two groups, a $t$-test was used (paired or unpaired as appropriate). Multiple groups were compared 
using Kruskal-Wallis ANOVA followed by Bonferroni post-hoc tests. Statistical significance was assumed when $\mathrm{P}<0.05$.

\section{Results}

Acetate: the primary metabolite of acetaldehyde $(A A)$ is increased with feeding.

Acetate is the primary oxidation metabolite of AA, and thus, urinary acetate is a product of endogenous AA metabolism. We measured urinary acetate using GC-MS, and we found that feeding (overnight) significantly increased urinary levels of acetate indicating that a major source of endogenous AA likely is either directly from food or related to feeding, e.g., metabolism of food constituents, intermediary metabolism, etc... In female and male C57BL/6J mice, a daytime fast $(9 \mathrm{~h})$ resulted in a basal level of urinary acetate that overnight feeding was increased by 2-3 times (Table 9). Thus, we infer that feeding overnight increased AA levels that resulted in increased acetate excretion in urine.

\section{AA-induced relaxation.}

Because feeding increases gastrointestinal (Gl) blood flow, we investigated whether AA would relax superior mesenteric artery (SMA) - a major conduit for increased Gl blood flow with feeding (Chou \& Coatney, 1994; Lucchini et al., 1996). In fact, $\mathrm{AA}$ (1 to $100 \mathrm{mM}$ ) induced a concentration-dependent, robust, repeatable and agonist-dependent relaxation in isolated SMA and aorta of female and male mice (Fig. 16; Table 10). Regardless of agonist (PE, U46,619, High $\mathrm{K}^{+}$), AA relaxed precontraction tension by $>90 \%$ in SMA (Fig. 16A, B) and in aorta (Table 
10; PE only), yet when added to an uncontracted blood vessel, AA did not affect basal tone (aka myogenic tone; data not shown). Blood vessels sensitivity of male SMA was circadian-independent, and sensitivity of female SMA in daytime was less sensitive to male SMA sensitivity (either day or night cycle) (Fig. 16 C, D; Table 10), and thus, subsequent experiments to probe the mechanisms of AAinduced relaxation were conducted using only SMA isolated from male mice in the daytime (lights on). Collectively, AA-induced relaxation was repeatable (even up to $100 \mathrm{mM}$ ) without any loss of efficacy or any induced change in endotheliumdependent ACh-induced relaxation indicating little overt vasotoxicity of AA even at supra-physiological levels (data not shown).

Role of endothelium, nitric oxide synthase (NOS), and GC in AA-induced vasorelaxation.

The sensitivity of AA-induced relaxation (but not efficacy) was agonist-dependent (PE<U46,619=High K+; Fig. 16 A, B; Table 10) and indicated at least 2 distinct mechanisms were operative. We showed the most sensitive component of the AAinduced relaxation was significantly rightward-shifted by mechanical disruption of endothelium in SMA (air perfusion impaired $>90 \%$ of ACh-induced relaxation) (Fig. 17 A, B; Tables 11, 12). This effect was similar to the effect of L-NAME incubation, which further implicated endothelium-derived NO (Fig. 17 C, D; Tables 11, 12). Because NO stimulates guanylyl cyclase (GC) to generate cGMP in smooth muscle cells, we used ODQ, an irreversible inhibitor of GC, and it also rightwardshifted the AA-induced relaxation (Fig. 17 E, F; Tables 11, 12). Collectively, these data show that the most sensitive component of the AA-induced relaxation was 
dependent on a functional endothelium, NOS (likely eNOS) and an NO-mediated activation of GC, but at high concentrations, the relaxation of AA was not affected (Table 11). Thus, L-NAME shifted the sensitivity of AA-induced relaxation in SMA pre-contracted with PE ( $3.3 \pm 0.3$ vs $6.3 \pm 0.6 \mathrm{mM})$, but did not alter efficacy (Emax). Similarly, it neither affected the sensitivity nor efficacy of AA-induced relaxation in U46,619- (17.2 \pm 0.3 vs $14.9 \pm 1.5 \mathrm{mM})$ or High K- $(18.4 \pm 0.4$ vs $17.7 \pm 0.5 \mathrm{mM})$ precontracted SMA (Fig. 18A, B; Table 10). These data support a role of endothelium-derived NO in the most sensitive component of AA-induced relaxation in PE-precontracted SMA.

Ethanol-, acetaldehyde- and acetate-induced relaxations.

Because $A A$ is formed from EtOH by alcohol dehydrogenase $(A D H)$, and AA is metabolized by ALDH to acetate, it is possible that the vascular effects of $\mathrm{EtOH}$ or acetate are due to AA. To address this possibility, EtOH and acetate were cumulatively added (up to $100 \mathrm{mM}$ ) in PE pre-contracted SMA and aorta. Ethanol is known to induce vasodilation in several blood vessels, yet it is unclear if the effect is due to formation of AA. Ethanol relaxed PE-precontracted SMA weakly (Emax about $40 \%$ of PE precontraction) compared with AA (Fig. 19A, B). Ethanol did not relax PE pre-contracted aorta even at $100 \mathrm{mM}$, in fact, it increased tension primarily at $100 \mathrm{mM}$ (Fig. 20A). Acetate also induced weak relaxation and a slight contraction at $100 \mathrm{mM}$ in PE-precontracted SMA (Fig. 19A, B, C). L-NAME treatment significantly reduced both $\mathrm{EtOH}$ - and acetate-induced relaxations of SMA (Fig. 19D, E; Table 11). Although both EtOH- and acetate-induced 
significantly weaker relaxations in SMA than did AA, the NO dependence of these relaxations was a shared mechanism of action with $A A$.

Role of AA metabolism: Effects of Cyanamide and Alda1 and AR.

To investigate the possible role of metabolism in AA-induced responses, cyanamide and Alda-1, ALDH inhibitor and ALDH2 activator, respectively, and an aldose reductase (AR) null mouse model were used. Surprisingly, and despite vasoactivity of their own, cyanamide (increased tension, 10-20\%) and Alda-1 (decreased tension, 10-20\%), neither compound had any significant effect on AAinduced relaxation in PE-precontracted SMA (Fig. 21A, B; Table 13). However, the sensitivity of the AA-induced relaxation of SMA was rightward shifted significantly in AR-null mice $\left(\mathrm{EC}_{50}, 6.9 \pm 1.0 \mathrm{mM}, \mathrm{n}=3\right)$ although no effect on maximal relaxation was observed $\left(E_{\max }, 78.1 \pm 4.0 \%\right)$. Despite lack of a strong effect of ALDH modulators on AA's vasoactivity, cyanamide significantly blocked the relaxation of $\mathrm{EtOH}$, and Alda1 slightly enhanced the $\mathrm{EtOH}$ relaxation (Fig. 21C, D; Table 13). Similarly, cyanamide significantly blocked the relaxation of acetate, although Alda1 was without effect on the relaxation of acetate (Fig. 21E, F; Table 13). These data support a potential regulatory role of ALDH activity in $\mathrm{EtOH}$ - and acetate-induced relaxations in SMA (see Fig. 22).

Role of Transient Receptor Potential Ankyrin 1 (TRPA1):

Although formaldehyde induces a sensitive TRPA1- and endotheliumdependent relaxation in isolated SMA, AA-induced relaxation in PE-precontracted 
SMA was unaffected by addition of the TRPA1 antagonist (A967079, $1 \mu \mathrm{M}$ ) (Tables $\mathbf{1 1}, \mathbf{1 2}$ ) indicating this pathway is not activated in a similar way by AA.

Nicotine and $A A$ :

In tobacco users, EtOH, and thus, AA, may be present in blood simultaneously with nicotine, so we also tested whether nicotine altered AAinduced relaxation in SMA. Nicotine alone $(1 \mu \mathrm{M})$ had no effect in PE-contracted SMA, and also had no effect on subsequent AA-induced vasorelaxation (Tables 11, 12). 
Table 9. Levels of urinary acetate following a daytime fast and overnight feeding in (A) female and (B) male C57BL/6J mice.

A. Females

\begin{tabular}{|c|c|c|}
\hline & \multicolumn{2}{|c|}{ Condition } \\
\hline Urinary Metabolite & Daytime Fast & Overnight Feeding \\
\hline Acetate $(\mu \mathrm{g} / \mathrm{ml})$ & $2.76 \pm 0.27$ & $24.52 \pm 4.89^{\star}$ \\
\hline Creatinine $(\mathrm{mg} / \mathrm{dl})$ & $3.19 \pm 0.41$ & $8.74 \pm 1.52$ \\
\hline $\begin{array}{c}\text { Acetate }(\mu \mathrm{g} / \mathrm{mg} \\
\text { creatinine })\end{array}$ & $91.11 \pm 6.61$ & $296.82 \pm 32.95^{\star}$ \\
\hline
\end{tabular}

B. Males

\begin{tabular}{|c|c|c|}
\hline & \multicolumn{2}{|c|}{ Condition } \\
\hline Urinary Metabolite & Daytime Fast & Overnight Feeding \\
\hline Acetate $(\mu \mathrm{g} / \mathrm{ml})$ & $4.71 \pm 0.66$ & $8.54 \pm 2.34^{*}$ \\
\hline Creatinine $(\mathrm{mg} / \mathrm{dl})$ & $6.52 \pm 1.16$ & $5.67 \pm 1.15$ \\
\hline $\begin{array}{c}\text { Acetate }(\mu \mathrm{g} / \mathrm{mg} \\
\text { creatinine })\end{array}$ & $79.56 \pm 7.49$ & $158.57 \pm 32.16^{\star}$ \\
\hline
\end{tabular}

Values $=$ means $\pm S E . N=11-12$ per group. ${ }^{*}, \mathrm{P}<0.05$ vs fasting. 
Table 10. The effective concentration inducing $50 \%$ relaxation $\left(E_{50}\right.$; in $\left.\mathrm{mM}\right)$ of acetaldehyde-induced vasorelaxation in isolated murine superior mesenteric artery (SMA; female and male) and thoracic aorta (male only) with different contractile agonists.

\begin{tabular}{|c|c|c|c|}
\hline \multirow{2}{*}{$\begin{array}{c}\text { Blood Vessel } \\
\text { (sex, time of } \\
\text { day) }\end{array}$} & \multicolumn{3}{|c|}{ EC $_{50}$ by precontraction agonist } \\
\cline { 2 - 4 } & PE & U46,619 & High K \\
\hline SMA (f, day) & $6.0 \pm 0.6^{*}$ & -- & -- \\
\hline SMA (m, day) & $3.3 \pm 0.4$ & $14.9 \pm 1.5^{\star}$ & $17.7 \pm 0.5^{\star}$ \\
\hline $\begin{array}{c}\text { SMA (m, day) } \\
\text { +LNAME }\end{array}$ & $6.2 \pm 0.2^{*}$ & $17.2 \pm 0.2$ & $18.4 \pm 0.4$ \\
\hline SMA (m, night) & $4.3 \pm 0.8$ & -- & -- \\
\hline Aorta (m) & $15.9 \pm 3.0^{*}$ & & \\
\hline
\end{tabular}

Values = means \pm SE. Abbr.: PE, phenylephrine, [10 $\mu \mathrm{M}]$; U46,619, thromboxane $\mathrm{A}_{2}$ analog, [0.1 $\mu \mathrm{M}$ ]; High $\mathrm{K}$, high potassium solution, [60 mM K+]; --, did not perform experiment. $\mathrm{N}=3-10$ per group. ${ }^{*}, \mathrm{P}<0.05$ vs $\mathrm{EC}_{50}$ of "SMA (male, day)" PE agonist (i.e., baseline control). 
Fig. 16. Acetaldehyde (AA) induced relaxation in superior mesenteric artery (SMA). A) Representative traces of concentration-dependent relaxation induced by cumulative addition of AA (1-100 mM) in isolated SMA pre-contracted by one of 3 agonists: phenylephrine (PE); thromboxane $A_{2}$ analog (U46,619); or, high potassium $\left(\mathrm{High} \mathrm{K}^{+}\right)$. B) Summary data of the efficacy ( $\mathrm{E}_{\max } \%$ relaxation) of AAinduced relaxation in isolated $\mathrm{PE}, \mathrm{U} 46,619$ and $\mathrm{High} \mathrm{K}^{+}$pre-contracted SMA. C) Summary data of the efficacy $\left(E_{\max }\right)$ and of the (D) sensitivity $\left(E_{50}\right)$ of AA-induced relaxation in isolated PE-precontracted SMA from male mice euthanized either during day or night time. Values are means \pm SE of SMA from 3-4 mice. 
A.

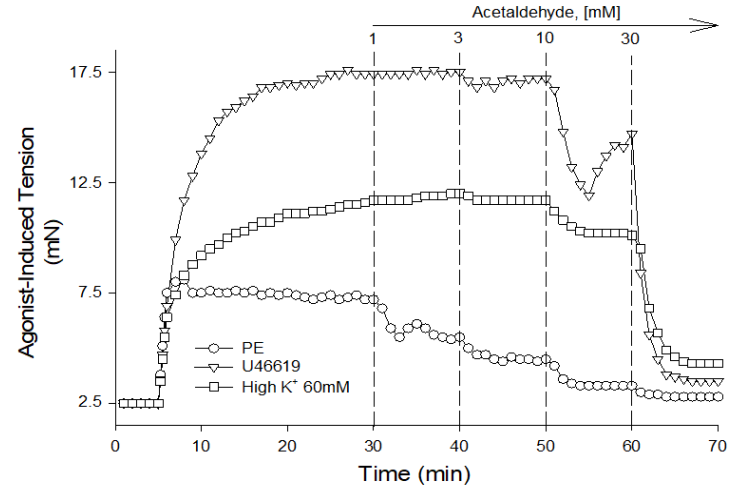

C.

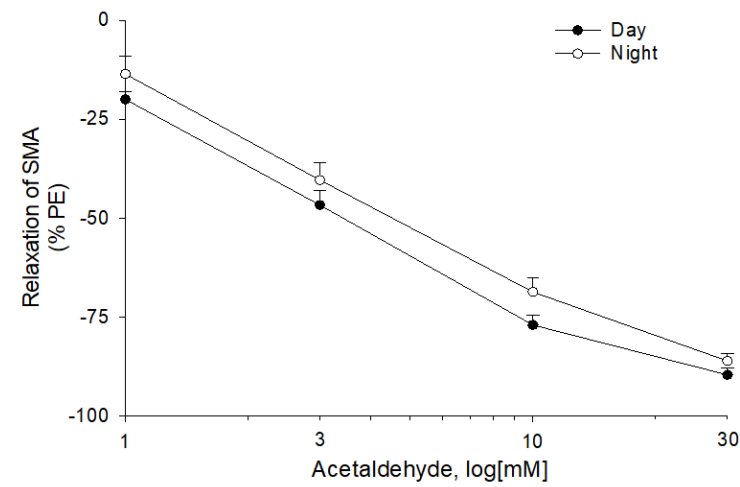

B.

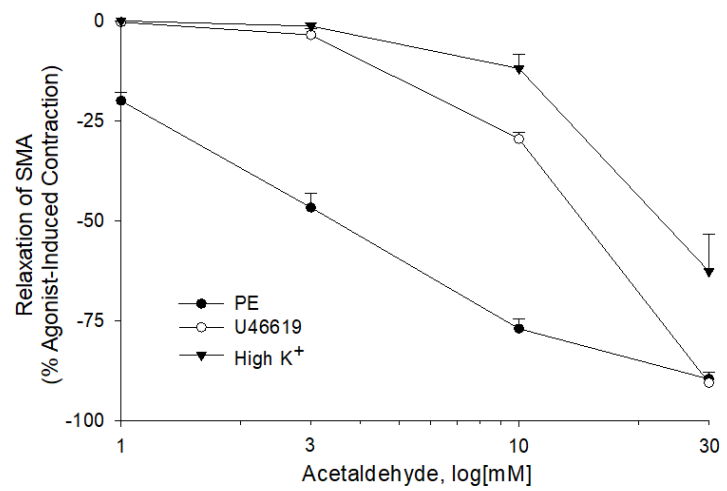

D.

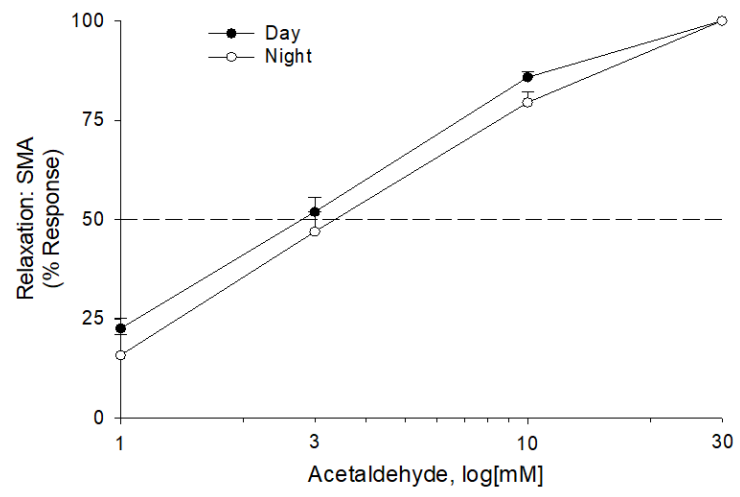


Fig. 17. Role of the endothelium and nitric oxide (NO) in acetaldehyde-induced (AA) relaxation of SMA. A, B) Representative traces and summary data of AAstimulated relaxation of PE-induced contraction in SMA with intact endothelium or with endothelium impairment due to 15 min of air perfusion. C, D) Representative traces and summary data of AA-stimulated relaxation of PE-induced contraction in the absence of and presence of NOS inhibitor, L-NAME. E, F) Representative traces and summary data of AA-induced relaxation of PE-induced contraction in the absence of and presence of the guanylyl cyclase antagonist, ODQ ( $3 \mu \mathrm{M})$, added to bath after PE-induced contraction stabilized and prior to cumulative addition of $A A$. Values are means \pm SE of SMA from 3-8 mice as indicated. *, $P<0.05$ vs Control. 
A.

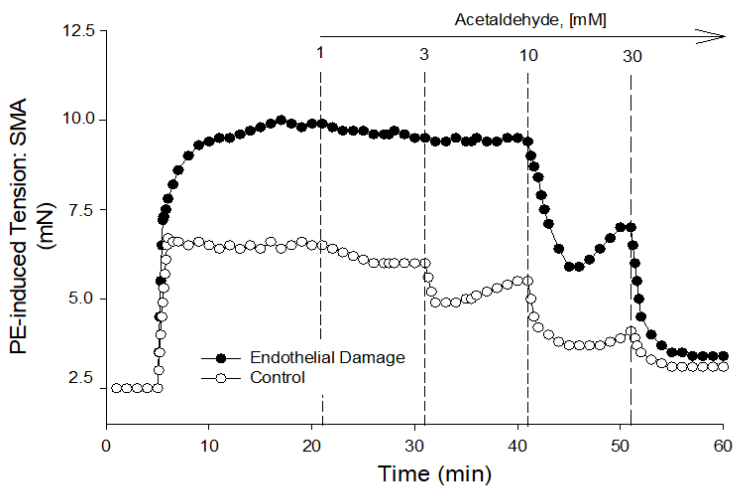

C.

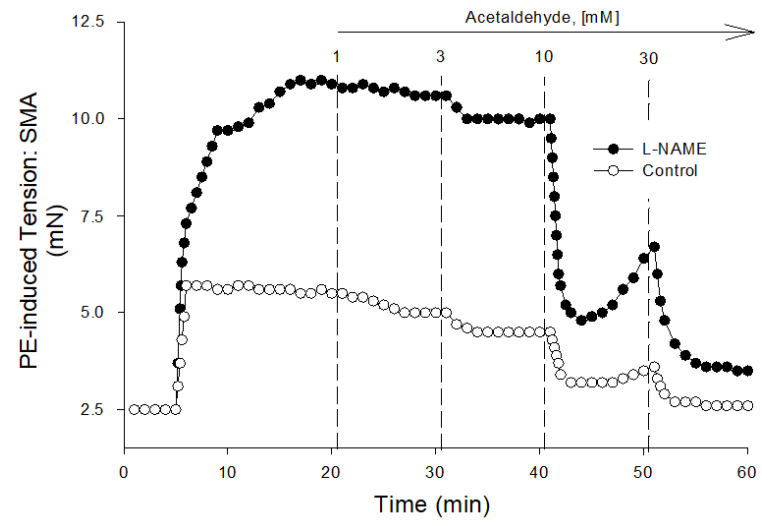

E.

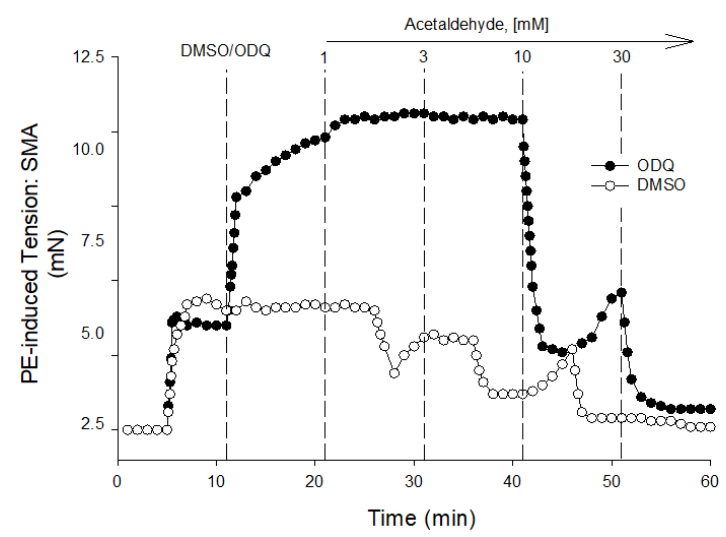

B.

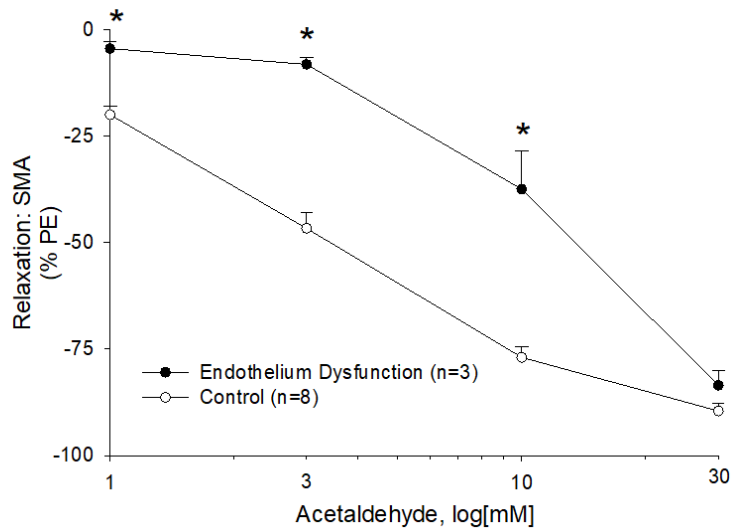

D.

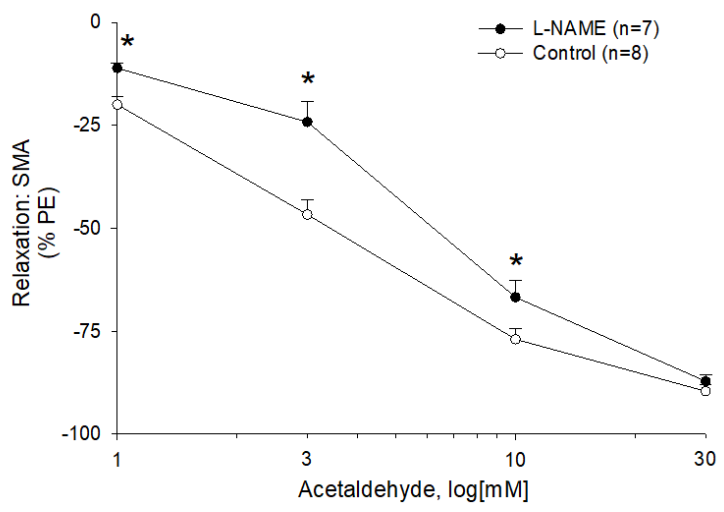

F.

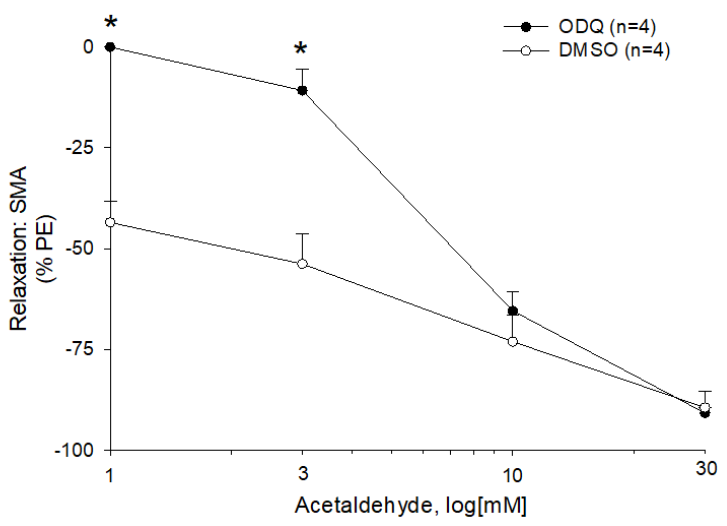


Table 11. Efficacy (maximal relaxation, $E_{\max }$ ) of acetaldehyde- $(\mathrm{AA}), \mathrm{EtOH}$ - and acetate-induced relaxations of PE-precontracted superior mesenteric artery (SMA) and aorta without and with inhibitor treatments.

\begin{tabular}{|c|c|c|c|c|c|}
\hline \multirow{2}{*}{ Compound } & \multicolumn{2}{|c|}{$[\mathrm{mM}]$} & \multirow{2}{*}{ Treatment } & \multicolumn{2}{c|}{$E_{\max }$ (\% relaxation) } \\
\cline { 2 - 3 } \cline { 5 - 6 } & SMA & Aorta & & SMA & Aorta \\
\hline AA & $1-30$ & $1-100$ & Control & $89.6 \pm 1.7$ & $91.6 \pm 6.7$ \\
\hline AA & $1-30$ & -- & ED & $83.5 \pm 3.4$ & -- \\
\hline AA & $1-30$ & -- & +LNAME & $87.1 \pm 4.0$ & -- \\
\hline AA & $1-30$ & -- & +ODQ & $90.8 \pm 1.8$ & -- \\
\hline AA & $1-30$ & -- & + A967079 & $90.6 \pm 4.0$ & -- \\
\hline EtOH & $1-30$ & $1-100$ & Control & $39.4 \pm 3.1$ & 0 \\
\hline EtOH & $1-30$ & -- & +LNAME & $8.1 \pm 1.2^{*}$ & -- \\
\hline Acetate & $1-30$ & $1-100$ & Control & $42.9 \pm 5.9$ & 0 \\
\hline Acetate & $1-30$ & -- & +LNAME & $16.0 \pm 8.2^{*}$ & -- \\
\hline
\end{tabular}

Values $=$ means \pm SE. Abbr.: PE, phenylephrine, $10 \mu \mathrm{M}$; ED, endothelial dysfunction induced by mechanical air perfusion; LNAME, $\mathrm{N}^{\omega}$-nitro-L-arginine methyl ester hydrochloride, $100 \mu \mathrm{M}$; A967079, TRPA1 antagonist, $3 \mu \mathrm{M}$; ODQ, 1h[1,2,4]oxadiazolo[4,3-a]quinoxalin-1-one. $\mathrm{N}=3-6$ per group. * $\mathrm{P}<0.05$ vs untreated and matched Control. 
Table 12. The effective concentration inducing $50 \%$ relaxation $\left(E C_{50}\right.$; in $\left.\mathrm{mM}\right)$ of acetaldehyde- (AA), EtOH- or acetate-induced vasorelaxation in isolated murine superior mesenteric artery (SMA; male mice only) pre-contracted with PE in the absence and presence of functional endothelium or selective inhibitors/compounds.

\begin{tabular}{|c|c|c|c|}
\hline $\begin{array}{l}\text { Compound } \\
{[1-30 \mathrm{mM}]}\end{array}$ & $\begin{array}{c}\text { Control EC } \\
\text { (vehicle) }\end{array}$ & Treatment & Treatment $\mathrm{EC}_{50}$ \\
\hline$A A$ & $3.9 \pm 0.6$ & $+\mathrm{ED}$ & $12.4 \pm 2.1^{*}$ \\
\hline AA & $2.9 \pm 0.4$ & +LNAME & $6.2 \pm 0.6^{*}$ \\
\hline AA & $2.1 \pm 0.9$ & $+\mathrm{ODQ}$ & $7.4 \pm 0.9^{*}$ \\
\hline AA & $2.9 \pm 0.6$ & $+\mathrm{A} 967079$ & $2.8 \pm 0.6$ \\
\hline $\mathrm{EtOH}$ & $3.2 \pm 1.5$ & +LNAME & $\mathrm{X}$ \\
\hline acetate & $6.9 \pm 3.0$ & +LNAME & $x$ \\
\hline
\end{tabular}

Values $=$ means \pm SE. Abbr.: PE, phenylephrine, $10 \mu \mathrm{M}$; ED, endothelium dysfunction induced by mechanical air perfusion; LNAME, $\mathrm{N}^{\omega}$-nitro-L-arginine methyl ester hydrochloride, $100 \mu \mathrm{M}$; ODQ, 1h-[1,2,4]oxadiazolo[4,3-a]quinoxalin1-one; A967079, TRPA1 antagonist, $3 \mu \mathrm{M}$; X, could not calculate due to low \% relaxation (see Table 3). $\quad \mathrm{N}=3-6$ mice used per group. ${ }^{*}, \mathrm{P}<0.05$ vs Control (vehicle). 
Fig. 18. Role of the nitric oxide (NO) in acetaldehyde-induced relaxations of SMA. AA (1-100 mM) induced relaxation of isolated SMA pre-contracted by one of 3 agonists: phenylephrine (PE); thromboxane $A_{2}$ analog $(U 46,619)$; or, high potassium $\left(\right.$ High $\mathrm{K}^{+}$). Summary data of the $(\mathbf{A})$ efficacy (\% relaxation) and of the (B) sensitivity of AA-induced relaxation in isolated PE-precontracted SMA in the absence and presence of L-NAME. Values are means \pm SE of SMA from 3-8 mice. 
A.

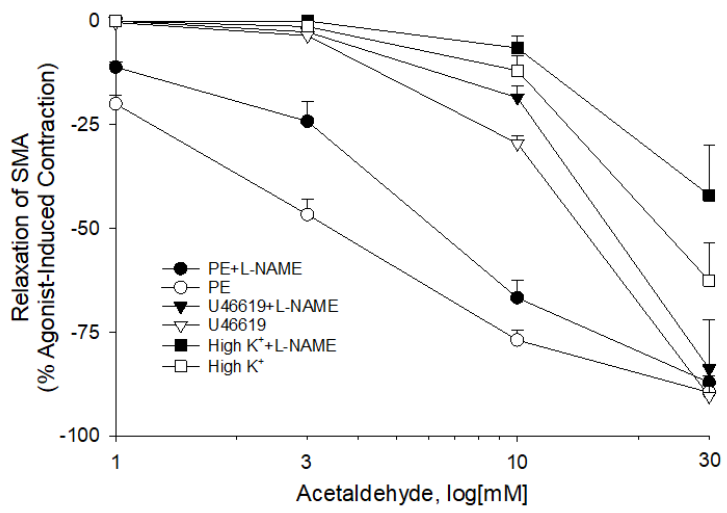

B.

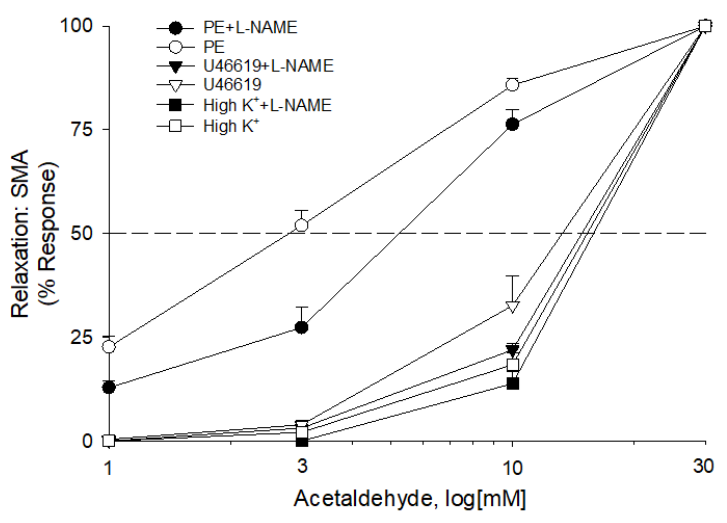


Fig. 19. The vascular effects of ethanol (EtOH), acetaldehyde (AA) and acetate in SMA. Role of the nitric oxide (NO) in ethanol- and acetate-induced relaxations of SMA. A) Representative traces of EtOH-, AA- and acetate-induced relaxations in PE pre-contracted SMA. Summary data of EtOH-, AA- and acetate-induced relaxations in PE pre-contracted SMA plotted as measures of efficacy (B, \% PE contraction) and sensitivity (C, converted to $100 \%$ response). Concentration dependent relaxations induced by either (D) ethanol or (E) acetate of phenylephrine (PE) pre-contracted superior mesenteric artery (SMA) in the absence and presence of L-NAME. Values are means \pm SE of SMA from 3 mice as indicated. ${ }^{*}, \mathrm{P}<0.05$ vs Control. 
A.

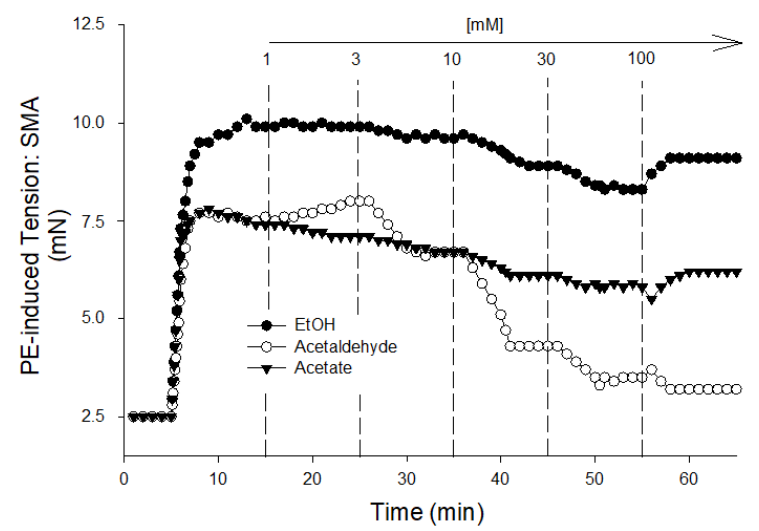

C.

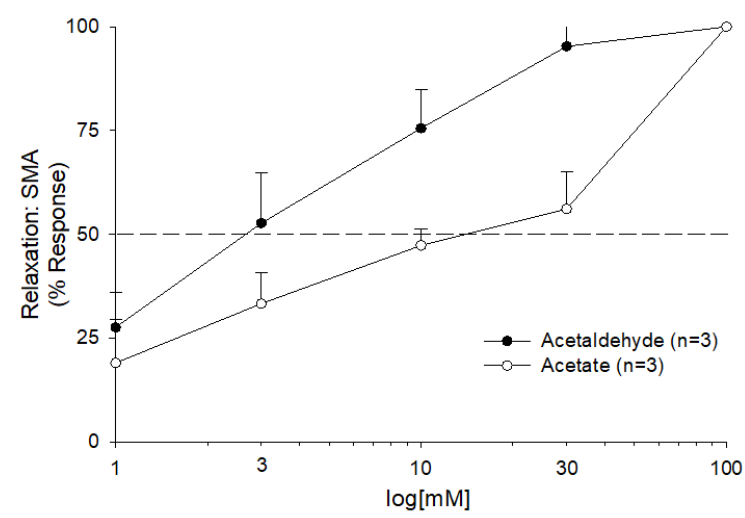

E.

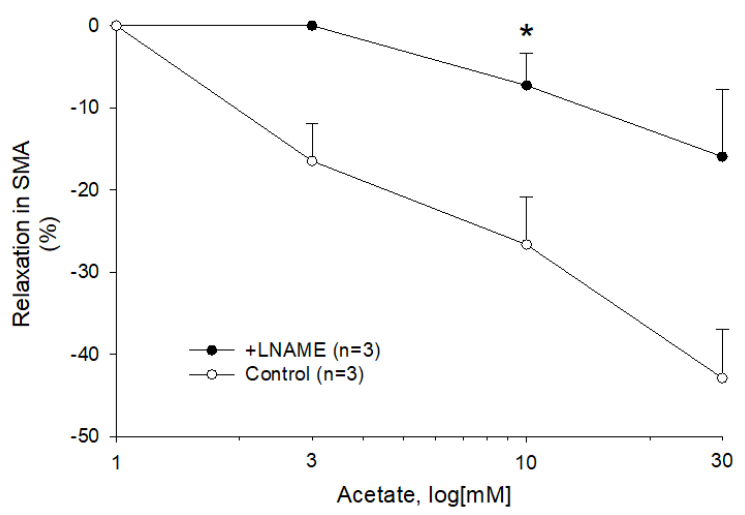

B.

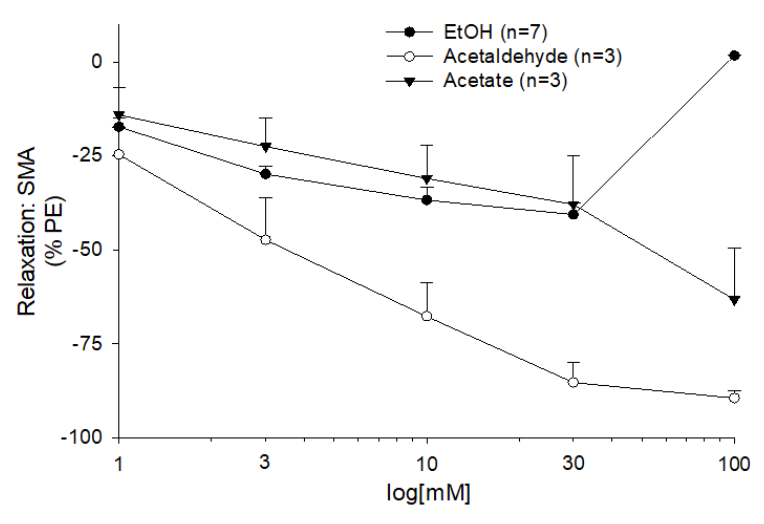

D.

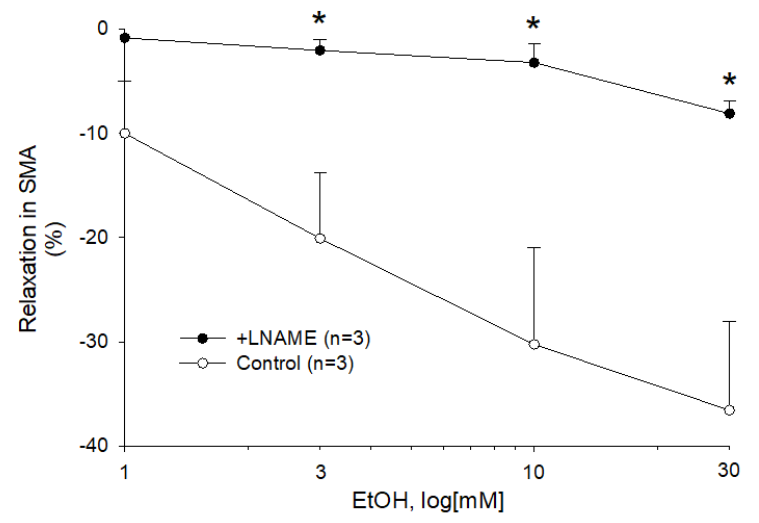


Fig. 20. The vascular effects of ethanol $(\mathrm{EtOH})$, acetaldehyde (AA) and acetate in aorta. A) Representative traces of EtOH-, AA- and acetate-induced responses in PE pre-contracted aorta. Summary data of AA-induced relaxations in PE precontracted aorta plotted as measures of efficacy (B, \% PE contraction) and sensitivity (C, converted to $100 \%$ response). Values are means \pm SE of aorta from 5 mice as indicated. 
A.

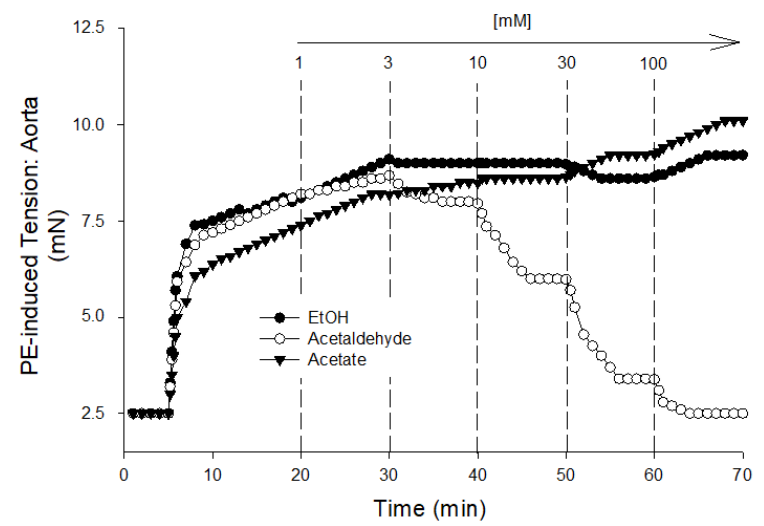

C.

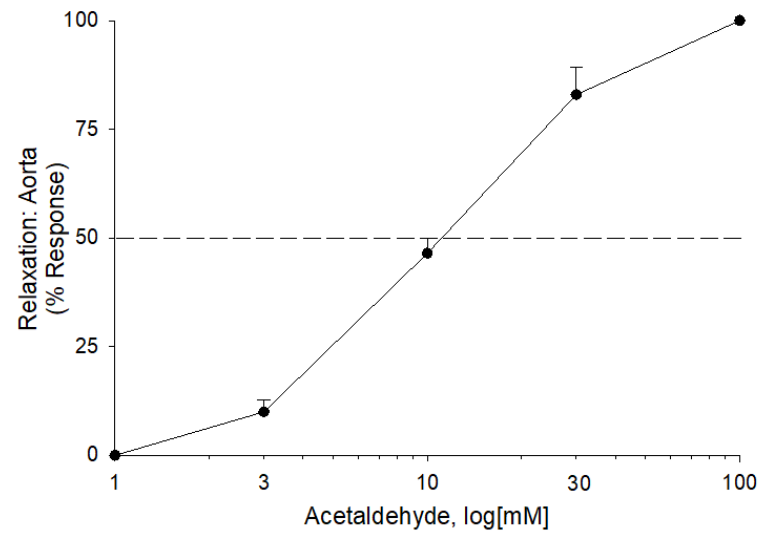

B.

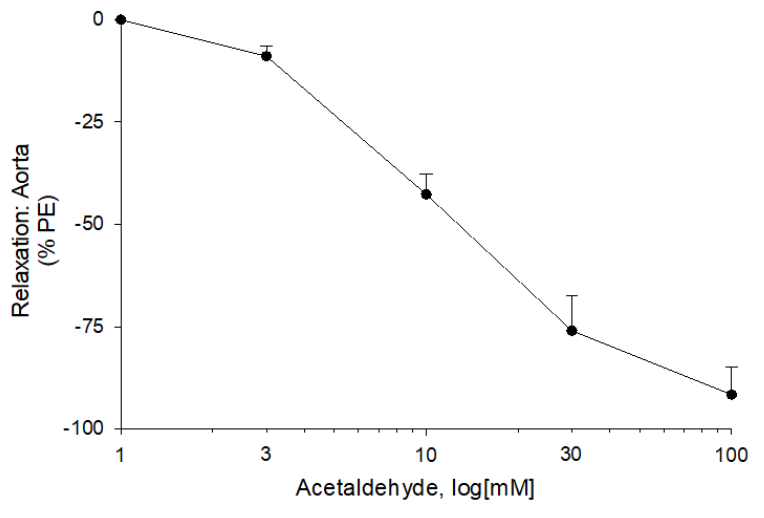


Fig. 21. Role of aldehyde dehydrogenase (ALDH) metabolism in vascular responses to $\mathrm{EtOH}, \mathrm{AA}$ and acetate. Representative traces and summary data, respectively, of vasorelaxant effects of $\mathrm{AA}(\mathbf{A}, \mathbf{B}), \mathrm{EtOH}(\mathbf{C}, \mathbf{D})$ and acetate $(\mathbf{E}, \mathbf{F})$ in PE pre-contracted SMA of WT mice in the absence and presence of either cyanamide (ALDH enzyme inhibitor, $1 \mathrm{mM}$ ) or Alda1 (ALDH2 activator, $25 \mu \mathrm{M}$ ). The vasorelaxant effects of AA in AR-null mice (A). Values are means \pm SE of SMA from 3-8 mice as indicated. ${ }^{*}, P<0.05$ vs Control. 
A.

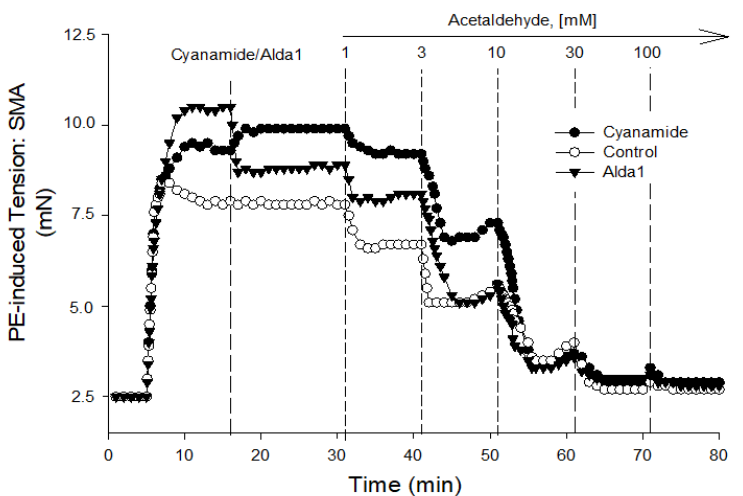

C.

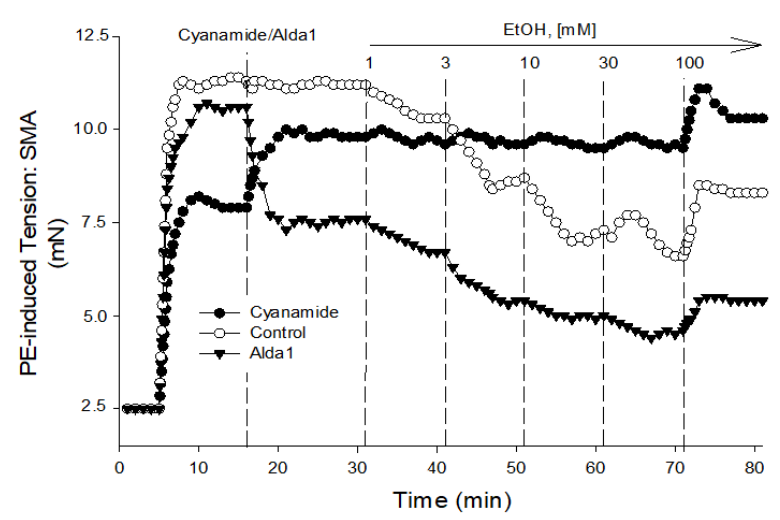

E.

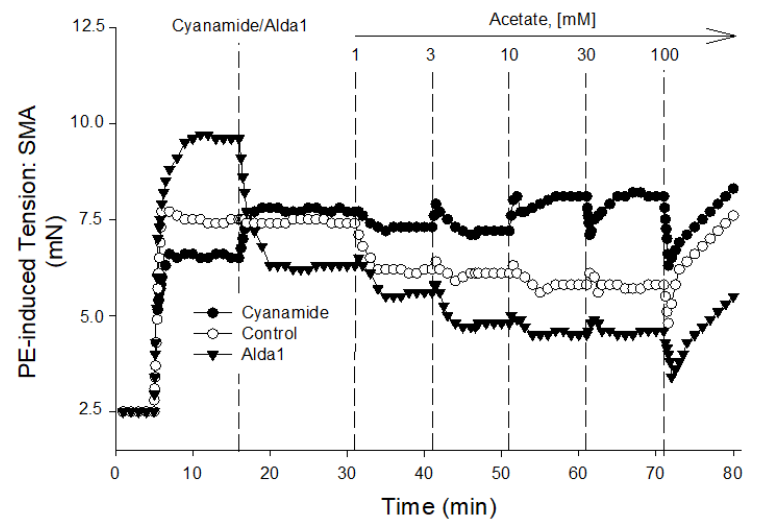

B.

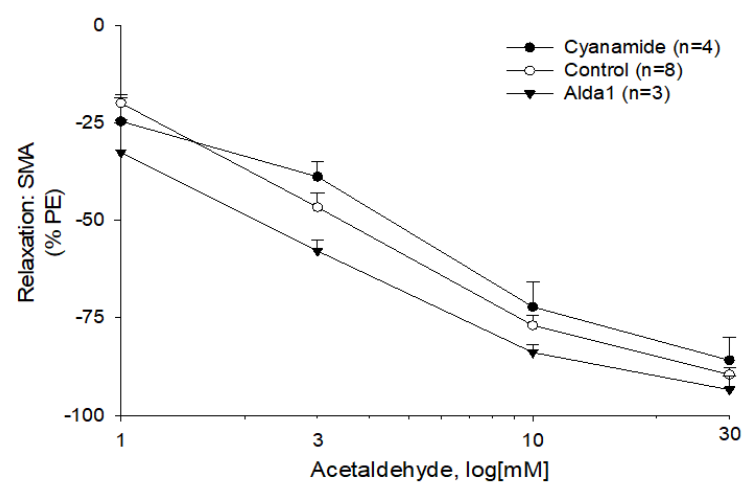

D.

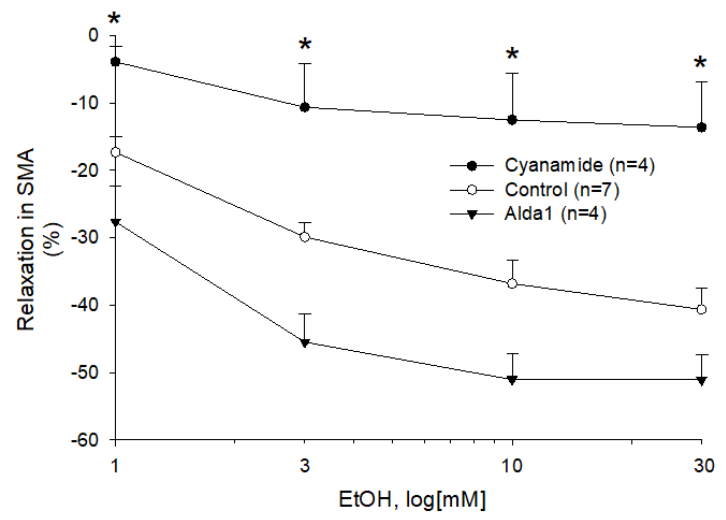

F.

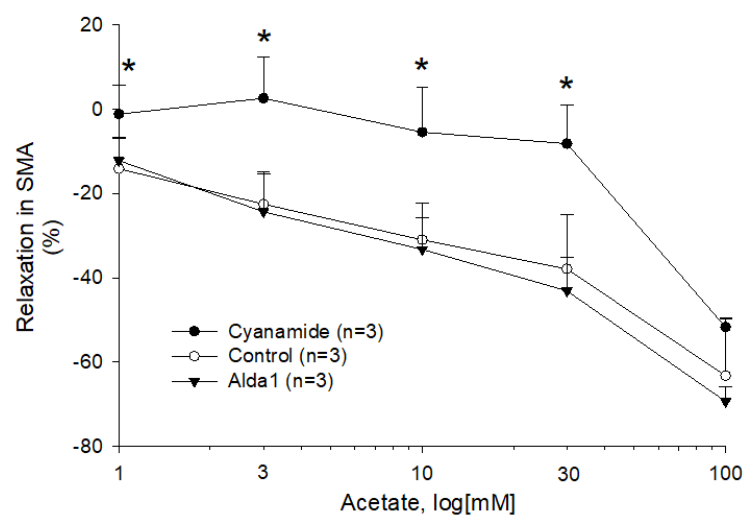


Fig. 22. Mechanisms of acetaldehyde-induced vasorelaxation and relationship to aldehyde metabolism. A) Cartoon depicting the mechanisms by which acetaldehyde (AA) induces vasorelaxation in SMA precontracted with phenylephrine (PE), thromboxane $A_{2}$ analog (U46,619) and $60 \mathrm{mM}$ potassium solution $\left(\mathrm{High} \mathrm{K}^{+}\right)$. AA induces a sensitive endothelial cell-dependent mechanism that begins with eNOS activation, NO formation, and GC activation leading to VSMC hyperpolarization and vasorelaxation (Pathway designated "1."). Several steps were confirmed by direct antagonism of NOS (L-NAME), endothelium (impaired by air perfusion) and guanylyl cyclase (GC; ODQ). A less sensitive yet equally efficacious pathway of vasorelaxation included a voltage-insensitive mechanism that similarly reversed $\mathrm{U} 46,619$ - and High $\mathrm{K}^{+}$-induced contraction implicating closure of VSMC Ca ${ }^{2+}$ channels (Pathway designated "2."). B) Cartoon depicting the metabolic pathways of ethanol, acetaldehyde and acetate. Ethanol is oxidized to acetaldehyde by alcohol dehydrogenase (ADH) and/or catalase activity. Acetaldehyde is oxidized to acetate by aldehyde dehydrogenases (ALDH1/ALDH2) or reduced back to ethanol by aldose reductase (AR). Acetate is converted to acetyl coenzyme A (CoA). 

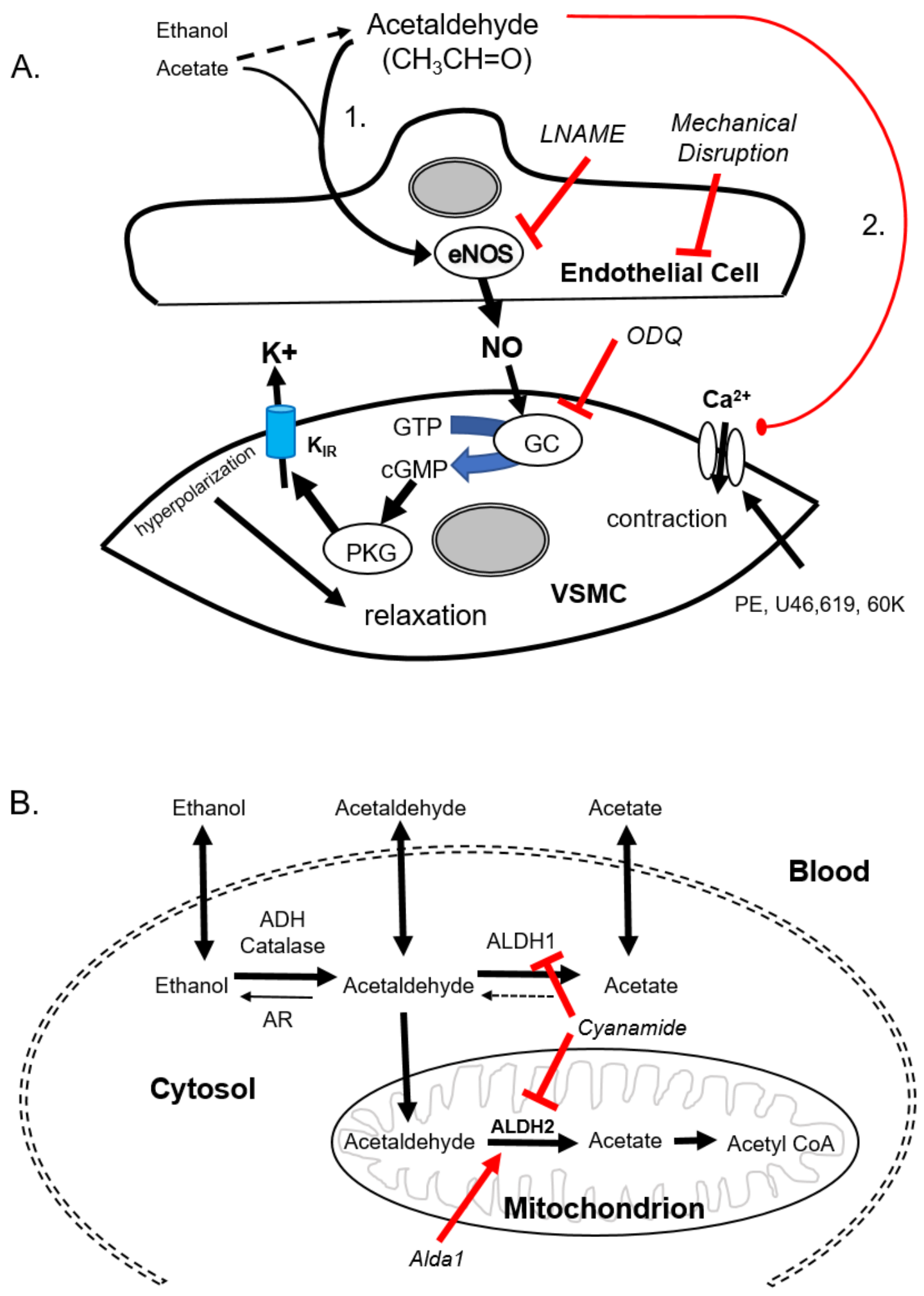
Table 13. Role of aldehyde dehydrogenase (ALDH) in AA-induced relaxations of PE-precontracted SMA. The efficacy (A; measured as the maximal relaxation, $\left.E_{\max } ; \%\right)$ and sensitivity (B; measured as the effective concentration inducing $50 \%$ relaxation; $\mathrm{EC}_{50}$; in $\mathrm{mM}$ ) or the of $\mathrm{AA}-$, $\mathrm{EtOH}^{-}$, or acetate-induced vasorelaxation in isolated murine superior mesenteric artery (SMA; male) pre-contracted with PE in the presence of cyanamide or Alda1 (ALDH2 activator).

\section{A. Efficacy}

\begin{tabular}{|c|c|c|c|}
\hline SMA & \multicolumn{3}{|c|}{$E_{\max }$ (\%Relaxation of PE-precontraction) } \\
\hline $\begin{array}{c}\text { Compound } \\
(\mathbf{1 - 3 0} \mathbf{~ m M})\end{array}$ & Control (vehicle) & +Cyanamide & +Alda1 \\
\hline Acetaldehyde & $89.5 \pm 4.8$ & $85.9 \pm 5.9$ & $93.4 \pm 3.5$ \\
\hline Ethanol & $40.6 \pm 3.1$ & $13.6 \pm 6.8^{*}$ & $52.2 \pm 4.4$ \\
\hline Acetate & $38.1 \pm 5.1$ & $8.2 \pm 9.1^{*}$ & $43.1 \pm 8.0$ \\
\hline
\end{tabular}

\section{B. Sensitivity}

\begin{tabular}{|c|c|c|c|}
\hline SMA & \multicolumn{3}{|c|}{ EC $_{50}$, [in mM] } \\
\hline $\begin{array}{c}\text { Compound } \\
(\mathbf{1 - 3 0} \mathbf{~ m M})\end{array}$ & Control (vehicle) & +Cyanamide & +Alda1 \\
\hline Acetaldehyde & $2.5 \pm 0.4$ & $3.7 \pm 0.7$ & $2.0 \pm 0.3$ \\
\hline Ethanol & $1.5 \pm 0.2$ & $X$ & $1.2 \pm 0.4$ \\
\hline Acetate & $2.4 \pm 0.8$ & $X$ & $3.6 \pm 0.9$ \\
\hline
\end{tabular}

Values $=$ means \pm SE. Abbr.: PE, phenylephrine, $10 \mu \mathrm{M} ; \mathrm{X}$, could not calculate due to low \% relaxation. Cyanamide, $1 \mathrm{mM}$; Alda1, $25 \mu \mathrm{M}$. N=3-6 mice per group. *, $\mathrm{P}<0.05$ vs Vehicle Control. 


\section{Discussion}

The physiological vascular action of AA is unclear but our study provides evidence for a potential contribution of AA in postprandial hyperemia in SMA via an EDRF-mediated dilatory pathway. In support, we show that SMA is significantly more sensitive than the aorta to AA, and importantly, the SMA is a known major arterial blood supply to the Gl tract that dilates postprandially in response of a variety of stimuli e.g., nutrients, vago-vagal reflex, NO, substance $\mathrm{P}$, etc... (Chou \& Coatney, 1994; Lucchini et al., 1996). Moreover, because rodents feed at night and sleep (fast) during the day, we observed a circadian difference in urinary acetate levels indicating endogenous AA level likely increases with feeding (see Table 9). Although, AA is oft considered an inhaled toxicant, its natural abundance in food, water and beverages (Aldehydes, 1981; D. J. Conklin, P. Haberzettl, et al., 2011) and as an abundant metabolic product, e.g., EtOH, make it a plausible stimulus for increasing Gl blood flow. As this is the first suggestion of this specific physiological role that we know of, more research will be needed to establish AA as a bona fide stimulus of postprandial hyperemia in vivo.

We found there are two distinct pathways of AA-induced vasorelaxation in SMA (Fig. 22A), and, we focused on the mechanism of the most sensitive pathway as it is likely a physiological pathway. The sensitive AA-induced vasorelaxation requires a functional endothelium, NOS (presumably eNOS) and guanylyl cyclase (GC/cGMP). The endothelial cells likely contribute to the most sensitive components, whereas the latter component is likely present in vascular smooth muscle cells (VSMC). These components are also activated by formaldehyde in 
rat aorta and murine SMA (Jin, Jagatheesan, et al., 2019; Q. Zhang et al., 2018), yet the TRPA1 channel is not stimulated by AA indicating a different 'activating step' in the endothelium for AA. Functionally, L-NAME, the eNOS inhibitor, significantly blocks the most sensitive component of the AA-induced relaxation and shifts the $\mathrm{EC}_{50}$ to the right. But this shift is less strong than that of endothelium disruption and ODQ (a GC inhibitor) suggesting a second endothelium-dependent component likely is operative. We also cannot rule out the possibility that additional components in the vascular wall (perivascular nerves, SubP, CGRP, etc...) may contribute to the AA-induced relaxation (Nilius et al., 2012).

AA induced a much stronger relaxation in SMA (and aorta) than either an upstream precursor, EtOH, or its downstream metabolite, acetate - both of which stimulate vasorelaxation in SMA but not in aorta indicating important similarities and differences with AA. EtOH-induced vasorelaxations have been welldocumented for over 4 decades, yet the complete mechanism of this phenomenon is only now becoming clearer. EtOH induces vascular relaxation by acting on both endothelial cells and VSMCs to dilate mesenteric and cutaneous blood vessels; yet EtOH also contracts cerebral, coronary, pulmonary and renal vascular beds and skeletal muscle arterioles (Altura \& Altura, 1982, 1987; Altura, Altura, \& Carella, 1983; Greenberg et al., 1993; Rocha et al., 2012; Ru et al., 2008; Toda, Konishi, Miyazaki, \& Komura, 1983). Our data show that EtOH (and acetate) relax SMA at concentrations $<100 \mathrm{mM}$ after which there is an onset of contraction. In contrast, $\mathrm{EtOH}$ and acetate only increase tension in precontracted aorta. Nonetheless, LNAME significantly blocks the most sensitive component of both $\mathrm{EtOH}$ - and 
acetate-induced relaxation in SMA reflecting NO-dependence (endothelium) -- as observed for AA (see shared Pathway 1. in Fig. 22A). Because the relaxations induced by $\mathrm{EtOH}$ and acetate in SMA are qualitatively similar to $\mathrm{AA}$, this may reflect a role of $\mathrm{AA}$ in both $\mathrm{EtOH}$ - and acetate-induced relaxations. As AA induces relaxation in both SMA and aorta this likely reflects a shared yet less sensitive pathway (\#2.; see Fig. 22A) that extracellular AA but not EtOH or acetate.

The concentration of AA in human blood is kept very low due to fast acting aldehyde dehydrogenase 2 (ALDH2) enzyme. The capacity of the liver to eliminate AA formed from $\mathrm{EtOH}$ is so efficient that measurable levels of $A A$ are typically undetected in peripheral blood (Lindros, Stowell, Pikkarainen, \& Salaspuro, 1980) but AA is detected in saliva. In an alcohol drinking model, salivary AA concentration was approximately $150 \mu \mathrm{M}$ (maximum $260 \mu \mathrm{M}$ ) over the first 20 to 40 min followed by a rapid decrease to 20 to $30 \mu \mathrm{M}$ for the remainder of the follow-up period (350 min)(Salaspuro, 2017). Moreover, ALDH2*2 enzyme deficiency is present in 35$45 \%$ of Asian people ( $8 \%$ of world's population), and results from $A L D H 2^{*} 2$ gene mutation that decreases ALDH activity by $20 \%$ (heterozygous mutation) and $50 \%$ (homozygous), and thus, people with $A L D H 2 * 2$ gene mutation are susceptible to facial flushing and nausea after alcohol consumption presumably due to high AA levels (Chen, Ferreira, Gross, \& Mochly-Rosen, 2014; Gross et al., 2015). Consistent with epidemiological findings, alcohol drinking in ALDH2-deficient subjects markedly elevates AA concentrations in saliva (Vakevainen, Tillonen, Agarwal, Srivastava, \& Salaspuro, 2000; Yokoyama et al., 2008) and in blood of EtOH- or AA-injected ALDH2-deficient mice (Jamal et al., 2016). Interestingly, high 
levels of AA are associated with increased risk of Gl tract cancers (Salaspuro, 2017) indicating that AA is likely elevated in the Gl tract as well.

Although concentrations of salivary and blood AA and those used in our study are difficult to compare, theoretically, consuming as little as one glass of wine (5 oz or $150 \mathrm{ml}$ ) containing $15-20 \mathrm{~g}$ of EtOH (11-13\% ABV) would increase blood EtOH concentration up to $65 \mathrm{mM}$ (assuming 5 I blood volume, $70 \mathrm{~kg}$ adult). As $\mathrm{EtOH}$ is rapidly metabolized to AA by $\mathrm{ADH}$ in liver and in blood vessels, it is plausible that AA levels increase quite quickly in endothelial cells and VSMC with corresponding intracellular/membrane levels being likely in the bioactive range described in our study (1-30 mM). Despite this theoretical calculation, there is little evidence for circulating AA levels in the active dilation range presumably because of rapid intracellular oxidative metabolism of AA by ALDHs to acetate (see Fig. 22B). To test the role of SMA-dependent ALDH metabolism on AA's bioactivity, we use an ALDH inhibitor, cyanamide, and an ALDH2 activator, alda-1, to decrease and increase ALDH activity, respectively, and in turn, potentially augment and diminish AA's action. Interestingly, alda-1 (25 $\mu \mathrm{M})$ and cyanamide (1 $\mathrm{mM}$ ) are vasoactive in SMA with opposing effects: alda-1 relaxed; cyanamide contracted (see Fig. 21E). Because of these opposing effects, we infer that the pharmacological target - (ALDH2 enzyme activity) may be an active regulator of SMA vascular tone. Perhaps more surprising, neither compound significantly alters AA-induced relaxation, yet cyanamide effectively blocks both $\mathrm{EtOH}$ - and acetateinduced relaxations in SMA. In contrast to our hypothesis, wherein we expect cyanamide (or AR-null) to enhance EtOH-induced relaxation by increasing $A A$ 
level, we observe inhibition of $\mathrm{EtOH}$-induced relaxation. Notably, this result is not without precedent. For example, EtOH combined with cyanamide inhibits rather than enhances EtOH-induced increases in blood flow (Carmichael et al., 1987). Interestingly, AA alone did not increase portal blood flow either. Equally provocative is our finding that alda- 1 increases the relaxation of $\mathrm{EtOH}$ but does not alter relaxation of either AA or acetate. Collectively, these data are both compelling (ALDH activity likely is involved in vascular effects of EtOH and acetate.) and vexing (Why are vascular effects of $A A$ unaltered by modulators of ALDH activity?). The latter may be a function of addition of extracellular addition of AA whereas both $\mathrm{EtOH}$ and acetate need to be intracellular in order to be metabolized to bioactive AA. The cyanamide-mediated inhibition of $\mathrm{EtOH}$ - and acetate-induced relaxations further indicates dependence on ALDH activity for initiation of relaxation. There is, of course, precedent for such a role of ALDH2 activity in nitroglycerin-induced vasorelaxation (Beretta et al., 2012; Hink et al., 2007), however, others show that cyanamide alone induces relaxation in isolated and precontracted rabbit aorta (Fukuto, Gulati, \& Nagasawa, 1994). Moreover, as nicotine did not alter AA-induced SMA relaxation, we infer nicotine does not inhibit vascular ALDH activity. To dissect out the complex biochemical interplay between AA vascular action and ALDH activity will require further studies.

In conclusion, we describe a sensitive component of an AA-induced vasorelaxation in SMA that is sequentially dependent on an EDRF/NO and a GC/cGMP pathway. We suggest this relaxation is a likely contributor to a broader, multifaceted postprandial hyperemic reflex that increases blood flow to the Gl tract 
to augment digestion and nutrient absorption. Moreover, EtOH and/or acetate (a short chain fatty acid metabolite of gut microbiota) may contribute to local vascular AA levels (via ADH and ALDH activities) to further augment the reflex increase in gut blood flow related to feeding (and drinking). 


\section{CHAPTER V}

CROTONALDEHYDE-INDUCED VASCULAR RELAXATION AND TOXICITY:

ROLE OF ENDOTHELIUM AND TRANSIENT RECEPTOR POTENYTIAL

ANKYRINE-1 (TRPA1)

Introduction: Crotonaldehyde $(\mathrm{CR})$ is an electrophilic $\alpha, \beta$-unsaturated aldehyde present in foods and beverages, and as a minor metabolite of 1,3butadiene. CR is a product of incomplete combustion, and thus, it is measured at relatively high levels (ppm) in cigarette smoke and structural fires. Exposure to $\mathrm{CR}$ has been linked to cardiopulmonary toxicity and cardiovascular disease including atherosclerosis. Objective: The purpose of this study was to examine the direct effects of CR in isolated murine blood vessels (aorta and superior mesenteric artery, SMA) in vitro, and dissect the underlying mechanisms. Method and Results: CR induced concentration-dependent (1-300 $\mu \mathrm{M})$ and equally robust relaxations (75-80\%) in phenylephrine (PE) precontracted aorta and SMA, yet the SMA was 20x more sensitive than aorta to CR (SMA EC50 3.8 $\pm 0.5 \mu \mathrm{M}$; aorta EC50 $76.0 \pm 2.0 \mu \mathrm{M} ; \mathrm{p}<0.05)$. To probe the mechanisms of $\mathrm{CR}$-induced relaxation and toxicity, we used the sensitive SMA. The CR-induced relaxation was inhibited in 
the presence of: 1) mechanically-impaired endothelium; 2) N $\omega$-Nitro-L-arginine methyl ester hydrochloride (L-NAME); 3) guanylyl cyclase (GC) inhibitor (ODQ); 4) transient receptor potential ankyrin-1 (TRPA1) antagonist (A967079); and, 5) nonvasoactive level of nicotine $(1 \mu \mathrm{M})$. Similarly, a known TRPA1 agonist, allyl isothiocyanate (AITC; mustard oil) also stimulated SMA relaxation dependent on: endothelium, TRPA1, NO, and GC. Moreover, TRPA1 was co-localized with isolectin by positive immunofluorescent staining of the SMA endothelium. Thus, CR (1-30 $\mu \mathrm{M})$ induces relaxation in SMA via an endothelium-, NO-, GC-, and TRPA1-dependent pathway. Additionally, CR was toxic at high concentrations (100-300 $\mu \mathrm{M})$ where it stimulated tension oscillations and significantly depressed subsequent PE-induced contractions after washout. CR-induced vasotoxicity was significantly enhanced in SMA with impaired endothelial function. Conclusions: The sensitive relaxation of CR depends on a functional endothelium and TRPA1 whereas vasotoxicity was enhanced under conditions of endothelium dysfunction. $\mathrm{CR}$ is both vasoactive and vasotoxic along a concentration continuum with elevated CR likely encountered in smoke exposures.

\section{Introduction}

Crotonaldehyde $(C R)$ is a highly reactive $\alpha, \beta$-unsaturated aldehyde that is environmentally ubiquitous, e.g., found in foods such as fish, meat, fruits and vegetables and beverages such as wine and whiskey (Eder, Schuler, \& Budiawan, 1999; IARC., 1995). Sources of CR exposure are mainstream and side-stream tobacco smoke (IARC., 1995). CR is also formed endogenously during lipid 
peroxidation (Chung, Chen, \& Nath, 1996; Chung et al., 1999; Duescher \& Elfarra, 1993; Filser et al., 2001; Kawaguchi-Niida et al., 2006; Nair, Bartsch, \& Nair, 2007; Voulgaridou, Anestopoulos, Franco, Panayiotidis, \& Pappa, 2011) and in the metabolism of 1,3-butadiene (Duescher \& Elfarra, 1993; Filser et al., 2001) and Nnitrosopyrrolidine (Hecht, Upadhyaya, \& Wang, 1999; M. Y. Wang, Chung, \& Hecht, 1988). CR can also be formed from acetaldehyde by aldol condensation and dehydration (Tuma, Hoffman, \& Sorrell, 1991). CR is an important industrial chemical used in the synthesis of tocopherols, sorbitol, and 3-methylbutanol, but it is also a contaminant and a byproduct of various chemical processes (IARC., 1995).

CR can rapidly penetrate cell membranes and induce adverse biological effects, such as inflammation in alveolar macrophages of smokers (Facchinetti et al., 2007; Yang et al., 2013a; Yang et al., 2013b); oxidative stress and apoptosis in epithelial cells (Liu, Yang, Pan, Zhu, \& Xie, 2010a, 2010b); and toxicity to respiratory tract tissues (Reddy et al., 2002). Glutathione S-transferase P (GSTP) accelerates metabolism of short chain unsaturated aldehydes such as acrolein (3C) and $\mathrm{CR}(4-\mathrm{C})$ via conjugation with glutathione (GSH) (Berhane, Widersten, Engstrom, Kozarich, \& Mannervik, 1994; D. J. Conklin, Haberzettl, et al., 2015a). The major metabolite of CR and a urinary biomarker of CR exposure is 3-hydroxy1-methylpropyl mercapturic acid (HPMMA) (Gray \& Barnsley, 1971). Urinary levels of HPMMA are significantly increased after cigarette smoking and are significantly higher (3x) in U.S. cigarette smokers than in non-smokers (Jain, 2015; Lorkiewicz et al., 2019). Moreover, levels of both HPMMA and 3HPMA (major metabolite of 
acrolein) are similar in smokers yet as well as in non-smokers indicating similar levels of these two aldehydes are found in the environment, derived from endogenous metabolic processes and in cigarette smoke (Jain, 2015).

$\mathrm{CR}$ is chemically similar to acrolein, and thus, the lung toxicity of cigarette smoke has been attributed in part to both acrolein and CR. It has been listed as one of nine constituents recommended for disclosure and monitoring by the World Health Organization (WHO) study group on tobacco product regulation (Haussmann, 2012). Moreover, because the primary acrolein metabolite, 3HPMA, is associated with cardiovascular and pulmonary disease risk (DeJarnett et al., 2014; Srivastava et al., 2011; Zhang et al., 2018), it is expected that CR also contributes to disease risk. Similarly, occupational exposure to 1,3-butadiene, a precursor of CR, is associated with cardiovascular disease, and in an animal model, exposure to 1,3-butadiene increases arteriosclerotic plaque development in cockerels (Penn \& Snyder, 1996b). Nonetheless, little research has been dedicated to assess the direct vascular actions of CR.

Because $\mathrm{CR}$ and acrolein are known to activate the transient receptor potential ankyrin 1 (TRPA1) channel and because TRPA1 is present in the vasculature, we explored the TRPA1-dependence of CR vasoreactivity in isolated blood vessels. TRPA1 is widely distributed in the nervous and cardiovascular systems especially sensory neurons (unmyelinated C-fibers) (Anand et al., 2008; Nagata, Duggan, Kumar, \& Garcia-Anoveros, 2005), endothelial cells (Earley et al., 2009a), and heart and cardiomyocytes (Andrei, Sinharoy, Bratz, \& Damron, 2016; D. J. Conklin, Y. Guo, et al., 2019). Activation of TRPA1 by a variety of 
agonists including acrolein, CR and allyl isothiocyanate (AITC, mustard oil) leads to pain (Trevisan et al., 2016), neurogenic tissue inflammation (Schwartz et al., 2011), pulmonary edema and airway hyper-reactivity (Andre et al., 2008; D. J. Conklin, Haberzettl, Jagatheesan, Kong, \& Hoyle, 2016), vasodilation (Jin, Jagatheesan, et al., 2019) and hypotension (Earley, 2012; Pozsgai et al., 2010). Thus, TRPA1 could be important in the vasoactive effects of CR.

Consistent with our hypothesis, our research showed that CR induced a robust, concentration-dependent relaxation that in superior mesenteric artery (SMA) was dependent on: 1) a functional endothelium; 2) NO formation; 3) guanylyl cyclase (GC); and, 4) TRPA1 activation. High concentrations of CR, however, led to tension oscillations and irreversible suppression of subsequent contractility reflecting acute vasotoxicity. These data indicate that $C R$ is vasoactive with TRPA1 at low concentrations and vasotoxic at high concentrations.

\section{Material and Methods}

\section{Chemicals and Solutions.}

Reagent grade chemicals were purchased from Sigma-Aldrich or other commercial sources as indicated: A967079 (AdooQ); acetylcholine chloride (ACh); crotonaldehyde (CR); allyl isothiocyanate (AITC); 1h-[1,2,4]oxadiazolo[4,3a]quinoxalin-1-one (ODQ); $\mathrm{N}^{\omega}{ }^{\omega}$-nitro-L-arginine methyl ester hydrochloride (LNAME); L-phenylephrine hydrochloride (PE); sodium nitroprusside (SNP).

\section{Animals.}


Male or female wild type C57BL/6J (WT), TRPA1-null and GSTP-null mice (12-20 weeks old; 25-35g) were purchased (The Jackson Laboratory, Bar Harbor, ME) or used from in house breeding (D. J Conklin et al., 2017; D. J. Conklin, Haberzettl, et al., 2009b). Mice were treated according to American Physiological Society Guiding Principles in the Care and Use of Animals, and all protocols were approved by University of Louisville Institutional Animal Care and Use Committee. Mice were housed under pathogen-free conditions in the University of Louisville vivarium under controlled temperature and $12 \mathrm{~h}$ light:12h dark cycle. Mice were provided water and a standard chow diet ad libitum (Rodent Diet 5010, 4.5\% fat by weight, LabDiet; St. Louis, MO).

\section{Urine Collection and CR Metabolite Analysis}

Urine Collection. To measure total urine HPMMA, mice were exposed to HEPAfiltered air or mainstream cigarette smoke (MCS; KY Reference 3R4F cigarettes: $50 \%$ of smoke of 12 cigarettes over $6 \mathrm{~h}$ ) and then placed singly per metabolic cage with glucose/saccharin solution (w/v; 3.0\%/0.125\%) to collect urine (without food) up to 3h post-exposure followed by urine collection overnight $(\mathrm{O} / \mathrm{N}$; with food). Urine samples were centrifuged in cold immediately after collection $(600 \times g, 5 \mathrm{~min}$; to pellet any feces and food particles), decanted, and stored at $-80^{\circ} \mathrm{C}$ (D. J. Conklin et al., 2018).

CR Metabolite Analysis. The major urine metabolite of CR, 3-hydroxy-1methylpropylmercapturic acid (HPMMA), was quantified by ultrafast performance liquid chromatography-mass spectrometry (UPLC-MS) as described (D. J. Conklin et al., 2018). Briefly, urine $(25 \mu \mathrm{l})$ was mixed with $15 \mathrm{mM}$ ammonium acetate (975 
$\mu$ l) containing HPMMA-d3 standard (667 ng) and filtered through a $0.2 \mathrm{~mm}$ PTFE membrane. Samples $(2 \mu \mathrm{l})$ were applied on UPLC and the eluate was analyzed for the MRM traces by MS. To account for urinary dilution, all values for urinary metabolites were normalized to urinary creatinine (mg/dl) (D. J. Conklin et al., 2018).

CR-Induced Vasoreactivity in Isolated Aorta and Superior Mesenteric Artery (SMA) Isolation and Organ Bath Conditions. After removal and cleaning of the aorta and SMA, thoracic aorta rings (3-4 $\mathrm{mm})$ were hung on stainless steel hooks in organ baths and SMA rings $(2 \mathrm{~mm})$ were hung on tungsten wire in $5-\mathrm{ml}$ heated organ baths (MultiWire Myograph System 620M, DMT, Denmark) in PSS bubbled with $95 \% \mathrm{O}_{2}: 5 \% \mathrm{CO}_{2}$ at $37^{\circ} \mathrm{C}$. After 10 min without tension, aorta and SMA rings were equilibrated to respective loading tension over $30 \mathrm{~min}$ (aorta, $1 \mathrm{~g}$; SMA, $0.25 \mathrm{~g}$ ). All rings were stimulated with $\mathrm{High} \mathrm{K}^{+}(60 \mathrm{mM})$ to test for viability, washed 3 times with PSS over $30 \mathrm{~min}$, and re-equilibrated to appropriate resting tension (Jin, Jagatheesan, et al., 2019). Krebs physiological salt solution (PSS) for SMA was (in $\mathrm{mM}$ ): $\mathrm{NaCl}, 119 ; \mathrm{KCl}, 4.7 ; \mathrm{CaCl}_{2}, 2 ; \mathrm{MgCl}_{2}, 1.2 ; \mathrm{KH}_{2} \mathrm{PO}_{4}, 1.2 ; \mathrm{NaHCO}_{3}, 24$; glucose, 7; $\mathrm{pH}$ 7.4. Krebs PSS for aorta was (in $\mathrm{mM}$ ): $\mathrm{NaCl}, 119 ; \mathrm{KCl}, 4.7 ; \mathrm{CaCl}_{2}$, 1.2; $\mathrm{KH}_{2} \mathrm{PO}_{4}, 1.2 ; \mathrm{MgSO}_{4}, 1.2 ; \mathrm{NaHCO}_{3}, 25 ;$ glucose, 5.5; $\mathrm{pH}$ 7.4. High K+ PSS (High $\mathrm{K}^{+} ; 60 \mathrm{mM}$ ) substituted equimolar $\mathrm{K}^{+}$for $\mathrm{Na}^{+}$in SMA PSS.

Crotonaldehyde (CR) Experimental Series:

CR-Induced Relaxation. Crotonaldehyde $(1,3,10,30,100$, and $300 \mu \mathrm{M})$ was added to organ bath containing uncontracted and pre-contracted (PE, $\left.10 \mu \mathrm{M} ; \mathrm{PE}_{1}\right)$ aorta and SMA. Following exposure to the highest concentration of $\mathrm{CR}$, organ 
baths were washed $(3 \mathrm{x})$ with fresh PSS over $30 \mathrm{~min}$, and then vessels were precontracted again with $\mathrm{PE}\left(\mathrm{PE}_{2}\right)$ and relaxed sequentially with $\mathrm{ACh}(10 \mu \mathrm{M})$ and then SNP $(100 \mu \mathrm{M})$ to test for endothelium-dependent and -independent relaxations, respectively. The efficacy of CR-induced relaxation was calculated as the $\%$ reduction in agonist-induced contraction. The sensitivity of CR-induced relaxation was the effective concentration producing $50 \%$ response $\left(E_{50}\right)$, i.e., cumulative concentration responses normalized to $100 \%$ with interpolation of $\mathrm{EC}_{50}$ (Jin, Jagatheesan, et al., 2019). EC 50 values were calculated only if total relaxation \% exceeded $50 \%$ of $\mathrm{PE}_{1}$. Contractile toxicity was quantified as the $\mathrm{PE}_{2} / \mathrm{PE}_{1}$ tension ratio (as \%) by dividing PE-induced tension post-CR-exposure $\left(\mathrm{PE}_{2}\right)$ by $\mathrm{PE}-$ induced tension pre-CR (i.e., $\mathrm{PE}_{1}$ ) multiplied by 100 . Tension oscillations were quantified by their frequency (oscillations/min) and their amplitude $(\mathrm{mN})$ as averaged after high CR concentration exposure $(300 \mu \mathrm{M})(\mathrm{D}$. J. Conklin, Boyce, Trent, \& Boor, 2001a).

Role of the Endothelium, Nitric Oxide Synthase (NOS), and cGMP. The role of the endothelium in CR-induced vasorelaxation was studied in SMA with intact and injured endothelium. The endothelium was mechanically injured by air perfusion and damage confirmed by near complete abolition (>95\%) of ACh-induced dilation of PE pre-contracted SMA (Jin, Jagatheesan, et al., 2019). To evaluate the role of NOS, L-NAME $(100 \mu \mathrm{M})$ was added after addition of PE (10 $\mu \mathrm{M} ; 15 \mathrm{~min})$, and, precontracted SMA were then relaxed with cumulative concentrations of $C R(1,3,10$, 30, 100 and $300 \mu \mathrm{M})$. To assess whether cGMP was involved in CR-induced 
vasorelaxation, PE-precontracted SMA were exposed to ODQ $(3 \mu \mathrm{M})$ to inhibit guanylyl cyclase (GC) and subsequent formation of cGMP (Jiang et al., 2015).

Role of TRPA1. The role of the TRPA1 channel was assessed by incubation of SMA with TRPA1 antagonist (A967079, $1 \mu \mathrm{M})$ followed by cumulative addition of CR (1-300 $\mu \mathrm{M})$. Similarly, effects of CR in SMA of TRPA1-null mice were measured without and with the TRPA1 antagonist (A967079, $1 \mu \mathrm{M})$ present. Likewise, effects of a known TRPA1 agonist, allyl isothiocyanate (AITC, 1-300 $\mu \mathrm{M}$ ), were measured in PE-precontracted SMA.

Role of GSTP on CR Relaxation. Because CR is primarily metabolized by glutathione S-transferase P (Berhane et al., 1994), we assessed whether GSTP deficiency could enhance effects of CR in isolated blood vessels of GSTP-null mice. Blood vessels were PE-precontracted and exposed to increasing concentrations of $\mathrm{CR}(1-300 \mu \mathrm{M})$ as described above.

Role of Nicotine. Because exposure to $\mathrm{CR}$ via tobacco smoke also will necessarily contain nicotine, we tested whether a super-pharmacological level of nicotine (1 $\mu \mathrm{M})$ would affect CR-induced relaxation in PE-precontracted SMA.

Histology and TRPA1 Immunofluorescence. Thin sections (4 $\mu \mathrm{m})$ of formalin-fixed, paraffin-embedded SMA, aorta and dorsal root ganglia (DRG; positive control) of WT mice were stained with H\&E or TRPA1 antibody (1:200; Alomone Labs, Israel; Cat. \#: ACC-037). To label endothelial cells, blood vessel sections were co-stained with isolectin GS-IB4 Alexa Fluor 594 conjugate (1:200 dilution; Invitrogen; Cat. \#: I21413). Images of H\&E-stained sections were made using a digital Spot camera 
mounted on an Olympus microscope. Immunofluorescence microscopy was performed with rabbit polyclonal antibody against TRPA1 without or with a blocking peptide of the TRPA1 antibody and Alexa Fluor 647 goat anti-rabbit secondary antibody (1:400 dilution; Invitrogen; Cat. \#: 21244). Slides were covered with DAPI containing Slow Fade ${ }^{\circledR}$ Gold anti-fade reagent (Invitrogen; Cat. \#: S36938), and fluorescence was visualized on a Nikon eclipse Ti fluorescence Microscope using NIS-Elements (Nikon; Japan) at 200X. A DAPI filter was used for nuclear staining and a Cy5 Red filter for TRPA1 staining (D. J. Conklin, Y. Guo, et al., 2019; Jin, Jagatheesan, et al., 2019).

Statistical Analyses. Data are expressed as means \pm SE. When comparing two groups, a $t$-test was used (paired or unpaired as appropriate). Multiple group testing was done with One-Way ANOVA with Bonferroni post hoc. If either equal variance or normality test failed, One-Way ANOVA on Ranks (Kruskal-Wallis) and Dunn's post hoc was used (SigmaPlot, ver. 12). Statistical significance was assumed where $\mathrm{P}<0.05$.

\section{Results}

Urinary CR Metabolite, HPMMA, is Elevated in Mice Exposed to Mainstream Cigarette Smoke (MCS).

The total urinary HPMMA $(\mu \mathrm{g})$ excreted from C57BL/6 (WT) mice exposed to KY Reference cigarette MCS (3R4F) was increased 2.5x over that in urine of air-exposed mice (Fig. 23A). As expected, urinary nicotine level also was 
increased significantly after MCS exposure (Fig. 23A). Baseline excretion of HPMMA (about $1.8 \mu \mathrm{g} / 16 \mathrm{~h}$ ) is equivalent to $0.025 \mu \mathrm{mol} \mathrm{CR}(\mathrm{MW}=70.07)$, and as blood volume of a $25 \mathrm{~g}$ mouse is about $2 \mathrm{ml}$, this amount of $\mathrm{CR}$ approximates to $12.5 \mu \mathrm{M}$ in blood. Moreover, as the HPMMA level was increased 2.5x after MCS exposure, a potential blood level of $\mathrm{CR}>30 \mu \mathrm{M}$ was estimated. So, we tested for direct vascular effects of $\mathrm{CR}$ from 1-300 $\mu \mathrm{M}$ to cover a broad systemic concentration range.

Concentration-Dependent Effects.

CR $(1-300 \mu \mathrm{M})$ produced robust (around 75-80\%) concentration-dependent relaxations in precontracted aorta and SMA (Fig. 23B-C), but relaxations did not reach $100 \%$ in SMA due to a consistent reversal of relaxation and induction of tension oscillations ("spasms") at $300 \mu \mathrm{M}$ (Fig. 23B). CR induced equally robust vasorelaxation in both isolated aorta and SMA, but the SMA was significantly more sensitive (20x) than aorta to CR (SMA EC $503.8 \pm 0.5 \mu \mathrm{M}$; aorta $\mathrm{EC}_{50} 76.0 \pm 2.0 \mu \mathrm{M}$; p<0.05) (Fig. 23D; Tables 14-15). Tension oscillation frequencies were low $(<2 / \mathrm{min})$ and of modest amplitude $(<2.5 \mathrm{mN})$ (Fig. 23B; Table 16). Endothelial impairment abolished CR induction of oscillations, and L-NAME treatment decreased oscillation frequency by $35 \%$ and amplitude by $60 \%$ although neither reduction was statistically significant (Table 16$)$.

CR $(1-300 \mu \mathrm{M})$ added to uncontracted SMA had no effect on basal tension (myogenic tone; data not shown) nor on $\mathrm{PE}_{2}$-generated tension $(84.0 \pm 12.8 \%$ of $\left.\mathrm{PE}_{1} ; \mathrm{n}=3\right)$ nor on $\mathrm{ACh}$-induced relaxation $(-91.1 \pm 1.3 \% ; n=3)$ in the continued 
presence of CR. However, after 3 bath exchanges over 30 min, CR-treated SMA were largely refractory to $\mathrm{PE}_{3}$-induced tension by $74.4 \pm 15.0 \%$ of $P E_{1}(n=3$; $P=0.0666)$; yet, retained functional $A C h-i n d u c e d$ relaxation $(-84.6 \pm 2.4 \% ; n=3)$. Mechanisms of CR-Induced Vasorelaxation.

The most sensitive component of CR-induced vasorelaxation $(1-30 \mu \mathrm{M})$ was significantly inhibited in PE-pre-contracted SMA by a mechanically disrupted endothelium (>90\% loss of ACh-induced relaxation) (Fig. 24A-B; Tables 14-15). Similarly, CR-induced vasorelaxation in pre-contracted SMA was equally inhibited in the presence of L-NAME, and the $\mathrm{EC}_{50}$ shifted rightward (less sensitive) (Fig. 24C-D; Tables 14-15). This L-NAME effect indicated a role of NO (likely eNOS) as critical in the most sensitive part of the CR-induced relaxation in PEprecontracted SMA. Similarly, ODQ treatment, an irreversible guanylyl cyclase (GC) inhibitor, significantly blocked only the most sensitive part of the CR-induced relaxation (Fig. 24E-F; Tables 14-15). Collectively, these data show that the sensitive portion of the CR-induced relaxation was dependent on the endothelium, NOS (likely eNOS) and NO-mediated activation of GC (and formation of cGMP in VSMC). The female SMA was marginally less sensitive to CR than male SMA (Table 15).

Role of TRPA1 channel in the CR-induced relaxation.

Because CR is a known agonist of TRPA1, we hypothesized that the TRPA1 channel contributes to endothelium-dependent CR-induced vasorelaxation in SMA. In support, the TRPA1 antagonist, A967079 $(1 \mu \mathrm{M})$, significantly inhibited 
the most sensitive part $(<10 \mu \mathrm{M})$ of the $\mathrm{CR}$-induced relaxation in PE-contracted SMA (Fig. 25A-B; Tables 14-15). We also tested CR-induced relaxation with or without A967079 $(1 \mu \mathrm{M})$ in PE-contracted SMA of male TRPA1-null mice (Fig. 25C-D; Tables 14-15). In contrast, presence of A967079 appeared to enhance slightly the relaxation of CR in TRPA1-null SMA (Fig. 25D). Because CR is a substrate of GSTP, we tested for a metabolic role of GSTP in SMA treated with $\mathrm{CR}$. There was no difference in the CR-induced relaxation in PE-contracted SMA of GSTP-null mice compared with WT SMA (Fig. 25E-F; Tables 14-15), and there was no difference in the CR-induced relaxation with or without ezatiostat which was a GSTP inhibitor (Fig. 25G-H; Tables 14-15). Because AITC is a known TRPA1 agonist, it, unsurprisingly, induced a concentration- and TRPA1-dependent relaxation in SMA (Fig. 26A). This relaxation, as with CR, was inhibited by the TRPA1 antagonist (A976709), and the AITC-induced relaxation was similarly shifted rightward in SMA of TRPA1-null mice (Fig. 26B), supporting the idea that TRPA1 mediates this sensitive vasorelaxation (see $\mathrm{EC}_{50}$ in Table 15).

In immunofluorescent-stained cross-sections of mouse SMA, TRPA1 (green; Fig. 26D) was co-localized with isolectin (red) in the endothelium (see yellow in Fig. 26E). Modest yet specific TRPA1 staining also was present in the media:adventitia border of SMA (green; Fig. 26D). Although aorta (Fig. 26F-H) had positive TRPA1 staining localized in the media (green; Fig. 26G), there was no staining observed in the endothelium. Positive TRPA1 staining was confirmed by use of a TRPA1 blocking peptide in aorta (Fig. 26H) and in dorsal root ganglion 
(DRG; Fig. 27), which also served as a positive control for TRPA1 (D. J. Conklin, Y. Guo, et al., 2019).

Vascular smooth muscle toxicity of CR.

The $\mathrm{PE}_{2} / \mathrm{PE}_{1}$ tension (\%) was significantly decreased after $\mathrm{CR}$ exposure (CR $\left.\mathrm{PE}_{2} / \mathrm{PE}_{1}: 27.0 \pm 2.2 \%\right)$ compared with the untreated control group $\left(\mathrm{PE}_{2} / \mathrm{PE}_{1}\right.$ : 133.6 $\pm 12.3 \%$ ) (Fig. 28A, Table 17). The $\mathrm{PE}_{2} / \mathrm{PE}_{1}$ tension (\%) was further decreased in endothelial dysfunction (ED) and ODQ treatment groups compared with the CR only exposed group (Fig. 28A) but there were no differences with any other treatment group. Although $\mathrm{CR}$ treatment alone depressed $\mathrm{PE}_{2}$ contraction, it had no effect on subsequent ACh-induced relaxation (Fig. 28B, Table 16). In fact, the only 2 groups with impaired ACh-induced relaxation following CR exposure were by design: ED and L-NAME treatments (Fig. 28B, Table 17) suggesting that CR did not induce acute endothelial cell injury despite causing VSMC dysfunction.

Nicotine and $C R$.

In users of combustible tobacco, CR and nicotine exposure occur simultaneously; therefore, we tested whether nicotine altered CR-induced relaxation in SMA. Nicotine (>10 $\mu \mathrm{M})$ induced robust relaxation in SMA (Fig. 29A). Nicotine alone $(1 \mu \mathrm{M})$ had no effect on tension in PE-contracted SMA, yet decreased slightly and significantly efficacy of subsequent CR-induced vasorelaxation (Table 14; Fig. 29B). 
Fig. 23. Crotonaldehyde-induced (CR) relaxation in aorta and superior mesenteric artery (SMA). A) Cumulative quantification of mass spectrometry analyses of primary crotonaldehyde (CR) metabolite (3-hydroxy-1methylpropylmercapturic acid; HPMMA) and nicotine in urine of mainstream cigarette smoke-exposed (MCS; 12 cigarettes/6 h) male mice 16h post-exposure. (Graph derived from data in Conklin et al., 2018). B) Representative trace of CRstimulated (1-300 $\mu \mathrm{M})$ relaxation and tension oscillations of phenylephrine (PE)contracted SMA, and post-CR exposure vasoreactivity. C) Representative trace of CR-stimulated (1-300 $\mu \mathrm{M})$ relaxation of phenylephrine (PE)-contracted aorta, and post-CR exposure toxicity (no contraction to PE). D) Summary sensitivity data of $\mathrm{CR}$-induced relaxation in PE pre-contracted SMA and aorta. Values $=$ means $\pm \mathrm{SE}$ of 3-4 preparations. ${ }^{*}, P<0.05$ vs aorta. 
A.

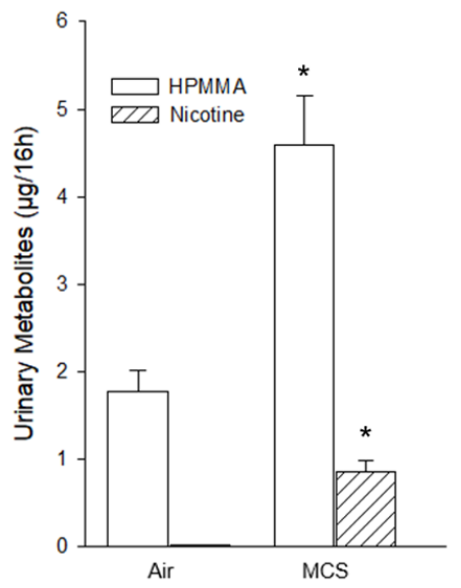

C.

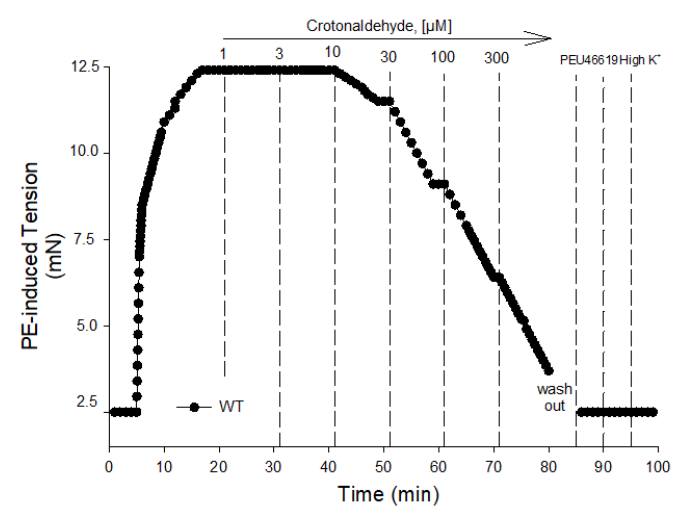

B.

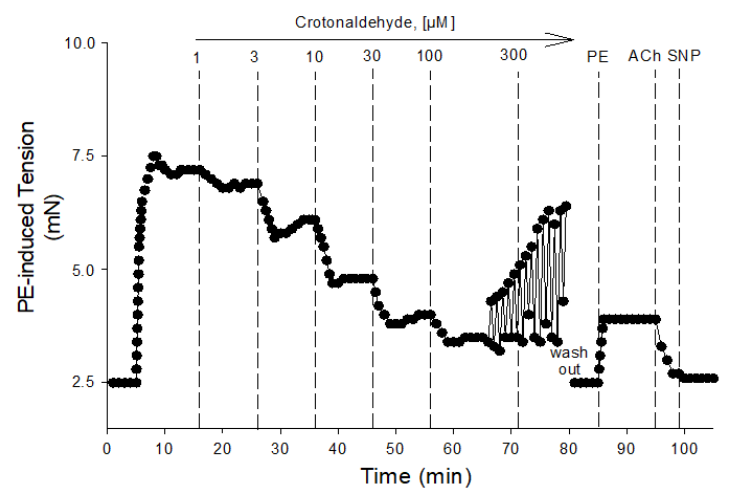

D.

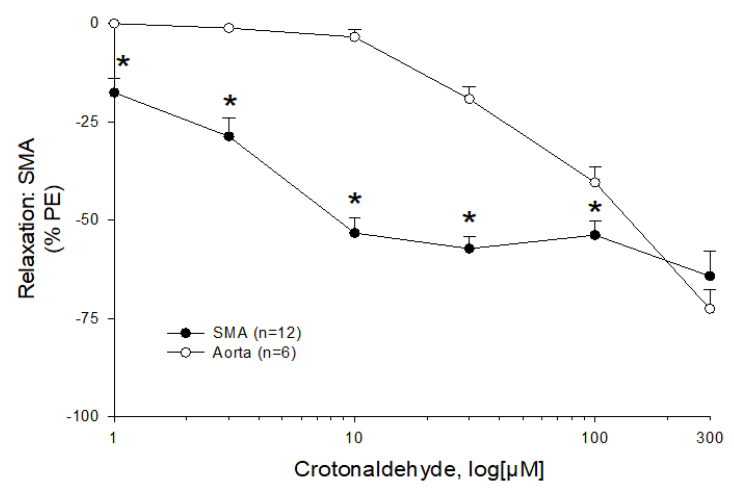


Table 14. Efficacy (maximal relaxation, $E_{\max }$ ) of crotonaldehyde-induced relaxations of PE-precontracted superior mesenteric artery (SMA; male and female) without and with inhibitor treatments.

\begin{tabular}{|c|c|c|}
\hline $\begin{array}{c}\text { SMA } \\
\text { (+treatment, } \mathbf{N} \text { ) }\end{array}$ & $\begin{array}{c}\mathbf{E}_{\max } \\
\text { (Control, PE+vehicle) }\end{array}$ & $\begin{array}{c}\mathbf{E}_{\max } \\
\text { (ED or PE+inhibitor) }\end{array}$ \\
\hline WT+ED & $-74.3 \pm 6.6$ & $-63.6 \pm 7.1$ \\
\hline WT+L-NAME & $-62.7 \pm 5.3$ & $-66.8 \pm 2.4$ \\
\hline WT+ODQ & $-73.4 \pm 6.9$ & $-54.7 \pm 15.2$ \\
\hline WT+A967079 & $-57.2 \pm 3.5$ & $-61.6 \pm 6.8$ \\
\hline TRPA1-null+A967079 & $-70.5 \pm 6.4$ & $-79.0 \pm 4.2$ \\
\hline WT+Ezatiostat & $-56.5 \pm 7.2$ & $-61.5 \pm 2.5$ \\
\hline GSTP-null & $-52.6 \pm 2.9$ & -- \\
\hline WT+Nicotine & $-67.0 \pm 4.2$ & $-55.0 \pm 6.5$ \\
\hline WT (f) & $-59.8 \pm 3.9$ & -- \\
\hline
\end{tabular}

Values $=$ means \pm SE. Abbr.: PE, phenylephrine, $10 \mu \mathrm{M}$; ED, endothelial dysfunction induced by mechanical air perfusion; L-NAME, $\mathrm{N}^{\omega}$-nitro-L-arginine methyl ester hydrochloride, $100 \mu \mathrm{M}$; A967079, TRPA1 antagonist, $1 \mu \mathrm{M}$; ODQ, 1h[1,2,4]oxadiazolo[4,3-a]quinoxalin-1-one, $3 \mu \mathrm{M}$; Ezatiostat $1 \mu \mathrm{M}$; Nicotine $1 \mu \mathrm{M}$; --, experimental group not performed; (f), female. All SMA were from male mice except where indicated female, (f). $\mathrm{N}=3-6$ per group. ${ }^{*}, \mathrm{P}<0.05$ vs matched Control (PE+vehicle); \#, $0.05<P<0.10$ vs matched Control (PE+vehicle). 
Table 15. The sensitivity (effective concentration inducing 50\% relaxation; $\mathrm{EC}_{50}$; in $\mu \mathrm{M})$ of crotonaldehyde $(\mathrm{CR})$-induced vasorelaxation in PE pre-contracted murine superior mesenteric artery (SMA) in the absence and presence of functional endothelium or selective inhibitors.

\begin{tabular}{|c|c|c|}
\hline $\begin{array}{c}\text { SMA } \\
\text { (+treatment, N) }\end{array}$ & $\begin{array}{c}\mathrm{EC}_{50} \\
\text { (Control, PE+vehicle) }\end{array}$ & $\begin{array}{c}\mathrm{EC}_{50} \\
\text { (ED or } \mathrm{PE}+\text { inhibitor) }\end{array}$ \\
\hline $\mathrm{WT}+\mathrm{ED}$ & $5.7 \pm 1.0$ & $68.2 \pm 13.9^{*}$ \\
\hline WT+L-NAME & $6.0 \pm 2.0$ & $43.6 \pm 17.9^{*}$ \\
\hline $\mathrm{WT}+\mathrm{ODQ}$ & $3.9 \pm 1.6$ & $35.3 \pm 1.6^{*}$ \\
\hline WT+A967079 & $7.2 \pm 2.3$ & $12.1 \pm 2.1^{*}$ \\
\hline TRPA1-null & $17.9 \pm 7.4$ & $14.1 \pm 2.7$ \\
\hline WT+Ezatiostat & $4.3 \pm 0.7$ & $4.2 \pm 0.6$ \\
\hline GSTP-null & $5.9 \pm 0.8$ & -- \\
\hline WT+Nicotine & $3.3 \pm 0.8$ & $5.7 \pm 1.2$ \\
\hline WT (f) & $6.6 \pm 0.3^{*}$ & -- \\
\hline
\end{tabular}

Values $=$ means \pm SE. Abbr.: PE, phenylephrine, $10 \mu \mathrm{M}$; ED, endothelial dysfunction induced by mechanical air perfusion; L-NAME, $\mathrm{N}^{\omega}$-nitro-L-arginine methyl ester hydrochloride, $100 \mu \mathrm{M}$; A967079, TRPA1 antagonist, $1 \mu \mathrm{M}$; ODQ, $1 \mathrm{~h}$ [1,2,4]oxadiazolo[4,3-a]quinoxalin-1-one, $3 \mu \mathrm{M}$; Ezatiostat $1 \mu \mathrm{M}$; Nicotine, $1 \mu \mathrm{M}$; --, experimental group not performed; (f), female. All SMA were from male mice except where indicated (f). $\mathrm{N}=3-6$ per group. ${ }^{*}, \mathrm{P}<0.05$ vs matched Control (PE+vehicle). 
Table 16. Characteristics of tension oscillations induced by a high concentration of crotonaldehyde $(\mathrm{CR} ; 300 \mu \mathrm{M})$ in PE-precontracted superior mesenteric artery (SMA).

\begin{tabular}{|c|c|c|}
\hline SMA (+treatment) & $\begin{array}{c}\text { Frequency } \\
\text { (oscillations/min) }\end{array}$ & Amplitude (mN) \\
\hline WT+CR & $2.0 \pm 0.4$ & $2.0 \pm 0.3$ \\
\hline WT+CR+ED & $0^{*}$ & -- \\
\hline WT+CR+L-NAME & $1.3 \pm 0.2$ & $0.9 \pm 0.1$ \\
\hline WT+CR+ODQ & $2.1 \pm 0.6$ & $1.2 \pm 0.5$ \\
\hline WT+CR+A967079 & $1.7 \pm 0.3$ & $1.9 \pm 0.5$ \\
\hline TRPA1-null+CR & $1.7 \pm 0.3$ & $2.0 \pm 0.2$ \\
\hline WT+CR+Ezatiostat & $1.9 \pm 0.1$ & $2.1 \pm 0.2$ \\
\hline GSTP-null+CR & $1.9 \pm 0.6$ & $3.4 \pm 0.5$ \\
\hline WT+Nicotine & $1.8 \pm 0.2$ & $1.6 \pm 0.3$ \\
\hline WT (f) +CR & $1.4 \pm 0.6$ & $2.9 \pm 0.6$ \\
\hline Values = means \pm SE. Abbr.: PE, phenylephrine, $10 \mu \mathrm{M} ;$ WT, wild type; ED,
\end{tabular}

endothelial dysfunction; L-NAME, $\mathrm{N}^{\omega}$-nitro-L-arginine methyl ester hydrochloride, $100 \mu \mathrm{M}$; ODQ, 1h-[1,2,4]oxadiazolo[4,3-a]quinoxalin-1-one, $3 \mu \mathrm{M}$; A967079, TRPA1 antagonist, $1 \mu \mathrm{M}$; Ezatiostat $1 \mu \mathrm{M}$; Nicotine, $1 \mu \mathrm{M}$; (f), female. All SMA were from male mice except where indicated (f). $\mathrm{N}=3-10$ per group. ${ }^{*}, \mathrm{P}<0.05$ vs $\mathrm{WT}+\mathrm{CR}$. 
Fig. 24. Mechanisms of crotonaldehyde-induced (CR) relaxation in superior mesenteric artery (SMA). A) Representative traces of CR-stimulated relaxation of $\mathrm{PE}$-induced contraction without and with endothelium impairment due to $5 \mathrm{~min}$ of air perfusion. B) Summary data of CR-induced relaxation in isolated SMA precontracted with PE in the presence and absence of functional endothelium. C) Representative traces of CR-stimulated relaxation of PE-induced contraction in the absence and presence of L-NAME $(100 \mu \mathrm{M})$. D) Summary data of CR-induced relaxation in isolated SMA pre-contracted with $\mathrm{PE}$ in the absence and presence of L-NAME. E) Representative traces of CR-stimulated relaxation of PE-induced contraction in the absence and presence of the guanylyl cyclase (GC) antagonist, ODQ $(3 \mu \mathrm{M})$, added to bath after PE-induced contraction plateaued and prior to cumulative addition of CR. F) Summary data of CR-induced relaxation in isolated SMA pre-contracted with PE in the absence and presence of ODQ. Values = means \pm SE of 3-4 preparations. ${ }^{*}, P<0.05$ vs control. 

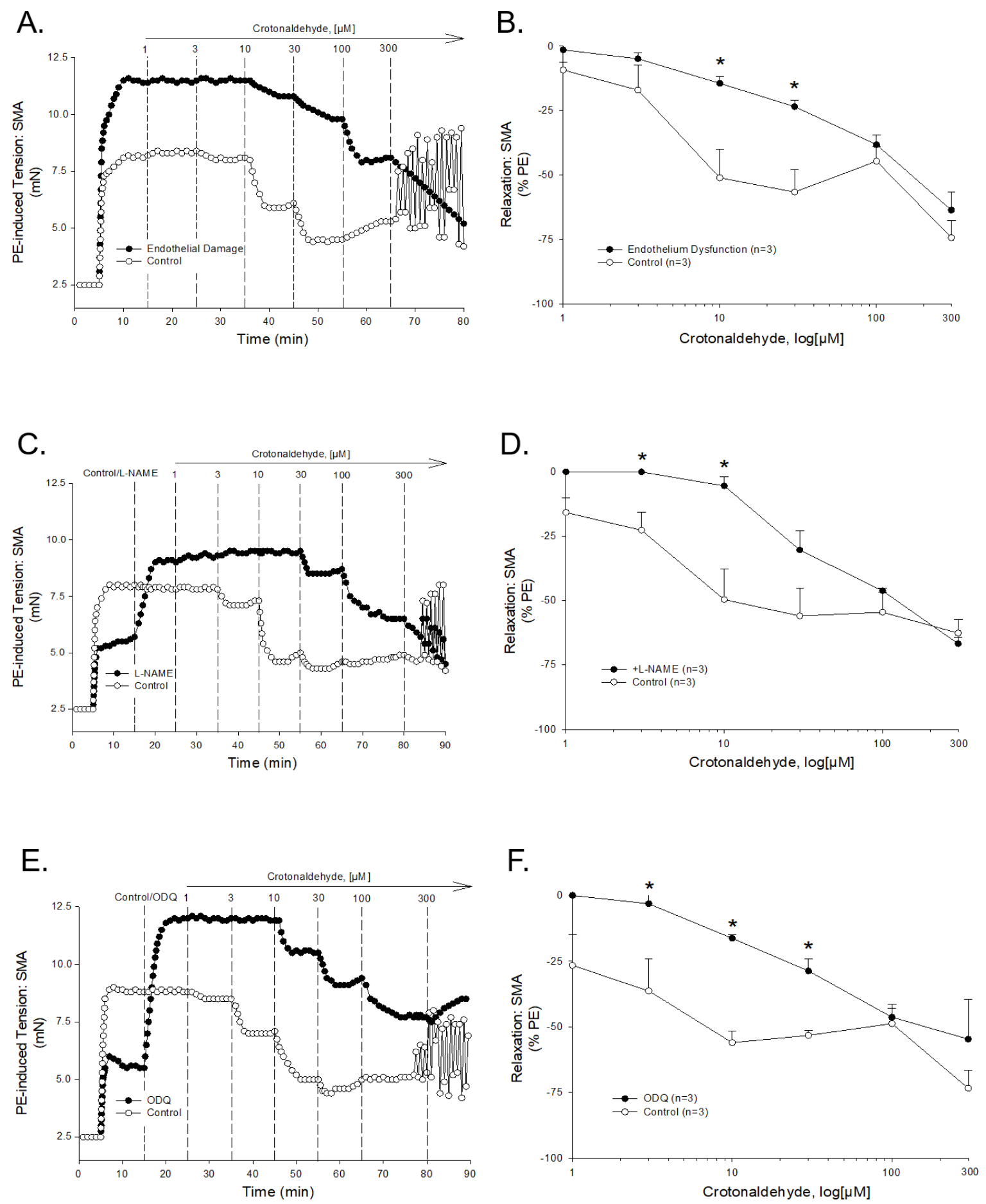
Fig. 25. Role of the TRPA1 channel in crotonaldehyde (CR)-induced relaxation in superior mesenteric artery (SMA). A) Representative traces of CR-stimulated relaxation of phenylephrine (PE) pre-contracted SMA in the absence (Control) and presence of the TRPA1 antagonist, A967079 $(1 \mu \mathrm{M})$ added after $\mathrm{PE}$-induced contraction plateaued and prior to cumulative addition of $\mathrm{CR}$ in wild type (WT) SMA. B) Summary data of CR-induced relaxation in isolated SMA pre-contracted with PE in the absence and presence of TRPA1 antagonist. C) Representative traces of CR-stimulated relaxation of PE pre-contracted SMA in the absence (Control) and presence of the TRPA1 antagonist, A967079 $(1 \mu \mathrm{M})$ added after $\mathrm{PE}$-induced contraction plateaued and prior to cumulative addition of CR in TRPA1-null SMA. D) Summary data of CR-induced relaxation in PE precontracted TRPA1-null SMA in the absence and presence of TRPA1 antagonist. E) Representative traces of CR-stimulated relaxation of PE pre-contracted SMA of WT and GSTP-null mice. F) Summary data of CR-induced relaxation in PE precontracted WT and GSTP-null SMA. G) Representative traces of CR-stimulated relaxation of phenylephrine (PE) pre-contracted SMA in the absence (Control) and presence of the GSTP antagonist, Ezatiostat $(1 \mu \mathrm{M})$ added after PE-induced contraction plateaued and prior to cumulative addition of CR in wild type (WT) SMA. H) Summary data of CR-induced relaxation in isolated SMA pre-contracted with $\mathrm{PE}$ in the absence and presence of GSTP antagonist. Values = means $\pm \mathrm{SE}$ of 3-4 preparations. ${ }^{*}, \mathrm{P}<0.05$ vs Control. 
A.

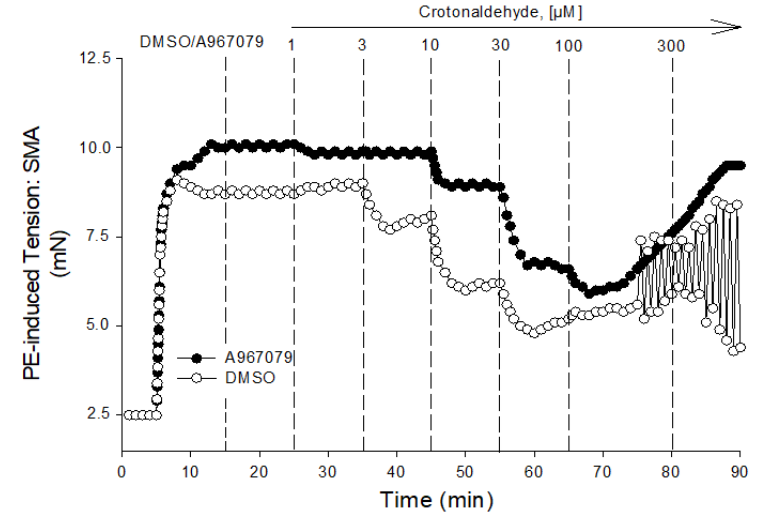

C.

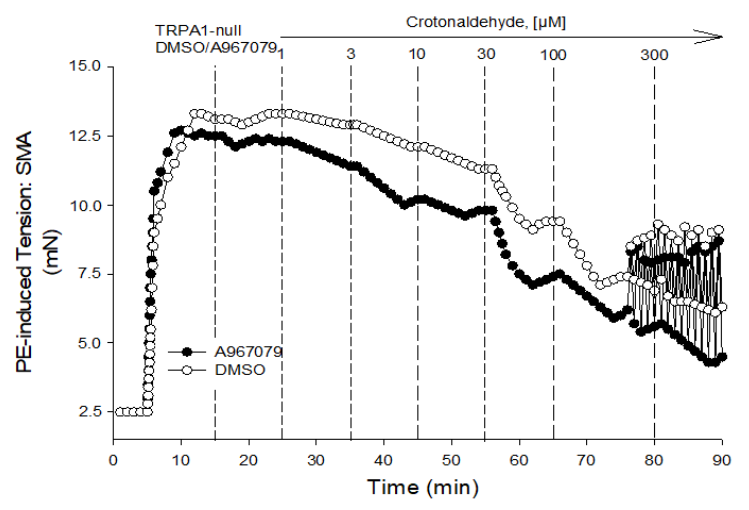

B.

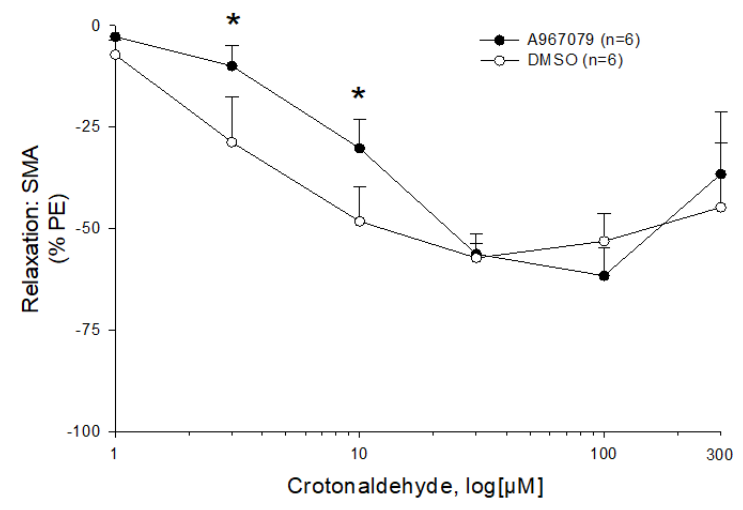

D.

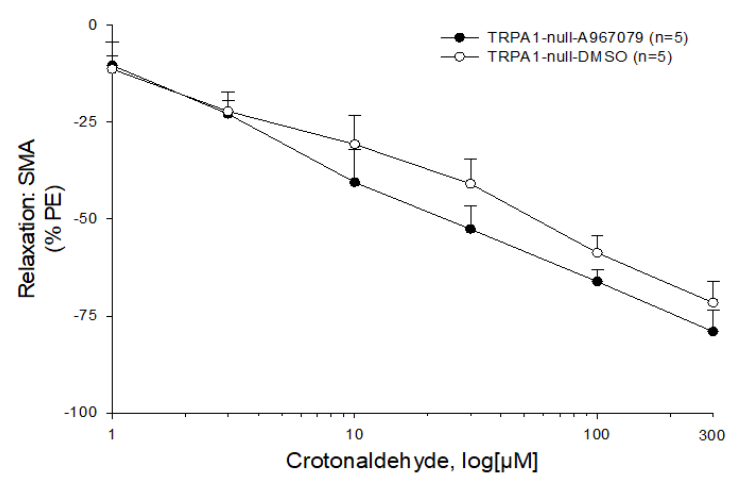




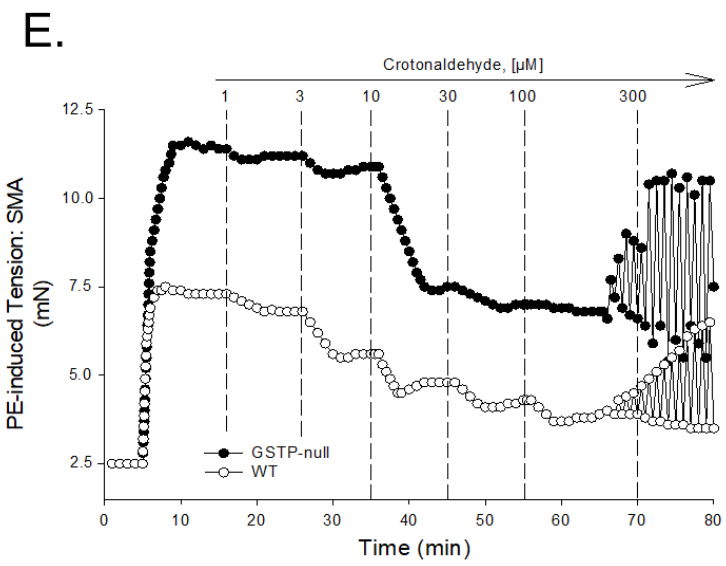

G.

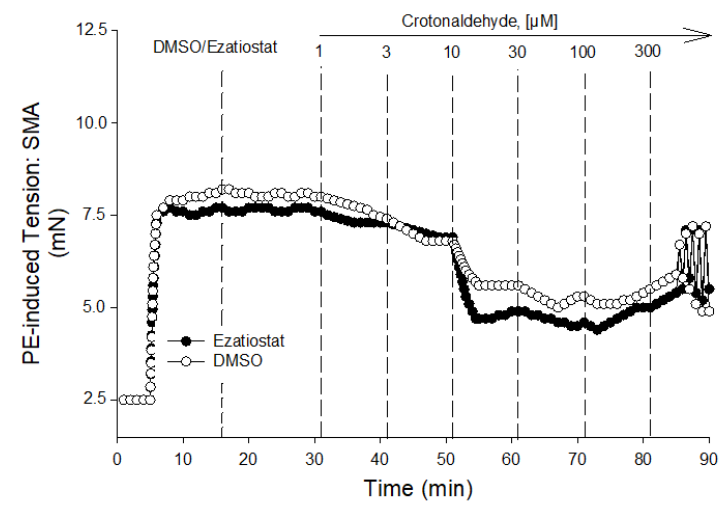

F.

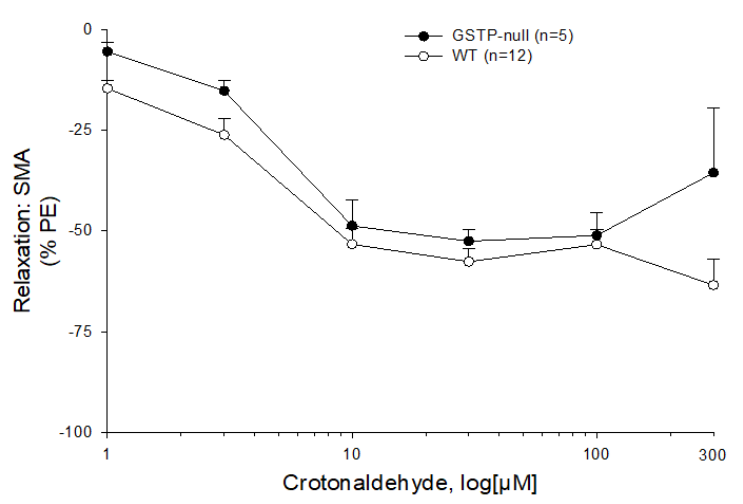

$\mathrm{H}$.

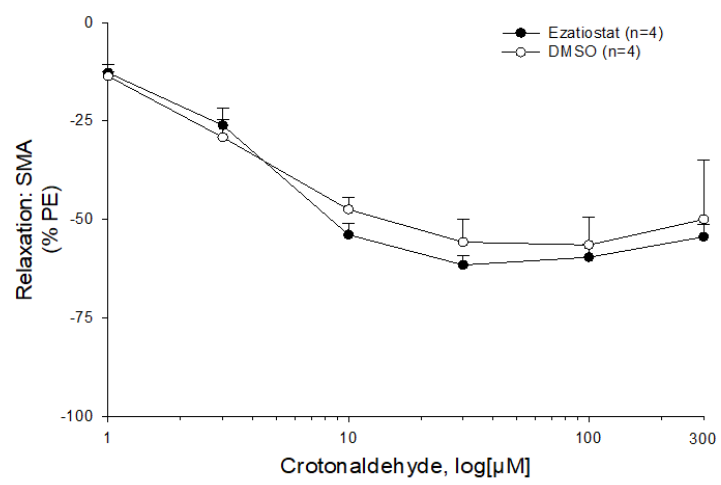


Fig. 26. Role of TRPA1 in AITC-induced relaxation of superior mesenteric artery (SMA), and localization in SMA and aorta. A) Representative traces of AITC-stimulated, concentration-dependent relaxation of PE-contracted SMA. B) Summary data of AITC-induced relaxation in PE-contracted SMA of WT and TRPA1-null mice in the absence (DMSO) and presence of TRPA1 antagonist, A967079 $(1 \mu \mathrm{M})$. Formalin-fixed, paraffin-embedded, cross-sections of murine SMA (C-E) and aorta (F-H) stained with H\&E (C, F). Immunofluorescent localization of TRPA1 alone in SMA (green, D) or with isolectin (red, endothelium) and DAPI (E). Immunofluorescent localization of TRPA1 alone in aorta (green, $\mathbf{G}$ ) or with isolectin (red) and a TRPA1 blocking peptide and DAPI $(\mathbf{H})$. All images were taken at 200x magnification (scale bar $=100 \mu \mathrm{m}$ ). Values $=$ means $\pm \mathrm{SE}$ of 3-4 preparations. ${ }^{*}, P<0.05$ vs Control. Abbr.: L, lumen. 
A.

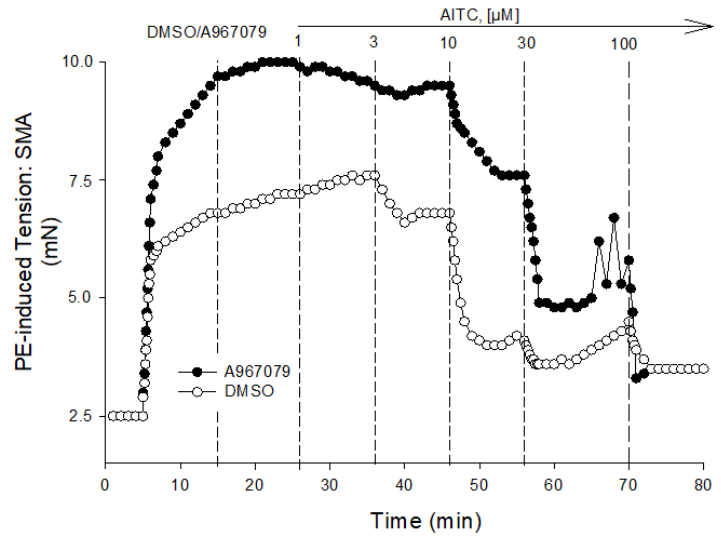

B.

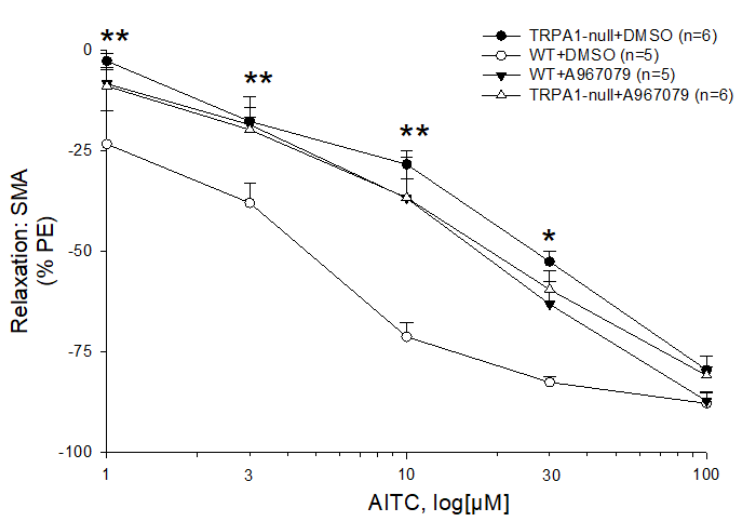

C.
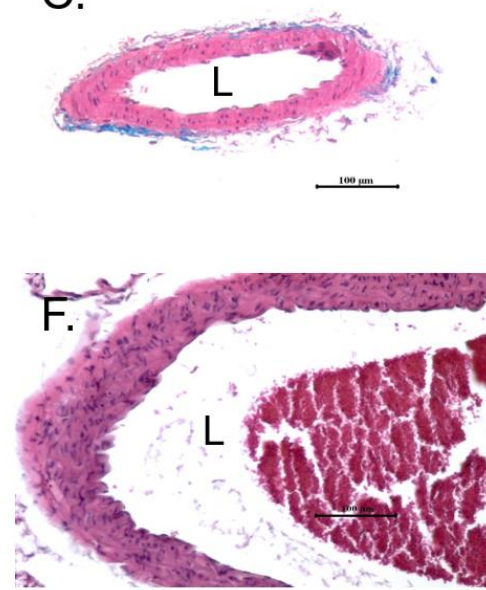

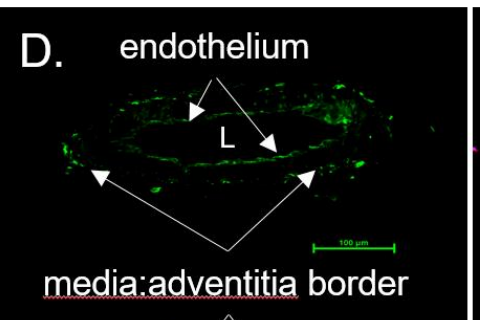

E.

endothelium

\section{G.}

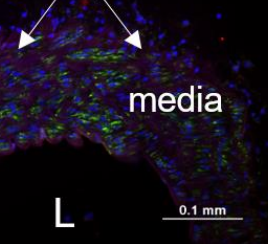

$\mathrm{H}$. media

L 
Fig. 27. Immunofluorescent localization of TRPA1 in dorsal root ganglion (DRG). Formalin-fixed, paraffin-embedded sections of murine DRG were stained with H\&E (A); DAPI only (blue) (B); TRPA1 antibody (red) and DAPI (C); or, TRPA1 antibody, TRPA1 blocking peptide, and DAPI (D). All images were imaged at 200x magnification (scale bar $=100 \mu \mathrm{m})$. 


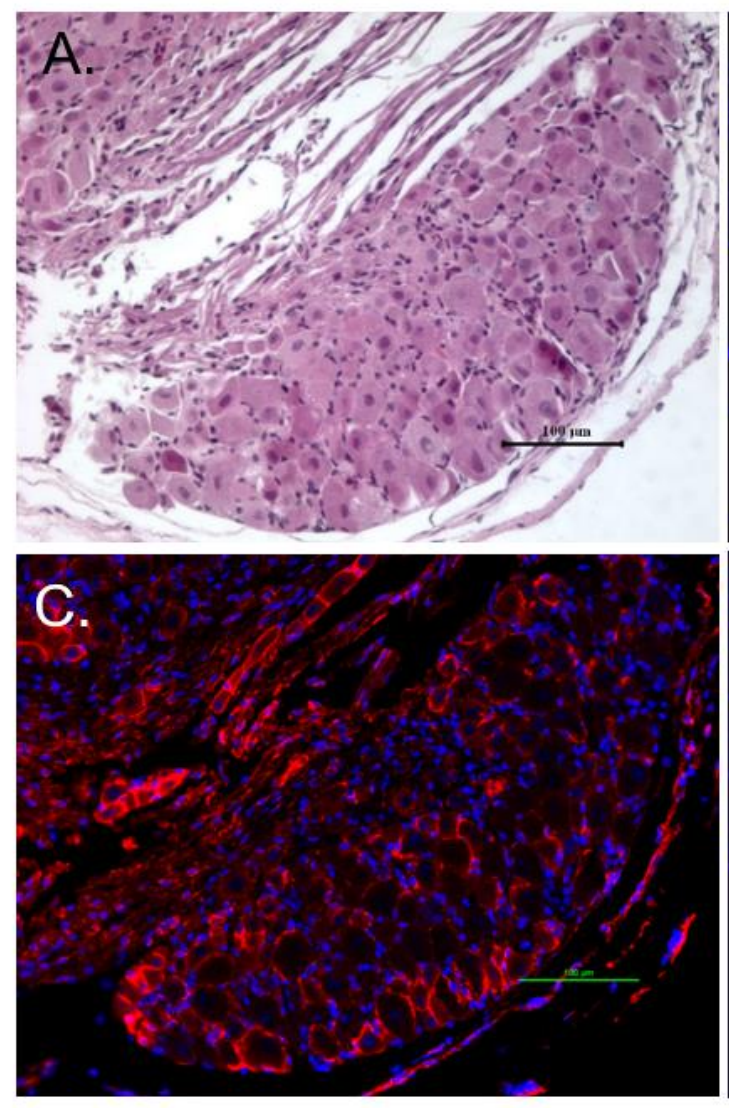

\section{B.}

D. 
Fig. 28. Toxicity of crotonaldehyde (CR) exposure in superior mesenteric artery (SMA). A-B) Ratio of $\mathrm{PE}_{2}$ (PE-induced tension post-CR [300 $\left.\mu \mathrm{M}\right]$ exposure) to $\mathrm{PE}_{1}$ ( $\mathrm{PE}$-induced tension pre-CR) (as \%) across treatment groups with untreated control group for comparison. B) Effects of $\mathrm{CR}$ and treatments on ACh-induced relaxation. Values $=$ means \pm SE of 3-6 preparations. ${ }^{*}, P<0.05$ vs untreated Control or vs CR control (B only). Abbr.: ED, endothelial dysfunction. 
A.

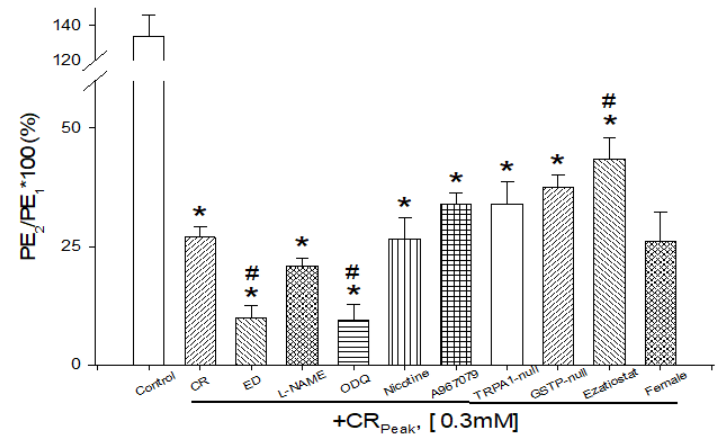

B.

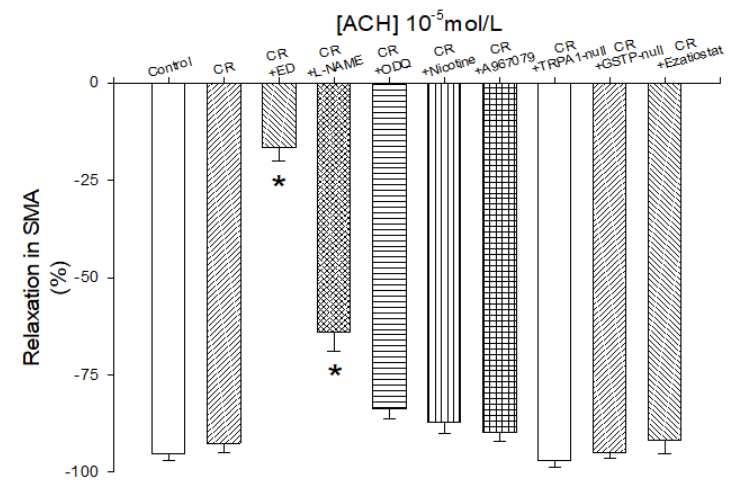


Table 17. Vasotoxicity of crotonaldehyde (CR) in superior mesenteric artery (SMA).

\begin{tabular}{|c|c|c|}
\hline SMA (+treatment) & PE $_{2} /$ PE $_{\mathbf{1}}(\%)$ & ACh (\%) \\
\hline WT (control) & $133.6 \pm 12.3$ & $-95.4 \pm 1.6$ \\
\hline WT+CR & $27.0 \pm 2.2^{*}$ & $-92.6 \pm 2.5$ \\
\hline WT+CR+ED & $10.0 \pm 2.5^{\star \#}$ & $-16.7 \pm 3.3^{*}$ \\
\hline WT+CR+L-NAME & $21.0 \pm 1.7^{*}$ & $-64.2 \pm 4.8^{*}$ \\
\hline WT+CR+ODQ & $9.5 \pm 3.2^{* \#}$ & $-83.9 \pm 2.4$ \\
\hline WT+CR+A967079 & $34.0 \pm 2.4^{*}$ & $-89.9 \pm 2.3$ \\
\hline TRPA1-null+CR & $34.0 \pm 4.7^{*}$ & $-97.1 \pm 1.8$ \\
\hline WT+CR+Ezatiostat & $43.5 \pm 4.5^{\star \#}$ & $-91.9 \pm 3.4$ \\
\hline GSTP-null+CR & $37.5 \pm 2.5^{*}$ & $-95.1 \pm 1.3$ \\
\hline WT+CR+Nicotine & $26.6 \pm 4.5^{*}$ & $-87.3 \pm 2.9$ \\
\hline WT (f) +CR & $26.1 \pm 6.2^{*}$ & $-93.9 \pm 3.3$ \\
\hline
\end{tabular}

Values = means \pm SE. Abbr.: PE, phenylephrine, $10 \mu \mathrm{M}$; ACh, acetylcholine chloride, WT, wild type; ED, endothelial dysfunction; L-NAME, $\mathrm{N}^{\omega}$-nitro-L-arginine methyl ester hydrochloride, $100 \mu \mathrm{M}$; ODQ, 1h-[1,2,4]oxadiazolo[4,3-a]quinoxalin1-one, $3 \mu \mathrm{M}$; A967079, TRPA1 antagonist, $1 \mu \mathrm{M}$; Ezatiostat $1 \mu \mathrm{M}$; Nicotine, $1 \mu \mathrm{M}$, $(f)$, female. The $P E_{2} / P E_{1}$ tension (\%) was calculated by dividing $P E-$ induced tension post-CR-exposure ( $\mathrm{PE}_{2}$ ) by $\mathrm{PE}$-induced tension pre-CR (i.e., $\mathrm{PE}_{1}$ ) and multiplied by 100 . ACh-induced relaxation was calculated as the \% reduction in $\mathrm{PE}_{2}$-induced tension. All SMA were from male mice except where indicated (f). $\mathrm{N}=3-10$ per group. ${ }^{*}, \mathrm{P}<0.05$ vs WT (male) (control). ${ }^{*}, \mathrm{P}<0.05$ vs WT+CR. 
Fig. 29. Effects of nicotine on CR-induced relaxation in SMA. A) Representative trace of concentration dependent $(0.1 \mu \mathrm{M}-1 \mathrm{mM})$ nicotineinduced relaxation of PE pre-contracted SMA. B) Nicotine at $1 \mu \mathrm{M}$ had no effect on tension of PE-precontracted SMA yet significantly decreased efficacy (10, 30 and $100 \mu \mathrm{M}$ ) of subsequent $\mathrm{CR}$-induced vasorelaxation (Table 1). Values $=$ means \pm SE of 6 preparations. ${ }^{*}, P<0.05$ vs matched Control; ${ }^{\#}, 0.05<\mathrm{P}<0.10$ vs matched Control. 
A.

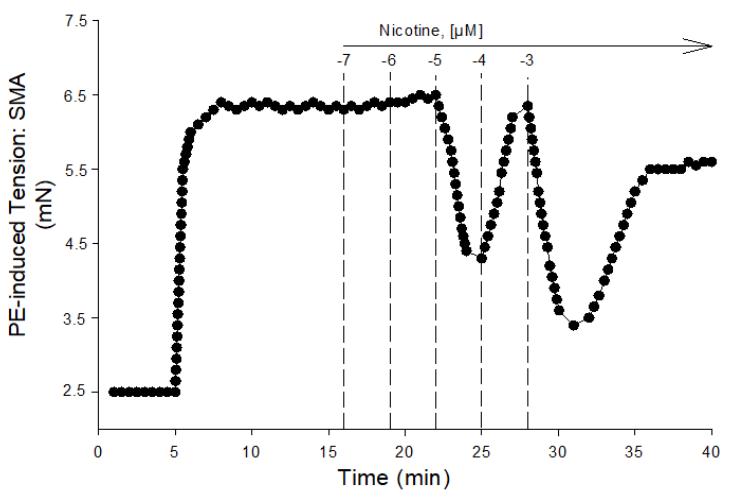

B.

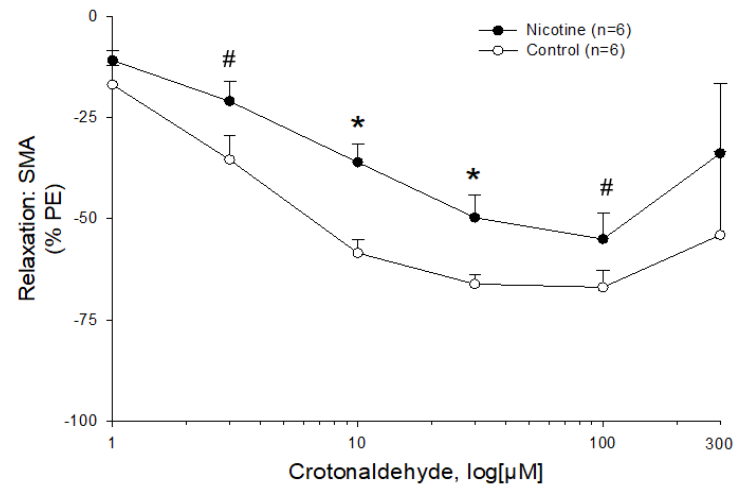




\section{Discussion}

To our knowledge, this is the first study to show the vasoreactivity of CR and its mechanisms of action that include TRPA1. The mechanisms of CR-induced vasorelaxation are similar to formaldehyde, including dependence on TRPA1, endothelium, NO formation and guanylyl cyclase activation (Jin, Jagatheesan, et al., 2019). The robust and sensitive relaxation is reminiscent of actions described for acrolein at low concentrations in perfused rat mesenteric bed (Awe et al., 2006), isolated rat coronary artery (D. J. Conklin et al., 2001a), and isolated mouse aorta (D. J. Conklin, Haberzettl, et al., 2009a). Moreover, we show that, also like acrolein (D. J. Conklin et al., 2001a), CR at higher concentrations induces tension oscillations ("spasms") and suppresses contractility (toxicity) in SMA. This continuum from potent relaxation to vascular toxicant belies the importance of studying these responses as CR is abundant in foods, beverages, occupational settings, and especially in combustion-derived smoke, i.e., tobacco smoke, wildfires, etc... Thus, appreciating the avidity of unsaturated aldehydes for altering vascular wall function is a first step toward understanding how exposure to CR may increase cardiovascular disease risk.

It is unsurprising that vascular TRPA1 is involved in CR-induced vasorelaxation. Activation of TRPA1 can lead to release of vasoactive peptides, substance P (SubP) and CGRP (Trevisan et al., 2016) that can induce vasodilation and inflammatory responses (Achanta, Chintagari, Brackmann, Balakrishna, \& Jordt, 2018; D. J. Conklin, 2016). The mechanism of CR-induced TRPA1-mediated relaxations in SMA, however, appears to be dependent largely on endothelium- 
localized TRPA1 as we show that endothelial impairment either by air perfusion or L-NAME shifts the sensitivity of the CR response rightward to a degree similar to that of either TRPA1 antagonist treatment or by use of TRPA1-null SMA. This is mirrored by relaxation of known TRPA1 agonist AITC that is equally shifted by both A967079 and TRPA1 deletion. Nevertheless, we cannot fully rule out that other cells in the isolated SMA, e.g., perivascular nerves, contribute to CR's mechanism of relaxation. Moreover, there are at least 2 mechanisms mediating the full relaxation of CR (see Fig. 30): 1) endothelium; and, 2) time-dependent, irreversible change in VSMC contractile machinery, e.g., closure of VSMC calcium channels. Although the latter conclusion has limited experimental support, we infer it because PE contraction is significantly impaired both pre- and post-CR exposure and washouts; endothelium is intact because ACh-induced relaxation is unaffected post-CR exposure (no endothelium dysfunction); and, previous experience with similar vascular toxicity of acrolein led to findings consistent with a role of external calcium (D. J. Conklin et al., 2006).

Cigarette smoke is a major source of exogenous CR (Goniewicz et al., 2017; Lorkiewicz et al., 2019). We show that exposure of mice to MCS increases excretion of the CR metabolite, HPMMA, by $2.5 x$, indicating there is a fair amount of CR produced endogenously in mice. Our estimation of circulating blood levels of CR puts it in the low end of the vasoactive range - a novel consideration. Given that acrolein and CR are highly reactive unsaturated aldehydes, it is hard to fathom these molecules floating as free aldehydes in the blood, and we do not think that. However, the metabolites of CR and acrolein certainly are carried in the blood, and 
we hypothesize that these metabolites may be vasoactive, and this possibility should be investigated further. Nonetheless, many unsaturated aldehydes are noted potent vasodilators including acrolein (Awe et al., 2006), 4-hydroxynonenal (4HNE) (Martinez et al., 1994; Romero et al., 1997) and cinnamaldehyde (Pozsgai et al., 2010; Yanaga et al., 2006), and now CR -- all these are known ligands of TRPA1. These potent dilatory effects may contribute to a variety of physiological processes such as postprandial hyperemia or flow-mediated dilation (FMD) or response to injury/infection, but too much of any of these aldehydes may lead to pathological changes including vasospasm, atherosclerosis and vascular toxicity (Boor \& Conklin, 2008; D. J. Conklin et al., 2006; D. J. Conklin et al., 2001a). Although these parent aldehydes can covalently modify intracellular aminoterminus free cysteines to activate TRPA1 (Macpherson et al., 2007), it is unclear if their metabolites can do this as well or if there are other mechanisms of activating TRPA1 channels.

Nicotine is also an important component of tobacco smoke, and nicotine activates the sympathetic nervous system that can increase cardiovascular disease risk factors such as heart rate and blood pressure and decrease heart rate variability (HRV). These changes, in turn, affect myocardial remodeling, impair lipid metabolism, and stimulate inflammation (Benowitz \& Burbank, 2016; Dutta et al., 2012). The plateau concentration of nicotine in the blood of smokers throughout the day averages $40 \mu \mathrm{g} / \mathrm{L}(0.25 \mu \mathrm{M})$ (Moyer et al., 2002), and thus, we tested whether effects of super-pharmacological level of nicotine $(1 \mu \mathrm{M})$ could alter CR effects in PE pre-contracted SMA. Although nicotine induces relaxation at high 
concentrations ( $\geq 10 \mu \mathrm{M})$, it is inactive at $1 \mu \mathrm{M}$ (see Fig. 29A); yet, it modestly suppresses CR-induced relaxation. The mechanism of this effect is unclear, but inhaled nicotine at a high concentration $(1 \mathrm{mM})$ is thought to activate sensory TRPA1 channel and stimulate airway constriction reflex (Talavera et al., 2009). Our previous studies show that nicotine $(1 \mu \mathrm{M})$ has no effect on formaldehyde- or acetaldehyde-induced relaxations in PE-contracted SMA (Jin, Jagatheesan, et al., 2019; Jin, Lorkiewicz, et al., 2019), indicating this effect appears specific for CR. Inhibition of CR vasorelaxation may promote the sympathomimetic effects of nicotine alone, e.g., increased blood pressure, perhaps counteracting vasodepressor effects of $C R$.

In conclusion, this study describes a sensitive mechanism of CR-induced vasorelaxation that is sequentially dependent on endothelium-localized TRPA1 channel, NO, and a VSMC GC pathway in SMA (but not in aorta), which may play an important role in regulating blood flow/blood pressure at low endogenous levels of CR. Smooth muscle impairment at high concentrations (300 $\mu \mathrm{M})$ of CR evokes tension oscillations reminiscent of "spasms" observed with acrolein in vitro another vasotoxic and TRPA1 agonist compound. Future studies of the in vivo contribution of CR and TRPA1 to cardiovascular physiology and pathophysiology are required to better understand how exposure to these reactive aldehydes confer increased cardiovascular disease risk. 
Fig. 30. Cartoon depicting the mechanisms of crotonaldehyde (CR) induced vasorelaxation in SMA. CR induces a sensitive and sequential mechanism dependent on activation of Transient Receptor Potential Ankyrin 1 (TRPA1) cation channels, endothelial cell-based eNOS, NO-mediated GC activation leading to VSMC hyperpolarization and relaxation. These steps were significantly impaired by a selective antagonist or treatment, including endothelium impairment (by air perfusion), eNOS inhibition (L-NAME), guanylyl cyclase inhibitor (GC; ODQ), and TRPA1 inhibitor (A967079). An additional, but less sensitive pathway of CRmediated relaxation likely involves closure of VSMC calcium channels. Potential role of VSMC-localized TRPA1 channel activation (dashed line) in CR-induced relaxation, tension oscillations or vascular toxicity is uncertain. Glutathione $S$ transferase P (GSTP) deletion had no effect on CR actions in SMA. 


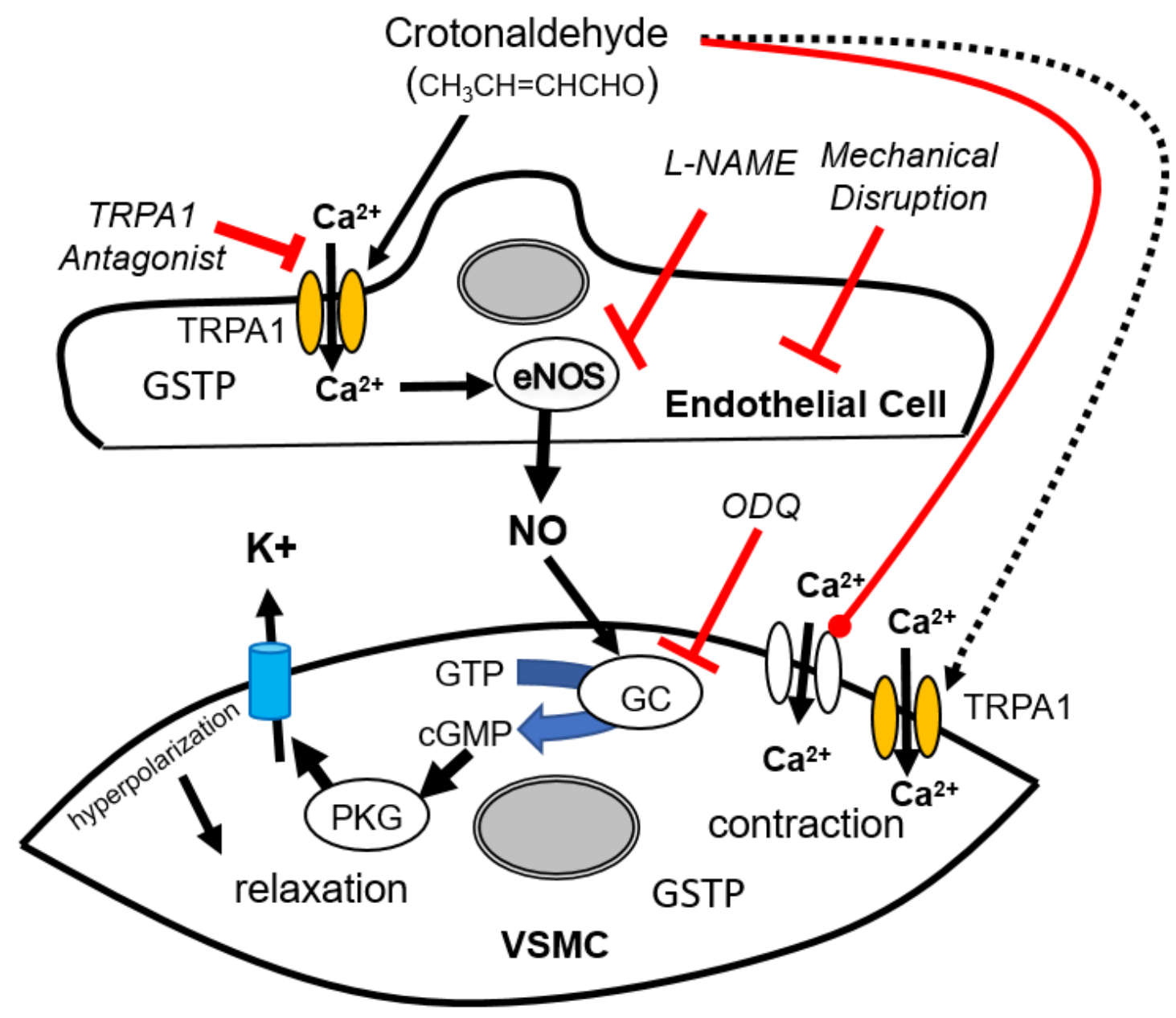




\title{
CHAPTER VI
}

\author{
VASCULAR EFFECTS OF INHALED ALDEHYDES IN VIVO: \\ ROLE OF ENDOTHELIUM- AND TRANSIENT RECEPTOR POTENYTIAL \\ ANKYRINE-1 (TRPA1) - DEPENDENT MECHANISM
}

Introduction Formaldehyde (FA) and acetaldehyde (AA) are small, saturated aldehydes, and crotonaldehyde (CR) is a highly electrophilic $\alpha, \beta$-unsaturated aldehyde, they are all abundant in the environment and in the aerosols of tobacco as well as in many foods and beverages. Because of high-level exposures in users of tobacco products and are well-known associated with the development of cardiovascular disease (CVD), including atherosclerosis. We explored the effects of FA, AA and CR exposures in isolated murine blood vessels. Objective The purpose of this study was to explore the acute and chronic cardiovascular toxicity of inhaled aldehydes in mice in vivo; and explore the possible mechanisms. Method and Results C57BL/6J (wild type, WT) mice were chronically exposed to CR or filtered room air for 12 weeks ( $1 \mathrm{ppm}, 5$ $\mathrm{d} /$ week). Blood pressure was significantly lower in the CR group than in the air control group, while heart rate was unchanged. Hemodynamic changes 
were accompanied by changes in aortic vasoreactivity ex vivo. Following $12-w k$ CR chronic exposure, there was no change in aortic response to phenylephrine (PE), however, the sensitivity of acetylcholine- (ACh) and sodium nitroprussideinduced relaxation were significantly altered in the CR-exposed mice. To dissect the mechanism of CR-induced vascular changes, we performed acute 4-day CR exposures ( 1 and 3 ppm). Acute CR exposures also altered aortic responses to ACh and SNP. Based on these findings, we tested for the role of the transient receptor potential ankyrin 1 (TRPA1) channel. In CR-exposed TRPA1-null mice (4-days, 1ppm), there were no changes in aortic responses to any agonist (PE, ACh, and SNP) compared with air group, indicating a distinct role of TRPA1. Interestingly, there also were no differences in aortic responses in acutelyexposed female C57BL/6J mice (1 ppm, 6h/day, 4days), indicating sexdependence. WT mice were exposed to FA or AA gas (5ppm, 6h/day) for 4days, FA from $30-1,500 \mathrm{Mm}$ or AA from 1-100 mM strongly and reversibly inhibited agonist-induced contractions. There was no difference in the sensitivity and relaxation of acetylcholine (ACh) and sodium nitroprusside (SNP). So, short-term inhalation exposure to FA or AA at levels present in tobacco product aerosols did not alter the direct effects of these aldehydes. Conclusions These consistent vascular effects indicate that $\mathrm{CR}$ is vasoactive at low concentrations and chronic exposure to CR may lead to compensatory changes in the vasculature to offset excessive smooth muscle relaxation and hypotension, which may lead to vascular disease. Future studies are required to better understand the mechanisms by CR exposure modifies cardiovascular pathophysiology, and the 
specific role of TRPA1 and sex in CVD such as atherosclerosis. Those two saturated aldehydes, FA and AA, have limited direct toxicity in large arteries prior inhalation exposure indicating these agents may not be involved in tobaccorelated endothelium dysfunction.

\section{Introduction}

Smoking is the most significant preventable factor in the development of cardiovascular disease (CVD) (Association., 2014; Bhatnagar, 2006), has been shown to increase the risk of myocardial infarction, coronary artery disease, atherosclerosis and stroke (Ambrose \& Barua, 2004). Tobacco use is responsible for an estimated 7 million global deaths each year (Organization., 2017), most deaths due to CVD (Haussmann, 2012). Extensive evidence demonstrates that exposure to both mainstream cigarette smoke (MCS) (Administration., 2012; U.S. Department of Health and Human Services, 2014) or second-hand tobacco smoke (Prevention., 2017; U.S. Department of Health and Human Services, 2014) releases high concentration of aldehydes and increases the risk for CVD.

More than $90 \%$ of the non-cancer health risk associated with smoking (i.e., cardiovascular and pulmonary disease risk) is attributable to aldehydes (Haussmann, 2012). The unsaturated aldehydes (Administration., 2012; Smith \& Fischer, 2001; van Andel I, 2017), including acrolein and crotonaldehyde (CR), some studies showed that MCS contains acrolein (50-70 ppm) and 
crotonaldehyde (CR) (1-53 $\mu \mathrm{g} /$ cigarette), these units are very different (Alwis, deCastro, Morrow, \& Blount, 2015; Pazo et al., 2016), though they are classified as the most significant toxins in tobacco smoke (Haussmann, 2012; Stabbert et al., 2017). Our previous study showed that e-cigarette contains very high concentrations of FA and AA (Ogunwale et al., 2017). Aldehyde also generated by burning coal and gasoline, FA and AA approximately $71.4 \%$ of the gasoline exhaust from cars (Committee on, 1981). As we know, it's very difficult to measure aldehyde directly since it's highly reactive. So, even if it is formed in relatively small amounts, are likely to be of greater toxicological because of their high toxicity.

Because CR is physically and chemically similar to acrolein, we expected that the cardiovascular toxicity of $\mathrm{CR}$ in vivo may be similar to acrolein exposure, but surprisingly there are limited data on CR toxicity in vivo. CR has been shown to induce inflammation, oxidative stress and apoptosis in epithelial cells (Liu et al., 2010a, 2010b) and to cause cardiomyocyte dysfunction (Pei et al., 2014), but CR inhalation studies have not been performed. Both acute and chronic inhalation exposure to acrolein has been shown and induce endothelium dysfunction in mice (D. J. Conklin, Haberzettl, Lesgards, et al., 2009). Acrolein ingestion promotes pro-atherosclerotic changes and atherosclerosis (D. J. Conklin et al., 2010; Srivastava et al., 2011). Similarly, inhalation exposure to 1,3-butadiene, an upstream precursor of $\mathrm{CR}$, accelerates arteriosclerotic plaque development in cockerels (Penn \& Snyder, 1996b). Another epidemiological study expressed nearly $50 \%$ increase in the standardized mortality ratio (SMR) 
for atherosclerotic heart disease that was associated with environmental butadiene exposure (Matanoski et al., 1990). However, whether CR could promote the atherosclerotic effects of 1,3-butadiene exposure has not been studied.

The direct vascular toxicity of $C R$ inhalation remains unexplored, not to mention about the mechanisms by which inhaled CR may induce cardiovascular effects. From our previous studies, we show that the transient receptor potential ankyrin 1 (TRPA1) receptor is a pulmonary irritant receptor, mediate responses to inhaled compounds such as tobacco smoke and acrolein and blocked the relaxation of FA in vitro (D. J. Conklin, 2016; D. J Conklin et al., 2017; Jin, Jagatheesan, et al., 2019). Furthermore, the negative cardiovascular effects of inhaled acrolein (at levels in cigarette smoke) are TRPA1-dependent (Kurhanewicz, Ledbetter, Farraj, \& Hazari, 2018; Kurhanewicz et al., 2017). Yet the role of TRPA1 in potential cardiovascular toxicity of inhaled CR is unknown. To address these gaps in knowledge regarding potential cardiovascular toxicity (and its mechanisms) of $\mathrm{CR}$, healthy adult male and female C57BL/6 mice were exposed acutely or chronically (12 weeks; $1 \mathrm{ppm}$ ) by inhalation to $\mathrm{CR}$, and biomarkers of cardiovascular system harm were measured. To probe the role of TRPA1, we performed select CR exposures in TRPA1-null mice (D. J. Conklin et al., 2017). 


\section{Material and Methods}

Chemicals and Solutions.

Reagent grade chemicals were purchased from Sigma-Aldrich (St. Louis, MO) or other commercial sources as indicated: A967079 (AdooQ); acetylcholine chloride (ACh); 4-aminopyridine (4-AP); crotonaldehyde (CR); allyl isothiocyanate (AITC); 1h-[1,2,4]oxadiazolo[4,3-a]quinoxalin-1-one (ODQ); $\mathrm{N}^{\omega}{ }^{\omega}$-nitro-L-arginine methyl ester hydrochloride (L-NAME); L-phenylephrine hydrochloride (PE); sodium nitroprusside (SNP).

Krebs physiological salt solution (PSS) for aorta was (in $\mathrm{mM}$ ): $\mathrm{NaCl}, 119$; $\mathrm{KCl}, 4.7 ; \mathrm{CaCl}_{2}, 1.6 ; \mathrm{MgSO}_{4} \bullet 7 \mathrm{H}_{2} \mathrm{O}, 1.2 ; \mathrm{KH}_{2} \mathrm{PO}_{4}, 1.2 ; \mathrm{NaHCO}_{3}$, 25; glucose, 5.5; $\mathrm{pH}$ 7.4. High $\mathrm{K}^{+} \mathrm{PSS}(60 \mathrm{mM})$ substituted equimolar $\mathrm{K}^{+}$for $\mathrm{Na}^{+}$.

Mice and Crotonaldehyde Exposure

Mice.

Male and female C57BL/6 mice were obtained from The Jackson Laboratories (Bar Harbor, ME). Male TRPA1-null mice (on a C57BL/6 background) were from a breeding colony at the Univ. Louisville (D. J Conklin et al., 2017). All mice were treated according to the Guiding Principles for the Care and Use of Animals in Research and Teaching as adopted by the American Physiological Society, and all protocols were approved by the Univ. Louisville Institutional Animal Care and Use Committee. Before and during exposures, mice were housed under pathogen-free conditions, controlled temperatures, and a 12h:12h light:dark cycle. 
Mice were maintained on a standard chow diet (Rodent Diet 5010, 4.5\% fat by weight, LabDiet; St. Louis, MO).

Aldehydes exposures.

To parallel our previous study of chronic acrolein toxicity in mice at $1 \mathrm{ppm}$ exposure in mice (Daniel J. Conklin et al., 2017), we used 1 ppm CR. Mice were exposed to either HEPA- and charcoal-filtered air or CR (1 or 3 ppm, 6h/day) for 4 days or 12 weeks ( $1 \mathrm{ppm}$; 6h/day, 5 days/week) using a custom exposure system and certified permeation tubes (Kin-Tek; LaMarque, TX) as previously described (Daniel J. Conklin et al., 2017). Immediately following the final exposure, mice were anesthetized with sodium pentobarbital $(\approx 150 \mathrm{mg} / \mathrm{kg}$, i.p.) followed by ventral thoracotomy and exsanguination via cardiac puncture for blood collection in EDTAcoated syringes. Organs were removed, weighed, and snap frozen in $\mathrm{N}_{2}$ and stored at $-80^{\circ} \mathrm{C}$ until further analysis. Mice were exposed to either HEPA- and charcoal-filtered air, FA or AA (1 or $5 \mathrm{ppm}, 6 \mathrm{~h} /$ day) for 4 days using the same exposure system and methods.

Non-invasive blood pressure.

Blood pressure was measured in male mice using the CODA tail-cuff blood pressure system (Kent Scientific; Torrington, CT) as described (Daniel J. Conklin et al., 2019). Mice were acclimated for 5 consecutive days to the CODA system prior to collecting data. During the chronic exposure study, blood pressure measurements were made immediately following an exposure period once per week beginning in the second week of exposure and continuing for the remainder 
of the study. Briefly, mice ( $n=15$ in both control and exposure groups) were put in a holding chamber on a warming platform, and 25 measurement cycles were performed on each mouse with a five second interval between each cycle (approx. $30 \mathrm{~min})$.

Hemodynamics by telemetry.

To measure blood pressure and heart rate real-time in CR exposure, mice were implanted with pressure cannula in aortic arch for radiotelemetry (PA-C10; DSI, St. Paul, MN). Naïve mouse was given 1-week post-surgery to recover before being placed in an exposure chamber for $15 \mathrm{~min}$ of baseline recording prior to onset of CR exposure (9 min; 1 ppm; $2 x$ over $1 \mathrm{~h}$ ), and systolic blood pressure $(\mathrm{mmHg})$, diastolic blood pressure $(\mathrm{mmHg})$, mean blood pressure $(\mathrm{mmHg})$, pulse pressure $(\mathrm{mmHg})$, and heart rate (bpm) were continuously recorded $(1 \mathrm{kHz})$.

\section{Echocardiography.}

Echocardiography was performed in chronically-exposed male C57BL/6 mice using a Vevo-770 echocardiography system (FUJIFILM Visual Sonics; Toronto, Canada). Body temperature was maintained $\left(36 \cdot 5-37.5^{\circ} \mathrm{C}\right)$ using a rectal thermometer interfaced with a servo-controlled heat lamp. Mice were anesthetized with $2 \%$ isoflurane and maintained under anesthesia with $1.5 \%$ isoflurane during the procedure. The 707-B (30MHz) scan head was used to obtain 2D images (100 fps) of the parasternal long axis. M-modes were taken from the same anatomical position. An apical four-chamber view of the heart was obtained, and pulse wave Doppler readings were taken of the mitral valve. Pulse wave Doppler readings 
were also taken in the ascending aorta, the innominate artery, and the left common carotid artery and in the descending aorta distal the left subclavian artery. Additional pulse wave Doppler readings were taken at the branches of the descending aorta and both the right and left renal arteries. Pulse wave velocity was calculated by dividing the distance $(\mathrm{mm})$ between the left subclavian artery and the right and left renal arteries by the pulse wave travel time (ms).

\section{Vascular Reactivity}

Aorta isolation and preparation. Exposed mice were anesthetized (sodium pentobarbital, $\approx 150 \mathrm{mg} / \mathrm{kg}$, i.p.) and the aorta was removed via mid-ventral thoracotomy. Aortas were cleaned of perivascular adipose tissue and cut into rings. Thoracic aorta rings (3-4 $\mathrm{mm})$ were hung on stainless steel hooks in $15-\mathrm{ml}$ waterjacketed organ baths or in 5-ml heated organ baths of a MultiWire Myograph System 620M (DMT; Hinnerup, Denmark). The organ baths contained PSS bubbled with $95 \% \mathrm{O}_{2}: 5 \% \mathrm{CO}_{2}$ at $37^{\circ} \mathrm{C}$. After 10 min without tension, aorta rings were equilibrated to $\approx 1 \mathrm{~g}$ loading tension over $1 \mathrm{~h}$. All rings were stimulated with High $\mathrm{K}^{+}$to test for viability, washed three times with PSS over $30 \mathrm{~min}$, reequilibrated to $1 \mathrm{~g}$ (aorta) of resting tension. The rings were then stimulated again with High $\mathrm{K}^{+}$followed by three bath changes and re-equilibration to resting tension. Transducer signals were recorded using LabChart software.

Post-aldehydes exposure evaluation. To test whether CR exposure altered vascular reactivity, the following responses were measured: 1) contractions induced by High $\mathrm{K}^{+}$; 2) concentration-dependent contractions of phenylephrine 
$(\mathrm{PE})$; 3) concentration-dependent relaxations of acetylcholine (ACh) in PEprecontracted rings; and, 4) concentration-dependent relaxations of sodium nitroprusside (SNP; nitric oxide donor) in PE-precontracted rings. Measures of efficacy (Emax: contractions normalized to aortic length; percentage relaxation of PE contraction)(Jin, Lipinski, \& Conklin, 2018) and sensitivity $\left(E_{50}\right.$, effective concentration producing $50 \%$ response, i.e., cumulative concentration responses normalized to $100 \%$ with interpolation of $\mathrm{EC}_{50}$ ) were calculated (Conklin et al., 2009).

Histology and TRPA1 Immunofluorescence.

Thin sections $(4 \mu \mathrm{m})$ of formalin-fixed, paraffin-embedded SMA, aorta and dorsal root ganglia (DRG; positive control) of WT mice were stained with H\&E or TRPA1 antibody (1:200; Alomone Labs, Israel; Cat. \#: ACC-037). To label endothelial cells, blood vessel sections were co-stained with isolectin GS-IB4 Alexa Fluor 594 conjugate (1:200 dilution; Invitrogen; Cat. \#: I21413). Images of H\&E-stained sections were made using a digital Spot camera mounted on an Olympus microscope. Immunofluorescence microscopy was performed with rabbit polyclonal antibody against TRPA1 without or with a blocking peptide of the TRPA1 antibody and Alexa Fluor 647 goat anti-rabbit secondary antibody (1:400 dilution; Invitrogen; Cat. \#: 21244). Slides were covered with DAPI containing Slow Fade® Gold anti-fade reagent (Invitrogen; Cat. \#: S36938), and fluorescence was visualized on a Nikon eclipse Ti fluorescence Microscope using NIS-Elements (Nikon; Japan) at 200X. A DAPI filter was used for nuclear staining and a Cy5 Red 
filter for TRPA1 staining (D. J. Conklin, Y. Guo, et al., 2019; Jin, Jagatheesan, et al., 2019).

\section{Statistics}

Data are presented as mean \pm standard error of mean (SEM). MannWhitney U Rank sum tests were used for the data comparison between two groups, and two-way Repeated Measures ANOVA with Bonferroni's post-test was used when comparing more than two groups with repeated measures (SigmaPlot, ver. 12.5; Systat Software, Inc., San Jose, CA). Statistical significance was set at $\mathrm{p}<0.05$.

\section{Results}

\section{Crotonaldehyde and Hemodynamics}

Non-invasive blood pressure and heart rate were measured in both air- and CR-exposed male mice once a week during the chronic study (Figs. 31A, B). Compared with the air-exposed control group, CR-exposed mice had significantly lower systolic, mean, and diastolic blood pressures at the $7^{\text {th }}, 8^{\text {th }}$, and $10^{\text {th }}$ weeks of exposure. Diastolic and mean blood pressures also were decreased significantly in the CR-exposed mice in the fifth week compared with the baseline measurements made during the second week of exposure (Fig. 31A). Heart rate was significantly higher in CR- than in air-exposed mice by echocardiography (under isoflurane anesthesia; Table 18). In addition to HR change, isovolumic relaxation time (IVRT) was significantly decreased in CR-exposed mice compared 
with air controls (Table 18). There was no effect, however, of chronic CR exposure on aortic stiffness as indexed by pulse wave velocity (PWV) measured by ultrasound (Table 18).

Chronic Exposure to Crotonaldehyde and Vascular Reactivity

Because chronic exposure to CR lowered blood pressure but not pulse wave velocity, we investigated potential acute CR-induced blood pressure changes. For this a naïve mouse with an indwelling blood pressure cannula in the aortic arch and acutely exposed to CR (1 ppm) for a 9-min session. Onset of CR exposure was followed by a delayed yet robust decreases in diastolic blood pressure $(15 \mathrm{~mm} \mathrm{Hg})$ and heart rate $(100 \mathrm{bpm})$ - both effects returned to baseline within 10 min after CR exposure ended and filtered air returned (Figs. 32 A, B). These data provide plausibility that even an acute and brief CR exposure at $1 \mathrm{ppm}$ can rapidly and reversibly depress systemic arterial blood pressure.

Although the aorta does not directly regulate blood pressure, the aorta is an important marker of systemic vascular dysfunction especially of endothelial dysfunction and atherosclerosis risk. CR had no effect on the efficacy of PE, ACh or SNP action in the aorta of chronically-exposed male mice (Table 19) and there also were no differences in aortic sensitivity or contractility to PE between exposure groups, however the ACh-induced, endothelium-dependent relaxations were significantly stronger (\% relaxation; Fig. 32A) and more potent (i.e., enhanced sensitivity; EC50 shifted to right; Fig. 32B) in the CR group vs air group (Table 20). By comparison, the endothelium-independent relaxations of SNP were not different between groups (Fig. 32C, D; Table 20) indicating a preferential CR 
effect on the endothelium. Interestingly, this effect may have contributed to the lower overall arterial blood pressure measured in the CR-exposed group compared with air group.

\section{Acute Exposure to Crotonaldehyde and Vascular Reactivity}

Because of the endothelium-dependent changes seen in the aortas of chronic CR-exposed WT male mice, vascular function was also measured postexposure in isolated aorta of WT male mice after 4 days of CR exposure ( 1 and 3 ppm). In the aorta of CR-exposed mice after both 1 and $3 \mathrm{ppm}$, there was a significant increase in relaxation and a slight increase in sensitivity of aorta in response to ACh (Fig. 33A, B; Fig. 34A, B). Similarly but, modestly there was also an increased relaxation (Fig. 33C; Fig. 34C) and sensitivity (Fig. 33D; Fig. 34D) in response to SNP that resulted in a significantly decreased $\mathrm{EC}_{50}$ (at 3 ppm only; Table 20).

Acute Exposure to Crotonaldehyde and Role of TRPA1

Because of the known relationship between the TRPA1 channel and neurogenic inflammation induced by MCS or acrolein, we performed acute exposures to CR (4 days; 1 ppm) in TRPA1-null male mice. In contrast to the WT mice, measures of vascular reactivity in TRPA1-null mice showed no significant changes in relaxation or sensitivity in response to ACh or SNP (Fig. 35), nor was there was a significant change in any $\mathrm{EC}_{50}$ (Table 20). These data indicate that the vascular responses observed in male WT mice in response to CR were likely mediated by TRPA1. 
Acute Exposure to Crotonaldehyde and sex dependence

Because acrolein-induced toxicity showed protective in female mice in our previous study. We performed acute exposures to CR (4 days; $1 \mathrm{ppm}$ ) in female mice. In contrast to the WT mice, measures of vascular reactivity in female mice showed no significant changes in relaxation or sensitivity in response to ACh or SNP (Fig. 36), nor was there was a significant change in any EC50 (Table 20). These data indicate that the vascular effects of $\mathrm{CR}$ at $1 \mathrm{ppm}$ were sex-dependent.

Acute Exposure to Formaldehyde or Acetaldehyde and Vascular Reactivity

We also measured vascular function in isolated aorta of WT male mice after 4 days of $\mathrm{FA}(5 \mathrm{ppm})$, there was no difference in the relaxation and the sensitivity in response to ACh or SNP (Fig. 37). Mice were exposed to 2 weeks of AA (5 ppm), there was no difference in the relaxation and the sensitivity in response to ACh or SNP (Fig. 38). These results indicated that there was limited vascular dysfunction after acute FA or sub-acute AA exposure.

Compare the vascular toxicity of aldehydes

The PE2/PE1 tension (\%) which was calculated by dividing PE-induced tension post-CR-exposure (PE2) by PE-induced tension pre-CR (i.e., PE1) and multiplied by 100 . The PE2/PE1 tension (\%) was significantly inhibited after CR exposure in vitro compared to FA or AA exposure (Fig. 39). The effective concentration inducing $50 \%$ relaxation (EC50) of CR-induced vasorelaxation was significantly lower than FA and AA in PE precontracted superior mesenteric artery (SMA) (Table 21). The unsaturated aldehyde (i.e., CR) caused more toxic to vascular 
smooth muscle than saturated aldehydes (i.e., FA and AA). However, both FA $(0 \sim 300 \mu \mathrm{M})$, AA $(0 \sim 30 \mathrm{mM})$ and $\mathrm{CR}(0 \sim 300 \mu \mathrm{M})$ showed limited endothelial dysfunction after exposure in vitro. 
Fig.31. Hemodynamic effects of inhaled crotonaldehyde (CR) in mice. Blood pressure was measured non-invasively by tail cuff method in C57BL/6J male mice weekly (within $1 \mathrm{~h}$ after exposure) during the chronic air and CR exposures (1 ppm; 6h/d, $5 \mathrm{~d} /$ week, 12 weeks) (A). Blood pressure ( $\mathrm{mm} \mathrm{Hg}$ ) was significantly lower in CR-exposed mice compared with air-exposed control mice over several weeks of the latter half of the exposure $(\mathbf{A})$, but there was no consistent change in heart rate (HR, bpm) throughout (B). Aortic arch blood pressure was measured via invasive

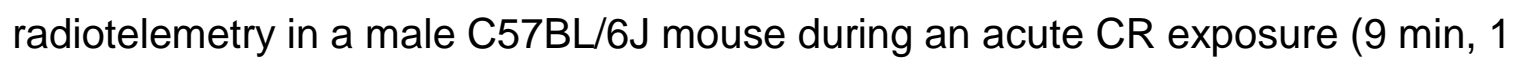
ppm). Diastolic blood pressure (DBP; C) and HR (D) fell rapidly upon onset of CR exposure, and both parameters recovered quickly to baseline levels within 10-12 min after ceasing CR exposure. Aortic arch blood pressure was measured via invasive radiotelemetry in male C57BL/6J mice during an acute CR exposure (3h, $1 \mathrm{ppm} ; 3 \mathrm{~h}, 3 \mathrm{ppm})$ to simulate our acute and chronic exposure conditions. Although only modest drops in DBP $(\mathbf{E})$ and HR $(\mathbf{F})$ were observed upon beginning $1 \mathrm{ppm}$ CR exposure, there were much more noticeable drops in DBP (E) and HR (F) when the CR level was increased to 3 ppm. As with brief CR exposure (9 min, $1 \mathrm{ppm}), \mathrm{HR}$ recovered quickly to baseline levels within 5 min of ceasing the CR exposure, however, DBP was slow to recover and did not reach baseline levels after 15 min. Summary data of two mice exposed to CR (1 and 3 ppm, 3h each) showed that all blood pressures (systolic, mean, diastolic; G) were suppressed equally by CR exposure whereas mean HR was not $(\mathbf{H})$. Values = mean \pm SE $(n=9$ 10 mice per group, A, B; $n=2, \mathbf{G}, \mathbf{H}) ; a, p<0.05$ vs air control; $b, p<0.05$ vs baseline. 

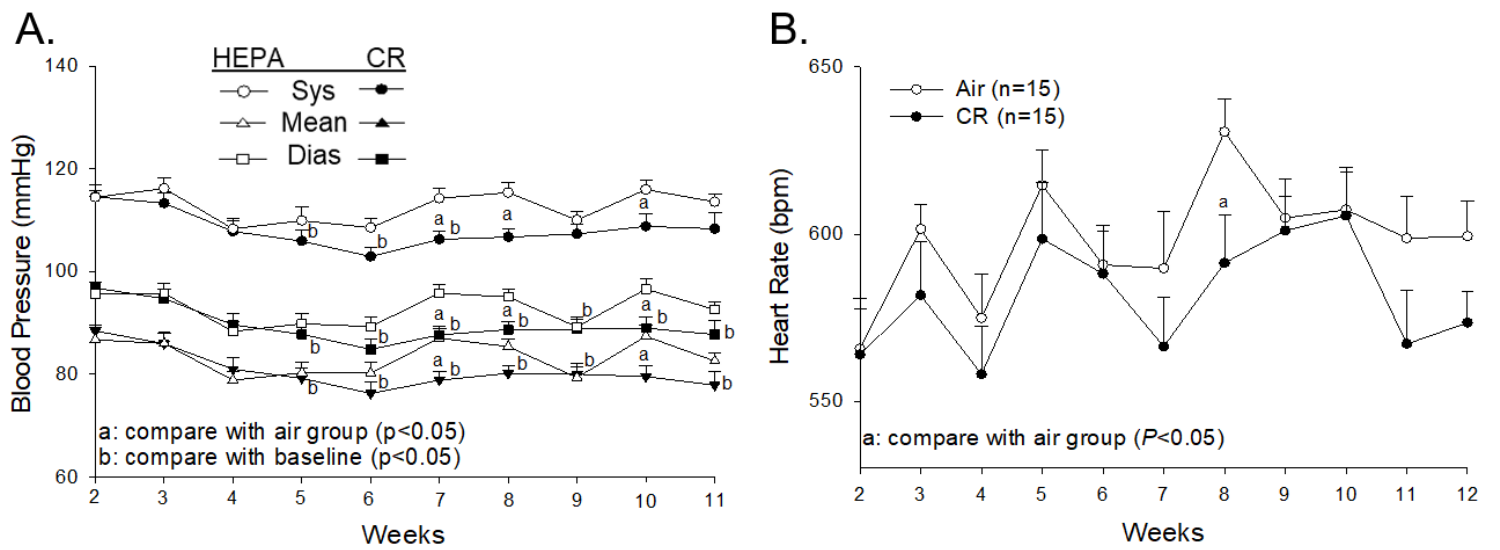

C.

D.
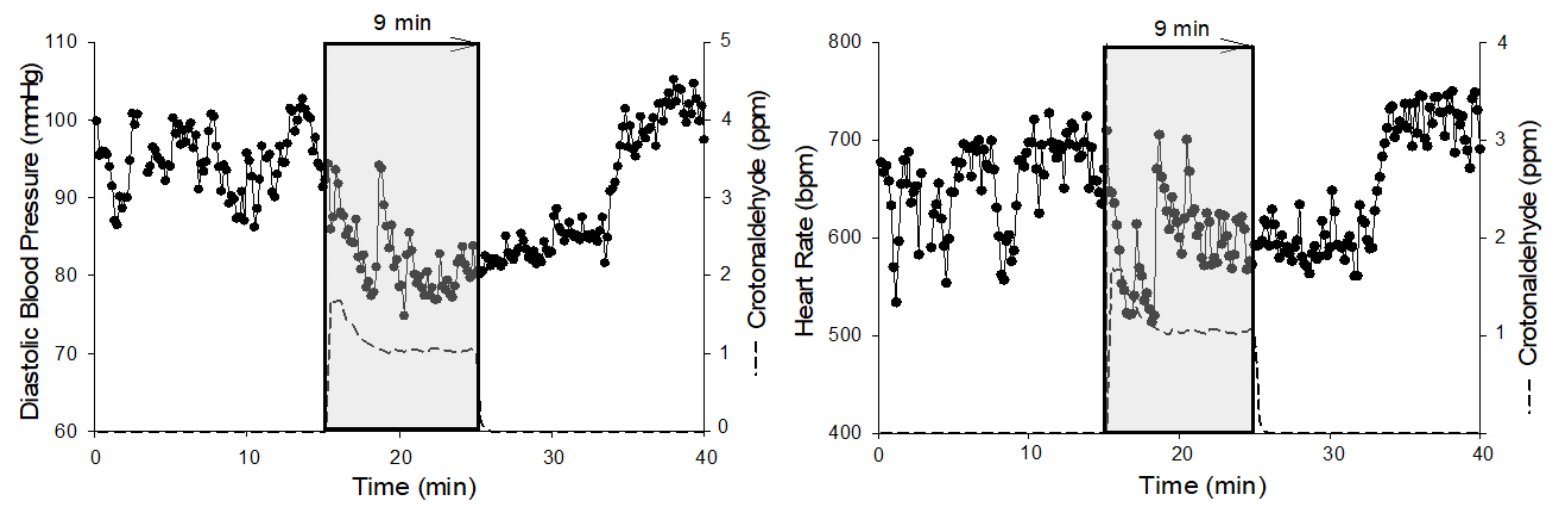
E.

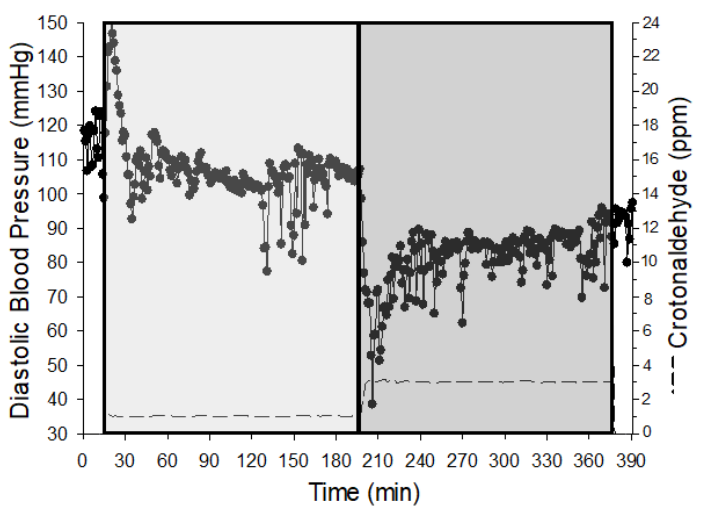

G.

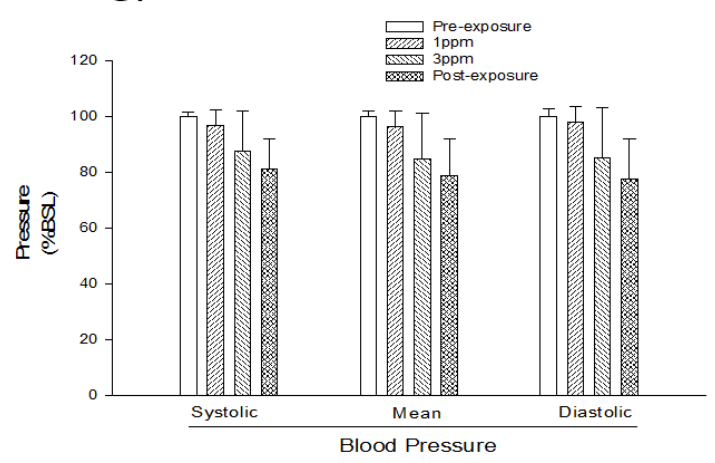

F.

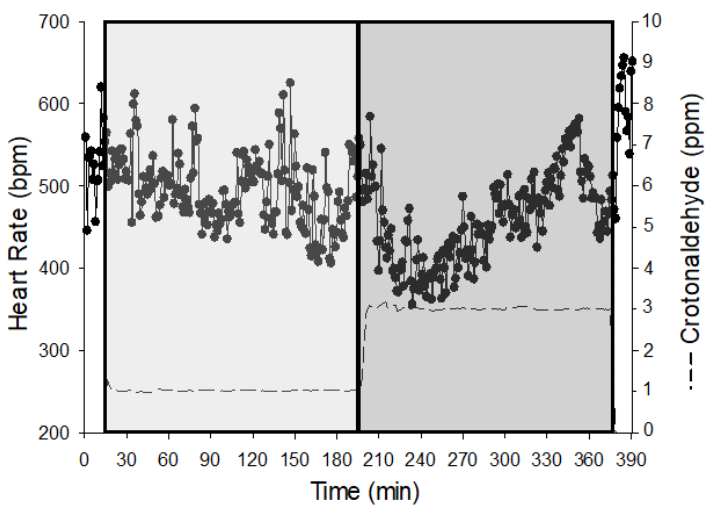

$\mathrm{H}$.

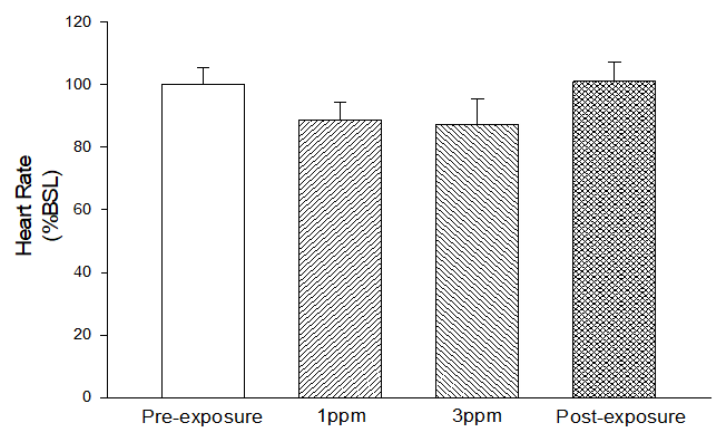


Table 18. Echocardiography measures in male mice chronically exposed to air or crotonaldehyde.

\begin{tabular}{|c|c|c|}
\hline \multirow[b]{2}{*}{ Measurement } & \multicolumn{2}{|c|}{ Exposure } \\
\hline & Air Control (5) & 1 ppm CR (5) \\
\hline Body Weight (g) & $29.1 \pm 0.5$ & $28.6 \pm 0.5$ \\
\hline HR (bpm) & $476 \pm 20$ & $536 \pm 13^{*}$ \\
\hline EDV $(\mu \mathrm{L})$ & $42 \pm 7$ & $41 \pm 2$ \\
\hline ESV $(\mu \mathrm{L})$ & $12 \pm 2$ & $11 \pm 1$ \\
\hline SV $(\mu \mathrm{L})$ & $30 \pm 4$ & $30 \pm 2$ \\
\hline EF (\%) & $72 \pm 1$ & $74 \pm 2$ \\
\hline $\mathrm{CO}(\mathrm{mL} / \mathrm{min})$ & $13.9 \pm 1.4$ & $16.2 \pm 0.7$ \\
\hline LVIDd (mm) & $3.7 \pm 0.1$ & $3.4 \pm 0.1$ \\
\hline LVIDs (mm) & $2.1 \pm 0.1$ & $1.9 \pm 0.2$ \\
\hline LVPWd (mm) & $0.8 \pm 0$ & $1.0 \pm 0.1$ \\
\hline LVPWs (mm) & $1.3 \pm 0.1$ & $1.4 \pm 0.1$ \\
\hline LVAWd (mm) & $1.1 \pm 0.1$ & $1.1 \pm 0$ \\
\hline LVAWs (mm) & $1.6 \pm 0.1$ & $1.6 \pm 0$ \\
\hline LVM (mg) & $105.4 \pm 6.9$ & $103.6 \pm 6.8$ \\
\hline IVRT (ms) & $13.3 \pm 0.9$ & $9.6 \pm 1.2^{*}$ \\
\hline Aortic AVG & $2587.0 \pm 698.8$ & $1806.8 \pm 71.0$ \\
\hline Innominate AVG & $1209.8 \pm 78.6$ & $1216.0 \pm 67.1$ \\
\hline LCC_AVG & $744.6 \pm 69.8$ & $770.8 \pm 95.4$ \\
\hline PWV (Right \& Left; mm/s) & $2.8 \pm 0.1$ & $2.9 \pm 0.1$ \\
\hline RWT (mm) & $0.46 \pm 0.03$ & $0.56 \pm 0.05$ \\
\hline
\end{tabular}

Values $=$ mean $\pm S E .(n)$, number of mice; ${ }^{*}, p<0.05$ compared to air control; \#, $0.05 \leq p \leq 0.10$ compared to air control. Abbr.: CO, cardiac output; CR, crotonaldehyde; EDV, end-diastolic volume; EF, ejection fraction; ESV, endsystolic volume; HR, heart rate; IVRT, isovolumic relaxation time; LCC, left coronary cusp; LVAWd, left ventricular anterior wall thickness at end-diastole; LVAWs, left ventricular anterior wall thickness at end-systole; LVIDd, left ventricular internal dimension at end-diastole; LVIDs, left ventricular internal dimension at end-systole; LVM, left ventricular mass; PWV, pulse wave velocity; RWT, relative wall thickness; SV, stroke volume. 
Fig. 32. Vascular toxicity of chronic crotonaldehyde (CR) inhalation exposure in mice. Aortic function was measured ex vivo in male C57BL/6J mice following 12 weeks of exposure to CR (1 ppm, 6h/d, $5 \mathrm{~d} /$ week, 12 weeks). Acetylcholine (ACh) was used to assess aortic endothelium-dependent relaxation efficacy (\% relaxation; A) and sensitivity ( $\mathrm{EC}_{50}$; $\left.\mathbf{B}\right)$ in phenylephrine $(\mathrm{PE})$-precontracted aortic rings by isometric myography. CR exposure significantly enhanced ACh sensitivity (B). There were, however, no changes in aortic efficacy (\% relaxation; C) and sensitivity $\left(\mathrm{EC}_{50} ; \mathrm{D}\right)$ to the endothelium-independent vasorelaxant sodium nitroprusside $(S N P)$. Values $=$ mean \pm SE $\left(n=10\right.$ mice per group); ${ }^{*}, p<0.05$ vs air control. 
A.

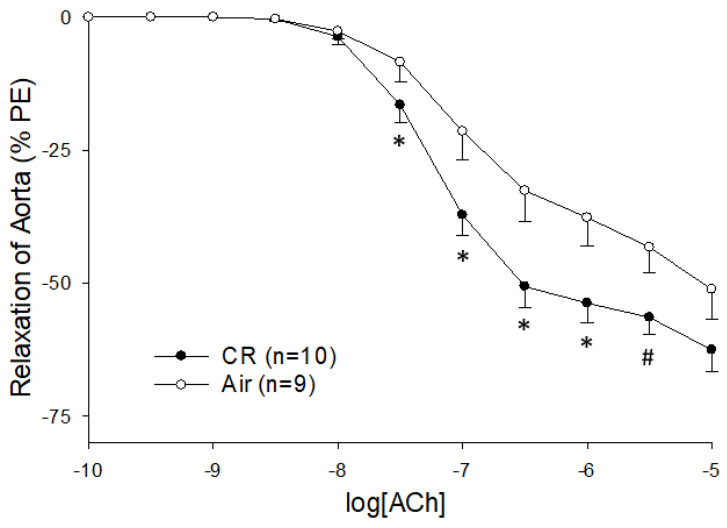

C.

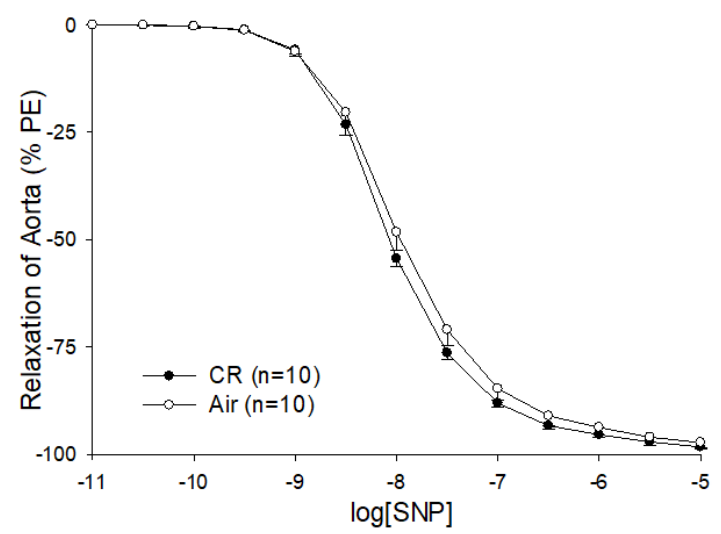

B.

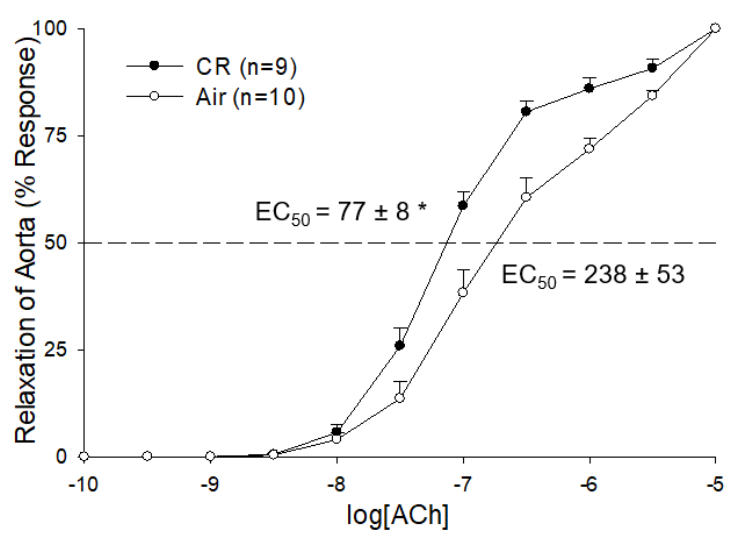

D.

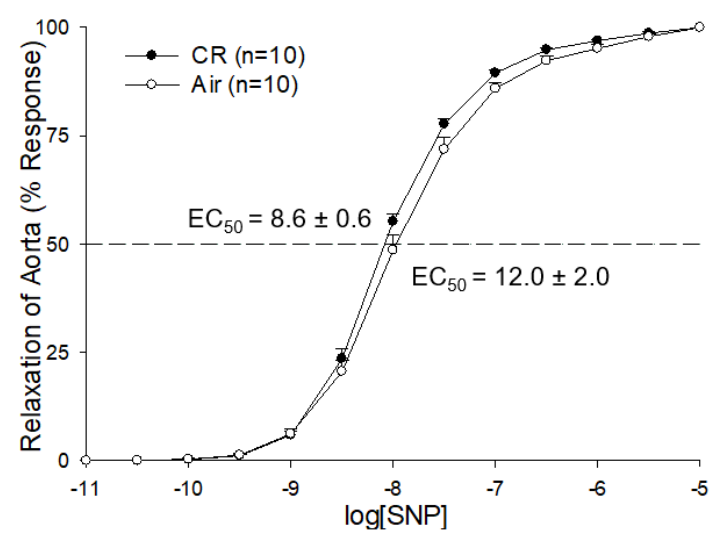


Fig. 33. Vascular toxicity of acute crotonaldehyde (CR) inhalation exposure in C57BL/6 mice. Aortic function was measured ex vivo following 4 days of exposure of male C57BL/6J mice to either air or CR (1 ppm, 6h/d, 4 days). Acetylcholine (ACh) was used to assess aortic endothelium-dependent relaxation efficacy (\% relaxation; $A)$ and sensitivity $\left(E_{50} ; \mathbf{B}\right)$ in phenylephrine (PE)precontracted aortic rings by isometric myography. CR exposure significantly enhanced ACh efficacy (A) and slighted shifted sensitivity (B). There were, however, no changes in aortic efficacy (\% relaxation; C) and sensitivity $\left(\mathrm{EC}_{50} ; \mathbf{D}\right)$ to the endothelium-independent vasorelaxant sodium nitroprusside (SNP). Values $=$ mean $\pm S E$ ( $n=8-10$ mice per group); ${ }^{*}, p<0.05$ vs air control. \#, $0.05 \leq p \leq 0.10$ vs air control. 
A.

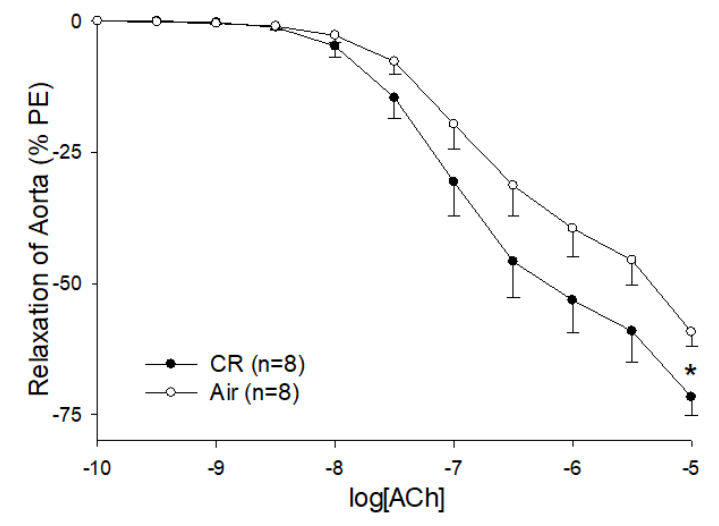

C.

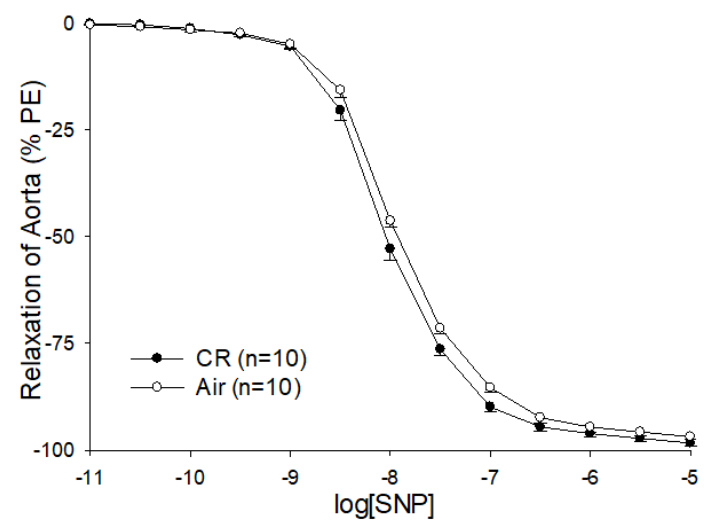

B.

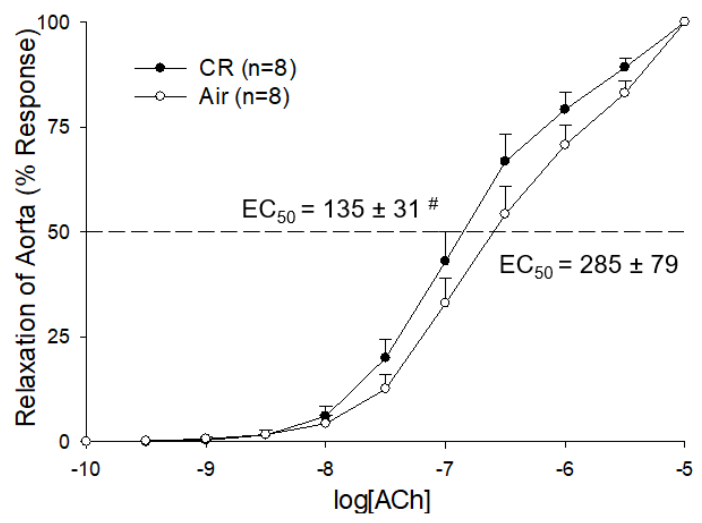

D.

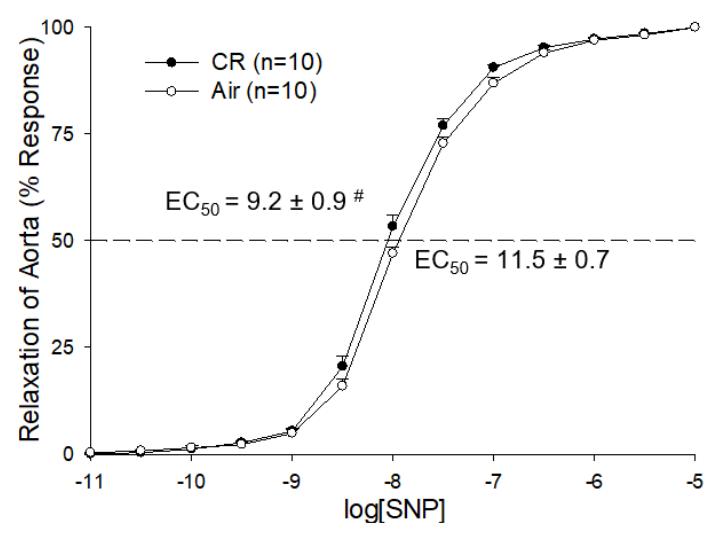


Fig. 34. Vascular toxicity of acute crotonaldehyde (CR) inhalation exposure in C57BL/6 mice. Aortic function was measured ex vivo following 4 days of exposure of male C57BL/6J mice to CR ( $3 \mathrm{ppm}, 6 \mathrm{~h} / \mathrm{d}, 4$ days). Acetylcholine (ACh) was used to assess aortic endothelium-dependent relaxation efficacy (\% relaxation; $\mathbf{A})$ and sensitivity $\left(\mathrm{EC}_{50} ; \mathbf{B}\right)$ in phenylephrine $(\mathrm{PE})$-precontracted aortic rings by isometric myography. CR exposure significantly enhanced ACh efficacy (A) and slighted shifted sensitivity (B). There was no change in aortic efficacy (\% relaxation; $\mathbf{C})$ but there was an enhanced sensitivity $\left(\mathrm{EC}_{50}\right.$; $\left.\mathbf{D}\right)$ in response to the endothelium-independent vasorelaxant sodium nitroprusside (SNP). Values = mean \pm SE ( $n=10$ mice per group); ${ }^{*}, p<0.05$ vs air control. \#, $0.05 \leq p \leq 0.10$ vs air control. 
A.

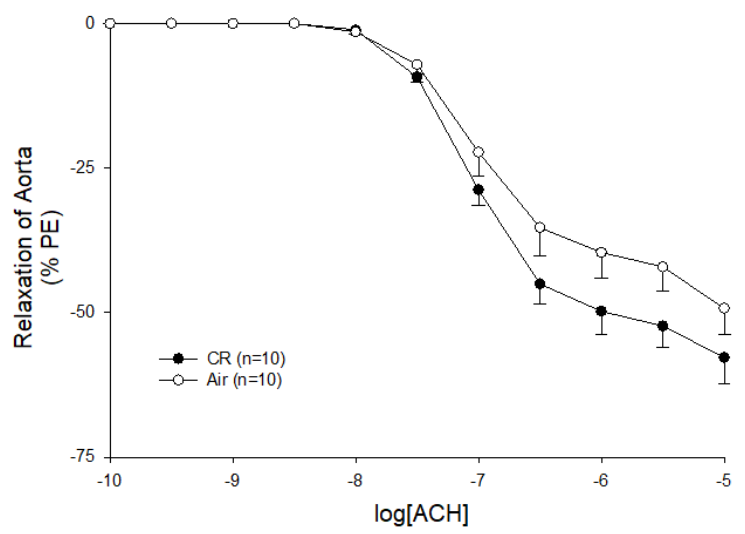

C.

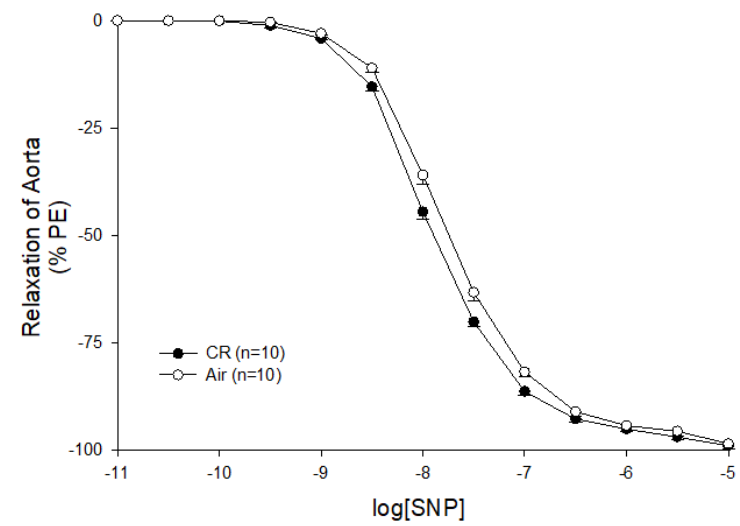

B.

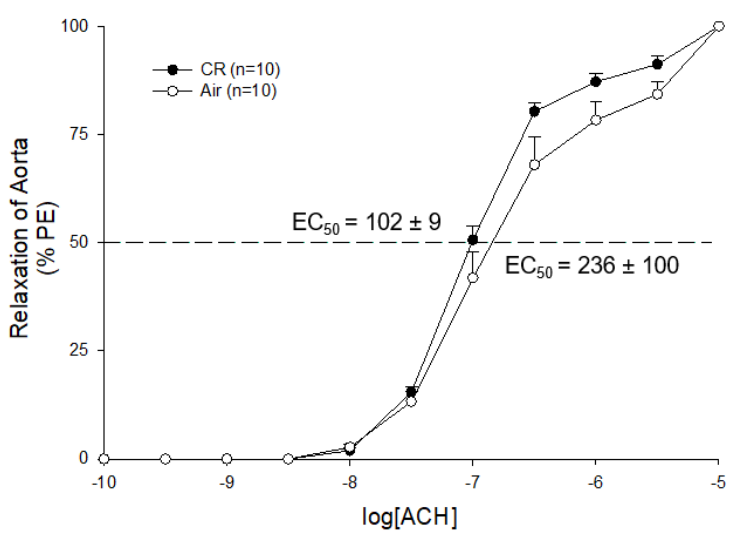

D.

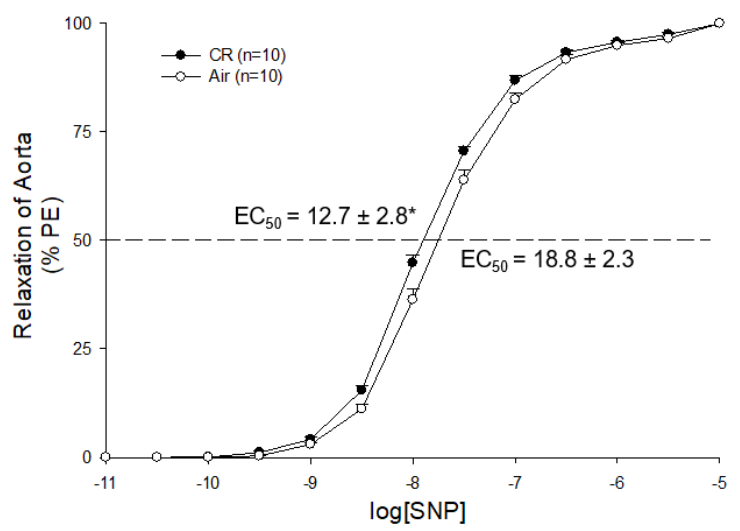


Table 19. Effects of either air or crotonaldehyde (CR; 1 or $3 \mathrm{ppm}$ ) exposure on efficacy responses of isolated thoracic aorta of C57BL/6J (wild type, WT) and TRPA1-null mice to pharmacological agents in the absence and presence of nitric oxide synthase inhibitor (L-NAME).

\begin{tabular}{|c|c|c|c|c|c|c|c|c|}
\hline \multicolumn{3}{|c|}{ Exposure Conditions } & \multicolumn{2}{|c|}{$\begin{array}{l}\text { PE, Emax } \\
{[\mathrm{mN} / \mathrm{mm}]}\end{array}$} & \multicolumn{2}{|c|}{ ACh, Emax [\%] } & & \\
\hline $\begin{array}{c}\text { Strain } \\
\text { (sex) }\end{array}$ & $\begin{array}{l}\text { Level } \\
\text { (ppm) }\end{array}$ & Duration & Air & CR & Air & CR & & \\
\hline WT & 1 & 4 days & $\begin{array}{l}4.1 \pm \\
0.7\end{array}$ & $\begin{array}{l}3.9 \pm \\
0.6\end{array}$ & $\begin{array}{c}-53.8 \pm \\
4.4\end{array}$ & $\begin{array}{l}-65.2 \\
\pm 5.2^{*}\end{array}$ & & \\
\hline $\begin{array}{c}\text { TRPA1- } \\
\text { null }\end{array}$ & 1 & 4 days & $\begin{array}{l}2.4 \pm \\
0.5\end{array}$ & $\begin{array}{l}2.1 \pm \\
0.3\end{array}$ & $\begin{array}{c}-64.6 \pm \\
3.4\end{array}$ & $\begin{array}{r}-59.8 \\
\pm 4.0 \\
\end{array}$ & & \\
\hline WT & 3 & 4 days & $\begin{array}{l}4.0 \pm \\
0.7\end{array}$ & $\begin{array}{l}3.7 \pm \\
0.7\end{array}$ & $\begin{array}{c}-49.3 \pm \\
4.6\end{array}$ & $\begin{array}{l}-57.8 \\
\pm 4.4\end{array}$ & & \\
\hline WT & 1 & $\begin{array}{c}12 \\
\text { weeks }\end{array}$ & $\begin{array}{l}3.2 \pm \\
0.6\end{array}$ & $\begin{array}{c}3.6 \pm \\
0.7 \\
\end{array}$ & $\begin{array}{c}-51.2 \pm \\
5.6\end{array}$ & $\begin{array}{l}-62.6 \\
\pm 3.8 \\
\end{array}$ & & \\
\hline WT (f) & 1 & 4 days & $\begin{array}{l}3.5 \pm \\
0.6\end{array}$ & $\begin{array}{l}4.0 \pm \\
0.6\end{array}$ & $\begin{array}{c}-76.4 \pm \\
3.8\end{array}$ & $\begin{array}{l}-77.4 \\
\pm 4.6\end{array}$ & & \\
\hline \multicolumn{3}{|c|}{ Exposure Conditions } & \multicolumn{2}{|c|}{$\begin{array}{l}\text { PE Ratio, +L- } \\
\text { NAME }\end{array}$} & \multicolumn{2}{|c|}{$\begin{array}{c}\text { ACh, [\%], +L- } \\
\text { NAME }\end{array}$} & \multicolumn{2}{|c|}{$\begin{array}{c}\text { SNP, [\%], +L- } \\
\text { NAME }\end{array}$} \\
\hline $\begin{array}{c}\text { Strain } \\
\text { (sex) }\end{array}$ & $\begin{array}{l}\text { Level } \\
\text { (ppm) }\end{array}$ & Duration & Air & CR & Air & CR & Air & CR \\
\hline WT & 1 & 4 days & $\begin{array}{l}1.3 \pm \\
0.03\end{array}$ & $\begin{array}{l}1.5 \pm \\
0.05^{\star}\end{array}$ & $\begin{array}{l}-1.4 \pm \\
0.5\end{array}$ & $\begin{array}{l}-0.9 \pm \\
0.5\end{array}$ & $\begin{array}{c}-96.9 \pm \\
0.7\end{array}$ & $\begin{array}{r}-98.4 \pm \\
0.6\end{array}$ \\
\hline $\begin{array}{c}\text { TRPA1- } \\
\text { null }\end{array}$ & 1 & 4 days & $\begin{array}{c}1.8 \pm \\
0.1\end{array}$ & $\begin{array}{c}2.0 \pm \\
0.2\end{array}$ & $\begin{array}{l}-1.7 \pm \\
1.1\end{array}$ & $0 \pm 0$ & $\begin{array}{c}-99.7 \pm \\
0.2\end{array}$ & $\begin{array}{r}-99.6 \pm \\
0.2\end{array}$ \\
\hline WT & 3 & 4 days & $\begin{array}{l}1.4 \pm \\
0.04\end{array}$ & $\begin{array}{l}1.5 \pm \\
0.04^{\#}\end{array}$ & $0 \pm 0$ & $0 \pm 0$ & $\begin{array}{c}-98.6 \pm \\
1.3\end{array}$ & $\begin{array}{r}-99.1 \pm \\
0.7\end{array}$ \\
\hline WT & 1 & $\begin{array}{c}12 \\
\text { weeks }\end{array}$ & $\begin{array}{l}1.5 \pm \\
0.1\end{array}$ & $\begin{array}{l}1.5 \pm \\
0.1\end{array}$ & $\begin{array}{l}-0.3 \pm \\
0.2\end{array}$ & $\begin{array}{l}-0.8 \pm \\
0.5\end{array}$ & $\begin{array}{c}-97.3 \pm \\
1.3\end{array}$ & $\begin{array}{r}-98.3 \pm \\
0.6\end{array}$ \\
\hline WT (f) & 1 & 4 days & $\begin{array}{c}1.5 \pm \\
0.1\end{array}$ & $\begin{array}{l}1.5 \pm \\
0.05\end{array}$ & $\begin{array}{c}-0.5 \pm \\
0.4\end{array}$ & $\begin{array}{l}-1.9 \pm \\
1.5\end{array}$ & $\begin{array}{c}-99.4 \pm \\
0.5\end{array}$ & $\begin{array}{c}99.3 \pm \\
0.3\end{array}$ \\
\hline
\end{tabular}

Values = mean \pm SE $(n=10-11$ mice per group); Abbr.: ACh, acetylcholine; CR, crotonaldehyde; EC50, half maximal effective concentration; PE, phenylephrine; SNP, sodium nitroprusside; TRPA1, transient receptor potential ankyrin 1; *, $p<0.05$ compared with air control based on Bonferroni' s or Dunn' s post-test; $\#, 0.05 \leqslant \mathrm{p} \leqslant 0.10$ compared with air control based on Bonferroni' $s$ or Dunn' $s$ post-test; $\dagger, p<0.05$ for WT vs TRPA1-null based on Dunn' s post-test; $\uparrow$, $0.05<\mathrm{p}<0.10$ for WT vs TRPA 1 -null based on Dunn' s post-test. 
Table 20. Effects of either air or crotonaldehyde (CR; 1 or $3 \mathrm{ppm}$ ) exposure on sensitivity responses of isolated thoracic aorta of C57BL/6J (wild type, WT) and TRPA1-null mice to pharmacological agents.

\begin{tabular}{|c|c|c|c|c|c|c|c|c|}
\hline \multicolumn{3}{|c|}{ Exposure Conditions } & \multicolumn{2}{|c|}{$\mathrm{PE}, \mathrm{EC}_{50}[\mathrm{nM}]$} & \multicolumn{2}{|c|}{$A C h, E_{50}[n M]$} & \multicolumn{2}{|c|}{$\begin{array}{c}\text { SNP, EC }_{50} \\
\text { [nM }^{1}\end{array}$} \\
\hline $\begin{array}{c}\text { Strain } \\
(\operatorname{sex})\end{array}$ & $\begin{array}{l}\text { Leve } \\
\text { I } \\
\text { (ppm }\end{array}$ & $\begin{array}{c}\text { Durati } \\
\text { on }\end{array}$ & Air & CR & Air & CR & Air & CR \\
\hline WT & 1 & 4 days & $\begin{array}{c}128 \pm \\
30\end{array}$ & $\begin{array}{c}201 \pm \\
55\end{array}$ & $\begin{array}{c}285 \pm \\
79\end{array}$ & $\begin{array}{c}135 \pm \\
31^{\#}\end{array}$ & $\begin{array}{c}11.5 \pm \\
0.7\end{array}$ & $\begin{array}{l}9.2 \pm \\
0.9^{\#}\end{array}$ \\
\hline $\begin{array}{c}\text { TRPA1- } \\
\text { null }\end{array}$ & 1 & 4 days & $\begin{array}{c}198 \pm \\
34\end{array}$ & $\begin{array}{c}252 \pm \\
61\end{array}$ & $\begin{array}{c}156 \pm \\
30\end{array}$ & $\begin{array}{c}296 \pm \\
124\end{array}$ & $\begin{array}{c}9.9 \pm \\
0.4\end{array}$ & $\begin{array}{c}9.9 \pm \\
0.5\end{array}$ \\
\hline WT & 3 & 4 days & $\begin{array}{c}139 \pm \\
31\end{array}$ & $\begin{array}{c}167 \pm \\
26\end{array}$ & $\begin{array}{c}236 \pm \\
100\end{array}$ & $\begin{array}{c}102 \pm \\
9\end{array}$ & $\begin{array}{c}18.8 \pm \\
2.3\end{array}$ & $\begin{array}{c}12.7 \pm \\
2.8^{\star}\end{array}$ \\
\hline WT & 1 & $\begin{array}{c}12 \\
\text { weeks }\end{array}$ & $\begin{array}{c}108 \pm \\
26\end{array}$ & $\begin{array}{c}231 \pm \\
109\end{array}$ & $\begin{array}{c}238 \pm \\
53\end{array}$ & $\begin{array}{c}78 \pm \\
8^{*}\end{array}$ & $\begin{array}{c}12.0 \pm \\
2.0\end{array}$ & $\begin{array}{c}8.6 \pm \\
0.6\end{array}$ \\
\hline WT (f) & 1 & 4 days & $\begin{array}{c}157 \pm \\
45\end{array}$ & $\begin{array}{c}234 \pm \\
70\end{array}$ & $\begin{array}{c}100 \pm \\
15\end{array}$ & $\begin{array}{c}68 \pm \\
13\end{array}$ & $\begin{array}{c}8.5 \pm \\
1.9\end{array}$ & $\begin{array}{l}7.5 \pm \\
1.0\end{array}$ \\
\hline
\end{tabular}

Values = mean \pm SE ( $n=9-11$ mice per group); all values are of male mice except where (f) $=$ female mice. ${ }^{1}$, SNP EC 50 measured in presence of L-NAME, $100 \mu \mathrm{M}$. Abbr.: ACh, acetylcholine; CR, crotonaldehyde; $\mathbf{E C}_{50}$, half maximal effective concentration; PE, phenylephrine; SNP, sodium nitroprusside; TRPA1, transient receptor potential ankyrin 1; *, p $<0.05$ compared with air control based on Bonferroni's or Dunn's post-test; \#, $0.05 \leq \mathrm{p} \leq 0.10$ compared with air control based on Bonferroni's or Dunn's post-test. 
Fig. 35. Vascular toxicity of acute crotonaldehyde (CR) inhalation exposure in TRPA1-null mice. Aortic function was measured ex vivo following 4 days of exposure of male TRPA1-null mice to CR (1 ppm, 6h/d, 4 days). Acetylcholine (ACh) was used to assess aortic endothelium-dependent relaxation efficacy (\% relaxation; $\mathbf{A})$ and sensitivity $\left(\mathrm{EC}_{50}\right.$; $\left.\mathbf{B}\right)$ in phenylephrine $(\mathrm{PE})$-precontracted aortic rings by isometric myography. CR exposure had no effect on efficacy (A) or sensitivity (B) of ACh-induced relaxations. Similarly, there were no CR-induced changes in aortic efficacy (\% relaxation; C) or sensitivity $\left(\mathrm{EC}_{50} ; \mathbf{D}\right)$ in response to the endothelium-independent vasorelaxant sodium nitroprusside (SNP). Values = mean $\pm S E(n=10-11$ mice per group). 
A.

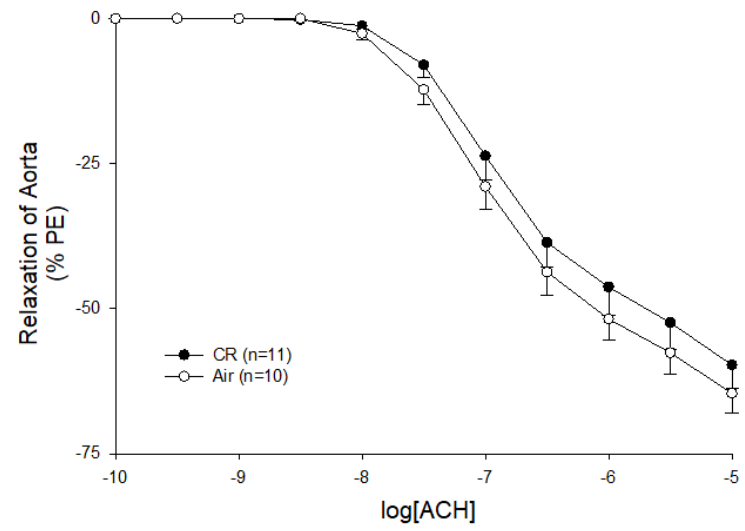

C.

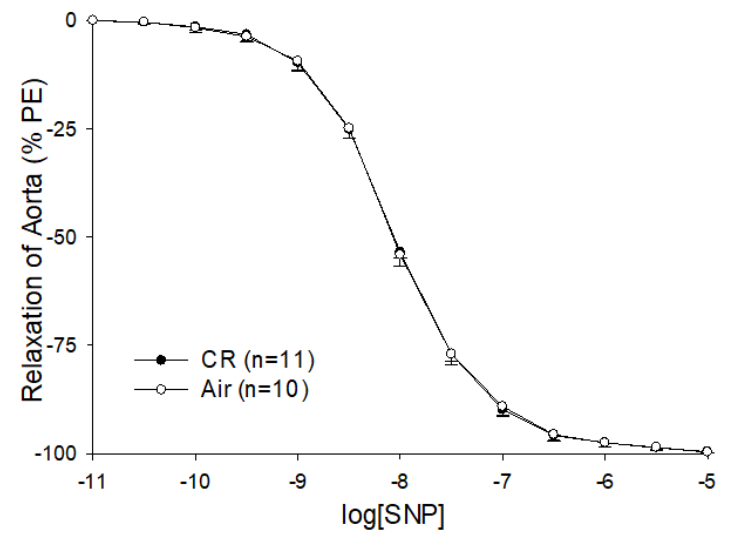

B.

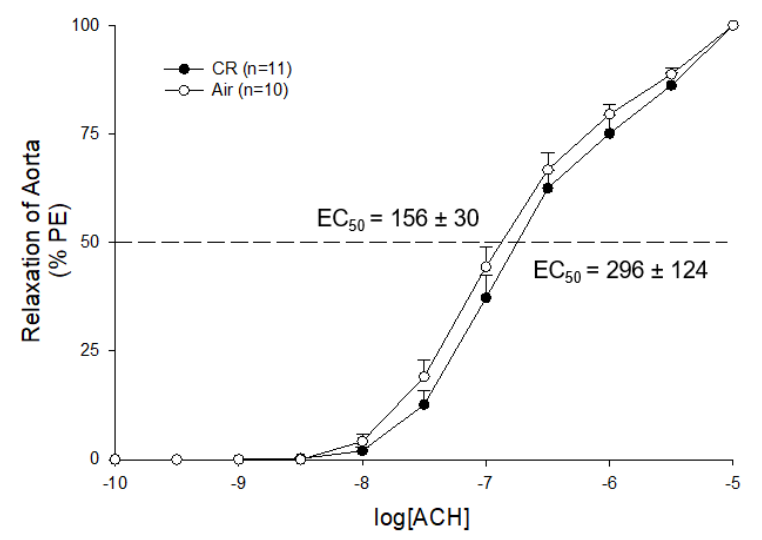

D.

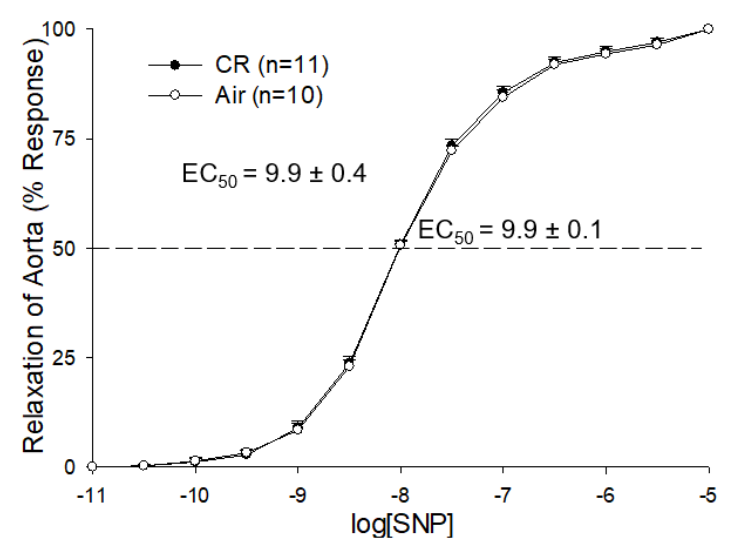


Fig. 36. Vascular toxicity of acute crotonaldehyde (CR) inhalation exposure female mice. Aortic function was measured ex vivo following 4 days of exposure of female mice to $\mathrm{CR}$ (1 ppm, 6h/d, 4 days). Acetylcholine (ACh) was used to assess aortic endothelium-dependent relaxation efficacy (\% relaxation; A) and sensitivity $\left(\mathrm{EC}_{50}\right.$; $\left.\mathbf{B}\right)$ in phenylephrine $(\mathrm{PE})$-precontracted aortic rings by isometric myography. CR exposure had no effect on efficacy (A) or sensitivity (B) of AChinduced relaxations. Similarly, there were no CR-induced changes in aortic efficacy (\% relaxation; C) or sensitivity $\left(\mathrm{EC}_{50} ; \mathrm{D}\right)$ in response to the endotheliumindependent vasorelaxant sodium nitroprusside (SNP). Values $=$ mean $\pm S E(n=9$ 11 mice per group). 
A.

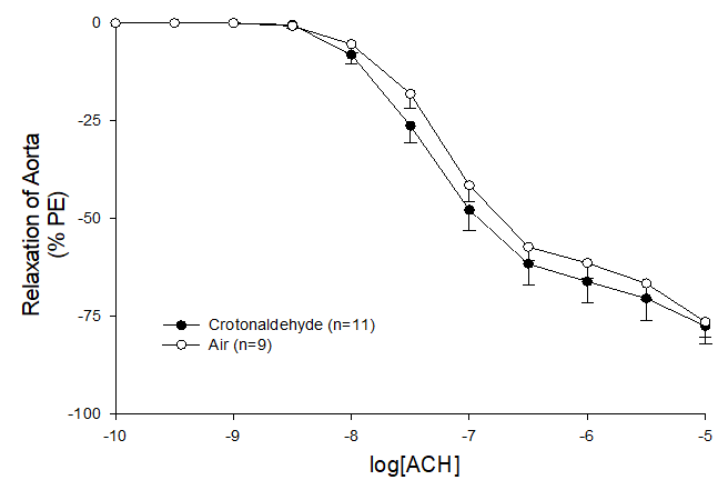

C.

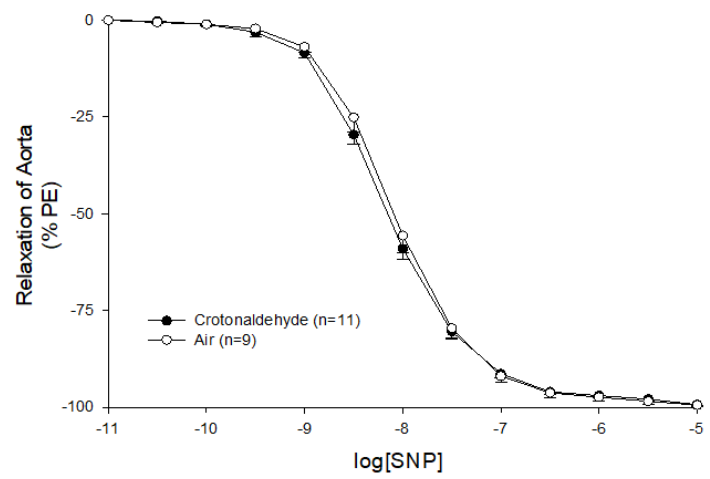

B.

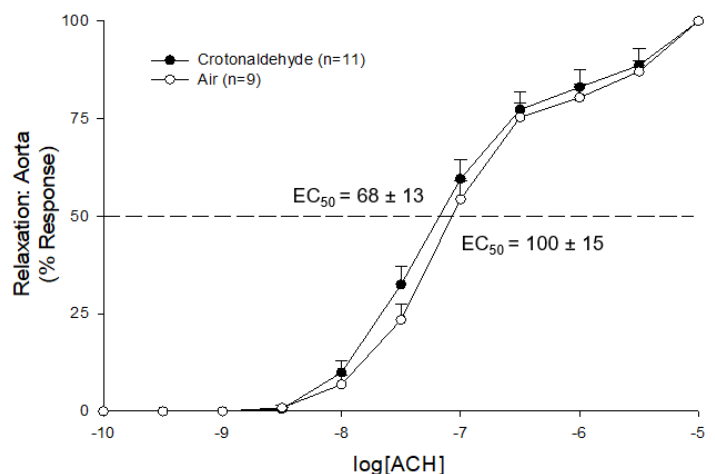

D.

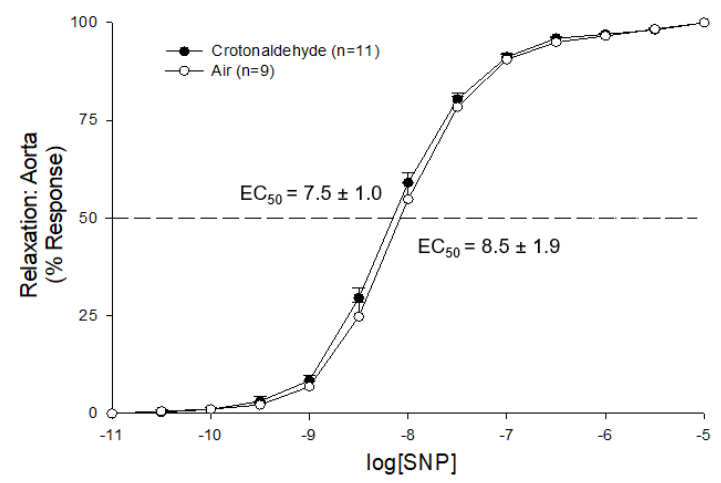


Fig. 37. The changes of aorta function after 4 days of exposure of FA $5 \mathrm{ppm}$. A) The relaxation of aorta to ACh. B) The relaxation of aorta to SNP. C) The sensitivity of aorta to ACh. D) The sensitivity of aorta to SNP. ${ }^{*}, P<0.05$ vs Control. 
A.

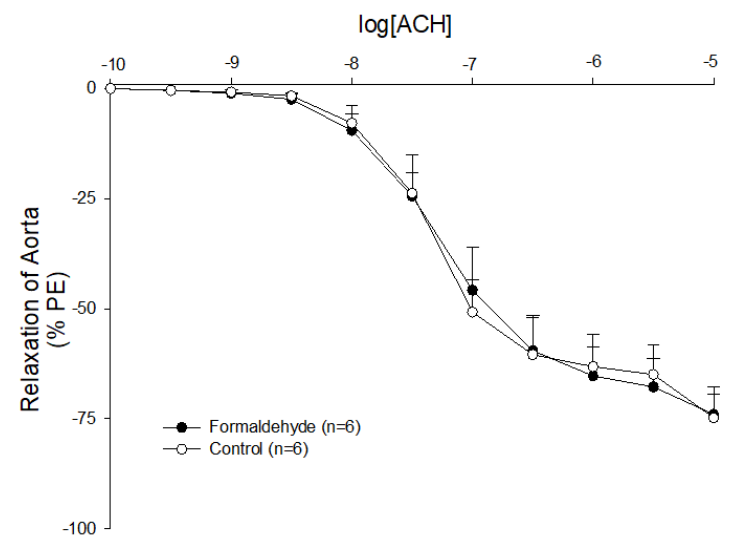

C.

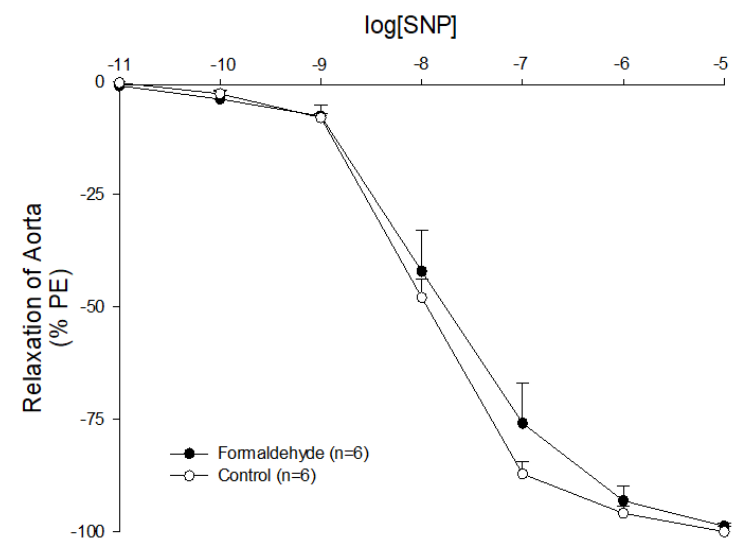

B.

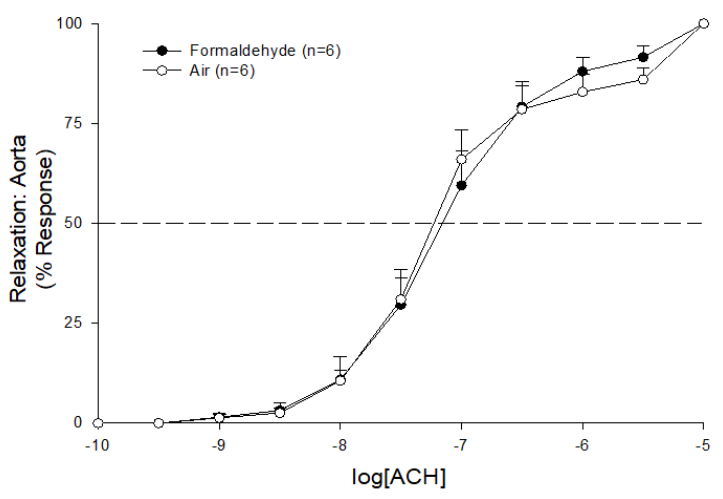

D.

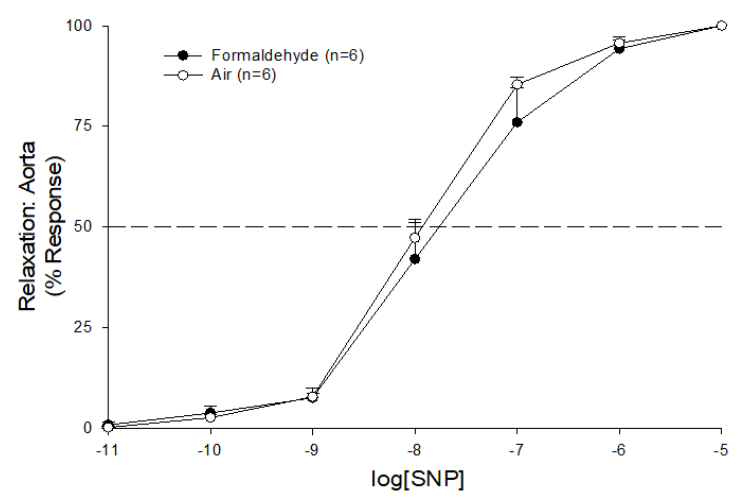


Fig. 38. The changes of aorta function after 2 weeks of exposure of AA $5 p p m$. A) The relaxation of aorta to $\mathrm{ACH}$. B) The relaxation of aorta to SNP. C) The sensitivity of aorta to ACH. D) The sensitivity of aorta to SNP. *, $\mathrm{P}<0.05$ vs Control. 
A.

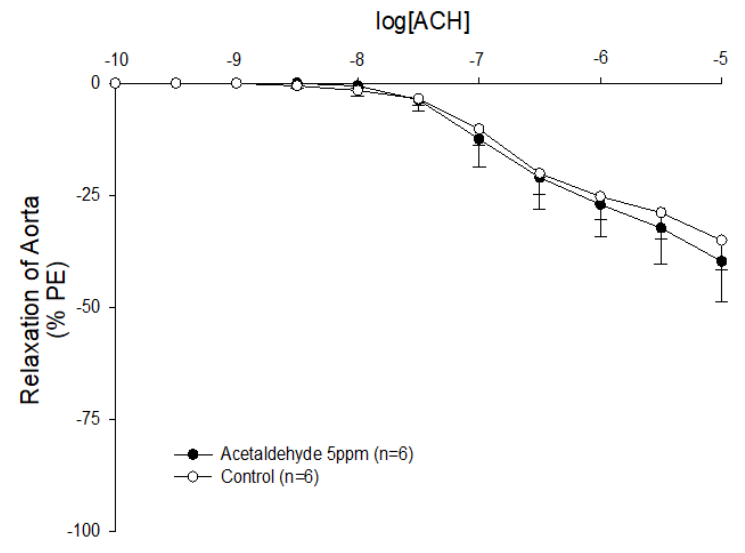

C.

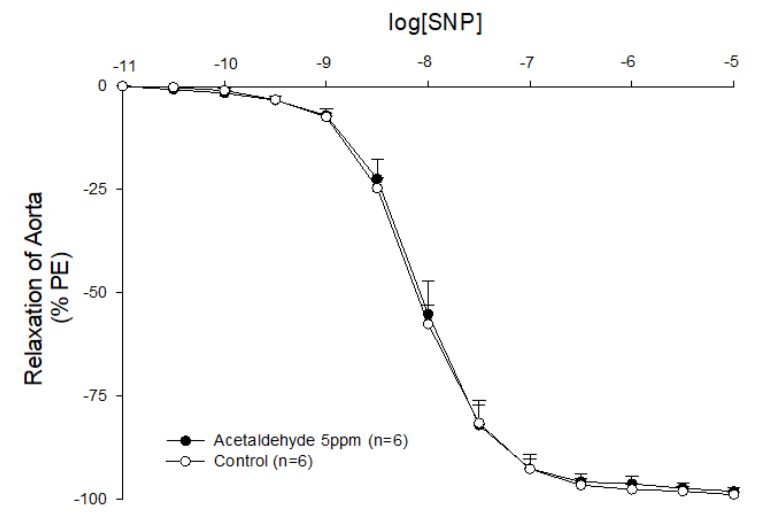

B.

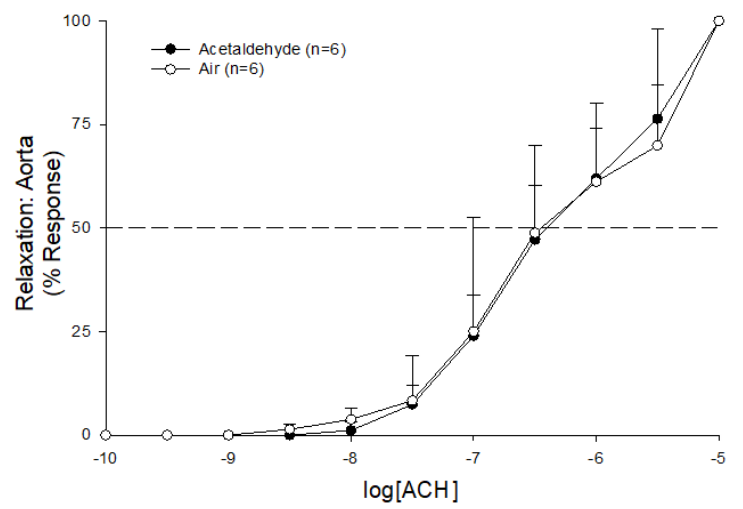

D.

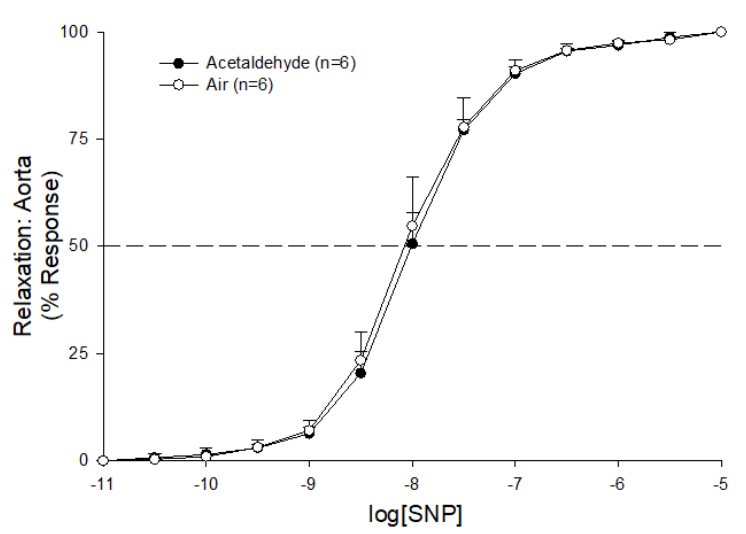




\section{Discussion}

Aldehydes are ubiquitous pollutants and toxic. Extensive epidemiological and experimental data show that aldehyde affect cardiovascular system. However, the processes by which aldehydes impair cardiovascular function and accelerate the cardiovascular disease are not known. This study especially show the vascular toxicity of CR in vivo and the possible mechanisms that would help us to study the toxicity of tobacco smoking and environmental pollution, and could also help government regulate the policies.

Crotonaldehyde is a hazardous air pollutant of significant human concern, especially as a component of cigarette smoke (IARC., 1995), which could induce apoptosis and necrosis in epithelial cells (Liu et al., 2010a, 2010b), oxidative stress, inflammation in alveolar macrophages (Facchinetti et al., 2007). In spite of these well-recognized toxicity of crotonaldehyde, the mechanisms underlying adverse effects of CR inhalation are not well understood. To our knowledge, this is the first study to evaluate the cardiovascular effects of CR exposure in vivo and propose that $\mathrm{CR}$ may contribute to vasodilation via a TRPA1- and endothelium-mediated pathway.

Isovolumic relaxation time (IVRT) is an interval in the cardiac cycle, from the aortic component of the second heart sound, that is, closure of the aortic valve, to onset of filling by opening of the mitral valve. The IVRT likely decreased with CR exposure because of the increased of heart rate or the decreased of the afterload as a result of low blood pressure (see Table. 18). We measure the blood pressure of the chronically-exposed mice immediately following an exposure 
period once a week for the duration of the study. The blood pressure significantly decreased in CR group compared with air group, although there was no difference in heart rate between these two groups (see Fig. 32); this might be the reason of decreased IVRT. Many studies have showed that shear stress is critically important in regulating the vascular physiology and pathobiology of the vessel wall by modulating endothelial cell (EC) function (C. Cheng et al., 2004; Malek, Alper, \& Izumo, 1999). In particular, low and oscillatory shear stress, but not increased shear stress, contributes to the development of atherosclerotic lesions (C. Cheng et al., 2006). Shear stress and stretch could also modulate EC functions by activating mechano-sensors and signaling pathways. Low shear stress in vitro can downregulate endothelial nitric oxide synthase (eNOS) and upregulate endothelin1 [Ziegler et al., 1998] and adhesion factors (Chappell, Varner, Nerem, Medford, \& Alexander, 1998), which results in an increase in the adhesion of monocytes. Of course, strong irritation in the airways can cause airway hyperresponsiveness and respiratory depression, and which are associated with systemic hypotension, though hypotension may also be caused by systemic cardiovascular depression due to parasympathetic vagal reflexes (B. P. Lee, Morton, \& Lee, 1992; Polverino et al., 2012).

Regardless of the mechanism, coronary vasospasm or peripheral vasodilation can cause ischemia and heart attack or hypotension and syncope, and/or even death and thus could be responsible for the drop in blood pressure after $\mathrm{CR}$ exposure. Furthermore, in isolated aorta of 4-day 3 ppm exposed mice, the sensitivity and relaxation of aorta to SNP increased significantly in CR group, 
and there was a trend that the sensitivity and relaxation of $\mathrm{ACH}$ also was increased in CR group (see Table 19; Fig. 34). We thus decreased the dose of CR to $1 \mathrm{ppm}$ to test for the effects at a lower level of exposure. In isolated aortas of 4-day and 12-week $1 \mathrm{ppm}$ exposed mice, the sensitivity and relaxation of aorta to ACh significantly increased in CR group. What's more, there was a trend that the sensitivity and relaxation of SNP increased in the exposed CR group after 4 days of exposure (see Table 19 and Fig. 32). These data show that the vasculature becomes more sensitive to both direct additions and chronic exposures to $\mathrm{CR}$, with the resulting effects being augmented smooth muscle relaxation. These consistent vascular effects indicate that chronic exposure to $\mathrm{CR}$ may lead to compensatory changes in the vasculature to offset excessive smooth muscle relaxation and hypotension.

Endothelium is important regulation of vascular tone through the production of a variety of mediators such as nitric oxide, reactive oxygen species, prostanoids, and endothelin. It not only acts as a barrier but also acts as an active signal transducer (Vita \& Keaney, 2002). Alteration in endothelial function precedes the development of morphological atherosclerotic changes and also contributes to the initiation and evolution of prothrombotic, proinflammatory and proliferative states. Endothelial dysfunction refers to impairment of endothelium-dependent vasodilatation and implies widespread abnormalities in endothelial integrity and homeostasis; this dysfunction might occur at an early stage of hypertension (Deanfield et al., 2007). Evaluation of endothelial function has been critical to our understanding of the significance of endothelium to cardiovascular system. 
Exposure to FA (5 ppm), AA (5 ppm) and CR 1 ppm showed limited endothelial effect in our study, but, CR exposure showed significant difference between WT mice and TRPA1-null mice.

TRPA1 is a cationic (calcium) channel and a promiscuous receptor concentrated in sensory fibers (unmyelinated C-fibers) that mediates pain reception/transmission (nociceptors). The distribution of TRPA1 receptors has expanded to non-neuronal system, such as heart, endothelium, urothelium and in the vasculature (Bautista et al., 2006; Bessac et al., 2008; Earley et al., 2009a; Sinharoy et al., 2017). Activation of peripheral sensory fiber TRPA1 leads to pain signaling and release of vasoactive peptides, substance $P$ (SubP) and cGRP (Russell, King, Smillie, Kodji, \& Brain, 2014; Trevisan et al., 2016), which may contribute to increased vasodilation and inflammatory response such as increased blood flow, increased vascular permeability (edema), increased white blood cell binding, and extravasation and pain (D. J. Conklin, 2016). TRPA1 is in the pulmonary airways and it plays a role in the protective physiological maneuver called "respiratory braking" elicited by inhaled toxins. Our previous study showed that TRPA1-null mice are more sensitive than WT mice to acrolein exposure and that TRPA1 antagonist injection post-acrolein exposure reduces acrolein-induced mortality (D. J Conklin et al., 2017; D. J. Conklin et al., 2017). This is consistent with the results that combined with TRPA1 antagonists (HC-030031) and TRPV1 antagonists (AMG 9810) can prevent pain and inflammation in pancreatic cancer mice (Schwartz et al., 2011). Interestingly, compared to the augmentation vasodilation in WT mice after CR exposure, there was no difference both in the 
sensitivity and relaxation of ACh and SNP in CR group and air group in TRPA1null mice after CR exposure (see Table 19 and Fig. 36). This disappearance of augmented smooth muscle relaxation in the vasculature indicate that TRPA1 plays an specific role in the toxicity of $\mathrm{CR}$. Future study still needs to make clear whether TRPA1 plays an protective function or induces impairment to the blood vessels.

The study of sex difference is very important in the exposure-related cardiovascular effects. Our previous study showed that female mice exposed to a high level of acrolein showed both pulmonary and systemic protection compared to the male mice, demonstrating reduced morbidity and a significantly lower sensitivity to acrolein-induced mortality (D. J Conklin et al., 2017). Although both acute and chronic exposure to $\mathrm{CR}$ induced vascular changes in male mice, females showed no changes in aortic sensitivity or reactivity in response to CR exposure in vivo (see Fig. 36). These differences between males and females post-CR exposure seem to support our previous findings that females are somewhat protected against $\alpha, \beta$-unsaturated aldehyde-induced toxicity and injury.

An aldehyde is a compound containing a functional group with the structure $-\mathrm{CHO}$, which is a carbon double-bonded to oxygen. Numerous studies show that unsaturated aldehydes are more toxic than saturated aldehydes in Pharmacodynamics. Our study also show that the concentrations of aldehydes cause relaxation of SMA is much higher in saturated aldehydes than in unsaturated aldehydes in vitro (see Chapter III, IV, V). The PE2/PE1 tension (\%) was significantly inhibited after CR exposure compared to FA or AA exposure in vitro 
(see Fig. 39). The effective concentration inducing 50\% relaxation (EC50) of CRinduced vasorelaxation was significantly lower than FA and $A A$ in PE precontracted superior mesenteric artery (SMA) (see Table 22). CR induce more toxic to vascular smooth muscle than FA or AA. However, both FA $(0 \sim 300 \mu \mathrm{M})$, AA $(0 \sim 30 \mathrm{mM})$ and $\mathrm{CR}(0 \sim 300 \mu \mathrm{M})$ didn't cause endothelial dysfunction after exposure in vitro. Future studies are needed to show the mechanism and pathology of aldehydes-induced endothelial dysfunction in vasculature.

In conclusion, this study indicates that chronic exposure to CR may lead to compensatory changes in the vasculature to offset excessive smooth muscle relaxation and hypotension that may lead to vascular disease. Because the vascular changes were not seen in TRPA1-null or in female WT mice, we conclude that these CR-induced changes within the vasculature are TRPA1- and sexdependent. However, additional studies are needed to further clarify these findings as well as to provide a better mechanistic understanding of CR-induced cardiovascular pathophysiology. As exposure to CR through both ambient and personal means continues to be a potential health threat to the global population, our current results provide novel insights into the cardiovascular effects of $C R$ and implicate chronic low levels of exposures as a potential inducer of CVD. Future studies are required to test the mechanisms and the toxicology of aldehydes. 
Table 21. The effective concentration inducing $50 \%$ relaxation (EC50) of aldehydeinduced vasorelaxation in isolated murine (male) superior mesenteric artery (SMA) with different contractile agonists in the absence and presence of the nitric oxide synthase (NOS) inhibitor, LNAME.

\begin{tabular}{|c|c|c|c|c|c|c|}
\hline \multirow{2}{*}{$\begin{array}{c}\text { EC50 } \\
(\mathrm{SMA})\end{array}$} & \multicolumn{2}{|c|}{ PE $\left(10^{-5} \mathrm{M}\right)$} & \multicolumn{2}{c|}{ U46619(10-7M) } & \multicolumn{2}{c|}{ High K+(60mM) } \\
\cline { 2 - 7 } & & +L-NAME & & +L-NAME & & +L-NAME \\
\hline $\begin{array}{c}\text { Formaldehyde } \\
(\mu \mathrm{M})\end{array}$ & $52.4 \pm 13.9$ & $206.9 \pm 10.4$ & $514.5 \pm 74.4$ & $568.9 \pm 56.6$ & $1093.2 \pm 50.4$ & $984.1 \pm 52.8$ \\
\hline $\begin{array}{c}\text { Acetaldehyde } \\
(\mathrm{mM})\end{array}$ & $3.3 \pm 0.3$ & $6.2 \pm 0.6$ & $14.9 \pm 1.5$ & $17.2 \pm 0.2$ & $17.5 \pm 0.5$ & $18.4 \pm 0.4$ \\
\hline $\begin{array}{c}\text { Crotonaldehyde } \\
(\mu \mathrm{M})\end{array}$ & $3.8 \pm 0.5$ & $43.9 \pm 17.7$ & & & & \\
\hline $\begin{array}{c}\text { Cinnamaldehyd } \\
\mathrm{e} \\
(\mu \mathrm{M})\end{array}$ & $11.9 \pm 3.1$ & $327.6 \pm 96.4$ & & & & \\
\hline $\begin{array}{c}\text { AITC } \\
(\mu \mathrm{M})\end{array}$ & $3.9 \pm 1.0$ & & & & & \\
\hline
\end{tabular}


Fig.39. Vascular smooth muscle toxicity of aldehydes (A) and endothelium function change after aldehydes exposure (B). PE, phenylephrine; the PE2/PE1 tension (\%) was calculated by dividing PE-induced tension post-CR-exposure (PE2) by PE-induced tension pre-CR (i.e., PE1) and multiplied by 100. 
A.

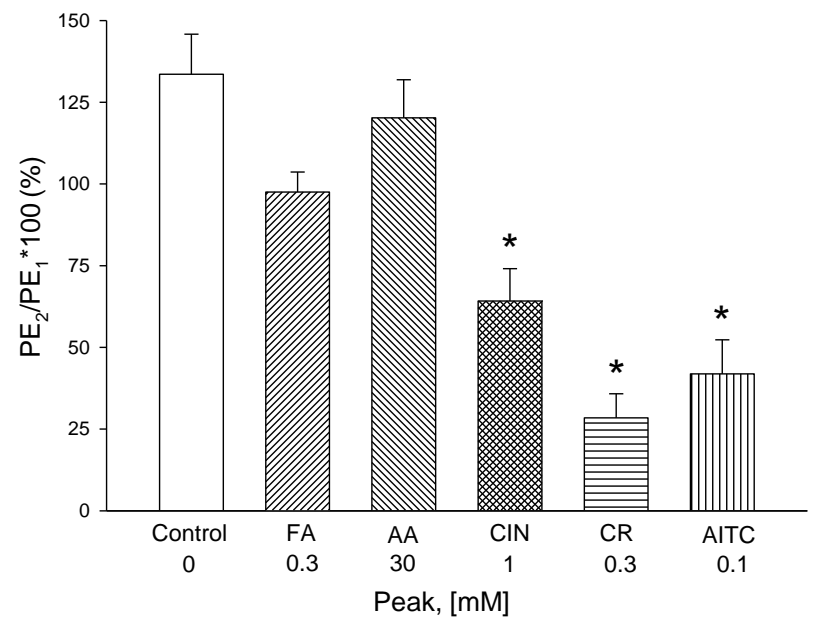

B.

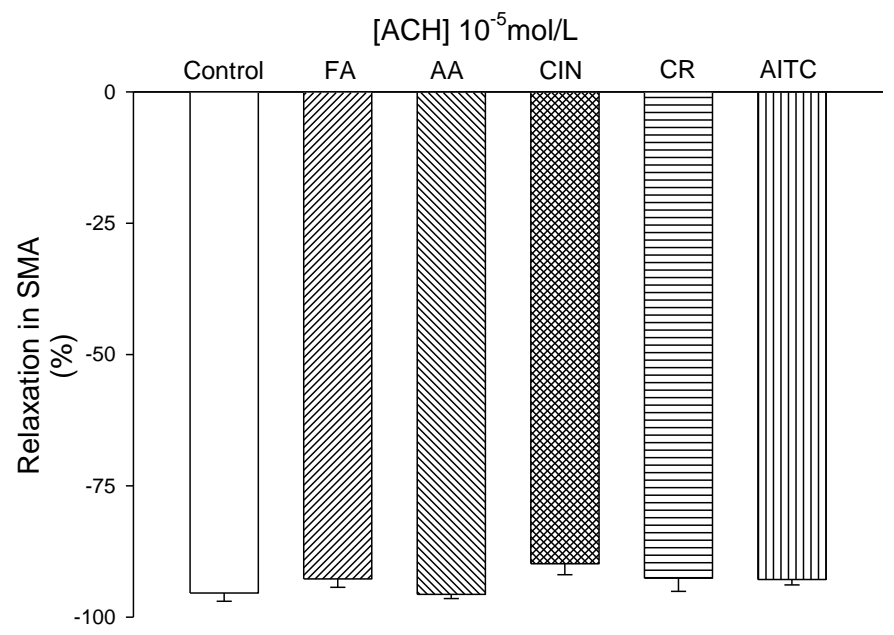




\section{CHAPTER VII}

\section{SUMMARY AND CONCLUSIONS}

\section{A. Major findings in this dissertation}

\section{Aldehydes exposure and vascular response in vivo and in vitro}

Aldehydes could be delivered either via inhalation, oral gavage, or intravenous injections. All these pathways would increase the concentration of aldehydes in the blood, which cause vasorelaxation of the blood vessels, including the blood vessels in the gastrointestinal (GI) tract (D. J. Conklin et al., 2004; Jin, Jagatheesan, et al., 2019). Although the effects of aldehydes have been studied for several decades, the vascular effects of aldehydes are still unclear. This study mainly focuses on the vascular toxicity of inhaling aldehydes $(\mathrm{FA}, \mathrm{AA}$, and $\mathrm{CR})$ and the underlying mechanisms.

Mice were exposed to CR (1 ppm) for 12 weeks, in the isolated aortas of exposed mice, the efficacy and sensitivity of ACh significantly increased in PEprecontracted aortic rings by isometric myography (see Chapter VI, Fig. 32; Table 19). In isolated aortas of 4-days CR $1 \mathrm{ppm}$ exposed mice, CR exposure significantly enhanced Ach efficacy and slightly shifted sensitivity. However, no 
changes in aortic efficacy and sensitivity to SNP (see Chapter VI, Fig. 33; Table 19). These data show that the vasculature becomes more sensitive to both direct additions and chronic exposures to $\mathrm{CR}$, with the resulting effect being augmented smooth muscle relaxation. Acute exposure to CR $1 \mathrm{ppm}$ significantly decreased the blood pressure during CR exposure by telemetry, did not recover shortly after CR exposure stop; these results were very robust and consistent (see Chapter VI, Fig. 31). Our study showed that inhalation of low concentrations of CR might affect the function of smooth muscles and induce hypotension. Many studies showed that hypotension could affect the quality of life and also induce cardiovascular diseases, such as myocardial infarction, syncope, heart failure, etc. (Duschek, Hoffmann, \& Reyes Del Paso, 2017; Owens \& O'Brien, 1999; Shibasaki, Ogawa, Yamada, Ouchi, \& Akishita, 2018; Tsujimoto \& Kajio, 2018), mice might also be hurt by hypotension. Chronic exposure to CR may lead to compensatory changes in the vasculature to offset excessive smooth muscle relaxation and hypotension, which might promote the release of neuronal hormones to increase blood pressure that would cause disease. We could not rule out that inhalation of CR might induce cardiovascular disease after long exposure.

FA and AA are contained in cigarette smoke. Our previous study showed that the levels of aldehydes were high in cigarettes (Lorkiewicz et al., 2019; Schick et al., 2017). In our study, after acute exposure to FA or subacute exposure to $A A$, there were no differences in the sensitivity or relaxation of $A C h$ and SNP in response to isolated aortas between the control and exposed groups 
(see Chapter VI, Fig. 38). The effect of FA or AA exposure on endothelium function is very limited at this exposure level. Many studies showed that FA is a carcinogen and an irritant, and we could not exclude the possibility that cancer would present an earlier risk than cardiovascular impairment (Nielsen, Larsen, \& Wolkoff, 2017; Tyihak, Bocsi, Timar, Racz, \& Szende, 2001). However, the risks of chronic inhalation of FA or AA at the levels found in tobacco or e-cigarette aerosols are still unknown.

An aldehyde is a compound containing a functional group with the structure $-\mathrm{CHO}$, which is a carbon double-bonded to oxygen, and unsaturated aldehydes are more active than saturated aldehydes. Our study shows that the concentrations of aldehydes required to cause relaxation of SMA is much higher in saturated aldehydes than in unsaturated aldehydes in vitro (see Chapter III, IV, V); FA and AA relaxed agonist-induced tension by $>90 \%$ in SMA and aorta.

The PE2/PE1 tension (\%) was significantly inhibited after CR exposure compared to FA or AA exposure in vitro (see Chapter VI, Fig. 39A). The effective concentration inducing $50 \%$ relaxation $\left(\mathrm{EC}_{50}\right)$ of $\mathrm{CR}$-induced vasorelaxation was significantly lower than FA and AA in PE-precontracted SMA (see Chapter VI, Table 22). Future studies are needed to show the mechanism and pathology of aldehyde-induced toxicity in the vasculature. CR stimulated isometric tension oscillations at high concentrations $(\geqslant 300 \mu \mathrm{M})$ (see Chapter $\mathrm{V}$, Fig.23B), though FA and AA did not, the mechanism of the oscillations is thus 
unclear. It might be dependent on the toxicity since it only showed in unsaturated aldehydes.

In conclusion, this study indicates that $\mathrm{CR}$ is more toxic to vasculature in vitro than FA and AA. Chronic exposure to CR may lead to compensatory changes in the vasculature to offset excessive smooth muscle relaxation and hypotension that may lead to vascular disease. However, additional studies are needed to further clarify these findings as well as to provide a better mechanistic understanding of CR-induced cardiovascular pathophysiology. As exposure to CR through both ambient and personal means continues to be a potential health threat to the global population, our current results provide novel insights into the cardiovascular effects of CR and implicate chronic low levels of exposures as a potential inducer of CVD.

\section{Aldehydes and endothelium function}

It is well known that the endothelium is very important in the regulation of vascular tone in the blood vessels (Furchgott \& Zawadzki, 1980; Palmer, Ferrige, \& Moncada, 1987). Endothelial dysfunction refers to impairment of endotheliumdependent vasodilatation, which implies widespread abnormalities in endothelial integrity and homeostasis and might occur at an early stage of hypertension (Deanfield et al., 2007), and it is the precursor of atherosclerosis and inflammation (Vanhoutte, Shimokawa, Feletou, \& Tang, 2017; Versari, Daghini, Virdis, Ghiadoni, \& Taddei, 2009). Our previous study showed that acrolein 
feeding increased atherosclerotic lesion formation in the aorta (Srivastava et al., 2011), and 5ppm of acrolein derived from tobacco smoke induced endothelium dysfunction in GSTP-null mice (D. J. Conklin, Haberzettl, et al., 2009b).

In contrast to the idea that FA induces oxidative stress in endothelial cells (Yu, 1998), our data indicate that in our endothelial dysfunction model in vitro, endothelial dysfunction blocks only the physiological (sensitive) component of FA-induced vasorelaxation. We did not observe FA-, AA-, or CR-induced endothelial dysfunction even after exposing SMA to $1,000 \mu \mathrm{M} F A, 300 \mathrm{mM}$ AA or $300 \mu \mathrm{M}$ CR. The relaxation of ACh was not different from vehicle group after CR exposure (see ChapterVI, Fig. 39B). This is consistent with our previous study: in isolated human coronary artery bypass graft (CABG) blood vessels with endothelial dysfunction, FA still induces relaxation, but in a far less sensitive manner ( $E_{50}, 315 \pm 0 \mathrm{mM}$ ) (D. J. Conklin et al., 2004) than that present in healthy mice as seen in our study.

Acute exposure to FA or $\mathrm{CR}$, subacute exposure to $\mathrm{AA}$, and chronic exposure to CR showed limited endothelial dysfunction in the isolated aortas. This could because the length and dose of exposure were not enough to see an effect or because arteries other than the aorta are more sensitive to aldehydes exposure in vivo study. It is well known that the effect of aldehydes is time- and dose-dependent (D. J. Conklin et al., 2004). The velocity of blood flow in the aorta is so fast that the amount of time the aldehydes affect endothelial cells in aorta is limited, or the little damaged endothelial cells might be repaired very fast. 


\section{Aldehydes and postprandial hyperemia}

Our study showed that urinary levels of the metabolites of FA and AA increase with feeding such that FA and AA likely contribute to the physiological reflex of post-prandial hyperemia via SMA vasodilatation. The SMA is a major arterial blood supply to the GI tract, and it dilates postprandially in response to many stimuli e.g., nutrients, vago-vagal reflex, NO, and substance P (Chou \& Coatney, 1994; Lucchini et al., 1996). These aldehydes are often considered to be toxicants, but they are naturally abundant in food, water, and other beverages (Aldehydes, 1981; D. J. Conklin, P. Haberzettl, et al., 2011) and their abundant metabolic products make them plausible stimuli for increasing Gl blood flow. This is the first suggestion of these physiological roles; more research will be needed to establish these aldehydes as bona fide contributors to postprandial hyperemia in vivo.

\section{The mechanisms of vasodilation of aldehydes}

L-NAME, an NO inhibitor, totally blocked the relaxation of the aorta (about $90 \%$ ), but the relaxation of the SMA is much less (approximately 15\%) (data not shown). We conclude that endothelium-derived hyperpolarizing factor (EDHF) is the dominant mechanism in the relaxation of the SMA, which is consistent with a previous study showing that EDHF is much more important in the relaxation of smaller blood vessels than in large conduit vessels (Tomioka et al., 1999). 
Testing the function changes in SMA is therefore a good way to study the smaller blood vessels as well as resistance arteries. Although the aorta does not directly regulate blood pressure, the aorta is an important marker of systemic vascular dysfunction, especially of endothelial dysfunction and atherosclerosis risk. We thus test the changes of aorta function in our in vivo studies and explore the mechanisms of vasodilation in SMA in our in vitro studies. The mechanisms of the vascular effects of aldehydes have been studied for several decades, but the complex mechanisms of this phenomenon are only now becoming clear.

We show that there are 3 distinct pathways of FA- and CR-induced vasorelaxation in SMA and 2 distinct pathways of AA-induced vasorelaxation in SMA (see Chapter III, Fig. 15; Chapter IV, Fig. 22; Chapter V, Fig. 29) and while this is fascinating in its own right, we focused on the mechanism of the most sensitive, and thus, most likely physiological pathway. The sensitive FA-, AA- and CR-induced vasorelaxations require a functional endothelium, NOS (presumably eNOS), and guanylyl cyclase (GC/cGMP). The endothelial cells likely contribute to the most sensitive components, whereas the latter component is likely present in vascular smooth muscle cells (VSMC). We propose a model for each of these three aldehydes featuring a stepwise sequence of events that is quite typical of agonists that elicit EDRF/NO. However, we also cannot rule out the possibility that additional components in the vascular wall (perivascular nerves, SubP, CGRP, etc...) may contribute to the AA-induced relaxation (Nilius et al., 2012). 
The $\mathrm{EC}_{50}$ of $\mathrm{FA}, \mathrm{AA}$ and $\mathrm{CR}$ are $52.4 \pm 13.9 \mu \mathrm{M}, 3.3 \pm 0.3 \mathrm{mM}$ and $3.8 \pm$ $0.5 \mu \mathrm{M}$, respectively, which show that $\mathrm{CR}$ is much more toxic than $\mathrm{FA}$ and $\mathrm{AA}$ in the vasculature. As we know, it is very difficult to test the actual concentrations of aldehydes in the blood and in the cells since the aldehydes are so active and metabolism of aldehydes occurs quickly. One study showed the concentration of FA was in the range of $1.27 \times 10^{-2}$ to $2.28 \mu \mathrm{g} / \mathrm{mL}$ in the blood (Y. S. Wang et al., 2011), which looked much lower than the $\mathrm{EC}_{50}$ of relaxation in our study. The sensitivity of FA- and AA-induced relaxation (but not efficacy) was agonistdependent $\left(\mathrm{PE}<\mathrm{U} 46,619=\right.$ High $\left.\mathrm{K}^{+}\right)$(see Chapter VI, Table 22) and indicated $\mathrm{K}^{+}$ channel plays an important role in the relaxation of FA and AA. What's more, we show that Kir channel regulate the relaxation of FA.

\section{Aldehydes and TRPA1 Receptor}

Our study show several distinct pathways of FA-, AA-, and CR-induced vasorelaxation in SMA (see figures in Chapter III, IV, V). We focus on the mechanism of the most sensitive, and thus, most likely, physiological pathway. FA-, AA-, and CR-induced vasorelaxation requires a functional endothelium, NOS (presumably eNOS), and guanylyl cyclase (GC/cGMP), and what's more, FA- and CR- induced vasorelaxation in a similar pathway via the TRPA1 channel. It is likely that the TRPA1 and NOS components are in endothelial cells, whereas the latter is also present in vascular smooth muscle cells (VSMC). 
In addition, several unsaturated aldehydes including acrolein, cinnamaldehyde, and 4HNE induce sensitive and robust vasodilation in mesenteric arteries (Awe et al., 2006; Romero et al., 1997) and other blood vessels (Yanaga et al., 2006) as well as tracheal smooth muscle (Cheah et al., 2014), perhaps indicating a shared (universal) aldehyde-TRPA1 pathway. TRPA1 is a cation (calcium) channel and a promiscuous receptor concentrated in sensory fibers (unmyelinated C-fibers) that mediate pain reception/transmission (nociceptors), and it is also present in the vasculature (Earley et al., 2009a; Sinharoy et al., 2017). It is a well-known target of unsaturated aldehydes, such as acrolein and crotonaldehyde (Andre et al., 2008; Bessac et al., 2008; D. J. Conklin, 2016) and cinnamaldehyde (Pozsgai et al., 2010), but its activation by FA is somewhat surprising given the proposed mechanism of unsaturated aldehyde-mediated activation via conjugation with TRPA1 $\mathrm{N}$-terminal free cysteines (Macpherson et al., 2007). Activation of peripheral sensory fiber TRPA1 leads to pain signaling and release of vasoactive peptides, substance $P$ (SubP), and cGRP (Trevisan et al., 2016), which may contribute to increased vasodilation. We confirmed localization of TRPA1 in mouse dorsal root ganglion (DRG) and showed Immunofluorescent staining in the endothelium of murine SMA but not in aorta.

In our in vivo study, compared to the augmentation vasodilation in WT mice after CR exposure, there was no difference in the sensitivity or relaxation of ACh and SNP in CR group and air group in TRPA1-null mice after CR exposure; the compensatory changes in the vasculature were missing in TRPA1-null mice 
(see Chapter VI, Table 19,20 and Fig. 35). This disappearance of augmented

smooth muscle relaxation in the vasculature indicates that TRPA1 plays a specific role in the toxicity of CR.

TRPA1 is found in the pulmonary airways, and it plays a role in the protective physiological maneuver called "respiratory braking" elicited by inhaled toxins. Our previous study showed that TRPA1-null mice are more sensitive than WT mice to acrolein exposure because of its "respiratory braking", and that TRPA1 antagonist injection post-acrolein exposure reduces acrolein-induced mortality (D. J Conklin et al., 2017; D. J. Conklin et al., 2017). Our results show that TRPA1 receptor plays a much more important role in the vascular toxicity of the unsaturated aldehydes than saturated aldehydes. Future studies are needed to make clear whether TRPA1 plays a protective function or induces impairment to the blood vessels.

In our in vitro study, SMA is the main artery in the GI system, and food is a main source of endogenous aldehydes. Cho et al. showed that TRPA1 is present in sensory nerve endings and in some gastrointestinal enteroendocrine cells in the mouse gastrointestinal tract (Cho, Callaghan, Bron, Bravo, \& Furness, 2014). Our study also showed the immunofluorescence staining of TRPA1 present in the endothelium of the blood vessels in SMA. The specific TRPA1 antagonist, A967079 $\left(\mathrm{IC}_{50}=289 \mathrm{nM}\right)$, significantly blocked the most sensitive component of the FA- and $\mathrm{CR}$-induced relaxation and shifted the $\mathrm{EC}_{50}$ to the right quantitatively like that of endothelium disruption but less so than L-NAME treatment (see 
Chapter III, Fig. 11; Chapter V, Fig. 25). At high concentrations of FA and CR-likely at unphysiological levels of FA and CR—the TRPA1 antagonist did not affect relaxation, reflecting a non-TRPA1 pathway(s), perhaps via TRPV1 or TRPV4 channels (Ma et al., 2008). We cannot rule out the possibility that additional components in the vascular wall (perivascular nerves, SubP, CGRP, etc.) also may contribute to overall FA- and CR-induced relaxation (Nilius et al., 2012).

\section{Aldehydes and Nicotine}

Nicotine is an important component of tobacco smoke, and extensive research has shown nicotine is a main reason for increased cardiovascular events via activation of the sympathetic nervous system, increased myocardial remodeling, impaired lipid metabolism, and induction of inflammation (Benowitz \& Burbank, 2016; Dutta et al., 2012) . However, some papers show that nicotine is a double-edged sword which promotes angiogenesis and arteriogenesis on the contrary (Heeschen, Weis, \& Cooke, 2003; J. Lee \& Cooke, 2012).

Few studies show the interaction between nicotine and aldehydes in vivo, especially the effect of nicotine on the cardiovascular effects of aldehydes.

Nicotine could metabolize to methylamine and then metabolize to FA by semicarbazide-sensitive amine oxidase (SSAO) (D. J. Conklin et al., 2004; Yu, 1998). Previous studies have shown a dose-related interaction between nicotine 
and $\mathrm{AA}$ in conditioned taste-aversion (CTA) paradigm (Kunin, Latendresse, Gaskin, Smith, \& Amit, 2000).

One purpose in our study is to investigate the effect of nicotine on the function of aldehydes. The plateau concentration of nicotine in the blood

averaging $40 \mu \mathrm{g} / \mathrm{L}$ throughout the day in smokers (Moyer et al., 2002). In this study, we aim to mimic the vascular effect of nicotine and aldehydes when smoking or smoking as well as drinking. Our study showed that low concentrations of nicotine as well as $1 \mu \mathrm{M}$ does not affect the relaxation of FA, AA, or CR in SMA (see Chapter III, Chapter IV, Table 11,12; Chapter V, Fig. 29). Nicotine does not affect the vascular function of aldehydes in vitro.

Nicotine mainly binds and activates nicotine acetylcholine receptor (nAChR), but it is also a sensory agonist that activates TRPA1 and is responsible for the airway constriction reflex (Talavera et al., 2009). TRPA1 is a well-known target of acrolein, cinnamaldehyde, FA, and other aldehydes (Andre et al., 2008; Bessac et al., 2008; D. J. Conklin, 2016; Pozsgai et al., 2010). However, the function of TRPA1 in nicotine exposure and the effect of nicotine on aldehydes in vivo are still unclear.

\section{Aldehydes and sex difference}

This data in our study was consistent with our previous study, in which female mice exposed to a high level (210-250 ppm) of acrolein showed both 
pulmonary and systemic protection compared to their male counterparts, demonstrating reduced morbidity and a significantly lower sensitivity to acroleininduced mortality female mice showed a protective effect under acrolein exposure (D. J Conklin et al., 2017). Although both acute and chronic exposure to $C R$ induced vascular changes in male mice, females showed no changes in aortic sensitivity or reactivity in response to CR exposure in vivo (see Chapter VI, Fig. 36). These differences between males and females post-CR exposure seem to support our previous findings that females are somewhat protected against $\alpha, \beta$-unsaturated aldehyde-induced toxicity and injury. This is consistent with the human studies, rodent studies have also found that females are less sensitive than males in tobacco-induced atherosclerotic damage in part because of protective effects of female hormones (Bolego, Poli, \& Paoletti, 2002). Females had greater pain in some inflammatory conditions than males, which is under the control of prolactin (Patil, Green, Henry, \& Akopian, 2013). Prolactin receptor regulates transient receptor potential (TRP) channels in neurons including TRPA1, TRPV1, and TRPM8 and has a sex difference (Patil, Ruparel, Henry, \& Akopian, 2013). The mechanism of female-dependent protection in mice is not clear. Future studies are needed to explore the sex-dependent differences in respiratory, cardiovascular, and systemic toxicity in electronic cigarette aerosols or aldehyde exposures.

\section{Normalization of aorta contractility in testing vascular function}


Vascular function measurements are widely used in the study of pathogenesis of cardiovascular, cerebrovascular, pulmonary vascular, and kidney diseases (D. J. Conklin, 2016; D. J. Conklin et al., 2004; Earley, Gonzales, \& Crnich, 2009b; Jin, Jagatheesan, et al., 2019; Jin et al., 2018). In our in vitro studies, isolated vascular rings were hung on the organ bath systems and were in the Krebs-Henseleit equilibrium solution bubbling with a mixture of gases $\left(95 \% \mathrm{O}_{2}+5 \% \mathrm{CO}_{2}\right)$ to mimic physiological conditions. The blood vessels were given a certain preload by pulling by two wires to mimic a physiological or pathological condition in vivo. We then studied the contractility and relaxation of the blood vessels. Our method proved to be a reliable method for the comparison of contractility outcomes from different studies. We developed a simple 2-step aorta normalization method: (1) measure blood vessel segment length $(\mathrm{mm})$, area (mm2) and calculate volume (mm3); then, (2) normalize isometric contraction $(\mathrm{mN})$ by segment length and volume. We recommend that aortic contractions be normalized to segment length and/or volume to reduce variability, enhance efficiency, and to foster universal comparisons across isometric myograph platforms, laboratories, and experimental settings.

\section{B. Strength}

First, we show that the vascular toxicity of aldehydes in vivo exposure, which are absolutely associated with cigarette smoking. Second, this is the first study to show the vascular effects of CR exposure in vivo exposure, although the effect of endothelium dysfunction is limited. We also test the vasodilation of CR in vitro and showed the possible mechanism. Third, FA and AA are all very 
sensitive to the vasodilation of aorta and SMA. However, FA and AA at our exposure levels showed limited toxicity to blood vessels in vivo exposure or the direct effect to the blood vessels in vitro even at very high concentrations. Fourth, we tested the direct vascular effects of three aldehydes from low concentrations to high concentrations in vitro, which help us study the real biological changes to aldehydes exposure in life. Fifth, we show a good pharmacological model of endothelial dysfunction which is caused by air perfusion. Sixth, since the $85 \%$ vasorelaxation of SMA is EDHF dependent, it is a good choice to select SMA as a representative to study the function of small arteries, and it is the most important artery to control the blood flow to Gl system. Seventh, we show the function of TRPA1 receptor in the mediation of cardiovascular toxicity of CR. And, we show TRPA1 staining in the endothelium. TRPA1 might be the target for medical treatment after aldehydes exposure.

\section{Limitations}

There are important caveats to consider in this study. First, mice are obligate nose breathers and are "involuntary smokers," meaning that they decrease breathing rate and depth to reduce the inhalation of toxins such as acrolein (D. J Conklin et al., 2017). This reflex is known as the "respiratory braking response" (Alarie, 1973). The nose is very sensitive to aldehydes exposure, it would be swelling because of edema, and the mice even die of hypoxia because nose congestion. Second, low levels of FA, AA, and CR did not induce endothelial dysfunction, even in the chronic exposure of CR. This could 
be because the level of exposure was too low and/or because and the length of exposure was not sufficient to see significant changes. It is difficult to decide the exposure time for these inhalation experiment as several factors can complicate our desired outcomes. As we know, aldehydes are carcinogenic as well as irritants, and it is possible that cancer could develop before the development of negative cardiovascular effects during exposure. Third, we only tested the vascular function changes in aorta and SMA; we have not tested functional changes in other arteries such as the pulmonary artery and the third or fourth segments of mesenteric artery that might more sensitive to inhalation exposure. Fourth, we did not test whether TRPA1 antagonist had protective yet nonspecific effects, especially in the chronic exposure of CR. Testing the function of TRPA1 antagonist is needed in order to further explore the function of TRPA1 receptor. Fifth, perivascular adipose tissue (PVAT) is very important to the function of blood vessels, and there is a lot of TRPA1 receptor staining in the PVAT. We have not yet tested the effect of TRPA1 on the PVAT on the relaxation of blood vessels. Sixth, in vivo exposure study, the concentrations of aldehydes are continuous though the real smoking is intermittent since it's hard to prudence intermittent pattern during aldehyde exposure in our exposure system. Seventh, aldehydes are very active and the metabolisms of aldehydes are very fast. It's difficult to measure the concentration of aldehyde in the blood.

\section{Future direction}


Additional studies are still needed to further explore the toxicity of ecigarettes and cigarettes. We still have not identified the actual constituent that is responsible for the toxicity of smoking or how to cure the toxicity of smoking. We could test other possible mechanisms that mediate cardiovascular toxicity of aldehydes. In the future, we could increase the dose of aldehydes or the length of exposure to test whether it induces endothelial dysfunction. We could also test the vascular function changes of other arteries such as the pulmonary artery and smaller arteries such as the third or fourth segments of mesenteric artery. Additional studies could also be performed to determine the effect of TRPA1 antagonists during aldehydes exposure in mice as well as the effect of PVAT on the relaxation of blood vessels, including testing the function of TRPA1 receptor in PVAT. We could test CR exposure in atherosclerosis models to test whether TRPA1 could block atherosclerosis. We could test CR exposure in TRPA1-null mice. Finally, more studies are still needed to examine the effect of e-cigarette flavors on cardiovascular function such as testing blood pressure, heart rate, and body temperature changes during flavor exposures in mice.

\section{E. Conclusion}

These studies indicate that short-term exposures of mice to FA or AA have limited direct toxicity in aorta. However, CR exposures (acute and chronic) lead to altered vascular function that may lead to cardiovascular disease. Moreover, we describe direct effects of FA, AA, and CR that are dependent on an EDRF/NO and VSMC GC/PKG pathway in SMA, with FA- and CR-induced 
relaxations also dependent on an endothelium-localized TRPA1 receptor. From our in vitro study, we conclude that aldehydes play a key role in the regulation of blood flow in the gastrointestinal (Gl) tract to augment digestion and nutrient absorption and induce postprandial hyperemia, what's more, the TRPA1 receptor plays an important role in the aldehyde-induced vasodilation. Future studies are required to better understand how CR exposure modifies cardiovascular pathophysiology and the specific roles that TRPA1 and sex play in contributing to the burden of tobacco-related CVD including pathophysiological processes of atherosclerosis. 


\section{REFERENCES}

Abreu-Villaca, Y., Manhaes, A. C., Krahe, T. E., Filgueiras, C. C., \& Ribeiro-Carvalho, A. (2017). Tobacco and alcohol use during adolescence: Interactive mechanisms in animal models. Biochem Pharmacol, 144, 1-17. doi:10.1016/j.bcp.2017.06.113

Achanta, S., Chintagari, N. R., Brackmann, M., Balakrishna, S., \& Jordt, S. E. (2018). TRPA1 and CGRP antagonists counteract vesicant-induced skin injury and inflammation. Toxicol Lett, 293, 140-148. doi:10.1016/j.toxlet.2018.03.007

Administration., U. S. F. D. (2012). Harmful and potentially harmful constituents in tobacco products and tobacco smoke: Established list. . www.fda.gov.

Alarie, Y. (1973). Sensory irritation of the upper airways by airborne chemicals. Toxicol Appl Pharmacol, 24(2), 279-297.

Aldehydes, C. o. (1981). Formaldehyde and Other Aldehydes: National Academy Press, Washington D.C (1981).

Altura, B. M., \& Altura, B. T. (1982). Microvascular and vascular smooth muscle actions of ethanol, acetaldehyde, and acetate. Fed Proc, 41(8), 2447-2451. Retrieved from https://www.ncbi.nlm.nih.gov/pubmed/7044829

Altura, B. M., \& Altura, B. T. (1987). Peripheral and cerebrovascular actions of ethanol, acetaldehyde, and acetate: relationship to divalent cations. Alcohol Clin Exp Res, 11(2), 99-111. Retrieved from https://www.ncbi.nlm.nih.gov/pubmed/3296839

Altura, B. M., Altura, B. T., \& Carella, A. (1983). Ethanol produces coronary vasospasm: evidence for a direct action of ethanol on vascular muscle. Br J Pharmacol, 78(2), 260-262. doi:10.1111/j.1476-5381.1983.tb09389.x

Alwis, K. U., deCastro, B. R., Morrow, J. C., \& Blount, B. C. (2015). Acrolein Exposure in U.S. Tobacco Smokers and Non-Tobacco Users: NHANES 2005-2006. Environ Health Perspect, 123(12), 1302-1308. doi:10.1289/ehp.1409251

Ambrose, J. A., \& Barua, R. S. (2004). The pathophysiology of cigarette smoking and cardiovascular disease: an update. J Am Coll Cardiol, 43(10), 1731-1737. doi:10.1016/j.jacc.2003.12.047

Anand, U., Otto, W. R., Facer, P., Zebda, N., Selmer, I., Gunthorpe, M. J., . . . Anand, P. (2008). TRPA1 receptor localisation in the human peripheral nervous system and functional studies in cultured human and rat sensory neurons. Neurosci Lett, 438(2), 221-227. doi:10.1016/j.neulet.2008.04.007

Anderson, C., Majeste, A., Hanus, J., \& Wang, S. (2016). E-Cigarette Aerosol Exposure Induces Reactive Oxygen Species, DNA Damage, and Cell Death in Vascular Endothelial Cells. Toxicol Sci, 154(2), 332-340. doi:10.1093/toxsci/kfw166

Andrade, E. L., Meotti, F. C., \& Calixto, J. B. (2012). TRPA1 antagonists as potential analgesic drugs. Pharmacol Ther, 133(2), 189-204. doi:10.1016/j.pharmthera.2011.10.008

Andre, E., Campi, B., Materazzi, S., Trevisani, M., Amadesi, S., Massi, D., . . Patacchini, R. (2008). Cigarette smoke-induced neurogenic inflammation is mediated by 
alpha,beta-unsaturated aldehydes and the TRPA1 receptor in rodents. J Clin Invest, 118(7), 2574-2582. doi:10.1172/jci34886

Andrei, S. R., Sinharoy, P., Bratz, I. N., \& Damron, D. S. (2016). TRPA1 is functionally co-expressed with TRPV1 in cardiac muscle: Co-localization at z-discs, costameres and intercalated discs. Channels (Austin), 10(5), 395-409. doi:10.1080/19336950.2016.1185579

Asahara, T., Masuda, H., Takahashi, T., Kalka, C., Pastore, C., Silver, M., . . . Isner, J. M. (1999). Bone marrow origin of endothelial progenitor cells responsible for postnatal vasculogenesis in physiological and pathological neovascularization. Circ Res, 85(3), 221-228. doi:10.1161/01.res.85.3.221

Association., A. H. (2014). Smoking \& cardiovascular disease (heart disease). . www.heart.org.

Awe, S. O., Adeagbo, A. S., D'Souza, S. E., Bhatnagar, A., \& Conklin, D. J. (2006). Acrolein induces vasodilatation of rodent mesenteric bed via an EDHF-dependent mechanism. Toxicology and Applied Pharmacology, 217(3), 266-276. Retrieved from PM:17069868

Bandell, M., Story, G. M., Hwang, S. W., Viswanath, V., Eid, S. R., Petrus, M. J., . . . Patapoutian, A. (2004). Noxious cold ion channel TRPA1 is activated by pungent compounds and bradykinin. Neuron, 41(6), 849-857.

Barua, R. S., Ambrose, J. A., Srivastava, S., DeVoe, M. C., \& Eales-Reynolds, L. J. (2003). Reactive oxygen species are involved in smoking-induced dysfunction of nitric oxide biosynthesis and upregulation of endothelial nitric oxide synthase: an in vitro demonstration in human coronary artery endothelial cells. Circulation, 107(18), 2342-2347. doi:10.1161/01.cir.0000066691.52789.be

Bautista, D. M., Jordt, S. E., Nikai, T., Tsuruda, P. R., Read, A. J., Poblete, J., . . Julius, D. (2006). TRPA1 mediates the inflammatory actions of environmental irritants and proalgesic agents. Cell, 124(6), 1269-1282. doi:10.1016/j.cell.2006.02.023

Benjamin, E. J., Muntner, P., Alonso, A., Bittencourt, M. S., Callaway, C. W., Carson, A. P., . . Virani, S. S. (2019). Heart Disease and Stroke Statistics-2019 Update: A Report From the American Heart Association. Circulation, 139(10), e56-e528. doi:10.1161/cir.0000000000000659

Benowitz, N. L., \& Burbank, A. D. (2016). Cardiovascular toxicity of nicotine: Implications for electronic cigarette use. Trends Cardiovasc Med, 26(6), 515-523. doi:10.1016/j.tcm.2016.03.001

Benowitz, N. L., \& Fraiman, J. B. (2017). Cardiovascular effects of electronic cigarettes. Nat Rev Cardiol, 14(8), 447-456. doi:10.1038/nrcardio.2017.36

Benowitz, N. L., \& Gourlay, S. G. (1997). Cardiovascular toxicity of nicotine: implications for nicotine replacement therapy. J Am Coll Cardiol, 29(7), 1422-1431. doi:10.1016/s0735-1097(97)00079-x

Benowitz, N. L., Hansson, A., \& Jacob, P., 3rd. (2002). Cardiovascular effects of nasal and transdermal nicotine and cigarette smoking. Hypertension, 39(6), 1107-1112. doi:10.1161/01.hyp.0000018825.76673.ea

Beretta, M., Wolkart, G., Schernthaner, M., Griesberger, M., Neubauer, R., Schmidt, K., . . . Mayer, B. (2012). Vascular bioactivation of nitroglycerin is catalyzed by cytosolic aldehyde dehydrogenase-2. Circ Res, 110(3), 385-393. doi:10.1161/CIRCRESAHA.111.245837 
Berhane, K., Widersten, M., Engstrom, A., Kozarich, J. W., \& Mannervik, B. (1994). Detoxication of base propenals and other alpha, beta-unsaturated aldehyde products of radical reactions and lipid peroxidation by human glutathione transferases. Proc.Natl.Acad.Sci.U.S.A, 91(4), 1480-1484. Retrieved from PM:8108434

Bessac, B. F., Sivula, M., von Hehn, C. A., Escalera, J., Cohn, L., \& Jordt, S. E. (2008). TRPA1 is a major oxidant sensor in murine airway sensory neurons. $J$ Clin Invest, 118(5), 1899-1910. doi:10.1172/JCI34192

Bhatnagar, A. (2004). Cardiovascular pathophysiology of environmental pollutants. Am J Physiol Heart Circ Physiol, 286(2), H479-485. doi:10.1152/ajpheart.00817.2003

Bhatnagar, A. (2006). Environmental cardiology: studying mechanistic links between pollution and heart disease. Circ Res, 99(7), 692-705. doi:10.1161/01.RES.0000243586.99701.cf

Bhatnagar, A., Whitsel, L. P., Blaha, M. J., Huffman, M. D., Krishan-Sarin, S., Maa, J., . . . Warner, J. J. (2019). New and Emerging Tobacco Products and the Nicotine Endgame: The Role of Robust Regulation and Comprehensive Tobacco Control and Prevention: A Presidential Advisory From the American Heart Association. Circulation, 139(19), e937-e958. doi:10.1161/cir.0000000000000669

Bodkin, J. V., \& Brain, S. D. (2011). Transient receptor potential ankyrin 1: emerging pharmacology and indications for cardiovascular biology. Acta Physiol (Oxf), 203(1), 87-98. doi:10.1111/j.1748-1716.2010.02203.x

Bolego, C., Poli, A., \& Paoletti, R. (2002). Smoking and gender. Cardiovasc Res, 53(3), 568-576. doi:10.1016/s0008-6363(01)00520-X

Boor, P. J., \& Conklin, D. J. (2008). The arterial media as a target of structural and functional injury by chemicals. In D. Acosta (Ed.), Cardiovascular Toxicology (Fourth ed., pp. 667-691). New York: Informa Healthcare.

Carmichael, F. J., Israel, Y., Saldivia, V., Giles, H. G., Meggiorini, S., \& Orrego, H. (1987). Blood acetaldehyde and the ethanol-induced increase in splanchnic circulation. Biochem Pharmacol, 36(16), 2673-2678. doi:10.1016/0006-2952(87)90550-8

Carnevale, R., Sciarretta, S., Violi, F., Nocella, C., Loffredo, L., Perri, L., . . . Frati, G. (2016). Acute Impact of Tobacco vs Electronic Cigarette Smoking on Oxidative Stress and Vascular Function. Chest, 150(3), 606-612. doi:10.1016/j.chest.2016.04.012

Centers for Disease Control and Prevention. (2017). Smoking \& Tobacco Use.

Centers for Disease Control and Prevention. (2018). Smoking \& Tobacco Use. https://www.cdc.gov/tobacco/data_statistics/fact_sheets/health_effects/tobacco_re lated_mortality/index.htm

Chappell, D. C., Varner, S. E., Nerem, R. M., Medford, R. M., \& Alexander, R. W. (1998). Oscillatory shear stress stimulates adhesion molecule expression in cultured human endothelium. Circ Res, 82(5), 532-539. doi:10.1161/01.res.82.5.532

Cheah, E. Y., Burcham, P. C., Mann, T. S., \& Henry, P. J. (2014). Acrolein relaxes mouse isolated tracheal smooth muscle via a TRPA1-dependent mechanism. Biochem Pharmacol, 89(1), 148-156. doi:10.1016/j.bcp.2014.02.009

Chen, C. H., Budas, G. R., Churchill, E. N., Disatnik, M. H., Hurley, T. D., \& MochlyRosen, D. (2008). Activation of aldehyde dehydrogenase-2 reduces ischemic damage to the heart. Science, 321(5895), 1493-1495. doi:321/5895/1493 [pii]

10.1126/science. 1158554 
Chen, C. H., Ferreira, J. C., Gross, E. R., \& Mochly-Rosen, D. (2014). Targeting aldehyde dehydrogenase 2: new therapeutic opportunities. Physiol Rev, 94(1), 1-34. doi:10.1152/physrev.00017.2013

Cheng, C., de Crom, R., van Haperen, R., Helderman, F., Mousavi Gourabi, B., van Damme, L. C., . . Krams, R. (2004). The role of shear stress in atherosclerosis: action through gene expression and inflammation? Cell Biochem Biophys, 41(2), 279-294.

Cheng, C., Tempel, D., van Haperen, R., van der Baan, A., Grosveld, F., Daemen, M. J., . . . de Crom, R. (2006). Atherosclerotic lesion size and vulnerability are determined by patterns of fluid shear stress. Circulation, 113(23), 2744-2753. doi:10.1161/CIRCULATIONAHA.105.590018

Cheng, T. (2014). Chemical evaluation of electronic cigarettes. Tob Control, 23 Suppl 2, ii11-17. doi:10.1136/tobaccocontrol-2013-051482

Cho, H. J., Callaghan, B., Bron, R., Bravo, D. M., \& Furness, J. B. (2014). Identification of enteroendocrine cells that express TRPA1 channels in the mouse intestine. Cell Tissue Res, 356(1), 77-82. doi:10.1007/s00441-013-1780-x

Chou, C. C., \& Coatney, R. W. (1994). Nutrient-induced changes in intestinal blood flow in the dog. Br Vet J, 150(5), 423-437. doi:10.1016/S0007-1935(05)80192-7

Chung, F. L., Chen, H. J., \& Nath, R. G. (1996). Lipid peroxidation as a potential endogenous source for the formation of exocyclic DNA adducts. Carcinogenesis, 17(10), 2105-2111. doi:10.1093/carcin/17.10.2105

Chung, F. L., Nath, R. G., Nagao, M., Nishikawa, A., Zhou, G. D., \& Randerath, K. (1999). Endogenous formation and significance of 1,N2-propanodeoxyguanosine adducts. Mutat Res, 424(1-2), 71-81. doi:10.1016/s0027-5107(99)00009-3

Committee on, A. (1981). Formaldehyde and other aldehydes. Washington D.C. : National Academy Press.

Conklin, D. J. (2016). Acute cardiopulmonary toxicity of inhaled aldehydes: role of TRPA1. Ann N Y Acad Sci, 1374(1), 59-67. doi:10.1111/nyas.13055

Conklin, D. J., Barski, O. A., Lesgards, J. F., Juvan, P., Rezen, T., Rozman, D., . . . Bhatnagar, A. (2010). Acrolein consumption induces systemic dyslipidemia and lipoprotein modification. Toxicol Appl Pharmacol, 243(1), 1-12. doi:10.1016/j.taap.2009.12.010

Conklin, D. J., Bhatnagar, A., Cowley, H. R., Johnson, G. H., Wiechmann, R. J., Sayre, L. M., . . . Boor, P. J. (2006). Acrolein generation stimulates hypercontraction in isolated human blood vessels. Toxicology and Applied Pharmacology, 217(3), 277288. Retrieved from PM:17095030

Conklin, D. J., \& Boor, P. J. (1998). Allylamine cardiovascular toxicity: evidence for aberrant vasoreactivity in rats. Toxicol Appl Pharmacol, 148(2), 245-251. doi:10.1006/taap.1997.8331

Conklin, D. J., Boyce, C. L., Trent, M. B., \& Boor, P. J. (2001a). Amine metabolism: a novel path to coronary artery vasospasm. Toxicology and Applied Pharmacology, 175(2), 149-159. Retrieved from PM:11543647

Conklin, D. J., Boyce, C. L., Trent, M. B., \& Boor, P. J. (2001b). Amine metabolism: a novel path to coronary artery vasospasm. Toxicol Appl Pharmacol, 175(2), 149159. doi:10.1006/taap.2001.9238 
Conklin, D. J., Cowley, H. R., Wiechmann, R. J., Johnson, G. H., Trent, M. B., \& Boor, P. J. (2004). Vasoactive effects of methylamine in isolated human blood vessels: role of semicarbazide-sensitive amine oxidase, formaldehyde, and hydrogen peroxide. Am J Physiol Heart Circ Physiol, 286(2), H667-676. doi:10.1152/ajpheart.00690.2003

Conklin, D. J., Guo, Y., Jagatheesan, G., Nystoriak, M. A., Obal, D., Kilfoil, P., . . . Bhatnagar, A. (2019). Transient Receptor Potential Ankyrin-1 (TRPA1) Channel Contributes to Myocardial Ischemia-Reperfusion Injury. Am J Physiol Heart Circ Physiol. doi:10.1152/ajpheart.00106.2018

Conklin, D. J., Guo, Y., Nystoriak, M. A., Jagatheesan, G., Obal, D., Kilfoil, P. J., . . . Bhatnagar, A. (2019). TRPA1 channel contributes to myocardial ischemiareperfusion injury. American Journal of Physiology-Heart and Circulatory Physiology, 316(4), H889-H899. doi:10.1152/ajpheart.00106.2018

Conklin, D. J., Haberzettl, P., Jagatheesan, G., Baba, S., Merchant, M. L., Prough, R. A., . . Bhatnagar, A. (2015a). Glutathione $S$-transferase $P$ protects against cyclophosphamide-induced cardiotoxicity in mice. Toxicol Appl Pharmacol, 285(2), 136-148. doi:10.1016/j.taap.2015.03.029

Conklin, D. J., Haberzettl, P., Jagatheesan, G., Baba, S., Merchant, M. L., Prough, R. A., , . Bhatnagar, A. (2015b). Glutathione S-transferase $\mathrm{P}$ protects against cyclophosphamide-induced cardiotoxicity in mice. Toxicol Appl Pharmacol, 285(2), 136-148. doi:10.1016/j.taap.2015.03.029

Conklin, D. J., Haberzettl, P., Jagatheesan, G., Kong, M., \& Hoyle, G. W. (2016). Role of TRPA1 in acute cardiopulmonary toxicity of inhaled acrolein. Toxicol Appl Pharmacol. doi:10.1016/j.taap.2016.08.028

Conklin, D. J., Haberzettl, P., Jagatheesan, G., Kong, M., \& Hoyle, G. W. (2017). Role of TRPA1 in acute cardiopulmonary toxicity of inhaled acrolein. Toxicol Appl Pharmacol, 324, 61-72. doi:10.1016/j.taap.2016.08.028

Conklin, D. J., Haberzettl, P., Lee, J., \& Srivastava, S. (2011). Aldehydes and Cardiovascular Disease. London: Royal Society of Chemistry: A. Bhatnagar.

Conklin, D. J., Haberzettl, P., Lee, J., \& Srivastava, S. (2011). Aldehydes and Cardiovascular Disease. In A. Bhatnagar (Ed.), Environmental Cardiology: Pollution and Heart Disease (Vol. 8, pp. 390). London, England: Royal Society of Chemistry.

Conklin, D. J., Haberzettl, P., Lesgards, J. F., Prough, R. A., Srivastava, S., \& Bhatnagar, A. (2009). Increased sensitivity of glutathione S-transferase P-null mice to cyclophosphamide-induced urinary bladder toxicity. J Pharmacol Exp Ther, 331(2), 456-469. doi:10.1124/jpet.109.156513

Conklin, D. J., Haberzettl, P., Prough, R. A., \& Bhatnagar, A. (2009a). Glutathione-Stransferase $\mathrm{P}$ protects against endothelial dysfunction induced by exposure to tobacco smoke. Am.J.Physiol Heart Circ.Physiol, 296(5), H1586-H1597. Retrieved from PM:19270193

Conklin, D. J., Haberzettl, P., Prough, R. A., \& Bhatnagar, A. (2009b). Glutathione-Stransferase $\mathrm{P}$ protects against endothelial dysfunction induced by exposure to tobacco smoke. Am J Physiol Heart Circ Physiol, 296(5), H1586-1597. doi:10.1152/ajpheart.00867.2008 
Conklin, D. J., Kong, M., \& Committee, H. E. I. H. R. (2015). Part 4. Assessment of plasma markers and cardiovascular responses in rats after chronic exposure to newtechnology diesel exhaust in the ACES bioassay. Res Rep Health Eff Inst(184), 111139; discussion 141-171. Retrieved from http://www.ncbi.nlm.nih.gov/pubmed/25842618

Conklin, D. J., Langford, S. D., \& Boor, P. J. (1998). Contribution of serum and cellular semicarbazide-sensitive amine oxidase to amine metabolism and cardiovascular toxicity. Toxicol.Sci., 46(2), 386-392. Retrieved from PM:10048142

Conklin, D. J., Malovichko, M. V., Zeller, I., Das, T. P., Krivokhizhina, T. V., Lynch, B. H., . . Srivastava, S. (2017). Biomarkers of Chronic Acrolein Inhalation Exposure in Mice: Implications for Tobacco Product-Induced Toxicity. Toxicol Sci, 158(2), 263-274. doi:10.1093/toxsci/kfx095

Conklin, D. J., Malovichko, M. V., Zeller, I., Das, T. P., Krivokhizhina, T. V., Lynch, B. H., ... Srivastava, S. (2017). Biomarkers of Chronic Acrolein Inhalation Exposure in Mice: Implications for Tobacco Product-Induced Toxicity. Toxicological Sciences, 158(2), 263-274. doi:10.1093/toxsci/kfx095

Conklin, D. J., Ogunwale, M. A., Chen, Y., Theis, W. S., Nantz, M. H., Fu, X. A., . . . Srivastava, S. (2018). Electronic cigarette-generated aldehydes: The contribution of e-liquid components to their formation and the use of urinary aldehyde metabolites as biomarkers of exposure. Aerosol Sci Technol, 52(11), 1219-1232. doi:10.1080/02786826.2018.1500013

Conklin, D. J., Prough, R. A., Juvan, P., Rezen, T., Rozman, D., Haberzettl, P., . . . Bhatnagar, A. (2011). Acrolein-induced dyslipidemia and acute-phase response are independent of HMG-CoA reductase. Mol Nutr Food Res, 55(9), 1411-1422. doi:10.1002/mnfr.201100225

Conklin, D. J., Schick, S., Blaha, M. J., Carll, A., DeFilippis, A., Ganz, P., . . Bhatnagar, A. (2019). Cardiovascular injury induced by tobacco products: assessment of risk factors and biomarkers of harm. A Tobacco Centers of Regulatory Science compilation. Am J Physiol Heart Circ Physiol, 316(4), H801-H827. doi:10.1152/ajpheart.00591.2018

Cullen, K. A., Ambrose, B. K., Gentzke, A. S., Apelberg, B. J., Jamal, A., \& King, B. A. (2018). Notes from the Field: Use of Electronic Cigarettes and Any Tobacco Product Among Middle and High School Students - United States, 2011-2018. MMWR Morb Mortal Wkly Rep, 67(45), 1276-1277. doi:10.15585/mmwr.mm6745a5

Daiber, A., Steven, S., Weber, A., Shuvaev, V. V., Muzykantov, V. R., Laher, I., . . . Munzel, T. (2017). Targeting vascular (endothelial) dysfunction. Br J Pharmacol, 174(12), 1591-1619. doi:10.1111/bph.13517

Deanfield, J. E., Halcox, J. P., \& Rabelink, T. J. (2007). Endothelial function and dysfunction: testing and clinical relevance. Circulation, 115(10), 1285-1295. doi:10.1161/circulationaha.106.652859

Deng, Y., \& Yu, P. H. (1999). Simultaneous determination of formaldehyde and methylglyoxal in urine: involvement of semicarbazide-sensitive amine oxidasemediated deamination in diabetic complications. J.Chromatogr.Sci., 37(9), 317322. 
Duescher, R. J., \& Elfarra, A. A. (1993). Chloroperoxidase-mediated oxidation of 1,3butadiene to 3-butenal, a crotonaldehyde precursor. Chem Res Toxicol, 6(5), 669673. doi:10.1021/tx00035a012

Duschek, S., Hoffmann, A., \& Reyes Del Paso, G. A. (2017). Affective impairment in chronic low blood pressure. J Psychosom Res, 93, 33-40. doi:10.1016/j.jpsychores.2016.12.008

Dutta, P., Courties, G., Wei, Y., Leuschner, F., Gorbatov, R., Robbins, C. S., . . . Nahrendorf, M. (2012). Myocardial infarction accelerates atherosclerosis. Nature, 487(7407), 325-329. doi:10.1038/nature 11260

Earley, S. (2012). TRPA1 channels in the vasculature. Br J Pharmacol, 167(1), 13-22. doi:10.1111/j.1476-5381.2012.02018.x

Earley, S., Gonzales, A. L., \& Crnich, R. (2009a). Endothelium-dependent cerebral artery dilation mediated by TRPA1 and Ca2+-Activated K+ channels. Circ Res, 104(8), 987-994. doi:10.1161/circresaha.108.189530

Earley, S., Gonzales, A. L., \& Crnich, R. (2009b). Endothelium-Dependent Cerebral Artery Dilation Mediated by TRPA1 and Ca2+-Activated K+ Channels. Circulation Research. Retrieved from PM:19299646

Eder, E., Schuler, D., \& Budiawan. (1999). Cancer risk assessment for crotonaldehyde and 2-hexenal: an approach. IARC Sci Publ(150), 219-232.

Egle, J. L., Jr., Hudgins, P. M., \& Lai, F. M. (1973). Cardiovascular effects of intravenous acetaldehyde and propionaldehyde in the anesthetized rat. Toxicol Appl Pharmacol, 24(4), 636-644. Retrieved from https://www.ncbi.nlm.nih.gov/pubmed/4713486

Egleton, R. D., Brown, K. C., \& Dasgupta, P. (2009). Angiogenic activity of nicotinic acetylcholine receptors: implications in tobacco-related vascular diseases. Pharmacol Ther, 121(2), 205-223. doi:10.1016/j.pharmthera.2008.10.007

Espinoza-Derout, J., Hasan, K. M., Shao, X. M., Jordan, M. C., Sims, C., Lee, D. L., . . . Friedman, T. C. (2019). Chronic intermittent electronic cigarette exposure induces cardiac dysfunction and atherosclerosis in apolipoprotein-E knockout mice. Am J Physiol Heart Circ Physiol, 317(2), H445-h459. doi:10.1152/ajpheart.00738.2018

Facchinetti, F., Amadei, F., Geppetti, P., Tarantini, F., Di Serio, C., Dragotto, A., . . . Patacchini, R. (2007). Alpha,beta-unsaturated aldehydes in cigarette smoke release inflammatory mediators from human macrophages. Am J Respir Cell Mol Biol, 37(5), 617-623. doi:10.1165/rcmb.2007-0130OC

Filser, J. G., Faller, T. H., Bhowmik, S., Schuster, A., Kessler, W., Putz, C., \& Csanady, G. A. (2001). First-pass metabolism of 1,3-butadiene in once-through perfused livers of rats and mice. Chem Biol Interact, 135-136, 249-265.

Fukuto, J. M., Gulati, P., \& Nagasawa, H. T. (1994). Involvement of nitroxyl (HNO) in the cyanamide-induced vasorelaxation of rabbit aorta. Biochem Pharmacol, 47(5), 922-924. doi:10.1016/0006-2952(94)90495-2

Furchgott, R. F., \& Zawadzki, J. V. (1980). The obligatory role of endothelial cells in the relaxation of arterial smooth muscle by acetylcholine. Nature, 288(5789), 373-376. doi:10.1038/288373a0

Gentzke, A. S., Creamer, M., Cullen, K. A., Ambrose, B. K., Willis, G., Jamal, A., \& King, B. A. (2019). Vital Signs: Tobacco Product Use Among Middle and High School Students - United States, 2011-2018. MMWR Morb Mortal Wkly Rep, 68(6), 157164. doi:10.15585/mmwr.mm6806e1 
Goniewicz, M. L., Gawron, M., Smith, D. M., Peng, M., Jacob, P., 3rd, \& Benowitz, N. L. (2017). Exposure to Nicotine and Selected Toxicants in Cigarette Smokers Who Switched to Electronic Cigarettes: A Longitudinal Within-Subjects Observational Study. Nicotine Tob Res, 19(2), 160-167. doi:10.1093/ntr/ntw160

Gray, J. M., \& Barnsley, E. A. (1971). The metabolism of crotyl phosphate, crotyl alcohol and crotonaldehyde. Xenobiotica, 1(1), 55-67. Retrieved from http://www.ncbi.nlm.nih.gov/entrez/query.fcgi?cmd=Retrieve\&db=PubMed\&dop $\mathrm{t}=$ Citation\&list_uids $=4356091$

Green, M. A., \& Egle, J. L., Jr. (1983). The effects of acetaldehyde and acrolein on blood pressure in guanethidine-pretreated hypertensive rats. Toxicology and Applied Pharmacology, 69(1), 29-36. Retrieved from PM:6857685

Green, N., Weech, M., \& Walters, E. (2005). Localization and characterization of glutathione-s-transferase isozymes alpha, mu, and pi within the mouse vomeronasal organ. Neurosci.Lett., 375(3), 198-202. Retrieved from PM:15694260

Greenberg, S. S., Xie, J., Wang, Y., Kolls, J., Shellito, J., Nelson, S., \& Summer, W. R. (1993). Ethanol relaxes pulmonary artery by release of prostaglandin and nitric oxide. Alcohol, 10(1), 21-29. Retrieved from https://www.ncbi.nlm.nih.gov/pubmed/8447963

Gross, E. R., Zambelli, V. O., Small, B. A., Ferreira, J. C., Chen, C. H., \& Mochly-Rosen, D. (2015). A personalized medicine approach for Asian Americans with the aldehyde dehydrogenase 2*2 variant. Annu Rev Pharmacol Toxicol, 55, 107-127. doi:10.1146/annurev-pharmtox-010814-124915

Hahn, J., Monakhova, Y. B., Hengen, J., Kohl-Himmelseher, M., Schussler, J., Hahn, H., . . . Lachenmeier, D. W. (2014). Electronic cigarettes: overview of chemical composition and exposure estimation. Tob Induc Dis, 12(1), 23. doi:10.1186/s12971-014-0023-6

Haussmann, H. J. (2012). Use of hazard indices for a theoretical evaluation of cigarette smoke composition. Chem Res Toxicol, 25(4), 794-810. doi:10.1021/tx200536w

Hecht, S. S., Upadhyaya, P., \& Wang, M. (1999). Reactions of alpha-acetoxy-Nnitrosopyrrolidine and crotonaldehyde with DNA. IARC Sci Publ(150), 147-154.

Heeschen, C., Weis, M., \& Cooke, J. P. (2003). Nicotine promotes arteriogenesis. J Am Coll Cardiol, 41(3), 489-496. doi:10.1016/s0735-1097(02)02818-8

Heidenreich, P. A., Trogdon, J. G., Khavjou, O. A., Butler, J., Dracup, K., Ezekowitz, M. D., ... Outcomes, R. (2011). Forecasting the future of cardiovascular disease in the United States: a policy statement from the American Heart Association. Circulation, 123(8), 933-944. doi:10.1161/CIR.0b013e31820a55f5

Hill, B. G., Haberzettl, P., Ahmed, Y., Srivastava, S., \& Bhatnagar, A. (2008). Unsaturated lipid peroxidation-derived aldehydes activate autophagy in vascular smoothmuscle cells. Biochem J, 410(3), 525-534. doi:10.1042/BJ20071063

Hill, G. E., Miller, J. A., Baxter, B. T., Klassen, L. W., Duryee, M. J., Tuma, D. J., \& Thiele, G. M. (1998). Association of malondialdehyde-acetaldehyde (MAA) adducted proteins with atherosclerotic-induced vascular inflammatory injury. Atherosclerosis, 141(1), 107-116.

Himmelstein, M. W., Acquavella, J. F., Recio, L., Medinsky, M. A., \& Bond, J. A. (1997). Toxicology and epidemiology of 1,3-butadiene. Crit Rev Toxicol, 27(1), 1-108. doi: $10.3109 / 10408449709037482$ 
Hink, U., Daiber, A., Kayhan, N., Trischler, J., Kraatz, C., Oelze, M., . . Munzel, T. (2007). Oxidative inhibition of the mitochondrial aldehyde dehydrogenase promotes nitroglycerin tolerance in human blood vessels. J Am Coll Cardiol, 50(23), 22262232. doi:10.1016/j.jacc.2007.08.031

Hobara, N., Watanabe, A., Kobayashi, M., Nakatsukasa, H., Nagashima, H., Fukuda, T., \& Araki, Y. (1985). Tissue distribution of acetaldehyde in rats following acetaldehyde inhalation and intragastric ethanol administration. Bull Environ Contam Toxicol, 35(3), 393-396. doi:10.1007/bf01636528

Hom, S., Chen, L., Wang, T., Ghebrehiwet, B., Yin, W., \& Rubenstein, D. A. (2016). Platelet activation, adhesion, inflammation, and aggregation potential are altered in the presence of electronic cigarette extracts of variable nicotine concentrations. Platelets, 27(7), 694-702. doi:10.3109/09537104.2016.1158403

Hughson, W. G., Mann, J. I., \& Garrod, A. (1978). Intermittent claudication: prevalence and risk factors. $\mathrm{Br} \mathrm{Med} J, 1(6124), 1379-1381$. doi:10.1136/bmj.1.6124.1379

Hutzler, C., Paschke, M., Kruschinski, S., Henkler, F., Hahn, J., \& Luch, A. (2014). Chemical hazards present in liquids and vapors of electronic cigarettes. Arch Toxicol, 88(7), 1295-1308. doi:10.1007/s00204-014-1294-7

Huveneers, S., Daemen, M. J., \& Hordijk, P. L. (2015). Between Rho(k) and a hard place: the relation between vessel wall stiffness, endothelial contractility, and cardiovascular disease. Circ Res, 116(5), 895-908. doi:10.1161/circresaha.116.305720

IARC. (1995). Crotonaldehyde.: International Agency of Research on Cancer.

Inoue, R., Jensen, L. J., Shi, J., Morita, H., Nishida, M., Honda, A., \& Ito, Y. (2006). Transient receptor potential channels in cardiovascular function and disease. Circ Res, 99(2), 119-131. doi:10.1161/01.RES.0000233356.10630.8a

Jain, R. B. (2015). Distributions of selected urinary metabolites of volatile organic compounds by age, gender, race/ethnicity, and smoking status in a representative sample of U.S. adults. Environ Toxicol Pharmacol, 40(2), 471-479. doi:10.1016/j.etap.2015.07.018

Jamal, M., Ameno, K., Tanaka, N., Ito, A., Takakura, A., Kumihashi, M., \& Kinoshita, H. (2016). Ethanol and Acetaldehyde After Intraperitoneal Administration to Aldh2Knockout Mice-Reflection in Blood and Brain Levels. Neurochem Res, 41(5), 1029-1034. doi:10.1007/s11064-015-1788-6

Jiang, M., Wan, F., Wang, F., \& Wu, Q. (2015). Irisin relaxes mouse mesenteric arteries through endothelium-dependent and endothelium-independent mechanisms. Biochem Biophys Res Commun, 468(4), 832-836. doi:10.1016/j.bbrc.2015.11.040

Jin, L., Jagatheesan, G., Guo, L., Nystoriak, M., Malovichko, M., Lorkiewicz, P., . . . Conklin, D. J. (2019). Formaldehyde Induces Mesenteric Artery Relaxation via a Sensitive Transient Receptor Potential Ankyrin-1 (TRPA1) and EndotheliumDependent Mechanism: Potential Role in Postprandial Hyperemia. Front Physiol, 10, 277. doi:10.3389/fphys.2019.00277

Jin, L., Lipinski, A., \& Conklin, D. J. (2018). A Simple Method for Normalization of Aortic Contractility. J Vasc Res, 55(3), 177-186. doi:10.1159/000490245

Jin, L., Lorkiewicz, P., Malovichko, M. V., Bhatnagar, A., Srivastava, S., \& Conklin, D. J. (2019). Acetaldehyde Induces an Endothelium-Dependent Relaxation of Superior 
Mesenteric Artery: Potential Role in Postprandial Hyperemia. Front Physiol, 10, 1315. doi:10.3389/fphys.2019.01315

Kage, S., Kudo, K., Ikeda, H., \& Ikeda, N. (2004). Simultaneous determination of formate and acetate in whole blood and urine from humans using gas chromatography-mass spectrometry. J Chromatogr B Analyt Technol Biomed Life Sci, 805(1), 113-117. doi:10.1016/j.jchromb.2004.02.029

Kaiser, R., Holmquist, B., Vallee, B. L., \& Jornvall, H. (1991). Human class III alcohol dehydrogenase/glutathione-dependent formaldehyde dehydrogenase. J Protein Chem, 10(1), 69-73. Retrieved from http://www.ncbi.nlm.nih.gov/pubmed/2054065

Kawaguchi-Niida, M., Shibata, N., Morikawa, S., Uchida, K., Yamamoto, T., Sawada, T., \& Kobayashi, M. (2006). Crotonaldehyde accumulates in glial cells of Alzheimer's disease brain. Acta Neuropathol, 111(5), 422-429. doi:10.1007/s00401-006-00441

Kim, S. K., Avila, J. J., \& Massett, M. P. (2016). Strain survey and genetic analysis of vasoreactivity in mouse aorta. Physiol Genomics, physiolgenomics 0005402016. doi:10.1152/physiolgenomics.00054.2016

Kleinnijenhuis, A. J., Staal, Y. C., Duistermaat, E., Engel, R., \& Woutersen, R. A. (2013). The determination of exogenous formaldehyde in blood of rats during and after inhalation exposure. Food Chem Toxicol, 52, 105-112. doi:10.1016/j.fct.2012.11.008

Kolluru, G. K., Bir, S. C., \& Kevil, C. G. (2012). Endothelial dysfunction and diabetes: effects on angiogenesis, vascular remodeling, and wound healing. Int J Vasc Med, 2012, 918267. doi:10.1155/2012/918267

Kunin, D., Latendresse, M. W., Gaskin, S., Smith, B. R., \& Amit, Z. (2000). Preexposure effects of nicotine and acetaldehyde on conditioned taste aversion induced by both drugs. Pharmacol Biochem Behav, 66(4), 695-699. doi:10.1016/s00913057(00)00284-7

Kurhanewicz, N., Ledbetter, A., Farraj, A., \& Hazari, M. (2018). TRPA1 mediates the cardiac effects of acrolein through parasympathetic dominance but also sympathetic modulation in mice. Toxicol Appl Pharmacol, 347, 104-114. doi:10.1016/j.taap.2018.03.027

Kurhanewicz, N., McIntosh-Kastrinsky, R., Tong, H., Ledbetter, A., Walsh, L., Farraj, A., \& Hazari, M. (2017). TRPA1 mediates changes in heart rate variability and cardiac mechanical function in mice exposed to acrolein. Toxicol Appl Pharmacol, 324, 5160. doi:10.1016/j.taap.2016.10.008

Lamarre, S. G., MacMillan, L., Morrow, G. P., Randell, E., Pongnopparat, T., Brosnan, M. E., \& Brosnan, J. T. (2014). An isotope-dilution, GC-MS assay for formate and its application to human and animal metabolism. Amino Acids, 46(8), 1885-1891. doi:10.1007/s00726-014-1738-7

Lee, B. P., Morton, R. F., \& Lee, L. Y. (1992). Acute effects of acrolein on breathing: role of vagal bronchopulmonary afferents. J Appl Physiol (1985), 72(3), 1050-1056. doi:10.1152/jappl.1992.72.3.1050

Lee, J., \& Cooke, J. P. (2012). Nicotine and pathological angiogenesis. Life Sci, 91(21-22), 1058-1064. doi:10.1016/j.lfs.2012.06.032 
Lee, S. E., \& Park, Y. S. (2013). Role of lipid peroxidation-derived alpha, beta-unsaturated aldehydes in vascular dysfunction. Oxid Med Cell Longev, 2013, 629028. doi:10.1155/2013/629028

Lerman, A., \& Zeiher, A. M. (2005). Endothelial function: cardiac events. Circulation, 111(3), 363-368. doi:10.1161/01.cir.0000153339.27064.14

Levy D, W. P. (2000). Atherosclerotic cardiovascular disease: an epidemiological perspective. In T. E (Ed.), Comprehensive Cardiovascular Medicine (Vol. 1998, pp. 27-43). Philadelphia: Lippincott-Raven.

Li, P., Zhu, N., Yi, B., Wang, N., Chen, M., You, X., . . Sun, J. (2013). MicroRNA-663 regulates human vascular smooth muscle cell phenotypic switch and vascular neointimal formation. Circ Res, 113(10), 1117-1127. doi:10.1161/circresaha.113.301306

Liao, S., Luo, C., Cao, B., Hu, H., Wang, S., Yue, H., . . Zhou, Z. (2017). Endothelial Progenitor Cells for Ischemic Stroke: Update on Basic Research and Application. Stem Cells Int, 2017, 2193432. doi:10.1155/2017/2193432

Lindros, K. O., Stowell, A., Pikkarainen, P., \& Salaspuro, M. (1980). Elevated blood acetaldehyde in alcoholics with accelerated ethanol elimination. Pharmacol Biochem Behav, 13 Suppl 1, 119-124. Retrieved from https://www.ncbi.nlm.nih.gov/pubmed/7243824

Lino-dos-Santos-Franco, A., Amemiya, R. M., Ligeiro de Oliveira, A. P., BreithauptFaloppa, A. C., Damazo, A. S., Oliveira-Filho, R. M., \& Tavares-de-Lima, W. (2011). Differential effects of female sex hormones on cellular recruitment and tracheal reactivity after formaldehyde exposure. Toxicol Lett, 205(3), 327-335. doi:10.1016/j.toxlet.2011.06.023

Lino-dos-Santos-Franco, A., Domingos, H. V., de Oliveira, A. P., Breithaupt-Faloppa, A. C., Peron, J. P., Bolonheis, S., . . Tavares-de-Lima, W. (2010). Differential effects of formaldehyde exposure on the cell influx and vascular permeability in a rat model of allergic lung inflammation. Toxicol Lett, 197(3), 211-218.

Liu, X. Y., Yang, Z. H., Pan, X. J., Zhu, M. X., \& Xie, J. P. (2010a). Crotonaldehyde induces oxidative stress and caspase-dependent apoptosis in human bronchial epithelial cells. Toxicol Lett, 195(1), 90-98. doi:10.1016/j.toxlet.2010.02.004

Liu, X. Y., Yang, Z. H., Pan, X. J., Zhu, M. X., \& Xie, J. P. (2010b). Gene expression profile and cytotoxicity of human bronchial epithelial cells exposed to crotonaldehyde. Toxicol Lett, 197(2), 113-122. doi:10.1016/j.toxlet.2010.05.005

Lorkiewicz, P., Riggs, D. W., Keith, R. J., Conklin, D. J., Xie, Z., Sutaria, S., .. Bhatnagar, A. (2019). Comparison of Urinary Biomarkers of Exposure in Humans Using Electronic Cigarettes, Combustible Cigarettes, and Smokeless Tobacco. Nicotine Tob Res, 21(9), 1228-1238. doi:10.1093/ntr/nty089

Lucchini, S., Saumet, J. L., Mei, N., \& Garnier, L. (1996). Involvement of the vagus nerve, substance $\mathrm{P}$ and cholecystokinin in the regulation of intestinal blood flow. J Auton Nerv Syst, 60(3), 182-192. Retrieved from https://www.ncbi.nlm.nih.gov/pubmed/8912269

Luscher, T. F. (1990). Imbalance of endothelium-derived relaxing and contracting factors. A new concept in hypertension? Am $J$ Hypertens, 3(4), 317-330. doi:10.1093/ajh/3.4.317 
Ma, Y. Y., Huo, H. R., Li, C. H., Zhao, B. S., Li, L. F., Sui, F., . . Jiang, T. L. (2008). Effects of cinnamaldehyde on PGE2 release and TRPV4 expression in mouse cerebral microvascular endothelial cells induced by interleukin-1beta. Biol.Pharm.Bull., 31(3), 426-430. Retrieved from PM:18310904

Macpherson, L. J., Dubin, A. E., Evans, M. J., Marr, F., Schultz, P. G., Cravatt, B. F., \& Patapoutian, A. (2007). Noxious compounds activate TRPA1 ion channels through covalent modification of cysteines. Nature, 445(7127), 541-545. doi:10.1038/nature05544

Makia, N. L., Bojang, P., Falkner, K. C., Conklin, D. J., \& Prough, R. A. (2011). Murine hepatic aldehyde dehydrogenase $1 \mathrm{a} 1$ is a major contributor to oxidation of aldehydes formed by lipid peroxidation. Chem Biol Interact, 191(1-3), 278-287. doi:10.1016/j.cbi.2011.01.013

Malek, A. M., Alper, S. L., \& Izumo, S. (1999). Hemodynamic shear stress and its role in atherosclerosis. JAMA, 282(21), 2035-2042. doi:10.1001/jama.282.21.2035

Martin, E. M., Clapp, P. W., Rebuli, M. E., Pawlak, E. A., Glista-Baker, E., Benowitz, N. L., . . . Jaspers, I. (2016). E-cigarette use results in suppression of immune and inflammatory-response genes in nasal epithelial cells similar to cigarette smoke. Am J Physiol Lung Cell Mol Physiol, 311(1), L135-144. doi:10.1152/ajplung.00170.2016

Martinez, M. C., Bosch-Morell, F., Raya, A., Roma, J., Aldasoro, M., Vila, J., . . Romero, F. J. (1994). 4-Hydroxynonenal, a lipid peroxidation product, induces relaxation of human cerebral arteries. J.Cereb.Blood Flow Metab, 14(4), 693-696. Retrieved from PM:8014218

Matanoski, G. M., Santos-Burgoa, C., \& Schwartz, L. (1990). Mortality of a cohort of workers in the styrene-butadiene polymer manufacturing industry (1943-1982). Environ Health Perspect, 86, 107-117. doi:10.1289/ehp.9086107

Materazzi, S., Fusi, C., Benemei, S., Pedretti, P., Patacchini, R., Nilius, B., . . Nassini, R. (2012). TRPA1 and TRPV4 mediate paclitaxel-induced peripheral neuropathy in mice via a glutathione-sensitive mechanism. Pflugers Arch, 463(4), 561-569. doi:10.1007/s00424-011-1071-x

McNamara, C. R., Mandel-Brehm, J., Bautista, D. M., Siemens, J., Deranian, K. L., Zhao, M., . . Fanger, C. M. (2007). TRPA1 mediates formalin-induced pain. Proc Natl Acad Sci U S A, 104(33), 13525-13530. doi:10.1073/pnas.0705924104

Messner, B., \& Bernhard, D. (2014). Smoking and cardiovascular disease: mechanisms of endothelial dysfunction and early atherogenesis. Arterioscler Thromb Vasc Biol, 34(3), 509-515. doi:10.1161/atvbaha.113.300156

Moheimani, R. S., Bhetraratana, M., Yin, F., Peters, K. M., Gornbein, J., Araujo, J. A., \& Middlekauff, H. R. (2017). Increased Cardiac Sympathetic Activity and Oxidative Stress in Habitual Electronic Cigarette Users: Implications for Cardiovascular Risk. JAMA Cardiol, 2(3), 278-284. doi:10.1001/jamacardio.2016.5303

Moyer, T. P., Charlson, J. R., Enger, R. J., Dale, L. C., Ebbert, J. O., Schroeder, D. R., \& Hurt, R. D. (2002). Simultaneous analysis of nicotine, nicotine metabolites, and tobacco alkaloids in serum or urine by tandem mass spectrometry, with clinically relevant metabolic profiles. Clin Chem, 48(9), 1460-1471. 
Murphy, R. A. (1980). Mechanics of Vascular Smooth Muscle. In Comprehensive Physiology (Vol. Supplement 7: Handbook of Physiology, The Cardiovascular System, Vascular Smooth Muscle, pp. 325-351).

Nagata, K., Duggan, A., Kumar, G., \& Garcia-Anoveros, J. (2005). Nociceptor and hair cell transducer properties of TRPA1, a channel for pain and hearing. J Neurosci, 25(16), 4052-4061. doi:10.1523/jneurosci.0013-05.2005

Nair, U., Bartsch, H., \& Nair, J. (2007). Lipid peroxidation-induced DNA damage in cancer-prone inflammatory diseases: a review of published adduct types and levels in humans. Free Radic Biol Med, 43(8), 1109-1120. doi:10.1016/j.freeradbiomed.2007.07.012

Nauhaus, S. K., Fennell, T. R., Asgharian, B., Bond, J. A., \& Sumner, S. C. (1996). Characterization of urinary metabolites from Sprague-Dawley rats and B6C3F1 mice exposed to [1,2,3,4-13C]butadiene. Chem Res Toxicol, 9(4), 764-773. doi:10.1021/tx950196u

Nehler, M. R., Duval, S., Diao, L., Annex, B. H., Hiatt, W. R., Rogers, K., . . Hirsch, A. T. (2014). Epidemiology of peripheral arterial disease and critical limb ischemia in an insured national population. J Vasc Surg, 60(3), 686-695.e682. doi:10.1016/j.jvs.2014.03.290

Nilius, B., Appendino, G., \& Owsianik, G. (2012). The transient receptor potential channel TRPA1: from gene to pathophysiology. Pflugers Arch, 464(5), 425-458. doi:10.1007/s00424-012-1158-z

O'Toole, T. E., Abplanalp, W., Li, X., Cooper, N., Conklin, D. J., Haberzettl, P., \& Bhatnagar, A. (2014). Acrolein decreases endothelial cell migration and insulin sensitivity through induction of let-7a. Toxicol Sci, 140(2), 271-282. doi:10.1093/toxsci/kfu087

Ogunwale, M. A., Li, M., Ramakrishnam Raju, M. V., Chen, Y., Nantz, M. H., Conklin, D. J., \& Fu, X. A. (2017). Aldehyde Detection in Electronic Cigarette Aerosols. ACS Omega, 2(3), 1207-1214. doi:10.1021/acsomega.6b00489

Olfert, I. M., DeVallance, E., Hoskinson, H., Branyan, K. W., Clayton, S., Pitzer, C. R., .. . Chantler, P. D. (2018). Chronic exposure to electronic cigarettes results in impaired cardiovascular function in mice. J Appl Physiol (1985), 124(3), 573-582. doi:10.1152/japplphysiol.00713.2017

Organization., W. H. (2017). Who report on the global tobacco epidemic, 2017: Monitoring tobacco use and prevention policies. .

Owens, P., \& O'Brien, E. (1999). Hypotension in patients with coronary disease: can profound hypotensive events cause myocardial ischaemic events? Heart, 82(4), 477-481. doi:10.1136/hrt.82.4.477

Palmer, R. M., Ferrige, A. G., \& Moncada, S. (1987). Nitric oxide release accounts for the biological activity of endothelium-derived relaxing factor. Nature, 327(6122), 524526. doi:10.1038/327524a0

Park, Y. S., \& Taniguchi, N. (2008). Acrolein induces inflammatory response underlying endothelial dysfunction: a risk factor for atherosclerosis. Ann N Y Acad Sci, 1126, 185-189. doi:10.1196/annals.1433.034

Patil, M. J., Green, D. P., Henry, M. A., \& Akopian, A. N. (2013). Sex-dependent roles of prolactin and prolactin receptor in postoperative pain and hyperalgesia in mice. Neuroscience, 253, 132-141. doi:10.1016/j.neuroscience.2013.08.035 
Patil, M. J., Ruparel, S. B., Henry, M. A., \& Akopian, A. N. (2013). Prolactin regulates TRPV1, TRPA1, and TRPM8 in sensory neurons in a sex-dependent manner: Contribution of prolactin receptor to inflammatory pain. Am J Physiol Endocrinol Metab, 305(9), E1154-1164. doi:10.1152/ajpendo.00187.2013

Pazo, D. Y., Moliere, F., Sampson, M. M., Reese, C. M., Agnew-Heard, K. A., Walters, M. J., ... Chambers, D. M. (2016). Mainstream Smoke Levels of Volatile Organic Compounds in 50 U.S. Domestic Cigarette Brands Smoked With the ISO and Canadian Intense Protocols. Nicotine Tob Res, 18(9), 1886-1894. doi:10.1093/ntr/ntw118

Pei, Z., Zhuang, Z., Sang, H., Wu, Z., Meng, R., He, E. Y., . . Ren, J. (2014). alpha,betaUnsaturated aldehyde crotonaldehyde triggers cardiomyocyte contractile dysfunction: role of TRPV1 and mitochondrial function. Pharmacol Res, 82, 4050. doi:10.1016/j.phrs.2014.03.010

Pelosi, E., Castelli, G., \& Testa, U. (2014). Endothelial progenitors. Blood Cells Mol Dis, 52(4), 186-194. doi:10.1016/j.bcmd.2013.11.004

Penn, A., \& Snyder, C. A. (1996a). 1,3 Butadiene, a vapor phase component of environmental tobacco smoke, accelerates arteriosclerotic plaque development. Circulation, 93(3), 552-557. doi:10.1161/01.cir.93.3.552

Penn, A., \& Snyder, C. A. (1996b). Butadiene inhalation accelerates arteriosclerotic plaque development in cockerels. Toxicology, 113(1-3), 351-354.

Polverino, M., Polverino, F., Fasolino, M., Ando, F., Alfieri, A., \& De Blasio, F. (2012). Anatomy and neuro-pathophysiology of the cough reflex arc. Multidiscip Respir Med, 7(1), 5. doi:10.1186/2049-6958-7-5

Powell, J. T., Edwards, R. J., Worrell, P. C., Franks, P. J., Greenhalgh, R. M., \& Poulter, N. R. (1997). Risk factors associated with the development of peripheral arterial disease in smokers: a case-control study. Atherosclerosis, 129(1), 41-48.

Pozsgai, G., Bodkin, J. V., Graepel, R., Bevan, S., Andersson, D. A., \& Brain, S. D. (2010). Evidence for the pathophysiological relevance of TRPA1 receptors in the cardiovascular system in vivo. Cardiovasc Res, 87(4), 760-768. doi: $10.1093 / \mathrm{cvr} / \mathrm{cvq} 118$

cvq118 [pii]

Prevention., C. f. D. C. a. (2017). Health effects of secondhand smoke. . www.cdc.gov.

Raij, L., DeMaster, E. G., \& Jaimes, E. A. (2001). Cigarette smoke-induced endothelium dysfunction: role of superoxide anion. J Hypertens, 19(5), 891-897.

Ramana, K. V., Bhatnagar, A., Srivastava, S., Yadav, U. C., Awasthi, S., Awasthi, Y. C., \& Srivastava, S. K. (2006). Mitogenic responses of vascular smooth muscle cells to lipid peroxidation-derived aldehyde 4-hydroxy-trans-2-nonenal (HNE): role of aldose reductase-catalyzed reduction of the HNE-glutathione conjugates in regulating cell growth. $J$ Biol Chem, 281(26), 17652-17660. doi:10.1074/jbc.M600270200

Reddy, S., Finkelstein, E. I., Wong, P. S., Phung, A., Cross, C. E., \& van der Vliet, A. (2002). Identification of glutathione modifications by cigarette smoke. Free Radic Biol Med, 33(11), 1490-1498. doi:S0891584902010791 [pii]

Ren, J., \& Brown, R. A. (2000). Influence of chronic alcohol ingestion on acetaldehydeinduced depression of rat cardiac contractile function. Alcohol Alcohol, 35(6), 554560. doi:10.1093/alcalc/35.6.554 
Reynolds, S., Williams, A. S., Williams, H., Smale, S., Stephenson, H. J., Amos, N., . . . Lang, D. (2012). Contractile, but not endothelial, dysfunction in early inflammatory arthritis: a possible role for matrix metalloproteinase-9. Br J Pharmacol, 167(3), 505-514. doi:10.1111/j.1476-5381.2012.01988.x

Richardson, K. A., Peters, M. M., Megens, R. H., van Elburg, P. A., Golding, B. T., Boogaard, P. J., . . . van Sittert, N. J. (1998). Identification of novel metabolites of butadiene monoepoxide in rats and mice. Chem Res Toxicol, 11(12), 1543-1555. doi:10.1021/tx970175v

Rocha, J. T., Hipolito, U. V., Callera, G. E., Yogi, A., Neto Filho Mdos, A., Bendhack, L. M., . . . Tirapelli, C. R. (2012). Ethanol induces vascular relaxation via redoxsensitive and nitric oxide-dependent pathways. Vascul Pharmacol, 56(1-2), 74-83. doi:10.1016/j.vph.2011.11.006

Romero, F. J., Romero, M. J., Bosch-Morell, F., Martinez, M. C., Medina, P., \& Lluch, S. (1997). 4-hydroxynonenal-induced relaxation of human mesenteric arteries. Free Radical Biology and Medicine, 23(3), 521-523. Retrieved from PM:9214591

Roth, G. A., Johnson, C. O., Abate, K. H., Abd-Allah, F., Ahmed, M., Alam, K., .. Murray, C. J. L. (2018). The Burden of Cardiovascular Diseases Among US States, 19902016. JAMA Cardiol, 3(5), 375-389. doi:10.1001/jamacardio.2018.0385

Ru, X. C., Qian, L. B., Gao, Q., Li, Y. F., Bruce, I. C., \& Xia, Q. (2008). Alcohol induces relaxation of rat thoracic aorta and mesenteric arterial bed. Alcohol Alcohol, 43(5), 537-543. doi:10.1093/alcalc/agn042

Russell, F. A., King, R., Smillie, S. J., Kodji, X., \& Brain, S. D. (2014). Calcitonin generelated peptide: physiology and pathophysiology. Physiol Rev, 94(4), 1099-1142. doi:10.1152/physrev.00034.2013

Salaspuro, M. (2017). Key role of local acetaldehyde in upper GI tract carcinogenesis. Best Pract Res Clin Gastroenterol, 31(5), 491-499. doi:10.1016/j.bpg.2017.09.016

Santanam, N., Thornhill, B. A., Lau, J. K., Crabtree, C. M., Cook, C. R., Brown, K. C., \& Dasgupta, P. (2012). Nicotinic acetylcholine receptor signaling in atherogenesis. Atherosclerosis, 225(2), 264-273. doi:10.1016/j.atherosclerosis.2012.07.041

Scarino, A., Tardif, R., \& Charbonneau, M. (2009). Influence of ALDH2 polymorphism on ethanol kinetics and pulmonary effects in male and female rats exposed to ethanol vapors. Inhal Toxicol, 21(3), 193-199. doi:904258751 [pii]

Schachinger, V., Britten, M. B., \& Zeiher, A. M. (2000). Prognostic impact of coronary vasodilator dysfunction on adverse long-term outcome of coronary heart disease. Circulation, 101(16), 1899-1906. doi:10.1161/01.cir.101.16.1899

Schepelmann, M., Yarova, P. L., Lopez-Fernandez, I., Davies, T. S., Brennan, S. C., Edwards, P. J., . . . Riccardi, D. (2016). The vascular Ca2+-sensing receptor regulates blood vessel tone and blood pressure. Am J Physiol Cell Physiol, 310(3), C193-204. doi:10.1152/ajpcell.00248.2015

Schwartz, E. S., Christianson, J. A., Chen, X., La, J. H., Davis, B. M., Albers, K. M., \& Gebhart, G. F. (2011). Synergistic role of TRPV1 and TRPA1 in pancreatic pain and inflammation. Gastroenterology, 140(4), 1283-1291 e1281-1282. doi:10.1053/j.gastro.2010.12.033

Shibasaki, K., Ogawa, S., Yamada, S., Ouchi, Y., \& Akishita, M. (2018). Role of autonomic nervous activity, as measured by heart rate variability, on the effect of 
mortality in disabled older adults with low blood pressure in long-term care. Geriatr Gerontol Int, 18(8), 1153-1158. doi:10.1111/ggi.13328

Silva, J. F., Correa, I. C., Diniz, T. F., Lima, P. M., Santos, R. L., Cortes, S. F., . . Lemos, V. S. (2016). Obesity, Inflammation, and Exercise Training: Relative Contribution of iNOS and eNOS in the Modulation of Vascular Function in the Mouse Aorta. Front Physiol, 7, 386. doi:10.3389/fphys.2016.00386

Simon, S. A., \& Liedtke, W. (2008). How irritating: the role of TRPA1 in sensing cigarette smoke and aerogenic oxidants in the airways. J Clin Invest, 118(7), 2383-2386. doi:10.1172/jci36111

Sinha, S., Sinharoy, P., Bratz, I. N., \& Damron, D. S. (2015). Propofol causes vasodilation in vivo via TRPA1 ion channels: role of nitric oxide and $\mathrm{BKCa}$ channels. PLoS One, 10(4), e0122189. doi:10.1371/journal.pone.0122189

Sinharoy, P., Bratz, I. N., Sinha, S., Showalter, L. E., Andrei, S. R., \& Damron, D. S. (2017). TRPA1 and TRPV1 contribute to propofol-mediated antagonism of U46619induced constriction in murine coronary arteries. PLoS One, 12(6), e0180106. doi:10.1371/journal.pone.0180106

Sithu, S. D., Malovichko, M. V., Riggs, K. A., Wickramasinghe, N. S., Winner, M. G., Agarwal, A., . . Srivastava, S. (2017). Atherogenesis and metabolic dysregulation in LDL receptor-knockout rats. JCI Insight, 2(9). doi:10.1172/jci.insight.86442

Sithu, S. D., Srivastava, S., Siddiqui, M. A., Vladykovskaya, E., Riggs, D. W., Conklin, D. J., . . . D'Souza, S. E. (2010). Exposure to acrolein by inhalation causes platelet activation. Toxicol Appl Pharmacol, 248(2), 100-110. doi:10.1016/j.taap.2010.07.013

Smith, C. J., \& Fischer, T. H. (2001). Particulate and vapor phase constituents of cigarette mainstream smoke and risk of myocardial infarction. Atherosclerosis, 158(2), 257267. doi:10.1016/s0021-9150(01)00570-6

Solleveld, H. A., Miller, R. A., Banas, D. A., \& Boorman, G. A. (1988). Primary cardiac hemangiosarcomas induced by 1,3-butadiene in B6C3F1 hybrid mice. Toxicol Pathol, 16(1), 46-52. doi:10.1177/019262338801600106

Srivastava, S., Conklin, D. J., Liu, S. Q., Prakash, N., Boor, P. J., Srivastava, S. K., \& Bhatnagar, A. (2001). Identification of biochemical pathways for the metabolism of oxidized low-density lipoprotein derived aldehyde-4-hydroxy trans-2-nonenal in vascular smooth muscle cells. Atherosclerosis, 158(2), 339-350. Retrieved from PM:11583712

Srivastava, S., Sithu, S. D., Vladykovskaya, E., Haberzettl, P., Hoetker, D. J., Siddiqui, M. A., . . . Bhatnagar, A. (2011). Oral exposure to acrolein exacerbates atherosclerosis in apoE-null mice. Atherosclerosis, 215(2), 301-308. doi:10.1016/j.atherosclerosis.2011.01.001

Srivastava, S., Vladykovskaya, E., Barski, O. A., Spite, M., Kaiserova, K., Petrash, J. M., . . . Bhatnagar, A. (2009). Aldose reductase protects against early atherosclerotic lesion formation in apolipoprotein E-null mice. Circ Res, 105(8), 793-802. doi:10.1161/CIRCRESAHA.109.200568

Stabbert, R., Dempsey, R., Diekmann, J., Euchenhofer, C., Hagemeister, T., Haussmann, H. J., . . . Veltel, D. J. (2017). Studies on the contributions of smoke constituents, individually and in mixtures, in a range of in vitro bioactivity assays. Toxicol In Vitro, 42, 222-246. doi:10.1016/j.tiv.2017.04.003 
Strubelt, O., Brasch, H., Pentz, R., \& Younes, M. (1990). Experimental studies on the acute cardiovascular toxicity of formalin and its antidotal treatment. J Toxicol Clin Toxicol, 28(2), 221-233. Retrieved from http://www.ncbi.nlm.nih.gov/pubmed/2168953

Takagi, T., Naruse, S., \& Shionoya, S. (1988). Postprandial celiac and superior mesenteric blood flows in conscious dogs. Am $J$ Physiol, $255(4$ Pt 1), G522-528. doi:10.1152/ajpgi.1988.255.4.G522

Talavera, K., Gees, M., Karashima, Y., Meseguer, V. M., Vanoirbeek, J. A., Damann, N., . . . Voets, T. (2009). Nicotine activates the chemosensory cation channel TRPA1. Nat Neurosci, 12(10), 1293-1299. doi:10.1038/nn.2379

Tan, Y., Li, X., Prabhu, S. D., Brittian, K. R., Chen, Q., Yin, X., . . . Cai, L. (2012). Angiotensin II plays a critical role in alcohol-induced cardiac nitrative damage, cell death, remodeling, and cardiomyopathy in a protein kinase $\mathrm{C} /$ nicotinamide adenine dinucleotide phosphate oxidase-dependent manner. J Am Coll Cardiol, 59(16), 1477-1486. doi:10.1016/j.jacc.2011.12.034

Tani, T. (1981). [Relaxation of vascular smooth muscle induced by formaldehyde (author's transl)]. Nihon Yakurigaku Zasshi, 77(2), 221-230. Retrieved from https://www.ncbi.nlm.nih.gov/pubmed/7239363

Tani, T., \& Horiguchi, Y. (1990). Effects of formaldehyde on cardiac function. Jpn J Pharmacol, 52(4), 563-572. Retrieved from https://www.ncbi.nlm.nih.gov/pubmed/2342227

Tani, T., Kogi, K., \& Horiguchi, Y. (1986). Inhibitory effects of formaldehyde inhalation on the cardiovascular and respiratory systems in unanesthetized rabbits. Jpn J Pharmacol, 40(4), 551-559. Retrieved from https://www.ncbi.nlm.nih.gov/pubmed/3735804

Tani, T., Satoh, S., \& Horiguchi, Y. (1978). The vasodilator action of formaldehyde in dogs. Toxicol Appl Pharmacol, 43(3), 493-499. Retrieved from https://www.ncbi.nlm.nih.gov/pubmed/418528

Toda, N., Konishi, M., Miyazaki, M., \& Komura, S. (1983). The effects of ethanol and acetaldehyde on dog arterial smooth muscle. J Stud Alcohol, 44(1), 1-16. Retrieved from https://www.ncbi.nlm.nih.gov/pubmed/6865419

Tomioka, H., Hattori, Y., Fukao, M., Sato, A., Liu, M., Sakuma, I., . . Kanno, M. (1999). Relaxation in different-sized rat blood vessels mediated by endothelium-derived hyperpolarizing factor: importance of processes mediating precontractions. $J$ Vasc Res, 36(4), 311-320. doi:10.1159/000025659

Trevisan, G., Benemei, S., Materazzi, S., De Logu, F., De Siena, G., Fusi, C., . . Nassini, R. (2016). TRPA1 mediates trigeminal neuropathic pain in mice downstream of monocytes/macrophages and oxidative stress. Brain, 139(Pt 5), 1361-1377. doi:10.1093/brain/aww038

Trevisani, M., Siemens, J., Materazzi, S., Bautista, D. M., Nassini, R., Campi, B., . . . Geppetti, P. (2007). 4-Hydroxynonenal, an endogenous aldehyde, causes pain and neurogenic inflammation through activation of the irritant receptor TRPA1. Proc Natl Acad Sci U S A, 104(33), 13519-13524. doi:10.1073/pnas.0705923104

Tsujimoto, T., \& Kajio, H. (2018). Low diastolic blood pressure and adverse outcomes in heart failure with preserved ejection fraction. Int $J$ Cardiol, 263, 69-74. doi:10.1016/j.ijcard.2018.04.031 
Tsukamoto, S., Kanegae, T., Saito, M., Nagoya, T., Shimamura, M., Tainaka, H., \& Kawaguchi, M. (1991). Concentrations of blood and urine ethanol, acetaldehyde, acetate and acetone during experimental hangover in volunteers. Arukoru Kenkyuto Yakubutsu Ison, 26(6), 500-510. Retrieved from http://www.ncbi.nlm.nih.gov/pubmed/1785958

Tuma, D. J., Hoffman, T., \& Sorrell, M. F. (1991). The chemistry of acetaldehyde-protein adducts. Alcohol Alcohol Suppl, 1, 271-276.

U.S. Department of Health and Human Services. (2014). The health consequences of smoking - 50 years of progress: a report of the Surgeon General.

Ueta, C. B., Campos, J. C., Albuquerque, R. P. E., Lima, V. M., Disatnik, M. H., Sanchez, A. B., . . Ferreira, J. C. B. (2018). Cardioprotection induced by a brief exposure to acetaldehyde: role of aldehyde dehydrogenase 2. Cardiovasc Res, 114(7), 10061015. doi:10.1093/cvr/cvy070

Vakevainen, S., Tillonen, J., Agarwal, D. P., Srivastava, N., \& Salaspuro, M. (2000). High salivary acetaldehyde after a moderate dose of alcohol in ALDH2-deficient subjects: strong evidence for the local carcinogenic action of acetaldehyde. Alcohol Clin Exp Res, 24(6), 873-877. Retrieved from https://www.ncbi.nlm.nih.gov/pubmed/10888077

van Andel I, S. E., Rambali B, Wolterink G, van de Werken G, Stevenson H, van Aerts LAGJM, Vleeming W. (2017). The health- and addictive effectes due to exposure to aldehydes of cigarette smoke., Part 1; acetaldehyde, formaldehyde, acrolein and propionaldehyde. .

Vanhoutte, P. M., Shimokawa, H., Feletou, M., \& Tang, E. H. (2017). Endothelial dysfunction and vascular disease - a 30th anniversary update. Acta Physiol (Oxf), 219(1), 22-96. doi:10.1111/apha.12646

Vasa, M., Fichtlscherer, S., Aicher, A., Adler, K., Urbich, C., Martin, H., . . Dimmeler, S. (2001). Number and migratory activity of circulating endothelial progenitor cells inversely correlate with risk factors for coronary artery disease. Circ Res, 89(1), E1-7. doi:10.1161/hh1301.093953

Vasiliou, V., \& Nebert, D. W. (2005). Analysis and update of the human aldehyde dehydrogenase (ALDH) gene family. Hum Genomics, 2(2), 138-143.

Versari, D., Daghini, E., Virdis, A., Ghiadoni, L., \& Taddei, S. (2009). Endothelial dysfunction as a target for prevention of cardiovascular disease. Diabetes Care, 32 Suppl 2, S314-321. doi:10.2337/dc09-S330

Vita, J. A., \& Keaney, J. F., Jr. (2002). Endothelial function: a barometer for cardiovascular risk? Circulation, 106(6), 640-642. doi:10.1161/01.cir.0000028581.07992.56

Vita, J. A., \& Loscalzo, J. (2002). Shouldering the risk factor burden: infection, atherosclerosis, and the vascular endothelium. Circulation, 106(2), 164-166. doi:10.1161/01.cir.0000023452.26135.34

Voulgaridou, G. P., Anestopoulos, I., Franco, R., Panayiotidis, M. I., \& Pappa, A. (2011). DNA damage induced by endogenous aldehydes: current state of knowledge. Mutat Res, 711(1-2), 13-27. doi:10.1016/j.mrfmmm.2011.03.006

Wang, M. Y., Chung, F. L., \& Hecht, S. S. (1988). Identification of crotonaldehyde as a hepatic microsomal metabolite formed by alpha-hydroxylation of the carcinogen N-nitrosopyrrolidine. Chem Res Toxicol, 1(1), 28-31. 
Wang, Y. S., Tan, X., Xue, J. H., Li, G. R., Shi, L. F., Yang, H. M., . . Xiao, X. L. (2011). Determination of trace formaldehyde in blood plasma by resonance fluorescence technology. Anal Chim Acta, 690(2), 234-239. doi:10.1016/j.aca.2011.02.030

Weind, K. L., Ellis, C. G., \& Boughner, D. R. (2002). Aortic valve cusp vessel density: relationship with tissue thickness. J Thorac Cardiovasc Surg, 123(2), 333-340.

Wetzelberger, K., Baba, S. P., Thirunavukkarasu, M., Ho, Y. S., Maulik, N., Barski, O. A., ... Bhatnagar, A. (2010). Postischemic deactivation of cardiac aldose reductase: role of glutathione S-transferase $\mathrm{P}$ and glutaredoxin in regeneration of reduced thiols from sulfenic acids. $J$ Biol Chem, 285(34), 26135-26148. doi:10.1074/jbc.M110.146423

Wheat, L. A., Haberzettl, P., Hellmann, J., Baba, S. P., Bertke, M., Lee, J., . . Conklin, D. J. (2011). Acrolein inhalation prevents vascular endothelial growth factor-induced mobilization of Flk-1+/Sca-1+ cells in mice. Arterioscler Thromb Vasc Biol, 31(7), 1598-1606. doi:10.1161/atvbaha.111.227124

World Health Organization. (2019). Tobacco. https://www.who.int/news-room/factsheets/detail/tobacco

Writing Group, M., Mozaffarian, D., Benjamin, E. J., Go, A. S., Arnett, D. K., Blaha, M. J., . . Stroke Statistics, S. (2019). Heart Disease and Stroke Statistics-2016 Update: A Report From the American Heart Association. Circulation, e481-483. doi:DOI: 10.1161/CIR.0000000000000659

Yanaga, A., Goto, H., Nakagawa, T., Hikiami, H., Shibahara, N., \& Shimada, Y. (2006). Cinnamaldehyde induces endothelium-dependent and -independent vasorelaxant action on isolated rat aorta. Biol.Pharm.Bull., 29(12), 2415-2418. Retrieved from PM: 17142974

Yang, B. C., Pan, X. J., Yang, Z. H., Xiao, F. J., Liu, X. Y., Zhu, M. X., \& Xie, J. P. (2013a). Crotonaldehyde induces apoptosis in alveolar macrophages through intracellular calcium, mitochondria and p53 signaling pathways. J Toxicol Sci, 38(2), 225-235. doi:10.2131/jts.38.225

Yang, B. C., Yang, Z. H., Pan, X. J., Liu, X. Y., Zhu, M. X., \& Xie, J. P. (2013b). Crotonaldehyde induces apoptosis and immunosuppression in alveolar macrophages. Toxicol In Vitro, 27(1), 128-137. doi:10.1016/j.tiv.2012.09.008

Yannoutsos, A., Levy, B. I., Safar, M. E., Slama, G., \& Blacher, J. (2014). Pathophysiology of hypertension: interactions between macro and microvascular alterations through endothelial dysfunction. $J$ Hypertens, 32(2), 216-224. doi:10.1097/hjh.0000000000000021

Yokoyama, A., Tsutsumi, E., Imazeki, H., Suwa, Y., Nakamura, C., Mizukami, T., \& Yokoyama, T. (2008). Salivary acetaldehyde concentration according to alcoholic beverage consumed and aldehyde dehydrogenase-2 genotype. Alcohol Clin Exp Res, 32(9), 1607-1614. doi:10.1111/j.1530-0277.2008.00739.x

$\mathrm{Yu}, \mathrm{P} . \mathrm{H}$. (1998). Increase of formation of methylamine and formaldehyde in vivo after administration of nicotine and the potential cytotoxicity. Neurochem Res, 23(9), 1205-1210. doi:10.1023/a:1020786219966

Yu, P. H., \& Deng, Y. L. (1998). Endogenous formaldehyde as a potential factor of vulnerability of atherosclerosis: involvement of semicarbazide-sensitive amine oxidase-mediated methylamine turnover. Atherosclerosis, 140(2), 357-363. Retrieved from http://www.ncbi.nlm.nih.gov/pubmed/9862279 
Yu, P. H., \& Zuo, D. M. (1996). Formaldehyde produced endogenously via deamination of methylamine. A potential risk factor for initiation of endothelial injury. Atherosclerosis, 120(1-2), 189-197. Retrieved from https://www.ncbi.nlm.nih.gov/pubmed/8645360

Zhang, Q., Tian, P., Zhai, M., Lei, X., Yang, Z., Liu, Y., . . . Meng, Z. (2018). Formaldehyde regulates vascular tensions through nitric oxide-cGMP signaling pathway and ion channels. Chemosphere, 193, 60-73. doi:10.1016/j.chemosphere.2017.11.013

Zhang, Z., Wang, M., Fan, X. H., Chen, J. H., Guan, Y. Y., \& Tang, Y. B. (2012). Upregulation of TRPM7 channels by angiotensin II triggers phenotypic switching of vascular smooth muscle cells of ascending aorta. Circ Res, 111(9), 1137-1146. doi:10.1161/circresaha.112.273755 


\section{ABBREVIATIONS}

$\begin{array}{ll}\text { AA } & \text { Acetaldehyde } \\ \text { ACh } & \text { Acetylcholine chloride } \\ \text { ADH } & \text { Alcohol dehydrogenase } \\ \text { AITC } & \text { Allyl isothiocyanate }\end{array}$

ALDH2 Acetaldehyde dehydrogenase 2

ALT Alanine transaminase

ANOVA Analysis variation

AR Aldose reductase

AST Aspartate transaminase

$\mathrm{BaCl}_{2} \quad$ Barium chloride

CACs Circulating angiogenic cells

CK creatinine kinase

CR Crotonaldehyde

CVD Cardiovascular disease

CO Cardiac output

EC Endothelial cell

$\mathrm{EC}_{50} \quad$ Half maximal effective concentration 


\begin{tabular}{|c|c|}
\hline EDHF & Endothelium-Derived Hyperpolarizing Factor \\
\hline EDRF & Endothelium-Derived Relaxing factor \\
\hline EPC & Endothelial progenitor cell \\
\hline FA & Formaldehyde \\
\hline FALDH2 & Formaldehyde dehydrogenase \\
\hline GC & Guanylyl cyclase \\
\hline GI & Gastrointestinal \\
\hline GSH & Glutathione \\
\hline GSTP & Glutathione S-transferase P \\
\hline GTT & Glucose tolerance test \\
\hline HbA1c & Whole blood hemoglobin A1c \\
\hline HPMMA & 3-hydroxy-1-methylpropylmercapturic acid \\
\hline 3-HPMA & 3-hydroxypropyl mercapturic acid \\
\hline 4HNE & and 4-hydroxynonenal \\
\hline HR & Heart rate \\
\hline IL-6 & Interleukin 6 \\
\hline IVRT & Isovolumic relaxation time \\
\hline LDH & Lactate dehydrogenase \\
\hline LDLR-KO & Low-density lipoprotein receptor deficient \\
\hline
\end{tabular}




\begin{tabular}{|c|c|}
\hline L-NAME & N\&-Nitro-Larginine methyl ester hydrochloride \\
\hline MCS & Mainstream cigarette smoke \\
\hline NO & Nitric oxide \\
\hline NOS & Nitric oxide synthase \\
\hline ODQ & $1 \mathrm{~h}$-[1,2,4]oxadiazolo[4,3-a]quinoxalin-1-one \\
\hline PE & L-phenylephrine hydrochloride \\
\hline PKG & Protein Kinase G \\
\hline PMAs & Platelet-monocyte aggregates \\
\hline PSS & Phosphate-buffered solution \\
\hline PWV & Pulse wave velocity \\
\hline SMA & Superior mesenteric artery \\
\hline SNP & Sodium nitroprusside \\
\hline TEA & Tetraethylammonium chloride \\
\hline TNFa & tumor necrosis factor alpha \\
\hline TRPA1 & Ttransient receptor potential ankyrin 1 \\
\hline VSMC & Vascular smooth muscle cells \\
\hline WT & Wild type \\
\hline
\end{tabular}




\author{
CURRICULUM VITAE \\ Lexiao Jin \\ 505 South Hancock Street, Room 552G \\ Louisville, KY 40202 \\ Phone: 502-936-2316 \\ Email: 10jin003@louisville.edu
}

\title{
Present appointment:
}

2016.6-current PhD student in Pharmacology and Toxicology

University of Louisville, Louisville, KY

2009-current Attending doctor at the Department of Anesthesiology

The Second Affiliated Hospital \& Yuying Children's Hospital of Wenzhou Medical College, Zhejiang Province.

P.R.China. 325027

\section{Education}

06/2003

M.D. in Clinical Medicine

Wenzhou Medical University, China

$12 / 2008$

M.S. in Anesthesiology

Wenzhou Medical University, China 
$12 / 2019$

Ph.D. in Pharmacology and Toxicology

University of Louisville, Louisville, KY

\section{Publication}

1. Jin L, Jagatheesan G, Guo L, Nystoriak M, Malovichko M, Lorkiewicz P, Bhatnagar A, Srivastava S, Conklin DJ. Formaldehyde Induces Mesenteric Artery Relaxation via a Sensitive Transient Receptor Potential Ankyrin-1 (TRPA1) and Endothelium-Dependent Mechanism: Potential Role in Postprandial Hyperemia. Front Physiol. 2019 Mar 28;10:277. doi: 10.3389/fphys.2019.00277. eCollection 2019.

2. Jin L, Lipinski A, Conklin DJ. A Simple Method for Normalization of Aortic Contractility. J Vasc Res. 2018;55(3):177-186. doi: 10.1159/000490245. Epub 2018 Jul 5.

3. Jin L, P. Lorkiewicz, M.V. Malovichko, A. Bhatnagar, S. Srivastava, D.J. Conklin*. Acetaldehyde Induces an Endothelium-Dependent Relaxation of Superior Mesenteric Artery: Potential Role in Postprandial Hyperemia. (accepted).

4. L. Jin, G. Jagatheesan, L. Guo, and D.J. Conklin*. Crotonaldehyde-induced Vascular Relaxation: Role of Endothelium and Transient Receptor Potential Ankyrin-1 (TRPA1). (be submited).

5. Jordan Finch, Lexiao Jin (co-author), Andre Richardson, Ganapathy Jagatheesan, Luping Guo, Pawel Lorkiewicz, Zhengzhi Xie, Marina Malovichko, Sanjay Srivastava, Aruni Bhatnagar, Daniel J. Conklin*. Vascular Effects of Inhaled Crotonaldehyde in Mice: Role of TRPA1. (be submitted)

6. Dongyue Zheng, Guowei Wu, Peishun Qin, Bin Ji, Lisha Ye, Tong Shi, Huang, Lexiao Jin $*$. Hyperbaric spinal anesthesia with ropivacaine coadministered with sufentanil for cesarean delivery: a dose-response study. Int J Clin Exp Med 2015;8(4):5739-5745. https://www.ncbi.nlm.nih.gov/pmc/articles/PMC4483882/

7. JIN Le-xiao, QIN Pei-shun, LU Yuan-yuan, et al. The ED50 of gabapentin for postoperative analgesia in patients undergoing gynecological operation. Chinese Journal of Clinical Pharmacy.2016,25 (5):292-295. (Chinese)

8. Jin Lexiao, Dong Chaoxuan, Lu Yuanyuan, et.al. Comparison of Multimodal Analgesic Efficacy of Gabapentin and Ketamine in Hysterectomy. China Pharmaceuticals.2016,25(14):32-35. http://en.cnki.com.cn/Article en/CJFDTotal-YYGZ201614012.htm

(Chinese) 
9. Lexiao Jin, et al. Determining Equivalent Dose of Remifentanil and Fentanyl with Infusing the same Target Concentration of Propofol for Insertion of Laryngeal Mask. China Pharmaceuticals.2009,18(21),7-9. (Chinese) http://www.asaabstracts.com/strands/asaabstracts/abstract.htm\%20\%20;jse $\underline{\text { ssionid=15E5B17FC438839E1E12B4610AD18994 ?year=2010\&index=7\&ab }}$ $\underline{\text { snum }=613}$

10. Lexiao Jin, et al. Determining the ECe50 of propofol for insertion of laryngeal mask in target-controlled infusion combined with fentanyl. Modern Practice Medicine. 2009,12:34-36. (Chinese)

11. Lexiao Jin, et al. Determining the ECe50 of reminfentanil with target concentration of propofol for insertion of laryngeal mask. 2009,25(17),29222925. (Chinese)

\section{Poster and Platform}

1. Poster, 9/2016. "A Simple Method for Normalization of Aorta Contractility." Research! Louisville, Louisville, KY.

2. Poster, 09/2017. Mechanism of Formalin-Induced Vasorelaxation. Research! Louisville, Louisville, KY.

3. Poster, 3/2018. Formaldehyde-induced TRPA1- and EDRF-Dependent Contractile Inhibition in Murine Blood Vessels. SOT annual meeting, San Antonio, Texas.

4. Poster, 09/2018. Diverse Aldehydes Stimulate a Common Endothelium-, Transient Receptor Potential Ankyrin1-, and Nitric Oxide-dependent Pathway in Isolated Murine Blood Vessels. Research! Louisville, Louisville, $\mathrm{KY}$.

5. Presentation, 11/2018. The Cardiovascular Effects of Crotonaldehyde in Vivo and in Vitro. OVSOT student meeting, Louisville, KY.

6. Poster, 3/2019. The Cardiovascular Effects of Crotonaldehyde in Vivo and in Vitro. SOT annual meeting, Baltimore, Maryland.

7. Poster, 09/2019. Vascular Toxicity of Mainstream Cigarette Smoke in Mice: Role of crotonaldehyde and TRPA1. Research! Louisville, Louisville, KY.

8. Poster, 09/2019. Vascular Toxicity of Mainstream Cigarette Smoke in Mice: Role of crotonaldehyde and TRPA1. OVSOT meeting, Cincinnati, $\mathrm{OH}$. 